CHARACTERISTICS AND VARIABILITY OF STORM TRACKS IN THE NORTH

PACIFIC, BERING SEA AND ALASKA

\author{
A \\ DISSERTATION \\ Presented to the Faculty \\ of the University of Alaska Fairbanks \\ in Partial Fulfillment of the Requirements \\ for the Degree of
}

DOCTOR OF PHILOSOPHY

By

Michel dos Santos Mesquita, M.S.

Fairbanks, Alaska

December 2009 
UMI Number: 3401164

All rights reserved

INFORMATION TO ALL USERS

The quality of this reproduction is dependent upon the quality of the copy submitted.

In the unlikely event that the author did not send a complete manuscript and there are missing pages, these will be noted. Also, if material had to be removed, a note will indicate the deletion.

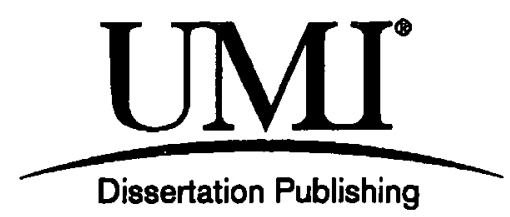

UMI 3401164

Copyright 2010 by ProQuest LLC.

All rights reserved. This edition of the work is protected against unauthorized copying under Title 17, United States Code.

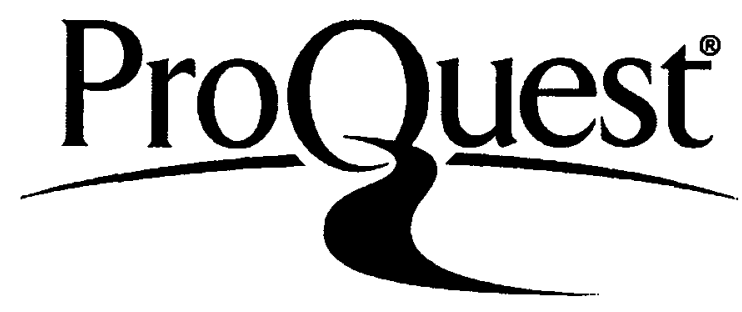

ProQuest LLC

789 East Eisenhower Parkway

P.O. Box 1346

Ann Arbor, Ml 48106-1346 
CHARACTERISTICS AND VARIABILITY OF STORM TRACKS IN THE NORTH

PACIFIC, BERING SEA AND ALASKA

By

Michel dos Santos Mesquita

RECOMMENDED:

APPROVED:

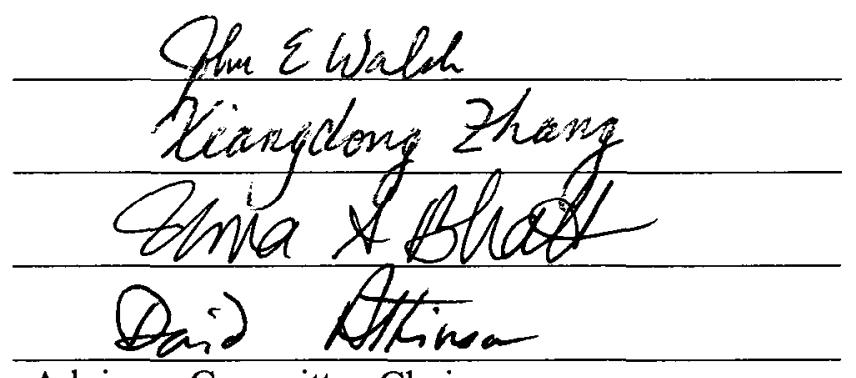

Advisory Committee Chair

rices medeloss

Chair, Department of Atmospheric Sciences
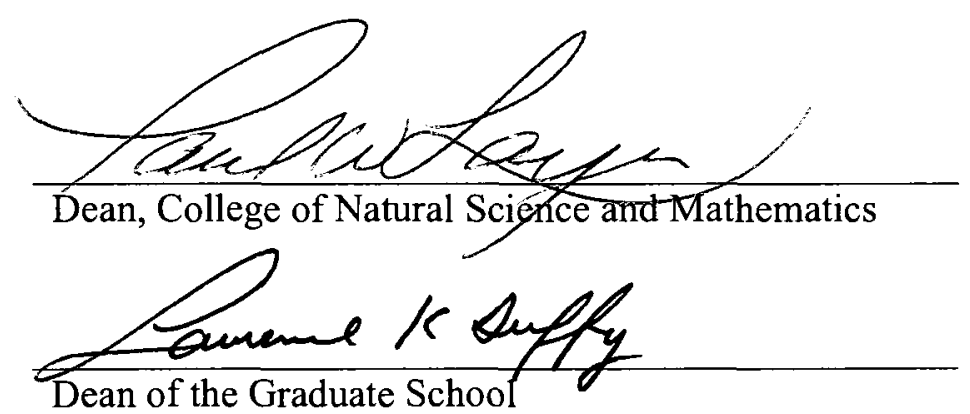

Dean of the Graduate School

Now 30, 2009

Date 


\begin{abstract}
Storm activity in the North Pacific, Bering Sea and Alaska regions is investigated using various automated storm tracking and parameter extraction algorithms. Specific, novel details of storm activity throughout the year are presented. The influence of major climatic drivers is considered, including the Pacific/North American Index and sea ice variability. Details of synoptic-scale forcing on a specific, severe storm event are considered in the context of how different tracking algorithms are able to depict the event.
\end{abstract}

New storm climatology results show that the inter-seasonal variability is not as large during spring and autumn as it is in winter. Most storm variables exhibited a maxima pattern that was oriented along a zonal axis. From season to season this axis underwent a north-south shift and, in some cases, a rotation to the northeast. Barotropic processes have an influence in shaping the downstream end of storm tracks and, together with the blocking influence of the coastal orography of northwest North America, result in high lysis concentrations, effectively making the Gulf of Alaska the "graveyard" of Pacific storms. Summer storms tended to be longest in duration. Temporal trends tended to be weak over the study area. Sea surface temperature did not emerge as a major cyclogenesis control in the Gulf of Alaska.

Positive sea-ice anomalies in the Sea of Okhotsk were found to decrease secondary cyclogenesis, shift cyclolysis locations westward, and alter the North Pacific subtropical jet. In the Atlantic, a negative North-Atlantic-Oscillation-like pattern is 
observed; these results were confirmed by experiments on the ECHAM5 Atmospheric Global Circulation Model driven with sea-ice anomalies in the Sea of Okhotsk.

The destructive west Alaska storm of autumn 1992, which flooded Nome, was investigated using two storm tracking algorithms: NOAA's (National Oceanic and Atmospheric Administration) current operational algorithm and the Melbourne algorithm. Manual tracking was performed as a control. The main storm location features were captured by both algorithms, but differed in the genesis and lysis location. The NOAA algorithm broke the event into two. This storm was shown to have been affected by a blocking high that influenced how the tracking algorithms handled the event. 


\section{Table of Contents}

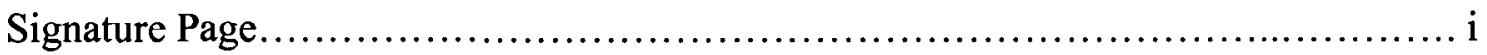

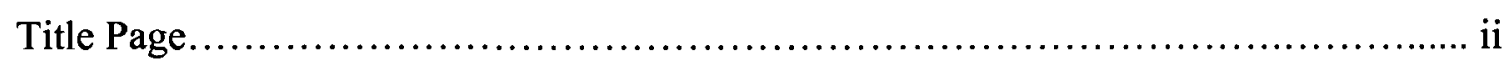

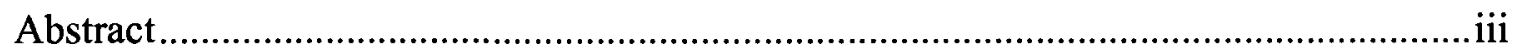

Table of Contents............................................................................................

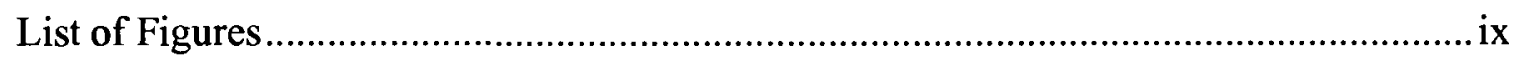

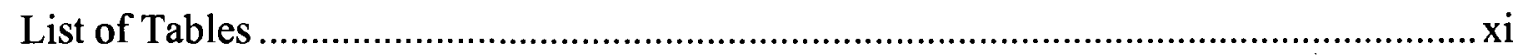

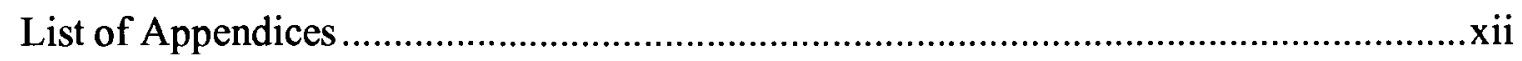

Acknowledgements.......................................................................................

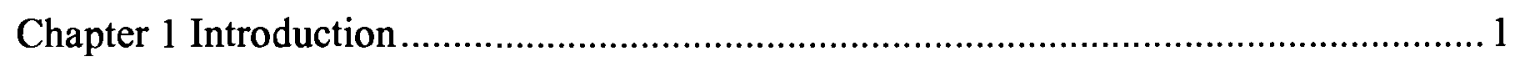

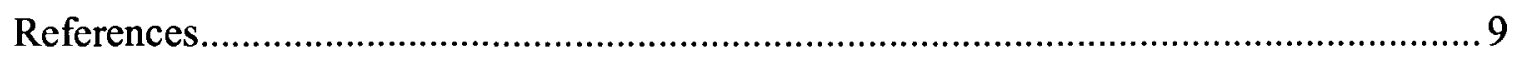

Chapter 2 The "Storm" Concept ........................................................................ 13

2.1. Theoretical Perspective............................................................................ 14

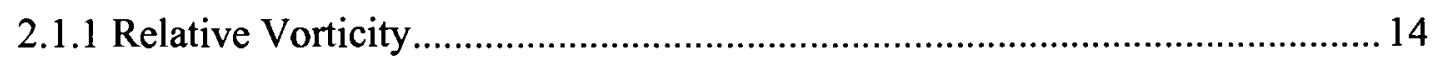

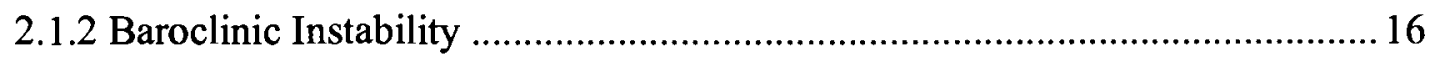

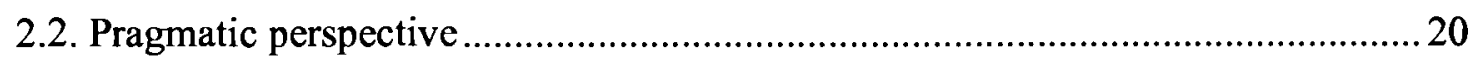

2.3. The Tracking Perspective ........................................................................... 21

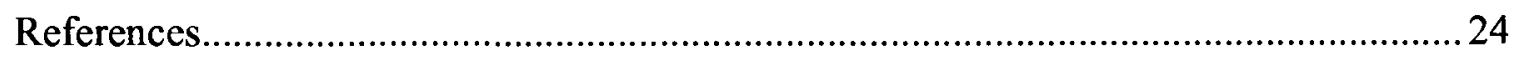

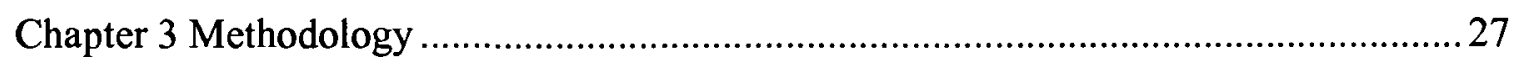




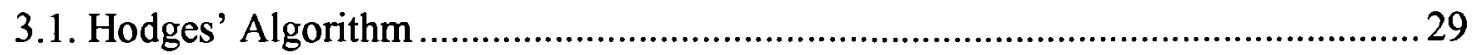

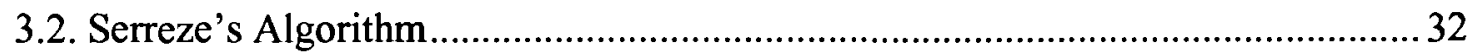

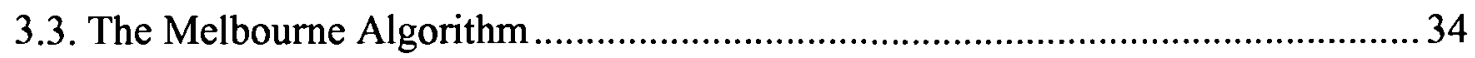

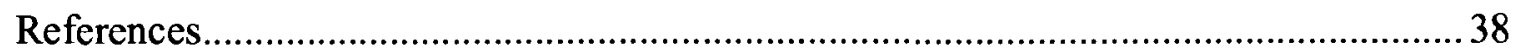

Chapter 4 Characteristics and Variability of Storm Tracks in the North Pacific, Bering

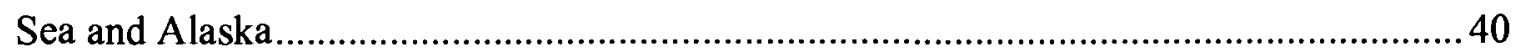

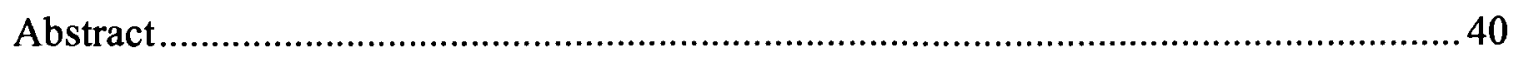

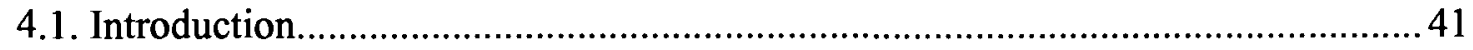

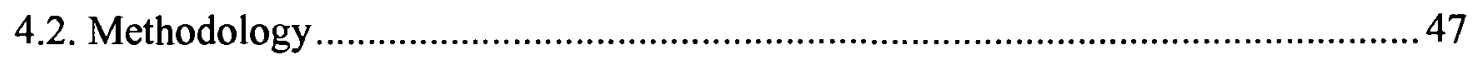

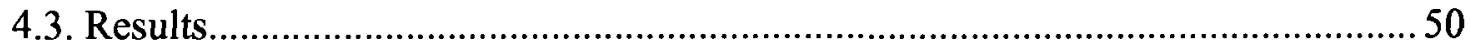

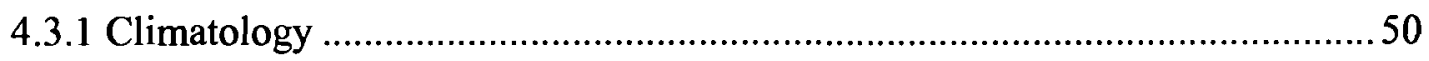

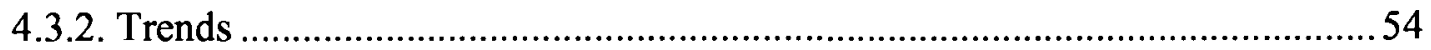

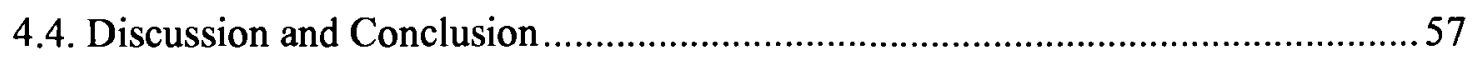

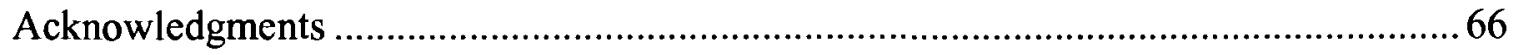

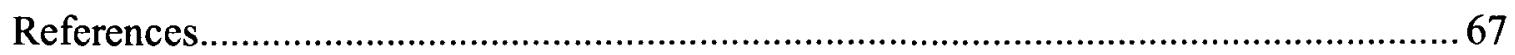

Chapter 5 Sea-ice Anomalies in the Sea of Okhotsk and the Relationship with Storm

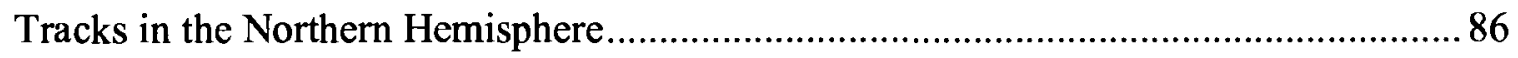

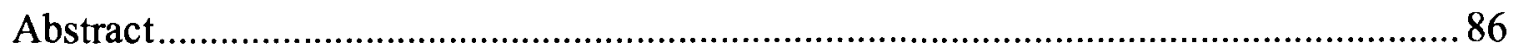

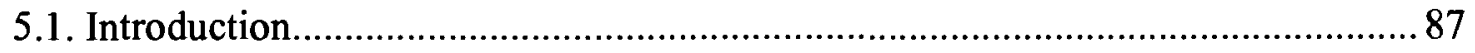

5.2. Methodology and Data Sets .......................................................................... 93

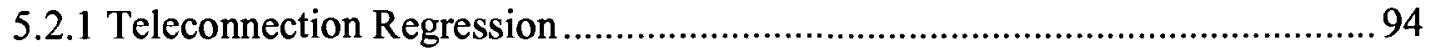

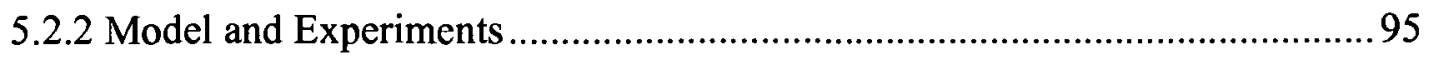


5.2.3 The Compositing Method ........................................................................... 96

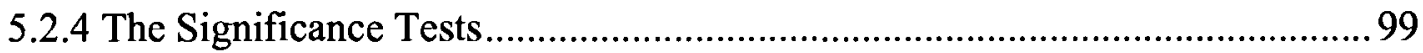

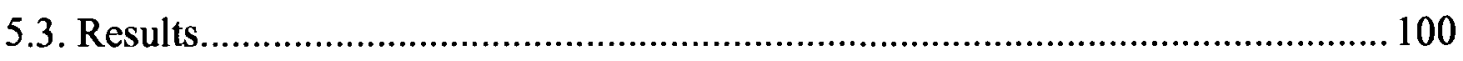

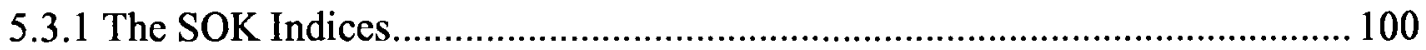

5.3.2 Extra-tropical Storm Tracks and the SOKc Index .................................... 100

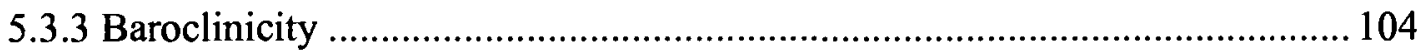

5.3.4 Linear Combination with Teleconnection Patterns ....................................... 106

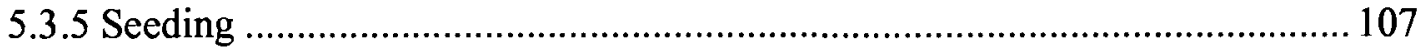

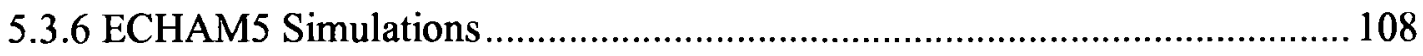

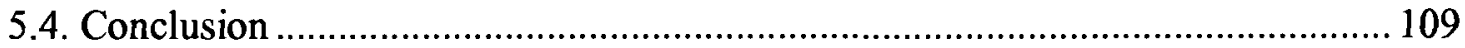

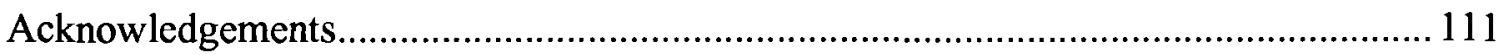

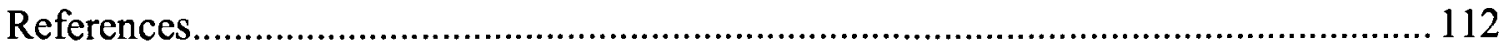

Chapter 6 New perspectives on the synoptic development of the severe October 1992

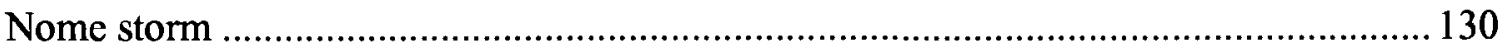

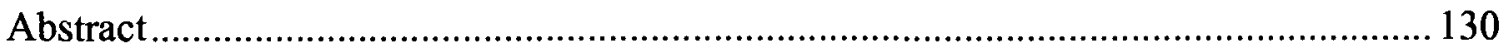

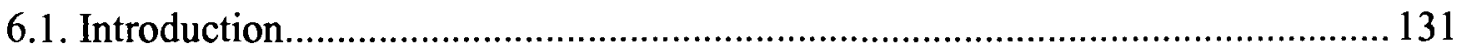

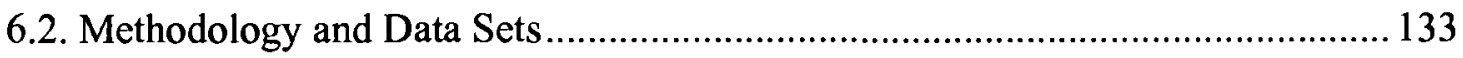

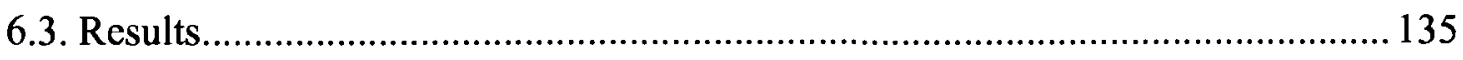

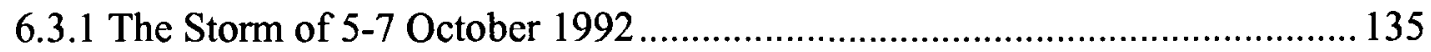

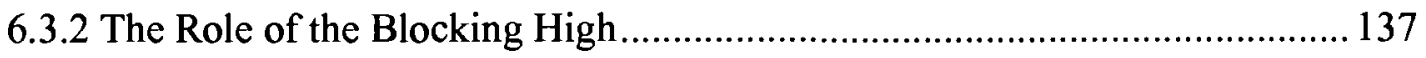

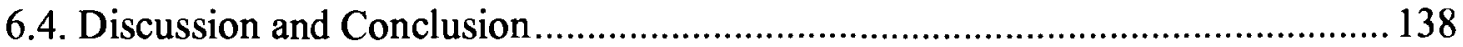

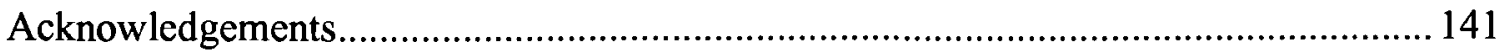




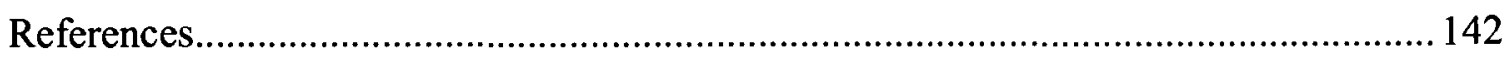

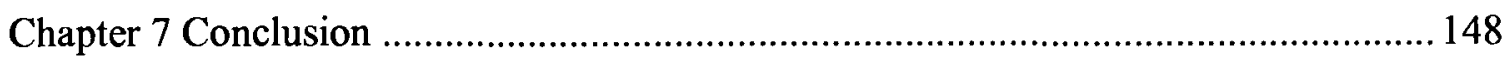

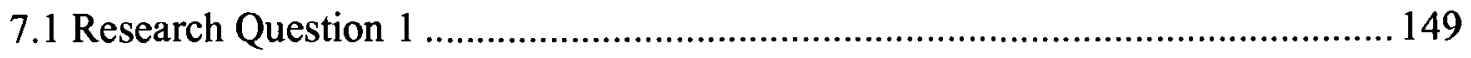

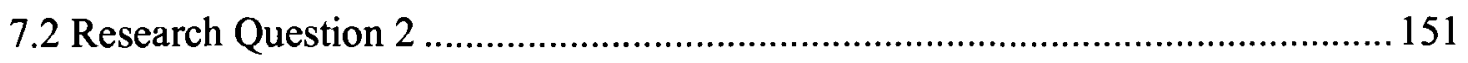

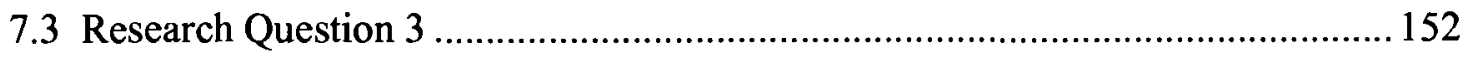

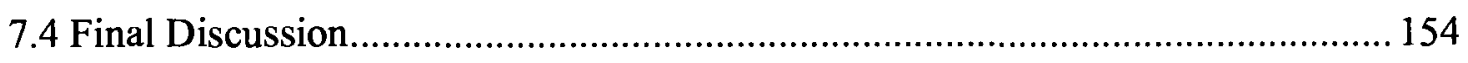

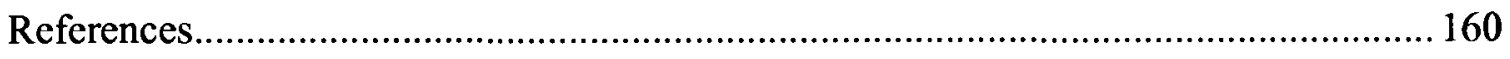

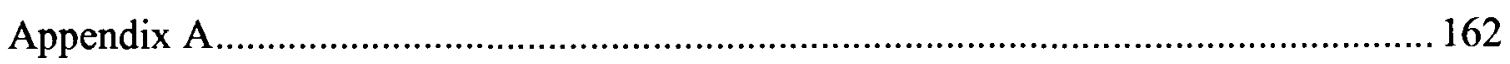

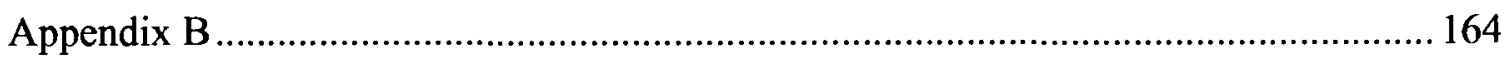

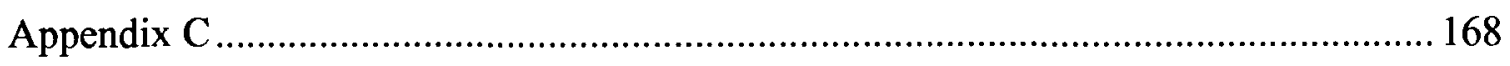

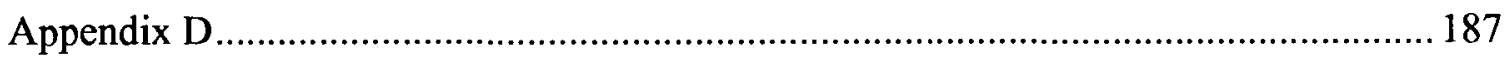




\section{List of Figures}

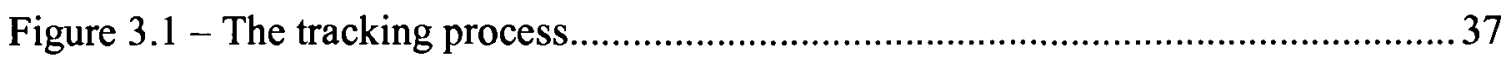

Figure 4.1 - Low over the Aleutian Islands ................................................................ 71

Figure 4.2 - Map of the three regions in Alaska ………............................................. 72

Figure 4.3 - Genesis density climatology ................................................................. 73

Figure 4.4 - Track density climatology ………………............................................. 74

Figure 4.5 - Lysis density climatology ...................................................................... 75

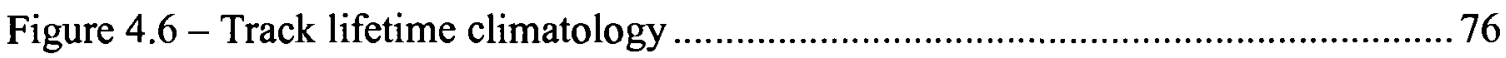

Figure 4.7 - Track intensity climatology .................................................................. 77

Figure 4.8 - Track mean speed climatology ……………............................................. 78

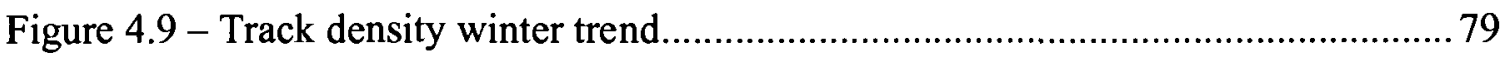

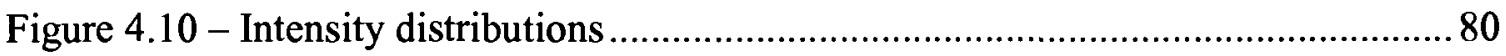

Figure 4.11 - Decadal DJF intensity distributions ………....................................... 81

Figure 4.12 - Mean skin surface temperature in the North Pacific .................................8 82

Figure 4.13 - Genesis density for end-points in GOA ……...................................... 83

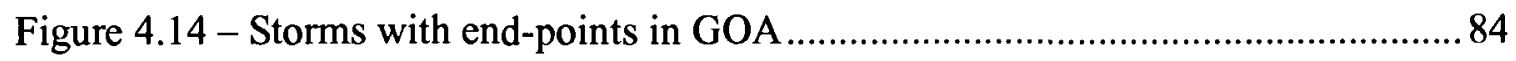

Figure 5.1 - Sea of Okhotsk sea ice concentration...................................................... 117

Figure 5.2 - Epoch analysis showing enhanced positive ice anomalies in SOK ........... 118

Figure 5.3 - Atmospheric circulation anomalies.................................................... 119

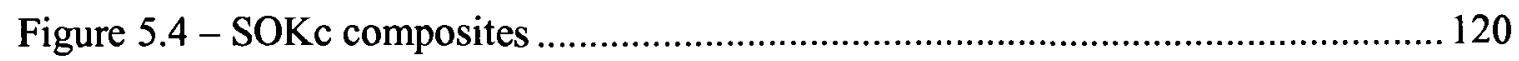


Figure 5.5 - Epoch analysis based on regression equations ..................................... 121

Figure 5.6 - SOKc + minus SOKc- 250hPa composites .............................................. 122

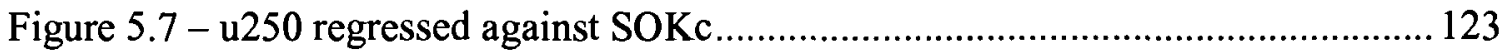

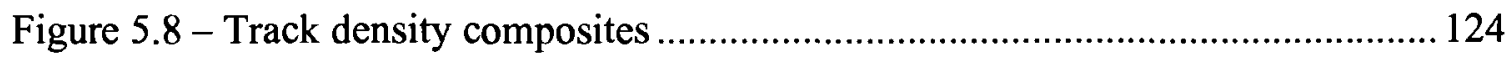

Figure 5.9 - ECHAM5 sea-ice simulation area ..................................................... 125

Figure 5.10 - Sensible plus latent heat flux anomalies ............................................... 126

Figure 5.11 - Geopotential height response from ECHAM5 ……………………........ 127

Figure 5.12 - Epoch analysis from ECHAM5 simulations .......................................... 128

Figure 6.1 - Nome storm MSLP and 500hPa geopotential height plots ....................... 145

Figure 6.2 - Nome storm from three tracking methods ................................................ 146

Figure 6.3 - Life history of the Nome storm ............................................................ 147

Figure C.1 - Synoptic plots of the Nome storm ........................................................ 181

Figure C.2 - Energy and conversion terms time series ............................................. 182

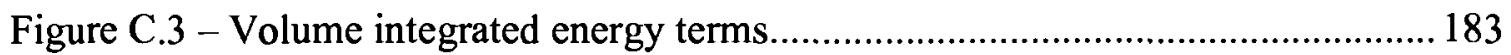




\section{List of Tables}

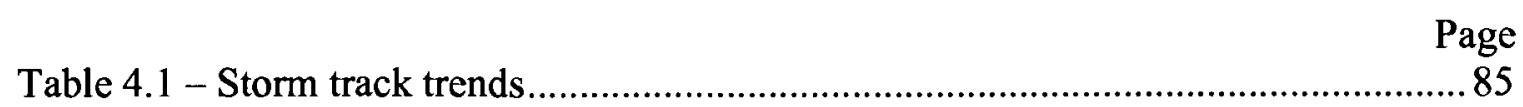

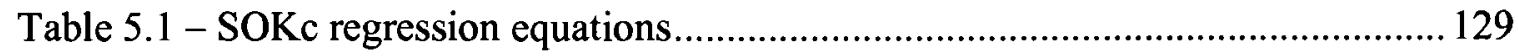

Table 7.1 - Main dissertation results.................................................................. 157

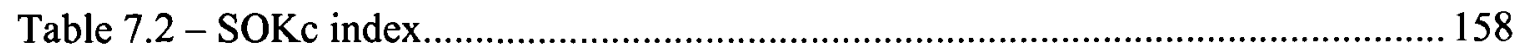

Table 7.3 - Storm parameters from the Melbourne algorithm ................................. 159 


\section{List of Appendices}

\section{Page}

Appendix A - Contributions to the Dissertation Chapters 162

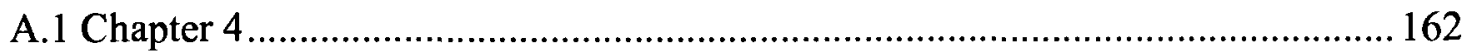

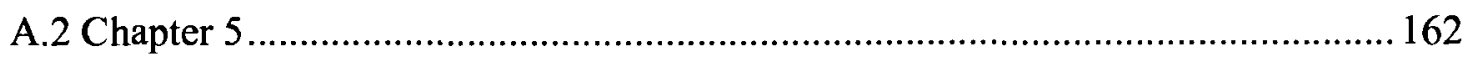

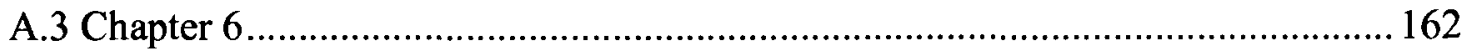

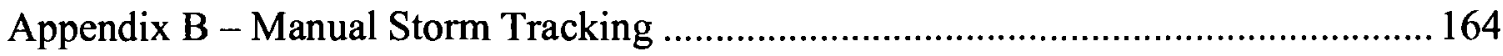

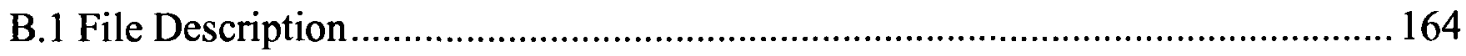

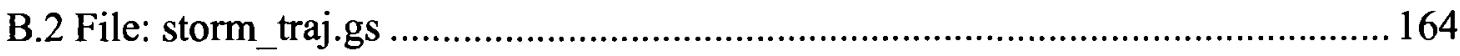

Appendix C - Nome Storm Follow-up: Paper Collaboration ................................. 168

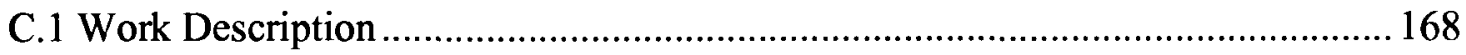

C.2 Environmental Energetics of an Exceptional High-latitude Storm................... 169

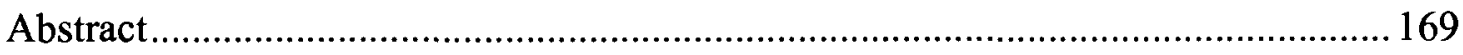

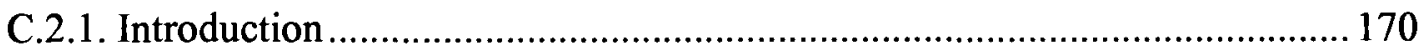

C.2.2. Data and Methodology .............................................................. 172

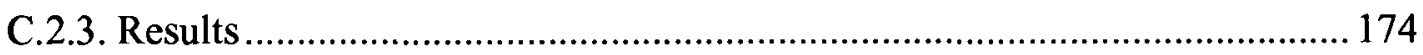

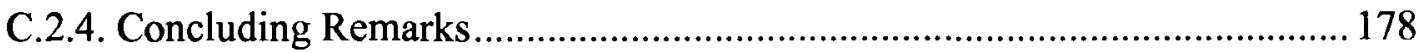

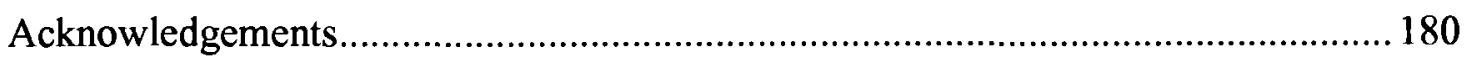

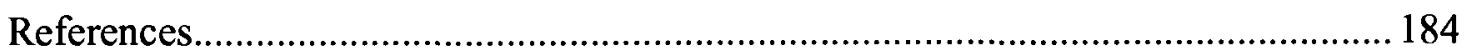

Appendix D - ECHAM5 Usage Justification ............................................ 187

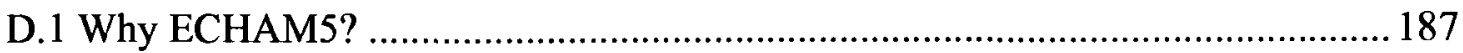


D.2 Global Climate Model Performance over Alaska, Greenland and the Extra-Tropics

D.3 ECHAM5 and the Hodges Algorithm 191

D.4 The Instantaneous Removal of Sea Ice and the Use of Climatological SST ...... 194

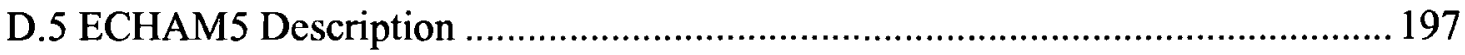

D.5.1 ECHAM5 General Description................................................................ 197

D.5.2 ECHAM5 Sensitivity to Horizontal and Vertical Resolution.........................201

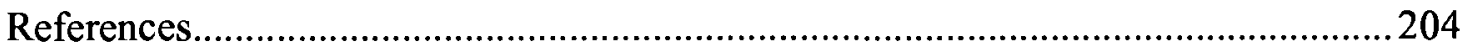




\section{Acknowledgements}

There are many people and institutions that I would like to acknowledge. It becomes almost impossible to list them all here - we come in contact with so many people. I take this opportunity to thank all of those who have been part of my life. I also thank everyone who contributed to this dissertation work, either directly or indirectly. I specially thank Dr. David E. Atkinson, my $\mathrm{PhD}$ advisor, who has given me the opportunity to study at UAF, and who has taught and guided me throughout my studies. I also thank his wife and children for their hospitality. I would also like to thank the committee members, Dr. Uma Bhatt, Dr. John Walsh and Dr. Xiangdong Zhang for the knowledge, guidance, and support given. And I thank the outside examiner, Dr. Amanda Rosenberger, for taking the time to be at the defense. I would also like to express my gratitude to the Interim Dean of the UAF Graduate School, Dr. Lawrence K. Duffy, to the Interim Dean of the College of Natural Science and Mathematics, Dr. Paul Layer, to the IARC Director, Dr. Larry Hinzman, and to the Head of the Atmospheric Sciences Department, Dr. Nicole Mölders.

This dissertation was mainly funded by the National Oceanic and Atmospheric Administration (NOAA) under the project title: "Social Vulnerability to Climate Change in the Alaskan Coastal Zone" (grant NA06OAR4600179). Part of this work was funded by the UAF Center for Global Change \& Arctic System Research and the International Arctic Research Center (IARC) under the 2008 Global Change Student Research Grant 
Competition. My proposal title was: "North Pacific Storm Climatology: Is the Current Operational Algorithm Misrepresenting Alaska Storms?" - I thank Dr. Susan Sugai for the funding provided.

I would like to thank the International Arctic Research Center and the entire staff for the support given. I especially thank Jennifer Moss, Vicky Zins, Kim Cox, and Dr. Elena Sparrow. I also thank Barbara Day (UAF Atmospheric Sciences department) for her great work. I am very grateful for NCAR for providing their data sets in the public domain. And I would also like to thank Mary Haley and Dennis Shea for the support with NCL.

I also take this opportunity to express my gratitude for the support provided by the Bjerknes Centre for Climate Research (Norway) and their researchers, especially Dr. Frode Flatøy, Dr. Nils Gunnar Kvamstø, Dr. Eystein Jansen, Dr. Asgeir Sorteberg, Dr. Idar Barstad, Dr. Helge Drange and Torbjørn Lorentzen. I also thank Bjørn Åge Hjøllo (Jeppesen Marine, Norway) for the support given.

Some researchers have co-authored some of the papers with me, and I would like to thank them very much for that (See Appendix A for the detailed contributions): Dr. Kevin Hodges (University of Reading, UK), Dr. Ian Simmonds (University of Melbourne, Australia), Kevin Keay (University of Melbourne, Australia), Jon Gottschalck (NOAA, USA), and Dr. Juergen Bader (University of Bergen and Bjerknes Centre for Climate Research, Norway). I also thank Dr. Alexandre B. Pezza (University of Melbourne, Australia) for the collaboration work on a follow-up paper of the Nome storm (See Appendix C). 
During my time at UAF/IARC, I was in contact with many professors and researchers. I would like to express my gratitude to them for the teaching and guidance provided: Dr. Javier Fochesatto for the insightful discussions, Dr. Kenneth Sassen for the cloud processes, Dr. Richard Collins for the radiation classes, Dr. William Simpson for teaching chemistry and presentation techniques, Dr. Katrin Iken for the proposal writing classes, Dr. Pia Kohler for the discussions on climate policy, Dr. Margaret Short for the Bayesian probability discussions, Dr. David Newman and Dr. Renate Wackerbauer for the discussions on complex systems, non-linear dynamics and chaos, Dr. Igor Polyakov and Dr. Gleb Panteleev for the ocean discussions, Dr. Vladimir Alekseev for the atmosphere discussions, Dr. Syun-Ichi Akasofu for his research life example, Dr. John Walsh for the inspiration and discussions on some of my manuscripts, Dr. Xiangdong Zhang for the discussions on dynamics, Dr. Uma Bhatt for her charisma, her classes and for the wonderful Friday afternoons in the Climate Journal Club!

I would like to thank previous professors, researchers and educators with whom I had contact with and who have inspired me: Dr. Simone Vieira de Assis and Dr. José Honorato (Federal University at Pelotas, Brazil), Dr. Giovana Ferreira Gonçalves (Federal University at Pelotas, Brazil), Dr. Vilson J. Leffa (Catholic University at Pelotas, Brazil), Dr. Sigbjørn Grønås (University of Bergen, Norway), the memorable Dr. Elmer Raustein (University of Bergen, Norway), Dr. John Michael Wallace (University of Washington, USA), Dr. Ed Carmack (The University of British Columbia, Canada), Dr. Nils Gunnar Kvamstø (University of Bergen, Norway), Dr. Asgeir 
Sorteberg (University of Bergen, Norway), Dr. Joachim Reuder (University of Bergen, Norway), Dr. David Stephenson (University of Exeter, UK), Dr. Kevin Hodges (University of Reading, UK), Dr. Martin King (Monash University, Malaysia), Scott Wales (thelearningxchange.org, USA) and Jandir Venturini (Bells English Center, Brazil).

My colleagues/friends at the Akasofu building (IARC) were a great support. I thank Oceana Francis, Morgan Brown Yarker, Stacy E. Porter, Agatha Light, Stefanie Bourne, Colin Murray, Anna Liljedahl, Brentha Thurairajah, and Dr. Debasish PaiMazumder. I also thank Christopher Swingley who taught me so much about computing and about Alaska. And I thank my friends in Alaska who have helped and supported me in many ways: Judge Victor D. Carlson, Donald H. Crandall, Dr. Martha Shulski, Leandra de Souza, Dr. Sharon Hollensbe, Dr. Megan Brown, Elchin Jafarov, Amy van Hatten, Darla Redger, Amanda Kolker, Sebastien dos Santos, David Podrasky, and Craig Yarker. Another important part of my life at UAF was the contact with the Office of International Programs and the Graduate School: I would like to express my gratitude to Carol Holz, Sue Wolfe and Laura Bender. I also thank the Housing and the Health Departments at UAF for helping make UAF such a great University! And I thank the Golden Key Honour Society UAF chapter (to which I am a member) for welcoming me. 
xviii

I would like to dedicate this dissertation to Frank Tessem (and his family in Norway) and to my family in Brazil: Pedrina dos Santos Mesquita (my mother), Francisco Adir Furtado Mesquita (my father), Simone dos Santos Mesquita (my sister), and Marco Antônio dos Santos Mesquita (my brother). Thank you for the endless phone calls and emails exchanged. I am deeply thankful for being a part of your lives!

Michel dos Santos Mesquita

October 2009

"And the sun also has flashed forth, and the sun has set, and it is coming panting to its place where it is going to flash forth. The wind is going to the south, and it is circling around to the north. Round and round it is continually circling, and right back to its circlings the wind is returning." Ecl. 1:5,6 


\section{Chapter 1 Introduction}

The Arctic is vulnerable to observed and projected changes in climate, according to the Arctic Climate Impact Assessment report (ACIA 2005) and many other Arctic climate projections (Johannessen et al. 2004; Manabe; Stouffer 1994; Serreze et al. 2000). One of the predicted effects of climate change is an increase in severe storm activity (frequency and intensity) in the North Pacific, Bering Sea and Arctic Ocean (Dickson et al. 2000; Graham; Diaz 2001). This correlation between climatic change and storm activity is well-established for the North Pacific from paleoclimatic records (Heusser et al. 1985).

In the Alaska region strong storms are an annual occurrence in the Bering Sea and Gulf of Alaska, with peak activity coming in the fall/winter season (Fathauer 1978). Relatively few of these storms form locally; instead they consist of "old" systems that have moved into the region from the North Pacific and which undergo a process of reenergization - a result of locally favorable patterns in the atmospheric dynamical situation that often occur. Some of these storms stagnate in the southeastern Bering Sea area while others continue north through the Bering Strait and into the Chukchi Sea. These storms represent a serious hazard to users of these marine regions - subsistence and commercial alike - as well as to the many coastal communities. The greatest threats from these storms are waves and water-level surges, a "storm-surge".

The pattern of winds in these systems favors adverse impacts along the west coast of Alaska. The impact of these storms is often significant: powerful winds combined 
with slow-moving systems give time for maximum damaging sea states to develop. Along the coast this means storm surges and heavy wave states. The impact of storm surge on the coastal communities of Alaska can be devastating - many are low-lying and possess vulnerable infrastructure, including airports. For example, Nome suffered the effects of storm surge on October 19, 2004, when a powerful autumn storm moved up from the Aleutian Islands. The storm generated a surge that reached 10.45 feet $(3.20 \mathrm{~m})$, which was able to overtop their seawall, and resulted in overall damages of approximately 20 million US\$. Erosion is another problem faced by Alaska's coastal communities which, being partially caused by wave action, is also related to both the intensity and frequency of storms. Storm-related hazards at the coast are so severe at several locations that the relocation of whole communities is frequently discussed in Alaska. This has severe cultural and economical implications. Other impacts include hazards to Alaska's million-dollar fisheries economy. The primary season for the Bering Sea crab fishery is fall and winter, which presents real dangers for fishing boats. Vessel icing, the accretion of ice on ship decks and structures changing their center of mass and destabilizing the ships, is another hazard especially in the Bering Sea and Gulf of Alaska (Overland 1990). Improving our understanding of the climatology and the dynamics of storms in the Bering Sea, Gulf of Alaska and Alaska is important to better comprehend, prepare for and mitigate coastal erosion and marine hazards.

Research on storms has focused on tracking individual storms to identify typical trajectories, intensities, and possible changes through time, a so-called "Lagrangian" approach. Storm tracking dates back to the mid-nineteenth century when scientists started 
to classify and plot individual storms using synoptic weather maps (Bergeron 1950; Mohn 1870). An important event in meteorology was the first formalized description of the life-cycle of a mid-latitude cyclone in 1919 at the Bergen School, Norway, which made the effective tracking of storms easier. In this early work, storms were tracked by relating their position with time - the Lagrangian approach - and by using statistical methods; e.g., scale variability (Klein 1957; Petterssen 1950). The onset of powerful computer modeling allowed a more global, synoptic approach based on statistical methods through the so-called 'synoptic objective analysis' (Hodges 1995; Hoskins; Hodges 2002; Murray; Simmonds 1991; Serreze et al. 1993; Sinclair 1994; Zhang et al. 2004). This type of analysis made it possible to employ algorithms that identify local minima/maxima of storm diagnostic parameters, such as sea level pressure, within blocks of grid points. This is how most tracking algorithms work today.

For Alaska, the most-used algorithm is that operated by Gottschalck at NOAA's Climate Prediction Center (CPC, now a division of NOAA's Earth System Research Laboratory), which uses the method of Serreze (Serreze 1995; Serreze et al. 1993; Serreze et al. 1997). This algorithm tracks storms by finding the center of a "closed low" surface pressure system, i.e., a grid point is surrounded by points with higher values and which appears as a closed line on a mean sea-level pressure (MSLP) map. Other wellknown algorithms applied in the Northern Hemisphere also use MSLP (Zhang et al. 2004), or by using the Laplacian of the MSLP (Murray; Simmonds 1991) - an alternative approach to finding the field minima. Others use different meteorological variables, such as vorticity (Hodges 1995; Sinclair 1994). 
Storm frequency, geographic locations and life-cycle behavior (e.g. time spent as open vs. closed systems) in the North Pacific are sensitive to changes in the global climate system, as it has been shown in climate simulations of changes in sea-ice and response to the atmosphere and storm tracks (Alexander et al. 2004; Magnusdottir et al. 2004; Mesquita et al. 2008). Mesquita et al. (2008) have also shown that the intensity of summer storms and their lifetime have increased (both at a rate of $5 \%$ per decade) in the North Pacific - a possible response to a warming climate system.

An added uncertainty complicating understanding of storm life-cycle dynamics is the atmosphere-sea-ice interaction. Possible results of this linkage may alter sea-ice configuration (Johnson 1980; Walsh; Johnson 1979b), change the position of storm tracks coming into the North Pacific (Overland; Pease 1982), alter the intensity of storm systems (Murray; Simmonds 1995) and slow down the polar vortex at 500hPa (Murray; Simmonds 1995), among other things. These processes extend beyond the Arctic Ocean and into the Arctic marginal seas; sea-ice plays an important role in the North Pacific as well. For example, the advance of the ice edge in the Bering Sea is based on wind-driven advection, but southerly winds associated with cyclones tracking atypically along the west side of the Bering Sea inhibit this advance (Overland; Pease 1982).

Sea ice acts as a 'lid' reducing the latent and sensible heat fluxes to a cold atmosphere from a relatively warm ocean below, altering the radiation budget significantly (Johnson 1980; Walsh; Johnson 1979a). Some studies have tried to understand the atmosphere-sea-ice relationship using global climate models (GCMs), analyzing how the model reacts to different ice boundary conditions for the entire 
Northern Hemisphere (Murray; Simmonds 1995) and for the North Pacific (Yamamoto et al. 2006). However, there is a need for an updated study relating the changes in sea-ice and the storm track response in the North Pacific. This is mainly the case for the Sea of Okhotsk region, which has been considered in modeling studies (Yamamoto et al. 2006), but not a combination with reanalysis data and a storm tracking algorithm.

Changes in storm tracks and the impact on the coast involve non-linear processes, which need to be understood so that mitigation processes can be more finely tuned. A first step in understanding the problem is to consider the relationship between the largescale flow and storm track systems. If linkages can be demonstrated then changes in the atmospheric flow patterns, such as the El Niño-Southern Oscillation (ENSO), can be used to assess potential storm activity, and by extension, impacts on the coast.

Studies have shown that the atmospheric circulation in the Arctic has changed rapidly (Zhang et al. 2008). A decadal-long shift in the North Pacific atmosphere and ocean after 1976 was also recorded (Trenberth; Hurrell 1994). The shift of 1976 had implications not only for the atmosphere and ocean, but also for the ecosystem-migration of birds and fish - with consequences for Alaska (Mysak 1986; Trenberth; Hurrell 1994). The relationship between storm activity and the large-scale flow has been considered in several papers. A modest association with El Niño was identified in two studies (Graham; Diaz 2001; Key; Chan 1999). These found that cyclogenesis over the Aleutian Islands (at $500 \mathrm{mb}$ ) is reduced during El Niño years compared to La Niña years. A relationship between the NAO and Arctic surface cyclone frequencies in winter and spring was identified in two studies (Key; Chan 1999; Serreze et al. 1997). Ogi et al. 
(2004) identified a connection with summer NAM that was echoed by Mesquita et al. (2008) who further elaborate this point by stating that summer NAM affects the position, but not the strength, of the major baroclinic zones and hence correlates well with storm track density but not intensity.

An array of studies have drawn connections of varying strengths between storm activity and various modes of large-scale variability. A connection with Eastern Atlantic (EA) and Pacific North American (PNA) patterns was identified (Lau 1988; Wallace; Gutzler 1981) as was a shift in the North Atlantic Oscillation (NAO) and increasing storm track activity over Europe (Ulbrich; Christoph 1999); the NAO, the EA, the Scandinavian pattern, the east Atlantic-western Russian pattern, and the polar-Eurasian pattern were used to study the effect of storm track serial clustering (Mailier et al. 2006). Relation to the Arctic Oscillation (AO) (McCabe et al. 2001) and ENSO-like variability (Chang; Fu 2002); AO correlates well with storm intensity over the Atlantic but not over the Pacific (Chang; Fu 2002); the PDO is well correlated with storm track intensity over the Pacific, but not over the Atlantic (Chang; Fu 2002); the Southern Oscillation Index (SOI) is weakly correlated with Pacific storm track intensity, and moderately correlated with the PDO (Chang; Fu 2002); it is not clear to what degree the changes in cyclone activity is related to the PDO (Graham; Diaz 2001). Changes in the storm tracks in the North Pacific help to reinforce and maintain the anomalous circulation in the upper troposphere (Trenberth; Hurrell 1994).

In all the foregoing gaps remain, however. More work is required to provide a more detailed picture of how storms have varied in the North Pacific, Bering Sea, and 
Gulf of Alaska/Alaska regions and how they are linked to specific regionally significant modes of variability. Further work is needed to identify the nature of the influence on storms exerted by sea-ice variability in the Sea of Okhotsk. A closer look is required at how commonly used storm tracking algorithms represent a selected extreme event and the relationship of that event to synoptic drivers. To address these specific topics within the overarching issue of northern hemisphere storm dynamics and variability, the present dissertation is aimed at describing and understanding the characteristics of storm tracks in the North Pacific, Bering Sea, Gulf of Alaska and Alaska regions and their relationship with the large-scale flow. To focus this effort, the research hypothesis of this dissertation is established: Storm activity in the North Pacific, Bering Sea, and Alaska regions has undergone demonstrable change throughout the past decades, activity that is in part attributable to certain features of the physical environment, such as sea-ice variability, and which also can be a function of the tools utilized in the analysis of storms - the tracking algorithms. To test this hypothesis, three specific questions will be answered:

a. What are there characteristics and variability of storm tracks in the North Pacific region and how have they changed?

b. What is the role played by the Sea of Okhotsk sea-ice anomalies on storm activity?

c. How important is the choice of a tracking algorithm and what are some of the synoptic/dynamic influences on a typical strong storm event in the North Pacific? 
Following short introductory/background chapters, the dissertation is divided into three different scientific papers focusing on the three major questions identified above. Specifically, Chapter 2 will discuss the concept of baroclinic instability, the theory behind what a storm represents, how it works dynamically, and how it is linked into the broader atmosphere. Chapter 3 will discuss the methodology of this study, that is, a thorough description of the storm tracking algorithms used. Chapter 4 will develop a climatology of storm tracks in the North Pacific, Bering Sea, Gulf of Alaska and Alaska regions, explaining their characteristics and variability. Chapter 5 will show the connection between sea-ice anomalies in the Sea of Okhotsk and the response in the atmosphere, including implications for storm activity. Chapter 6 will consider a case study of an extreme storm event in the North Pacific/Bering Sea. Chapter 7 will present an overall conclusion. 


\section{References}

ACIA, 2005: Arctic Climate Impact Assessment. Cambridge University Press, 1042 pp.

Alexander, M. A., U. S. Bhatt, J. E. Walsh, M. S. Timlin, J. S. Miller, and J. D. Scott, 2004: The Atmospheric Response to Realistic Arctic Sea Ice Anomalies in an AGCM during Winter. Journal of Climate, 17, 890-905.

Bergeron, T., 1950: De Tropiska Orkanernas Problem. Kungl. Boktr. P. A. Norstedt and Soener.

Chang, E. K. M., and Y. Fu, 2002: Interdecadal Variations in Northern Hemisphere Winter Storm Track Intensity. Journal of Climate, 15, 642-658.

Dickson, R. R., and Coauthors, 2000: The Arctic Ocean Response to the North Atlantic Oscillation. J. Climate, 13, 2671-2696.

Fathauer, T. F., 1978: A forecast procedure for coastal floods in Alaska. NOAA Tech. Memo., NWS AR-23, 27 pp.

Graham, N. E., and H. F. Diaz, 2001: Evidence for Intensification of North Pacific Winter Cyclones since 1948. Bull. Amer. Meteor. Soc., 82, 1869-1893.

Heusser, C. J., L. E. Heusser, and D. M. Peteet, 1985: Late-Quaternary climatic change on the American North Pacific Coast. Nature, 315, 485-487.

Hodges, K. I., 1995: Feature Tracking on the Unit Sphere. Mon. Wea. Rev., 123, 34583465 .

Hoskins, B. J., and K. I. Hodges, 2002: New Perspectives on the Northern Hemisphere Winter Storm Tracks. J. Atmos. Sci., 59, 1041-1061.

Johannessen, O. M., and Coauthors, 2004: Arctic climate change: observed and modelled temperature and sea-ice variability. Tellus $A$, 56, 328-341. 
Johnson, C. M., 1980: Wintertime Arctic Sea Ice Extremes and the Simultaneous Atmospheric Circulation. Monthly Weather Review, 108, 1782-1791.

Key, J. R., and A. C. K. Chan, 1999: Multidecadal Global and Regional Trends in 1000 $\mathrm{mb}$ and $500 \mathrm{mb}$ Cyclone Frequencies. Geophys. Res. Lett., 26, 2053-2056.

Klein, W. H., 1957: Principal tracks and mean frequencies of cyclones and anticyclones in the Northern Hemisphere, Research Paper 40, US Weather Bureau, 60.

Lau, N.-C., 1988: Variability of the Observed Midlatitude Storm Tracks in Relation to Low-Frequency Changes in the Circulation Pattern. J. Atmos. Sci., 45, 2718-2743.

Magnusdottir, G., C. Deser, and R. Saravanan, 2004: The Effects of North Atlantic SST and Sea Ice Anomalies on the Winter Circulation in CCM3. Part I: Main Features and Storm Track Characteristics of the Response. Journal of Climate, 17, 857-876.

Mailier, P. J., D. B. Stephenson, C. A. T. Ferro, and K. I. Hodges, 2006: Serial Clustering of Extratropical Cyclones. Monthly Weather Review, 134, 2224-2240.

Manabe, S., and R. J. Stouffer, 1994: Multiple-century response of a coupled oceanatmosphere model to an increase of atmospheric carbon dioxide. J. Climate, 7, 5-23.

McCabe, G. J., M. P. Clark, and M. C. Serreze, 2001: Trends in Northern Hemisphere Surface Cyclone Frequency and Intensity. J. Climate, 14, 2763-2768.

Mesquita, M. D. S., N. G. Kvamstø, A. Sorteberg, and D. E. Atkinson, 2008: Climatological properties of summertime extra-tropical storm tracks in the Northern Hemisphere. Tellus A, 60, 557-569.

Mohn, H., 1870: Det Norske Meteorologiske Instituts Storm-Atlas. Centen. Proc. Roy. Met. Soc., 17, 26.

Murray, R., and I. Simmonds, 1991: A Numerical Scheme for Tracking Cyclone Centres from Digital Data. Part I: Development and Operation of the Scheme. Aust. Met. Mag., 39, 155-166. 
Murray, R. J., and I. Simmonds, 1995: Responses of climate and cyclones to reductions in Arctic winter sea ice. J. Geophys. Res., 100, 4791-4806.

Mysak, L. A., 1986: El nino, interannual variability and fisheries in the northeast Pacific ocean. Can. J. Fish. Aquat. Sci., 43, 464-497.

Ogi, M., K. Yamazaki, and Y. Tachibana, 2004: The summertime annular mode in the Northern Hemisphere and its linkage to the winter mode. J. Geophys. Res., 109.

Overland, J. E., 1990: Prediction of Vessel Icing for Near-Freezing Sea Temperatures. Weather and Forecasting, 5, 62-77.

Overland, J. E., and C. H. Pease, 1982: Cyclone Climatology of the Bering Sea and Its Relation to Sea Ice Extent. Monthly Weather Review, 110, 5-13.

Petterssen, S., 1950: Some aspects of the general circulation. Centen. Proc. Roy. Met. Soc., 120-155.

Serreze, M. C., 1995: Climatological Aspects of Cyclone Development and Decay in the Arctic. Atmos-Ocean, 33, 1-23.

Serreze, M. C., J. E. Box, R. G. Barry, and J. E. Walsh, 1993: Characteristics of Arctic synoptic activity, 1952-1989. Meteor. Atmos. Phys., 51, 147-164.

Serreze, M. C., F. Carse, R. G. Barry, and J. C. Rogers, 1997: Icelandic Low Cyclone Activity: Climatological Features, Linkages with the NAO, and Relationships with Recent Changes in the Northern Hemisphere Circulation. J. Climate, 10, 453-464.

Serreze, M. C., and Coauthors, 2000: Observational Evidence of Recent Change in the Northern High-Latitude Environment. Climate Change, 46, 159-207.

Sinclair, M. R., 1994: An objective cyclone climatology for the Southern Hemisphere. Mon. Wea. Rev., 122, 2239-2256.

Trenberth, K. E., and J. W. Hurrell, 1994: Decadal atmosphere-ocean variations in the Pacific. Clim. Dyn., 9, 303-319. 
Ulbrich, U., and M. Christoph, 1999: A shift of the NAO and increasing storm track activity over Europe due to anthropogenic greenhouse gas forcing. Climate Dynamics, 15, 551-559.

Wallace, J. M., and D. S. Gutzler, 1981: Teleconnections in the Geopotential Height Field during the Northern Hemisphere Winter. Monthly Weather Review, 109, 784-812.

Walsh, J. E., and C. M. Johnson, 1979a: An analysis of Arctic sea ice fluctuations, 195377. J. Phys. Oceanogr., 9, 580-591.

- , 1979b: Interannual atmospheric variability and associated fluctuations in Arctic sea ice extent. J. Geophys. Res., 84, 6915-6928.

Yamamoto, K., Y. Tachibana, M. Honda, and J. Ukita, 2006: Intra-seasonal relationship between the Northern Hemisphere sea ice variability and the North Atlantic Oscillation. Geophys. Res. Lett., 33, L14711.

Zhang, X., J. E. Walsh, J. Zhang, U. S. Bhatt, and M. Ikeda, 2004: Climatology and Interannual Variability of Arctic Cyclone Activity: 1948-2002. J. Climate, 17, 23002317.

Zhang, X., A. Sorteberg, J. Zhang, R. Gerdes, and J. C. Comiso, 2008: Recent radical shifts of atmospheric circulations and rapid changes in Arctic climate system. Geophys. Res. Lett., 35, doi:10.1029/2008GL035607. 


\section{Chapter 2 The "Storm" Concept}

A precise definition of a "storm" can be somewhat subjective. Depending on what a researcher or user considers a storm to be, the method applied to quantify storm properties can have different results and applications. A storm may be considered under three different perspectives:

a. Theoretical - This has tended to be the most common perspective from which storm track studies have been conducted. In this case, the theory of baroclinic instability is the theoretical framework that helps explain how a storm is formed and evolves; this concept can then feed into other tracking methods, described below, by guiding what variables are used to define a storm, e.g., relative vorticity.

b. Pragmatic - Under this perspective, storms are considered in the context of derivative impacts; for example, for some a storm is defined in terms of how it is manifested at the surface: high winds, clouds, rain, crop damage, and so on. It can also be observed by getting a "snapshot" of mean storm condition, as used in the bandpass filtering methods. Another approach is storm analysis using a Eulerian view of the systems, as used in algorithms based on wind speed at a location, for example.

c. Tracking - Under this perspective an individual storm is discretely identified and monitored over its lifecycle (Lagrangian approach). Analyses of dataset 
generated by several Lagrangian methods form the basis upon which rest the analytical results presented in this dissertation.

These three perspectives are discussed individually below. An overview will be provided in order to set the stage for the "tracking" perspective of this dissertation.

\subsection{Theoretical Perspective}

Cyclogenesis is the term used to describe the development of synoptic-scale weather disturbances. This concept rests upon two essential underpinnings: the presence of thermal imbalance with respect to atmospheric density structure - a baroclinic condition - and the role of relative vorticity centers. While thermal imbalance sets the stage, the presence of local relative vorticity maxima is of particular importance in initiating and maintaining a storm system, and as such is a crucial element for these systems (Holton 2004).

\subsubsection{Relative Vorticity}

Relative vorticity is defined as the vertical component of the vorticity, a measure

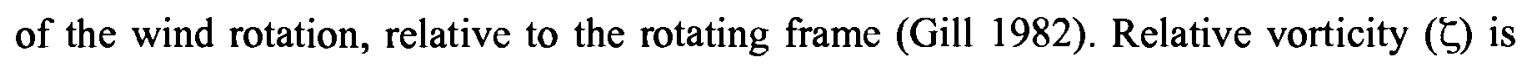
expressed as:

$$
\zeta=\frac{\partial v}{\partial x}-\frac{\partial u}{\partial y}
$$


Where $u$ and $v$ are the zonal and meridional components of the wind, respectively. $x$ and $y$ represent the longitudinal and latitudinal distances, respectively. This equation is derived by calculating the curl of the relative velocity $\omega$ (again, representing a measure of rotation). In the equation below, $\mathbf{u}$ and $\mathbf{v}$ represent the horizontal component of the relative velocity:

$$
\begin{gathered}
\omega \equiv \nabla \times U=\left|\begin{array}{ccc}
\vec{i} & \vec{j} & \vec{k} \\
\frac{\partial}{\partial x} & \frac{\partial}{\partial y} & \frac{\partial}{\partial z} \\
\boldsymbol{u} & \boldsymbol{v} & \boldsymbol{0}
\end{array}\right| \\
\omega=\vec{i}\left(-\frac{\partial v}{\partial z}\right)+\vec{j}\left(\frac{\partial u}{\partial z}\right)+\vec{k}\left(\frac{\partial v}{\partial x}-\frac{\partial u}{\partial y}\right)
\end{gathered}
$$

However, only the vertical component of the relative vorticity is of interest in large-scale meteorology. Expressing the equation for the $\mathrm{k}$ component brings us to the vertical component of interest:

$$
\zeta=\vec{k} \cdot(\nabla \times \boldsymbol{U})=\frac{\partial v}{\partial x}-\frac{\partial u}{\partial y}
$$

Where $k$ represents the vertical unit vector, the nabla operator is applied to the total wind velocity $\boldsymbol{U}$. 


\subsubsection{Baroclinic Instability}

A process that leads to cyclogenesis is dynamical instability, which accounts for the growth of synoptic-scale disturbances (Holton 2004). Due to the thermal wind balance, an intense jet core is found just above this zone of large meridional potential temperature gradients. These jets are unstable with respect to small perturbations. Small disturbances that are introduced into the jet become amplified by drawing additional energy from the jet itself. This is the essential feature of what is termed baroclinic instability (Barry; Carleton 2001). Holton (2004) points out that synoptic disturbances develop "preferentially in the regions of maximum time-mean zonal winds associated with the western Pacific and western Atlantic jets and propagate downstream along storm tracks that approximately follow the jet axes". Here, the author alludes to the other elements of cyclone lifecycle: a baroclinic generation zone, downstream development, and dissipation (Chang; Orlanski 1993).

Baroclinic instability is strongly dependent on the initial conditions. When an upper-level potential vorticity anomaly is advected into a region with a temperature gradient at the surface, this upper-level anomaly will lead to a temperature advection at the surface, inducing a potential vorticity anomaly near the surface. This surface anomaly then reinforces the upper-level anomaly. Both the surface and the upper-level anomaly can become locked in phase with a fast amplification of the anomaly pattern (Holton 2004), or a baroclinic wave. 
The development of the baroclinic wave is also dependent on the ageostrophic (secondary) circulation, otherwise the geostrophic advection would destroy the thermal wind balance (Holton 2004). It is also through the ageostrophic (vertical) circulation that cold advection causes the geopotential height to fall, intensifying the horizontal (northsouth) pressure gradient. It is important to emphasize that the ageostrophic flow is associated with conversion of energy from potential to kinetic energy, another important component of cyclone development (Barry; Carleton 2001).

In order to have conversion of the available potential energy (APE) into kinetic energy, there must be a tilt of the perturbation with height. According to Carlson (1991) APE is defined as the difference between the existing potential energy and that which would result if the temperature field were adiabatically rearranged to become that of the mean state. For this energy conversion to occur, there must be a tilt of the perturbation with height. This implies that the horizontal advection of temperature will increase APE and that the vertical circulation will convert the perturbation APE to perturbation kinetic energy (Holton 2004), necessary for the development of a storm.

The tilt in height occurs when there is a poleward transport of warm air (air rise) and equatorward transport of cold air (air sinking). This process can lower the center of mass of a body of air, allowing the conversion to kinetic energy with time at the expense of a corresponding decrease in potential energy (Carlson 1991). In this fashion, baroclinic instability reduces the pole-to-equator gradient of temperature. A storm can then be considered as a "mixing of air", or a mechanism by which the atmosphere is able to equilibrate temperature gradient anomalies. Storms are thus essential elements of the 
planetary climatic engine, helping to dynamically redress the perpetual state of atmospheric poleward radiative imbalance.

A variety of driving forces can contribute to the maintenance of baroclinic systems: surface heating, convective instability, reduced static stability, moisture availability, latent heat and others. Of these, the release of latent heat is one of the most important driving mechanisms: capable, even, of reinvigorating a dying storm (Grønås et al. 2002). Serreze et al. (1990) point out that surface heat fluxes associated with future cryospheric states that feature more open water and greater flaw-leads in sea-ice, in part due to cyclone activity, may provide a feedback to help maintain a cyclone. The latent heat released during the rising air over the warm sector of cyclones acts as an additional energy source to the eddies within the storm tracks, whereas the surface sensible heat fluxes (mainly over the ocean), act as an energy sink, reducing the temperature perturbations close to the surface (Branscome et al. 1989).

The weather systems of the mid-latitudes are organized geographically: they have distinct zones of typical occurrence, moving along "preferred" pathways termed "storm tracks". Storm tracks are strongly associated with zones of baroclinic generation as described above. Hoskins and Valdes (1990) proposed a threefold process which allows for an enhancement of baroclinicity over the storm track entrance regions in the Northern Hemisphere:

a. Storm track eddies are vigorous downstream of maximum baroclinicity, and the mixing of temperature by eddies is better where baroclinicity is largest. 
The mixing process is important for the development and decay of the storm;

b. Storm tracks are self-maintained, since diabatic heating maxima are caused by the eddies themselves (condensational heat over the storm track entrance region). It must be noted that this is questioned by Lee and Mak (1996), who argue that enhanced baroclinicity over the storm track entrance region could be maintained by stationary waves induced by mountains alone, without the need for diabatic heat sources near the storm track entrance regions;

c. Warm, western ocean currents (Kuroshio, Gulf stream) are driven by the wind stress of the low-level flow induced by the eddies, which establish zones of high baroclinicity due to land-sea temperature contrasts.

Chang and Orlanski (1993) consider storm tracks transients as an ensemble of wave packets (with wave growth and decay) happening over all portions of the storm track. The individual synoptic eddies within the nonlinear wave packets decay by transferring their energy to their neighbor eddies downstream (downstream development). 


\subsection{Pragmatic perspective}

A "storm" for an observer can be considered as his/her perception of the system. For example, when it gets windy and rainy someone might say that this is a "storm". This perception concept can be found even in ancient documents and literature that detail "stormy" events. Although these represent qualitative accounts of events, the perception factor can be of great importance when studying extreme events in the absence of measured data. For example, both written and word-of-mouth accounts and a person's experience can be taken into account for formulating extreme-value statistics using a Bayesian approach. According to Sivia and Skilling (2006), the Bayesian framework contends that a probability represents how much we believe that something is true. To be useful, though, a belief should be based on all the relevant information available. Laplace found one of the most important discoveries in celestial mechanics by using such probability approach to estimate the mass of Saturn, given orbital data from various astronomical observatories (Jaynes 2003; Sivia; Skilling 2006). The strength of this approach is evident, as Laplace's original estimate has changed by only $0.63 \%$ down to the present day (Sivia; Skilling 2006).

Methods have also been developed to quantify this "storm" perception. This can be done by considering a Eulerian perspective and using atmospheric variables and thresholds to define when there is a storm event. Atkinson (2005) developed an algorithm that counts storm events based on winds. His results are pragmatic and very useful for considering the effect a storm can have. A storm is not just the center of a cyclone system, but it is also the effect observed at a distance within its radius of 
influence. Atkinson's scheme is one of the only algorithms that measures this awayfrom-the-center effect, and it can be applied for mitigation studies as well.

The bandpass filtering (Blackmon 1976; Blackmon et al. 1977), which provides a "snapshot" of the storm track location may also be classified under a "pragmatic" perspective. Exact counts of storm systems are not given, but the main track location can be identified. Bandpass filtering diagnoses storm track activity by determining statistics associated with pre-defined synoptic timescales, for example, from between 2 to 10 days for a mid-latitude storm. Barry and Carleton (2001) indicate that the results of bandpass filtering depict a zone resembling that of storm tracks over the oceans. Although they highlight spatial patterns associated with various timescales, these patterns are not always related to storms (Anderson et al. 2003). A narrow band only emphasizes a particular timescale when cyclones may have different timescales associated with their lifecycles (Mesquita et al. 2008).

\subsection{The Tracking Perspective}

Storms can be studied by tracking each individual storm system and following it in time - the general term for this method is a Lagrangian approach. Even from this perspective, however, the concept of what a storm is must still be considered: different definitions can result in different outcomes. This is manifested when determining which atmospheric variable should be used to categorize a storm, or similarly the question of which level to use. Clearly there are numerous possible combinations. In this sense, 
direct comparisons of different tracking algorithms can be difficult to the point of uselessness.

The tracking approach can be sub-divided along two procedural lines: manual and automated detection. Manual tracking has been used in a number of studies (Keegan 1958; Lydolph 1977; Pickart et al. 2008; Pickart et al. 2009; Whittaker; Horn 1984), it is limited in scope - automated methods generate far more results - but valuable in detail and fidelity - results are accurate and to the point of the study. With the advent of more powerful computers and model-generated grids in the last 20 years, "automated" or "objective" algorithms have emerged and been used to identify storm systems (Hodges 1995; Murray; Simmonds 1991; Serreze et al. 1993; Sinclair 1994; Zhang et al. 2004).

The main focus of this dissertation is on "feature" tracking, where discrete cyclones are identified and tracked in time. A brief description of "feature" tracking is described briefly below and in greater detail in Chapter 3 and in the relevant sections in the major dissertation chapters.

Several variables and altitude levels have been used to study storm tracks. Hoskins and Hodges (2002) point out that the $250 \mathrm{hPa}$ relative vorticity and $330 \mathrm{~K}$ potential vorticity levels are useful for studying upper atmospheric levels. They indicate that using the $850 \mathrm{hPa}$ level is appropriate for storms work because the positive meridional wind can be associated with the warm sectors of storms and negative meridional wind with the cold air behind the systems at this level. In general, most tracking algorithms use the mean sea level pressure (MSLP) to track storms either 
directly (Serreze 1995; Zhang et al. 2004), or using the Laplacian of the MSLP (Murray; Simmonds 1991).

In this dissertation we follow the ideas presented in Mesquita et al. (2008) page 558: "thinking in absolute terms of trying to pick a 'best' method is not profitable because the act of precisely defining a 'storm' can possess a certain degree of subjectivity, rendering logical bases for justification somewhat circular" - and so do not limit use of algorithms in any absolute sense. 


\section{References}

Anderson, D., K. I. Hodges, and B. J. Hoskins, 2003: Sensitivity of feature-based analysis methods of storm tracks to the form of background field removal. Mon. Wea. Rev., 131, 565-573.

Atkinson, D. E., 2005: Observed Storminess Patterns and Trends in the Circum-Arctic Coastal Regime. Geo-Mar. Lett., 25 (2-3), 98-109.

Barry, R. G., and A. M. Carleton, 2001: Synoptic and Dynamic Climatology. Routledge, $620 \mathrm{pp}$.

Blackmon, M. L., 1976: A climatological spectral study of the $500 \mathrm{mb}$ geopotential height of the Northern Hemisphere. J. Atmos. Sci., 33, 1607-1623.

Blackmon, M. L., J. M. Wallace, N. C. Lau, and S. J. Mullen, 1977: An observational study of the northern hemisphere wintertime circulation. J. Atmos. Sci., 34, 1040-1053.

Branscome, L. E., W. J. Gutowski, and D. A. Stewart, 1989: Effect of Surface Fluxes on the Nonlinear Development of Baroclinic Waves. Journal of the Atmospheric Sciences, 46, 460-475.

Carlson, T., 1991: Mid-latitude weather systems. Routledge.

Chang, E. K. M., and I. Orlanski, 1993: On the Dynamics of a Storm Track. Journal of the Atmospheric Sciences, 50, 999-1015.

Gill, A. E., 1982: Atmosphere-ocean dynamics. Vol. 30, Academic Press.

Grønås, S., N. G. Kvamstø, and E. Raustein, 2002: Numerical simulation of the northern Germany storm of 27-28 August 1989. Tellus $A, \mathbf{4 6}, 635-650$.

Hodges, K. I., 1995: Feature Tracking on the Unit Sphere. Mon. Wea. Rev., 123, 34583465. 
Holton, J. R., 2004: An Introduction to Dynamic Meteorology. 4 ed. Elsevier Academic Press.

Hoskins, B. J., and P. J. Valdes, 1990: On the Existence of Storm-Tracks. J. Atmos. Sci., 47, 1854-1864.

Hoskins, B. J., and K. I. Hodges, 2002: New Perspectives on the Northern Hemisphere Winter Storm Tracks. J. Atmos. Sci., 59, 1041-1061.

Jaynes, E. T., 2003: Probability theory: the logic of science. Cambridge University Press.

Keegan, T. J., 1958: Arctic Synoptic Activity in Winter. J. Atmos. Sci., 15, 513-521.

Lee, W.-J., and M. Mak, 1996: The Role of Orography in the Dynamics of Storm Tracks. Journal of the Atmospheric Sciences, 53, 1737-1750.

Lydolph, P. E., 1977: Climates of the Soviet Union. Vol. 7, Elsevier Scientific Publishing Company.

Mesquita, M. D. S., N. G. Kvamstø, A. Sorteberg, and D. E. Atkinson, 2008: Climatological properties of summertime extra-tropical storm tracks in the Northern Hemisphere. Tellus $A, 60$.

Murray, R., and I. Simmonds, 1991: A Numerical Scheme for Tracking Cyclone Centres from Digital Data. Part I: Development and Operation of the Scheme. Aust. Met. Mag., 39, 155-166.

Pickart, R. S., G. W. K. Moore, A. M. Macdonald, I. A. Renfrew, J. E. Walsh, and W. S. Kessler, 2008: Seasonal evolution of Aleutian low-pressure systems: Implications for the North Pacific sub-polar circulation. J. Phys. Oceanogr., 39, 1316-1339.

Pickart, R. S., G. W. K. Moore, D. J. Torres, P. S. Fratantoni, R. A. Goldsmith, and J. Yang, 2009: Upwelling on the continental slope of the Alaskan Beaufort Sea: Storms, ice, and oceanographic response. J. Geophys. Res., 114. 
Serreze, M. C., 1995: Climatological Aspects of Cyclone Development and Decay in the Arctic. Atmos-Ocean, 33, 1-23.

Serreze, M. C., J. A. Maslanik, R. H. Preller, and R. G. Barry, 1990: Sea ice concentrations in the Canada Basin during 1988: Comparisons with other years and evidence of multiple forcing mechanisms. J. Geophys. Res., 95, 22253-22267.

Serreze, M. C., J. E. Box, R. G. Barry, and J. E. Walsh, 1993: Characteristics of Arctic synoptic activity, 1952-1989. Meteor. Atmos. Phys., 51, 147-164.

Sinclair, M. R., 1994: An objective cyclone climatology for the Southern Hemisphere. Mon. Wea. Rev., 122, 2239-2256.

Sivia, D. S., and J. Skilling, 2006: Data analysis: a bayesian tutorial 2ed. Oxford University Press.

Whittaker, L., and L. Horn, 1984: Northern Hemisphere Extratropical Cyclone Activity for Four Mid-season Months. J. Climatol., 4, 297 - 310.

Zhang, X., J. E. Walsh, J. Zhang, U. S. Bhatt, and M. Ikeda, 2004: Climatology and Interannual Variability of Arctic Cyclone Activity: 1948-2002. J. Climate, 17, 23002317. 


\section{Chapter 3 Methodology}

This chapter provides an overview of the three tracking algorithms used in the dissertation. They form the main core of the methodology applied during the research work. Other details about the dataset used and specific information about how the algorithms were applied are discussed in the Methodology section of each of the three papers.

The storm tracking procedure is normally applied to a projected, gridded dataset. Tracking a storm may be described as a three-way process (See Fig. 3.1).

In the first instance (Fig. 3.1A), feature points are identified for each time step (from timestep 1 to $\mathrm{n}$ ). These points can be a pressure minima (Serreze 1995; Zhang et al. 2004), the maximum Laplacian of a pressure field (Murray; Simmonds 1991), or points which correspond to the threshold of a field (Hodges 1995). In Fig. 3.1A these points are represented as $1 s$, as opposed to the $0 s$, meaning that there is no feature point in that grid point.

The second step (Fig. 3.1B) represents the linkage between the feature points in successive time steps to form a storm track. This is a delicate process, errors in which can compromise the results. For example, in Fig. 3.1B, the gray box represents a point from timestep 3 which could be considered as the continuation of the storm being tracked. However, in the illustration, the algorithm chose another feature point. There are different techniques to deal with how feature points can be connected. The most prominent (or common) ones are nearest neighbor, which consists of finding feature 
points closely located in the posterior timesteps (Serreze 1995), using probability based on climatology to determine the possible location of the feature point and then choosing the 'best' point (Murray; Simmonds 1991), or using a cost function (Hodges 1999).

The third step (Fig. 3.1c) is the actual calculation of the storm track statistics using a database of events resulting from the first two steps in Figure 3.1. These are determined by counting the number of occurrences of cyclones in boxes (latitude/longitude) and determining the frequency (number of events in a box divided by the number of timesteps), the density (number of centers per unit area) or the number of features which cross a box (frequency/density/flux), as described in Hodges (1996).

Since most of the studies have been applied to tracking on some projection, the frequency distributions are often normalized to correct for the projection distortion. But such corrections can introduce biases (Hodges 1996). Some algorithms also have constraints related to when to start counting a system as a 'storm', for example, they can use a lifespan threshold stating that a storm must last more than 2 days before counting is performed. Cyclogenesis (where storms start) and cyclolysis (where they die) can be determined by considering the first/second and penultimate/last feature point of a storm track.

Next, a brief overview of the three algorithms is provided. 


\subsection{Hodges' Algorithm}

For Hodges (1996), the term "cyclonic" (closed low) is not necessarily synonymous with vorticity centers; instead, the term "cyclonic activity" is used since a cyclonic vorticity center is a center of cyclonic activity. Hodges' algorithm is called TRACK and it uses a series of steps for the tracking. Here, a brief description of the algorithm is provided, more details may be obtained in Hodges (1995).

The first step is the selection, where the user can select a region and a threshold value. Then the segmentation process divides the selected region into distinct areas and it gives all points (or pixels) a unique label; the identification step looks for points that have intensities larger than the user-defined threshold value (extrema in the vorticity, for example). The ones which have a value that is less than the specified threshold are called 'background' points, as shown in Figure 3.1 and represented by the equation below:

$$
b_{i, j}= \begin{cases}1, & f_{i, j} \geq T_{i}=N_{x}, \ldots, N_{x}+2^{n}-1 \\ 0, & f_{i, j}<T_{j}=N_{y}, \ldots, N_{y}+2^{m}-1\end{cases}
$$

Where $b_{i, j}$ is the binary field value, and $f_{i, j}$ is the actual field values. $\mathrm{T}$ is the value of the threshold, and $N x$ and $N y$ represent a corner node of the region of interest (Hodges 1994).

The matrix process consists of the creation of the binary maps, which are then converted to hierarchy levels called "data hierarchy"; Boundary points is the process that takes care of identifying feature points that are leaving and entering the region of interest. 
These go through a filtering process and new labeling; The feature detection step identifies suitable points within the binary map for tracking the storms; for example, centroids or local extrema maxima within each object, by comparing each object point with its neighbours. If clusters of object points are found, their centroid is detected and the cluster is represented by a single feature point. The position of the feature point and the point value are recorded. The last step is the correspondence, which links the points from the first to the second frame and so on. In order to solve the correspondance problem between the feature points in consecutive frames, a cost function is applied (Hodges 1999).

TRACK uses a threshold to remove tracks that last less than 2 days or which travel less than 5 degrees along a great circle arc (about $500 \mathrm{~km}$ ) (Hodges 1999). A cost function is used based on measures of local track smoothness (measured in terms of changes in direction and speed). A number of three consecutive frames are required for the ensemble statistics. First, it uses the different frames to locate and index each storm center found. After that, if incomplete tracks are found, they are padded out with "phantom" feature points, so that all tracks have the same number of points and span the length of the timeseries. Then the optimization swaps points on the tracks to give the greatest gain in smoothness and it goes on both forward and backward in time to guarantee that there is no point ordering dependence in the final result (Hodges 1999).

Storms are tracked in the unit sphere, which prevents the introduction of latitudinal biases into the tracking (Hodges 1995). For the statistical calculation, kernel estimators are created on the sphere. Working directly on the sphere reduces the introduction of 
systematic biases and the need for latitude-dependent normalization/corrections are irrelevant (Hodges 1996).

The storm parameter outputs generated by this function include:

- genesis density, which shows areas where the largest densities of cyclogenesis are (where systems start);

- track density, the number of storm tracks through a region per season. It uses a single point from each track that is closest to the estimation point which ensures a low pressure system is counted just once for each area studied. It is the number of low pressure tracks and not the low pressure centers;

- feature density, the density (number per unit area) of low pressure centers. Slow moving systems will contribute more to the density in a small region;

- lysis density, the regions where a low pressure system dies (cyclolysis);

- mean track intensity, computed from the depth of the feature points measured in vorticity units;

- mean track speed, the mean speed of a storm track;

- mean lifetime, the mean lifetime (days) of tracks passing through some region. 


\subsection{Serreze's Algorithm}

Serreze's algorithm is most prominent in the United States, especially at the National Oceanic and Atmospheric Administration. It is a practical and fast algorithm which has been used in many studies (Serreze 1995; Serreze et al. 1993). It also closely emulates the manual tracking technique in the sense that it tracks storms by finding the center of a "closed low" pressure system, i.e., a grid point is surrounded by points with higher values and appears as a closed line on a mean sea level pressure map. A userdefined threshold is used as the minimum difference between the central point and the surrounding ones on a $3 \times 3$ grid. A choice of threshold below $2 \mathrm{mb}$ has proven most suitable (Eichler; Higgins 2006). Points within the threshold are identified as a cyclone center (Serreze et al. 1993). If the threshold condition is not fulfilled and none of the eight pressure values fall from the tested central point, the next surrounding "shell" of 16 points is considered (Serreze 1995; Serreze et al. 1993). If in each direction from the central point (adjacent or the next outer grid point) the pressure increases by at least the threshold value, the center point passes as a cyclone center. If the pressure increases by less than the threshold value, the third surrounding shell is tested. Systems above a certain pressure-value threshold are discarded (e.g.: $1012 \mathrm{mb}$ in Serreze et al. (1993)). Duplicates are removed by averaging. Up to three grid points away from the central point are tested, giving a maximum detection diameter of about $2400 \mathrm{~km}$ (Serreze 1995).

In order to link the cyclone centers, a grid array is centered over the first timestep. If a cyclone falls within the array, the chart 2 cyclone at the center of the array is taken to be a continuation of the chart 1 system (Serreze et al. 1997). In Serreze et al. (1997), a 
3x3 National Meteorological Center (NMC) grid was used as the grid array size (NMC at a $47 \times 51$ octagonal grid). The authors mention that two or more chart 1 systems within the same $3 \times 3$ grid is rarely observed. Normally, two or more untracked chart 2 systems have a minimum distance with respect to the same chart 1 system, and here the number of the chart 1 system is carried over to the closest chart 2 system (Serreze et al. 1997). This happens only when the candidate chart 2 system moves less than $1400 \mathrm{~km}$, moves less than 10 degrees south and exhibits sea level pressure tendency with an absolute value less than $40 \mathrm{mb}\left(12 \mathrm{~h}^{-1}\right)$, as pointed out by Serreze (1995). If these conditions are not satisfied, the chart 2 system is taken as new (cyclogenesis), and further chart 2 systems are tested (up to the $1400 \mathrm{~km}$ limit) (Serreze 1995). All remaining chart 1 systems that were not paired with a chart 2 system are considered to have filled (cyclolysis). The linking process is repeated for the subsequent timesteps and the storm tracks are assigned numbers.

The algorithm output includes, among other things, cyclone position and central pressure (Serreze et al. 1997). For the statistics computation, a Cartesian grid is used where the latitude and longitude value of the storm centers are converted to $x$ and $y$ values. Grid cells are $5 \times 5$ degree boxes which do not vary by latitude and which form an area of $309,000 \mathrm{~km}^{2}$ (Serreze et al. 1993). Systems are counted in each grid cell and converted into percent frequencies. As for the Arctic, Serreze et al. (1993) considers the equal-area box approach better than applying area normalization to raw counts, which could introduce biases. Only systems lasting two days or longer are used in the statistics. 


\subsection{The Melbourne Algorithm}

The Melbourne algorithm analytically models the pressure field between grid points. It fits the field using a bicubic spline to the entire array on a polar stereographic grid. Pressures are then interpolated by applying a Taylor's expansion to the pressure field out to the $15^{\text {th }}$ derivative of the field variable (Murray; Simmonds 1991). An instantaneous pressure field is thus thought of as the superposition of bell-shaped functions of different amplitudes upon a climatic average field (background field) (Murray; Simmonds 1991). When considered in this manner, a storm can be counted earlier in its lifetime, that is, even before becoming a "closed" system on a map. This allows better identification of source regions (Hodges 1996). Murray and Simmonds (1991) also contend that "...a higher order fit ... is a better approximation than the essentially linear representation of a grid-based method".

In order to avoid a "checkerboard" type of motion when the grid spacing is too large, the track finding procedure consists of two separate processes (Murray; Simmonds 1991). An initial scanning procedure seeks grid-points at which the Laplacian of the pressure is greater than at any of the eight surrounding points and greater than a prescribed positive value. This method is less restrictive than just comparing values at neighboring points. The Laplacian of the pressure is given as:

$$
\nabla^{2} p\left(x_{i}, y_{i}\right)=\frac{\partial^{2} p}{\partial x^{2}}+\frac{\partial^{2} p}{\partial y^{2}}
$$

Where $p$ is pressure and $x$ and $y$ represent the grid point location. 
From each of the points the program searches for a local minimum pressure using an iterative approach. The (interpolated) first and second space derivatives of the pressure at a point are used to define an ellipsoid whose center becomes the next point in the iteration, as described in Murray and Simmonds (1991). Convergence occurs within three or four iterations.

An open system is searched for when a closed center cannot be determined by the ellipsoid. A system is classified as "weak/strong" based on the "concavity criterion" (Simmonds et al. 2008): when the average value of the Laplacian exceeds $0.2 \mathrm{hPa}\left({ }^{\circ} \text { lat }\right)^{-2}$ over a radius of $2^{\circ}$ lat, the storm is included in the count. If the Laplacian value is between 0.2 and $0.7 \mathrm{hPa}\left({ }^{\circ} \text { lat }\right)^{-2}$, the storm is considered "weak", and higher than $0.7 \mathrm{hPa}$ $\left({ }^{\circ} \text { lat }\right)^{-2}$, "strong". The depth is given in $\mathrm{hPa}$ and the Laplacian of the pressure is given in $\mathrm{hPa}\left({ }^{\circ} \text { lat }\right)^{-2}$, where one degree latitude is approximately $111 \mathrm{~km}$.

Feature points are then connected by estimating the subsequent displacement and pressure change of each cyclone at each timestep. The displacement is based on a weighting of the movement during the previous time step and the climatological zonally averaged cyclone velocities, the pressure change on a weighting of previous tendency, and persistence. The linkage is determined based on the combination of associations having the greatest overall probability. Even though Lagrangian methods have the same two processes - scanning to identify systems then the temporal tracking component - the way these processes are determined in the Melbourne algorithm involves other steps, and therefore they were described here. 
Statistics are then determined. Two important outputs from the Melbourne algorithm are the radius and depth, not given in any other tracking algorithm. The depth is given as the difference in pressures at the center and the "edge" of a cyclone (Simmonds; Keay 2009). The depth is an important parameter since it is related to the influence a cyclone has through the net meridional transport, for example. It can also serve as a measure of cyclone total kinetic energy because depth is approximately proportional to the square root of the total surface kinetic energy of a cyclone (and the surface stress), as described by Simmonds and Keay (2009):

$$
K E=\frac{\pi}{f^{2} \rho} D^{2}
$$

Where $K E$ is the kinetic energy, $f$ is the Coriolis parameter, $\rho$ is the density and $D$ is the cyclone depth. 


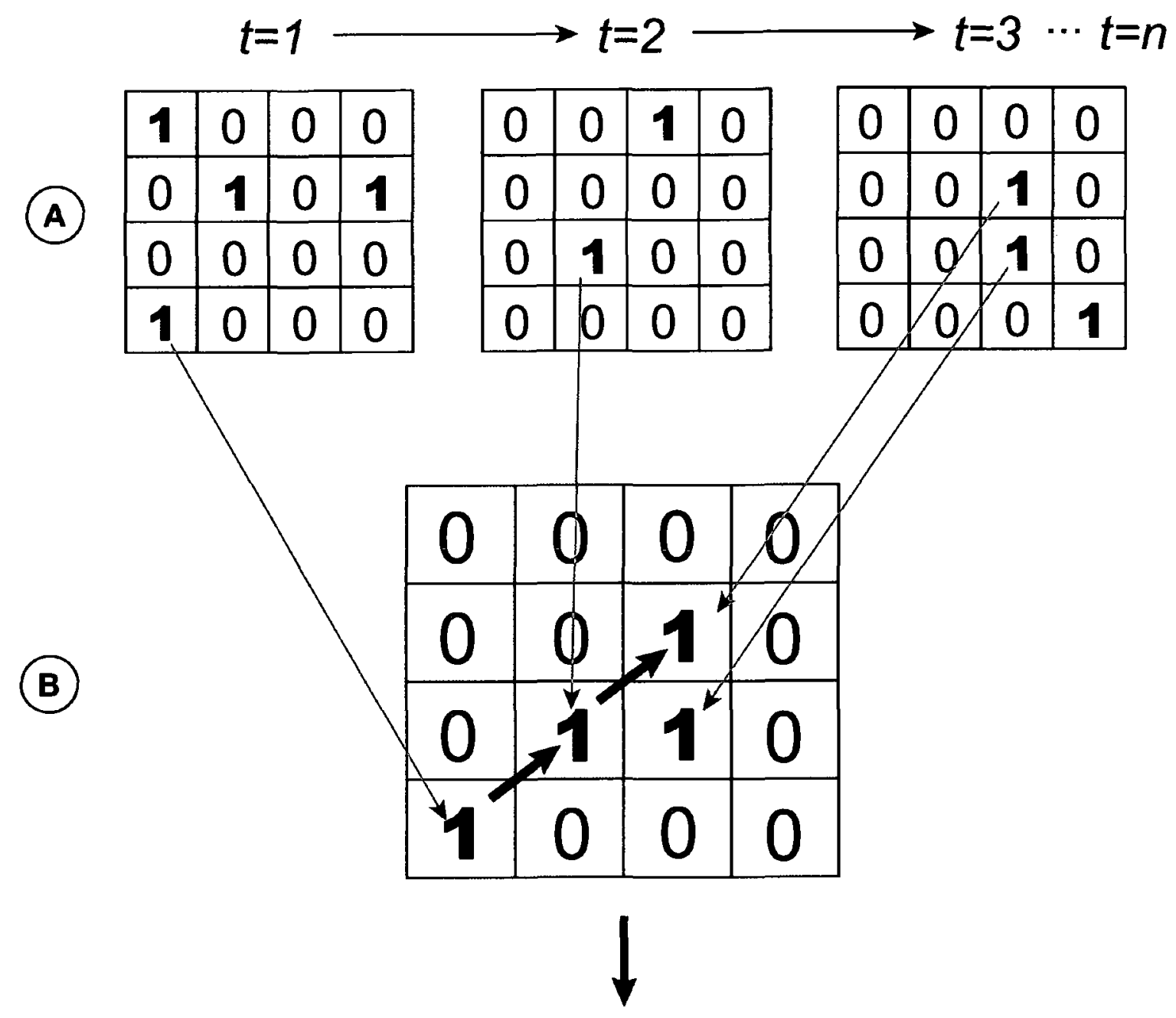

(C) Track Statistics (frequency/density)

Figure 3.1 - The tracking process

The three-way process to describe the tracking algorithms. 


\section{References}

Eichler, T., and W. Higgins, 2006: Climatology and ENSO-Related Variability of North American Extratropical Cyclone Activity. Journal of Climate, 19, 2076-2093.

Hodges, K. I., 1994: A General Method for Tracking Analysis and Its Application to Meteorological Data. Mon. Wea. Rev., 122, 2573-2586.

_- 1995: Feature Tracking on the Unit Sphere. Mon. Wea. Rev., 123, 3458-3465.

- 1996: Spherical Nonparametric Estimators Applied to the UGAMP Model Integration for AMIP. Mon. Wea. Rev., 124, 2914-2932.

- 1999: Adaptive Constraints for Feature Tracking. Mon. Wea. Rev., 127, 13621373.

Murray, R., and I. Simmonds, 1991: A Numerical Scheme for Tracking Cyclone Centres from Digital Data. Part I: Development and Operation of the Scheme. Aust. Met. Mag., 39, 155-166.

Serreze, M. C., 1995: Climatological Aspects of Cyclone Development and Decay in the Arctic. Atmos-Ocean, 33, 1-23.

Serreze, M. C., J. E. Box, R. G. Barry, and J. E. Walsh, 1993: Characteristics of Arctic synoptic activity, 1952-1989. Meteor. Atmos. Phys., 51, 147-164.

Serreze, M. C., F. Carse, R. G. Barry, and J. C. Rogers, 1997: Icelandic Low Cyclone Activity: Climatological Features, Linkages with the NAO, and Relationships with Recent Changes in the Northern Hemisphere Circulation. J. Climate, 10, 453-464.

Simmonds, I., and K. Keay, 2009: Extraordinary September Arctic sea ice reductions and their relationships with storm behaviour over 1979-2008. Geophys. Res. Lett., In press.

Simmonds, I., C. Burke, and K. Keay, 2008: Arctic Climate Change as Manifest in Cyclone Behavior. Journal of Climate, 21, 5777-5796. 
Zhang, X., J. E. Walsh, J. Zhang, U. S. Bhatt, and M. Ikeda, 2004: Climatology and Interannual Variability of Arctic Cyclone Activity: 1948-2002. J. Climate, 17, 23002317. 


\section{Chapter 4 Characteristics and Variability of Storm Tracks in the North Pacific,} Bering Sea and Alaska ${ }^{1}$

\section{Abstract}

The North Pacific and Bering Sea regions represent loci of cyclogenesis and storm track activity. In this paper climatological properties of extra-tropical storms in the North Pacific/Bering Sea are presented based upon aggregate statistics of individual storm tracks calculated by means of a feature-tracking algorithm run using NCEP/NCAR reanalysis data from $1948(49)$ to 2008 , provided by NOAA-CIRES. Storm identification is based on the $850-\mathrm{hPa}$ relative vorticity field (Vr) instead of the often used MSLP: $\mathrm{Vr}$ is a prognostic field, is a good indicator of synoptic-scale dynamics, and is directly related to the wind speed. Emphasis extends beyond winter to provide detailed consideration of all seasons.

Results show that the inter-seasonal variability is not as large during the spring and autumn seasons. Most of the storm variables - genesis, intensity, track density exhibited a maxima pattern that was oriented along a zonal axis. From season to season this axis underwent a north-south shift and, in some cases, a rotation to the northeast. This was determined to be a result of zonal heating variations and mid-tropospheric moisture patterns. Barotropic processes have an influence in shaping the downstream end of storm tracks and, together with the blocking influence of the coastal orography of northwest North America, result in high lysis concentrations, effectively making the Gulf

\footnotetext{
${ }^{1}$ Mesquita, M.d.S., D.E. Atkinson and K.I. Hodges, 2009: Characteristics and Variability of Storm Tracks in the North Pacific, Bering Sea and Alaska. J. Climate, in press.
} 
of Alaska the "graveyard" of Pacific storms. Summer storms tended to be longest in duration. Temporal trends tended to be weak over the study area. SST did not emerge as a major cyclogenesis control in the Gulf of Alaska.

\subsection{Introduction}

The coast of Alaska is experiencing serious impacts due to erosion and inundation. The primary drivers are waves and surge generated by strong winds that are typically associated with storms. Understanding trends and dynamics of coastal erosion in Alaska is grounded in grasping both the dynamics and the climatology of storms in the North Pacific and Bering Sea and how they vary spatially and temporally (Atkinson 2005; Rachold et al. 2005). This issue extends beyond the physical processes of erosion environmental and societal impacts are equally severe (Graham; Diaz 2001) and includes maritime industries (fishing, shipping) and subsistence hunting activities. The motivation to improve regional-scale understanding of storm track dynamics and patterns is thus strong. Storms are also a means by which the atmosphere redresses sinks and sources of energy and momentum; analyses with these theoretical meteorological objectives in mind are important for aiding our understanding of large-scale climate dynamics (Murray; Simmonds 1991).

Various analyses of storm activity have appeared in the literature; most have focused on the Northern Hemisphere as a whole with the Arctic being separately targeted in some instances (Hoskins; Hodges 2002; Keegan 1958; McCabe et al. 2001; Serreze 1995; Sorteberg; Walsh 2008; Whittaker; Horn 1984; Zhang et al. 2004). Relatively few, 
however, have focused specifically on the North Pacific and Bering Sea regions (Graham; Diaz 2001; Norris 2000) apart from identifying the prominent North Pacific storm track. Less common still are studies that consider seasons other than winter - a summer discussion is unusual and autumn/spring, the shoulder seasons, are almost never explicitly considered. Autumn is examined in Key and Chan (1999), however they do not explicitly focus on the North Pacific.

Trends in storm activity have been examined in several papers. McCabe et al. (2001), using Serreze's sea-level pressure (SLP) based algorithm and NCEP Reanalysis data, studied storm track trends in the Northern Hemisphere for the period 1959-97. They found an increase in high-latitude winter cyclone frequency and a decrease in midlatitude winter cyclone frequency over this period. Graham and Diaz (2001), using NCEP Reanalysis data and their own algorithm also based on SLP, calculated trends in the NP (defined only as far north as $50 \mathrm{~N}$ ) and found an overall winter (DJF) cyclone frequency trend of 0.21 cyclones per year over the period 1948-98. Key and Chan (1999), using NCEP Reanalysis data, studied storm tracks using the tracking algorithm by Bell and Bosart (1989), which is based on the geopotential height at two levels $(1000 \mathrm{mb}$ and $500 \mathrm{mb}$ ). Interestingly, even as far back as 1989 , they found that the surface cyclone frequency in the Arctic had increased during all seasons (DJF, MAM, JJA and SON), while surface cyclone frequency in the midlatitudes had decreased, in both the NH and SH. They also found opposite signs in trends in $1000 \mathrm{mb}$ and $500 \mathrm{mb}$, which could indicate that the systems had become shallower. 
Storm activity analyses are generally derived from climatologies that are developed by establishing a definition of a "storm", accumulating counts of their occurrences, and extracting statistics to determine their climatological characteristics (e.g. wind speed, ground speed, duration). Analyses of storm activity, such as reviewed above, have used different methods to track the storms, which vary in perspective (Lagrangian, Eulerian), method (manual tracking, automated tracking), variable (mean sea level pressure, geopotential height, relative vorticity, quasi-geostrophic vorticity, etc.) and tracking method (finding the central pressure in an MSLP field, finding the maxima in the relative vorticity field, finding the storm center by analyzing satellite imagery). Considering studies that have included the North Pacific (NP) the following range of approaches may be noted: Graham and Diaz (2001) used their algorithm based on SLP from a Lagrangian perspective; Norris (2000) used the root-mean-squared daily vertical velocity at $500 \mathrm{mb}$ during the summer season to diagnose storm tracks in the NP; Simmonds and Keay (2002) used the Melbourne University cyclone finding and tracking scheme (Murray; Simmonds 1991) based on the bi-cubic spline of the MSLP.

The key starting point in any analysis of storm activity is the event-level definition of a "storm". The most common meteorological variable used for this purpose has been MSLP, implemented in manual (Pickart et al. 2008; Whittaker; Horn 1984) and various automated tracking schemes (Murray; Simmonds 1991; Serreze et al. 1997; Zhang et al. 2004). Another meteorological variable that has been used is relative vorticity (५). Sinclair (1994), using the method of Murray and Simmonds (1991), is one of the first to use vorticity as a tracking variable. Sinclair makes an important point about 
the poleward displacement of the pressure minimum relative to the vorticity maxima in the presence of a background zonal wind field, which is an advantage of the method. In particular, the method defined by Hodges $(1994,1995,1996,1999)$ has also been found to yield satisfactory results for several reasons (Barry; Carleton 2001; Hoskins; Hodges 2002). First, contrasted with MSLP-based approaches, relative vorticity is more sensitive to small, rapidly-moving systems because MSLP tends to preferentially identify larger, slower-moving systems. In a geostrophic sense, vorticity focuses more on the small spatial-scale end of the synoptic range so that more systems are identified than by using MSLP, for example, which focuses more on the large-scale end (Hodges et al. 2003). This is important for high latitude work where small-scale features can occur with greater frequency than in the mid-latitudes (Twitchel et al. 1989).

Second, MSLP can be adversely influenced by the background flow. The position of a storm center represented by the MSLP may be biased by other fields superimposed in that one that can conceal its presence until farther along in its life-cycle (Hoskins; Valdes 1990). As well, a low pressure center situated within larger region of low pressure can possess a weak pressure gradient which means surface winds are weak (Fig. 4.1). The algorithm would identify and categorize the system as strong due to a low absolute pressure reading (e.g.: 956mb in Fig. 4.1), however the pressure gradient in the vicinity of the low pressure center is not very strong. Third, the presence of relative vorticity $(\mathrm{Vr}$ or $\zeta$, hereafter) is an important mechanism in the initiation of extra-tropical storms. Its use thus enables identification of a storm earlier in its lifecycle (Sorteberg; Walsh 2008) which can aid understanding of genesis dynamics. Fourth, $\mathrm{Vr}$ is important to the 
dynamical maintenance and growth of a system, so its use also facilitates on-going system monitoring. Finally, relative vorticity is also related directly to wind speed because its derivation is simply the curl of the vertical component of the relative velocity, that is:

$$
\zeta \equiv \mathbf{k} \cdot(\nabla \times \mathbf{U})=\frac{\partial v}{\partial x}-\frac{\partial u}{\partial y}
$$

Linking storm track information results directly to winds also improves their utility for secondary studies (e.g. coastal erosion) that depend upon wind forcing. For these reasons, therefore, storm climatology results based on Hodges' algorithm form the bases of the analyses presented here.

An important point that has often been overlooked in storm climatology analyses, especially with regards to impacts in northerly latitudes, is autumn activity. Autumn is an important time of year in the Arctic because it is the time of least ice and therefore greatest open water exposure (See Fig. 4.12, where the zero-degree isotherm provides a functional indication of the average sea-ice extent). In the region of study for this paper, autumn is also a time when the jet stream position and the occurrence of remnant tropical cyclone activity can create a particularly strong storm response.

Thus, it is the objective of this paper to aggregate storm activity results for the North Pacific and Bering Sea regions obtained using a robust and proven storm tracking algorithm, focusing on the seasonal time frame, including autumn. It also explores trends in storm activity responses, including trend considerations of pre and post- 1979 and 
discussions of changes in SST. Changes in horizontal gradients of SST affect baroclinic instability, which in turn influences storm tracks. Seasonal climate over the Pacific has also undergone changes in the mid to late 1970s (Graham; Diaz 2001). This analysis (pre- and post- 1979) will be conducted via a thorough discussion of different track variables, making this effort one of the most complete and updated studies of storm tracks for the North Pacific/Bering Sea.

Hence, the present paper is aimed at describing and understanding the climatology and trends of the storms in the North Pacific region, including the Bering Sea and the Gulf of Alaska, for standard climatological seasons, that is, winter (DJF), spring (MAM), summer (JJA) and autumn (SON). The following questions will be addressed:

a. Has there been a relative change in the seasonal patterns of storminess in these regions?

b. What are the characteristics of shoulder season storms? This question is important since spring and autumn are not typically addressed yet many of the most problematic storms in the Alaska region occur in the autumn.

c. What is the ground track speed of storms? The threat presented by a storm is increased if it is slow moving. Knowing how fast systems move is an important objective.

Section 4.2 discusses the methodology used; section 4.3 presents the results, focusing on a seasonal inter comparison of the climatologies and then on a discussion of the trends in the tracking variables; finally, even though a direct comparison with 
magnitudes from other climatologies is not immediately possible given the different normalizations in other studies (Simmonds; Keay 2002), a discussion will conclude the paper that places these results in light of the existing literature whenever possible. Due to space constraints, detailed consideration of these patterns in the context of other major circulation mode indices will be considered in another paper.

\subsection{Methodology}

Storm systems were identified and tracked using the algorithm developed by Hodges $(1994,1995,1996,1999)$ and run for the NH and subset for this study. In this algorithm relative vorticity is analyzed directly on the unit sphere and not on a preprojected or unprojected Cartesian grid. This approach "reduces the likelihood of introducing bias into the tracking and statistics and makes the parameters required for the tracking and statistical estimation independent of any projection" (Hodges et al. 2003). Essentially, using a gridded data set as input, the algorithm identifies a storm center to be a relative vorticity maximum that exceeds $1 \times 10^{-5} \mathrm{~s}^{-1}$. In order to solve the correspondence between the feature points in consecutive time steps, which Hodges identifies as the most difficult aspect of any storm tracking system, a specialized cost function is applied (Hodges 1995). Minimization of the cost function results in the smoothest set of tracks subject to constraints on displacement distance and the track smoothness.

Relative vorticity at $850-\mathrm{hPa}$ is used as the analysis variable. $850-\mathrm{hPa}$ represents a good tropospheric weather activity level for storm tracking (Hodges et al. 2003). Likewise vorticity represents a good analysis variable because it does not depend on any 
extrapolation techniques, such as the MSLP. The tracks have been filtered to retain only those that last longer than 2 days and travel further than $1000 \mathrm{~km}$ (mobile storms).

The algorithm input data, $4 \mathrm{x}$ daily $\mathrm{u}$ and $\mathrm{v}$ for the relative vorticity calculation, were drawn from the NCEP/NCAR global reanalysis (R1) (Kalnay et al. 1996; Kistler et al. 2001) from 1948/49-2008 provided by NOAA/NWS/NCEP (NCEP Reanalysis data provided by the NOAA/OAR/ESRL PSD, Boulder, Colorado, USA, from their Web site at http://www.cdc.noaa.gov/). Skin Surface Temperature (SST) data were provided by the NCEP/NCAR Reanalysis as well.

Six tracking output variables were retained for analysis:

i. Genesis density (gdens) - it shows the areas where the storm tracks start per season. Units: density of starting points $\left(10^{6} \mathrm{~km}^{2} \text { season }\right)^{-1}$;

ii. Track density (tdens) - Number of storm tracks through a region per season. Units: storms $\left(10^{6} \mathrm{~km}^{2} \text { season }\right)^{-1}$;

iii. Lysis density (ldens) - it shows the areas where the storm tracks die out per season. Units: density of ending points $\left(10^{6} \mathrm{~km}^{2} \text { season }\right)^{-1}$;

iv. Intensity (int) - Intensity of the vortex. Units: intensity in $10^{-5} \mathrm{~s}^{-1}$;

v. Mean lifetime $(l f t)$ - the number of days the low pressure systems lasted that pass through a region;

vi. Mean Speed $(m s p d)$ - The mean track speed in $\mathrm{m} \mathrm{s}^{-1}$.

Two major analyses were conducted on each of these variables. First, a general pattern analysis by season was conducted for the six tracking variables. Second, assessment of trends using least squares fitting for selected variables was performed for 
three collective regions in and around Alaska (Fig. 4.2). Statistical significance was based on the t-test of the linear regression coefficients. The regions studied were:

i. Alaska Interior/Yukon ('AIY') - From latitude $60 \mathrm{~N}$ to $70 \mathrm{~N}$ and from longitude $160 \mathrm{~W}$ to $120 \mathrm{~W}$;

ii. Gulf of Alaska ('GOA') - From latitude $50 \mathrm{~N}$ to $60 \mathrm{~N}$ and from longitude $160 \mathrm{~W}$ to 120W;

iii. Bering Sea ('BeS') - From latitude $50 \mathrm{~N}$ to $70 \mathrm{~N}$ and from longitude $160 \mathrm{E}$ to $160 \mathrm{~W}$.

The next section will discuss the main results of these analyses. 


\subsection{Results}

\subsubsection{Climatology}

The overall pattern in the cyclogenesis density plots (Figure 4.3, A-D) show two major storm initiation maxima: the North Pacific and the North American continental interior. The North Pacific maximum, oriented east-west, is situated in response to the zone of strong baroclinicity that arises from a strong and persistent poleward temperature gradient. This maximum is referred to as 'secondary cyclogenesis' in Hoskins and Hodges (2002). The strong formation area over the North American continental interior is largely a result of lee cyclogenesis behind the coastal mountain ranges, that is, cyclogenesis initiated by the conservation of potential vorticity (Holton 2004). It has been suggested that the formation area over the northern continent is also associated with the land-sea temperature contrast (Serreze et al. 2001). The largest values of cyclogenesis are found in the regions bounded by $35-45 \mathrm{~N}$ and $160 \mathrm{E}-150 \mathrm{~W}$, over the central North Pacific, and over northwestern Canada during winter; spatial patterns in other seasons are generally similar with a reduction in cyclogenesis magnitude. Generally the Pacific track parallels lines of latitude. The maxima axes shift north and south with the seasons farthest south in winter and farthest north in summer. In summer there is a distinct departure from the linear east-west pattern with the appearance of a west-northwest tending kink or dogleg in the axis, such that the axis shifts and rotates to run roughly parallel to the eastern half of the Aleutian Islands. This is also evident to a weaker extent as a component of the autumn axis pattern. 
Over Alaska, cyclogenesis density is greatest in winter, becoming more confined to the central/northern part of Alaska during the other seasons. A similar reduction is observed over a small cyclogenesis zone over the Chukotkta north coast. By summer, cyclogenesis is present at low magnitudes over Alaska and Chukotka, with the lee cyclogenesis zone persisting east of the Canadian Rockies. Overall, summer is the season of lowest cyclogenesis activity over the study area. The autumn cyclogenesis pattern is similar to spring, with the exception of extensions of the Pacific genesis zone into both the Kamchatka Peninsula/Sea of Okhotsk area and well up into the Gulf of Alaska. These extension features are unique to autumn.

The overall track density pattern (Fig. 4.4 A-D) indicates a favored pathway running east-west in the North Pacific. A persistent collection zone in the Gulf of Alaska was also present in all seasons. Overall, track densities are highest in winter - peaking at $37-45$ storms $\left(10^{6} \mathrm{~km}^{2} \text { season }\right)^{-1}$, although autumn has a similar magnitude peak - and lowest in summer - peaking at 13-29 storms $\left(10^{6} \mathrm{~km}^{2} \text { season }\right)^{-1}$. During winter, the zone of storm frequency maxima covers most of the North Pacific and the Gulf of Alaska. Spring and autumn track densities have characteristics similar to one another - both have a similar core within the broader Pacific maximum, stretching over the North Pacific towards the Gulf of Alaska with a peak density of exceeding 37 storms $\left(10^{6} \mathrm{~km}^{2}\right.$ season) 1. The seasonal north/south shift in the maxima axis, observed for gdens, is observed again here - in summer the storm track lies along a more northerly position, closer to the Aleutian Islands. The dogleg feature observed in genesis density was observed again for track density, most prominently in the winter, then less so in fall, spring, and finally not 
apparent in the summer. Spring and autumn count maxima exceeding $37 \mathrm{storms}\left(10^{6} \mathrm{~km}^{2}\right.$ season $)^{-1}$ are confined to specific regions - in the spring the maximum is located mainly in the region $150-170 \mathrm{E}$, whereas during autumn this threshold is exceeded over a more broadly distributed region across the North Pacific, including a large zone in the Gulf of Alaska. Activity over continental regions is much lower, with mainland Alaska never exhibiting more than 20 storms $\left(10^{6} \mathrm{~km}^{2} \text { season }\right)^{-1}$ in any season, with slightly more activity in autumn and winter.

The Gulf of Alaska emerges as a strong and consistent location for storm lysis, and may be considered the "Graveyard of Pacific Storms" (Figure 4.5 A-D). During winter, lysis density is widespread over the GOA, extending far enough south to present a second maximum over Vancouver Island. Lysis density is lowest in the summer and highest in fall. This could be due to the fact that summer storms last longer, and thus are able to move further from the Gulf of Alaska and the North Pacific; as well, the storm count, as reflected by tdens, is lower. Spring and autumn lysis densities exhibit some similar patterns, with autumn having slightly larger maxima values. The Bering Sea/Bering Strait areas also present high lysis density, especially in winter and autumn.

Summer storms last longer than winter ones. This is especially true over most of the North Pacific and the Gulf of Alaska (Figure 4.6 A-D). Winter storms typically last in excess of 4.5 days over the western North Pacific. In spring, storm duration increases to about 5.5 days. In summer storm duration exceeds 5.5 days over most of the North Pacific and the Gulf of Alaska. The large region of Pacific lifetime maxima ( $>6$ days) 
exhibited in summer is reduced in autumn to a narrower and shorter band extending northeast to the Aleutian Islands.

The intensity results are based on the filtered vorticity intensities, i.e. the T42 intensities with background removed, as discussed in Hoskins and Hodges (2002). Winter storms are most intense (Figure 4.7 A-D); summer intensities are lowest. Spring and autumn track intensity patterns and magnitudes are quite similar to winter. Winter intensity maxima are situated south of the Bering Sea and Gulf of Alaska. These maxima features are reduced in the other seasons. The summer intensity pattern is also shifted slightly northwards and is narrower compared to the other seasons. Unlike the other parameters, for the most part the zone of greatest intensity remains consistently confined to the central Aleutians and south.

Storms move most rapidly in the winter (Figure 4.8 A-D), tracking with an average speed of about $14 \mathrm{~m} \mathrm{~s}^{-1}$ over most of the North Pacific, with a maxima west of $160 \mathrm{~W}$ along $35 \mathrm{~N}$ where mean track speed exceeds $15.5 \mathrm{~m} \mathrm{~s}^{-1}$. Spring and autumn patterns are similar to winter; magnitudes are lower. Summer storms are slowest compared to the other seasons, moving at a typical speed of less than $12.5 \mathrm{~m} \mathrm{~s}^{-1}$ over most of the North Pacific, with a maxima core of $11-12.5 \mathrm{~m} \mathrm{~s}^{-1}$ located where the spring and autumn cores are. 


\subsubsection{Trends}

In this section detailed temporal analyses are presented for three variables: genesis density (gdens), lysis density (ldens) and a new variable defined for this particular assessment: a count of storms which enter the region in question (enter) - in Table 4.1. These are analyzed for the period pre and post-1979. Intensity and track density trends are examined using separate plots (Figs. 4.9, 4.10 and 4.11). Commentary is restricted to significant trends only (above $90 \%$ significance levels).

The Alaska interior exhibited six statistically significant trends (Table 4.1). Two trends were exhibited for the period 1948(49) to 2008: the spring gdens $(+0.93 \%$ per year) and winter enter (-0.44\%). The period between 1948(49) to 1978 presented no statistically significant trends. The period from 1979 to 2008 possessed four statistically significant trends: winter gdens (-1.55\%), summer gdens (-1.54\%), autumn gdens ($1.18 \%)$, and the autumn enter $(-3.43 \%)$.

The Gulf of Alaska exhibited five statistically significant trends for 1948(49) 2008 (Table 4.1), reviewed below (rates are \% per year). Gdens exhibited trends in winter $(-0.45 \%)$ and summer $(-0.40)$. Ldens exhibited trends in winter $(+0.55 \%)$, spring $(+0.59 \%)$, and summer $(+0.32 \%)$. Three trends are significant during the period 1948(49) to 1978: gdens in winter (-1.15\%) and summer $(-2.09 \%)$, and ldens in summer $(+1.08 \%)$. One statistically significant trend was apparent for the third period $(1979-2008)$ : summer gdens $(-1.98 \%)$.

Finally, the Bering Sea presented four statistically significant trends: two in the period $1948(49)$ to 2008 spring ldens $(-0.29 \%)$ and spring enter $(-0.24 \%)$. The period 
from $1948(49)$ to 1978 shows no statistically significant trends. The period $1948(49)$ to 2008 shows two trends: summer ldens $(-0.76 \%)$ and autumn ldens $(+0.79 \%)$.

Two other important variables for Alaska are the track density and the track intensity. The track density decadal anomaly patterns show large departures from the mean in the last decade (1999-2008 - Fig. 4.9). The largest anomalies are observed in these decadal periods: 1949-1958, 1979-1988 and 1999-2008. Anomalies in the Gulf of Alaska are positive in the last three decadal periods (i.e. since 1979). In the Alaska interior a change in anomaly sign also took place after 1979 , represented by an increase from 1979-1998 and a decrease in the past decade. As for the Bering Sea, the first two decades show a tdens lower than mean and the past decades, higher.

Intensity (int) is examined using seasonal histograms from 1948(49) - 2008 (Fig. 4.10). Here, these intensities are the full resolution intensities obtained by referencing the tracks back to full resolution, computed in the same way as in Bengtsson et al. (2009), by searching for a maximum within a 5 degree spherical cap region. In the Gulf of Alaska and Bering Sea the most frequently occurring intensity magnitude ranged around $6 \times 10^{-5}$ $\mathrm{s}^{-1}$, whereas in the Alaska interior intensity magnitudes are lower, ranging around 3-4x $10^{-}$ ${ }^{5} \mathrm{~s}^{-1}$ for all seasons. In the Gulf of Alaska and Bering Sea the most frequent values shift between 4-7 $\times 10^{-5} \mathrm{~s}^{-1}$ in the summer and spring up to $5-10 \times 10^{-5} \mathrm{~s}^{-1}$ in autumn and winter. At the highest end of the scale the Bering Sea has in each season a greater number of higher intensity events than the Gulf of Alaska, including a greater number exceeding $12 \times 10^{-5} \mathrm{~s}^{-1}$. The Alaska interior does not experience such events; maximum intensities are $10 \times 10^{-5} \mathrm{~s}^{-1}$. By season, winter and autumn exhibited the highest number of extreme 
event cases, commensurate with results already presented. Summer has the fewest number of extreme events and it is the only season in which the histogram of the Gulf of Alaska and the Bering Sea differ the most. The broadest observation from these results is that in every season the Bering Sea has more, higher intensity events than the Gulf of Alaska.

A closer look at the winter intensity histogram by decade (Fig. 4.11) shows the properties described for figure 4.10 are echoed at this higher temporal resolution. The Alaska interior seems to have had an average of about 1.2-1.6 storms per month for its peak intensity at around $3 \times 10^{-5} \mathrm{~s}^{-1}$. The Gulf of Alaska peak number of events also increased. In the first decades, an average of about $6 \times 10^{-5} \mathrm{~s}^{-1}$ was observed. In the past decades, this value has increased to about $9 \times 10^{-5} \mathrm{~s}^{-1}$. The Bering Sea average of $6 \times 10^{-5} \mathrm{~s}^{-}$ ${ }^{1}$ for its peak number of events seem to have slightly increased to about $9 \times 10^{-5} \mathrm{~s}^{-1}$ until the fourth decade, decreased in the final two. The histogram changed in the past two decades. The number of extreme events for the Gulf of Alaska and Bering Sea (intensity $>12 \times 10^{-5} \mathrm{~s}^{-1}$ ) increased in the past decade. The Bering Sea had a consistently high number of extreme events through the different decades. 


\subsection{Discussion and Conclusion}

Winter emerged as the most active season for most of the storm parameters, including genesis density and track density. Simmonds and Keay (2002) found high winter mean cyclone occurrences (tdens) over the Sea of Okhotsk and the Gulf of Alaska, which is essentially in accordance with our results, except that we find even higher tdens counts during autumn (a season not considered in their study). Winter storm tracks are also located farther south, a result consistent with many studies (Nakamura 1992; Simmonds; Keay 2002; White 1982). This location is also influenced by the midwinter suppression (Nakamura 1992). One of the key determinants of storm activity is a strong thermal gradient. The north-south gradient of temperature is greatest in the winter. The sea ice extent is farthest south in late winter (Fig. 4.12). In addition, the Kuroshio Extent (Qiu 2000), also known as the North Pacific current, acts as a southern anchor for the thermal gradient. The net effect results in a thermal gradient that is strongest in winter. This is conducive to more frequent episodes of baroclinic instability that favor development, growth, and energy levels of extra-tropical cyclonic systems as is evidenced by descriptions in the Results section. An analysis of mean spatial patterns of the index of Eady baroclinic growth rate (not shown) also indicates a maximum zone that is coincident with fall and winter storms.

The role of diabatic heating is strong in the generation of storms off the east coast of Asia and North America, providing another mechanism for generating the strong thermal gradient mentioned above, which supports the Pacific/Atlantic maxima (Hoskins; Valdes 1990). Release of latent heat also plays an important role in the 
intensity of the low pressure systems and the rapidity of cyclogenesis (Chang et al. 1982; Posselt et al. 2008). In a sense, the NP (and North Atlantic) storm tracks may be considered as self-maintaining, since the diabatic heating maxima in the storm track region are caused by horizontal and vertical flow displacements which are in turn a byproduct of the individual storms (Hoskins; Valdes 1990). Storm tracks can be selfmaintained also by the fact that they help drive the Kuroshio current due to the low-level mean flow induced by the eddy activity, and in turn, the Kuroshio current is crucial for the existence of the NP maximum (Hoskins; Valdes 1990).

The lysis density analysis results obtained here reinforces the concept that the Gulf of Alaska is the "graveyard" of storms in the NP as pointed out, for example, by Simmonds and Keay (2002). The tdens results (Fig. 4.4) suggest that most of the storm systems that end up in the Gulf of Alaska do not form locally. A targeted assessment verifies this fact (Fig. 4.13), which indicates that most storms are not forming locally with the exception of the summer season, for which a source region in the Aleutian Islands is indicated. Also, the mid-ocean cyclogenesis is likely due to secondary cyclogenesis and downstream development as discussed in Hoskins and Hodges (2002). This may also be roughly concluded by realizing that the mean track speed $(12 \mathrm{~m} / \mathrm{s})$ and lifetime ( 5 days) of these storms (winter considered) results in a lifetime distance traveled of approximately $5000 \mathrm{~km}$. Another important aspect is the fact that there are indications of tropical cyclones undergoing extra-tropical transitions and reaching GOA, especially during summer and autumn (Fig. 4.14). 
Once in the Gulf of Alaska, there are several mechanisms at work that contribute to the large observed lysis values. First, barotropic processes typically are the dominant dynamical mechanisms at work. Understanding the role played by barotropic processes is important, since the life cycles of baroclinic waves in a zonally symmetric flow are characterized by positive conversions in the growth phase and negative barotropic conversions in the decay phase (Frisius et al. 1998; Simmons; Hoskins 1978). This way, barotropic processes play an important role in the lysis phase of the storm tracks. Second, and perhaps exerting greater importance, the role of regional topography must be considered. The topography of coastal Alaska and northwest North America - the coastal highlands and mountains - is manifested as an influencing agent because its physical presence could be great enough to help destroy shear.

The shear of the meridional wind and the zonal wind contribute equally to the total shear production (destruction) (Frisius et al. 1998). Negative shear production has been shown to be responsible for the location of the 'downstream end' of the storm track (Frisius et al. 1998; Whitaker; Dole 1995). Shear destruction of the transient eddies (barotropic processes) associated with lysis reduces the TKE because via this process, eddies release $\mathrm{KE}$ to the mean flow $(\mathrm{TKE}=$ advection + baroclinic production + shear production (destruction) + dissipation) and the storms die out, in this case, in the Gulf of Alaska (Black; Dole 2000; Frisius et al. 1998).

Another factor at work in the NP context is the presence of a favored location for a downstream blocking ridge over the Aleutians/Alaska region, also known as the 'Alaska block' pattern (Frisius et al. 1998; Lau 1988), that can act to retard the eastward 
progression of systems along the Pacific storm track. According to Simmonds and Keay (2002), the Gulf of Alaska is also a region of high cyclone frequency; however, this is not incompatible with high lysis values because GOA is also an area of strong baroclinicity, indicated by the Eady growth rate assessment, which can enhance activity, and because systems can become stalled against topography or blocking patterns, mentioned above, which could enhance residence time. Thus both the barotropic deformation (D) and the tropospheric baroclinic growth (B) could play an important role in the dynamics over the Gulf of Alaska, as it is illustrated by the ratio of the D over the B over GOA, as shown by Black and Dole (2000). Such a result is consistent with a relatively high Eady growth rate, an indicator that is proportional to vertical shear.

As for the lifetime, summer storms last longer than any other season. This result expands upon two previous studies (Mesquita et al. 2008; Zhang et al. 2004) that confined comparisons of lifetime to summer and winter. Summer storms are also the least intense, with winter, spring and autumn intensities being greater and similar to one another. Simmonds and Keay (2002) also found that, in general, the mean intensity is significantly reduced in summer with the most intense summer systems found over and southwest of the Aleutian region, also in accordance with our results. Their intensity plots, using a different measure of intensity, of the winter and summer intensity resemble our intensity DJF and JJA plots as well. Finally, the mean speed of the storms is higher in winter. In summary, summer storms last longer, move more slowly and are less intense. A common denominator linking these traits together is the growth rate (not shown). Summer storms tend to have much lower growth rates. One import feature of the 
climatology analysis is that the transitional seasons, spring and autumn, tended to have similar values for most of the storm parameters considered.

Whitaker and Dole (1995) mention that storm tracks exhibit considerable variability in interannual and intraseasonal timescale. We agree that the interannual variability is considerable, and are able to shed more light on the inter-seasonal issue, but we have found a different result in our study concerning the inter-seasonal aspect: spring and autumn present similar characteristics for all of the variables with the exception that autumn int is stronger than spring. Small differences in lft and lysis were also noted (autumn was larger in both cases). The greatest contrasts are observed during winter and summer, which are the times of largest contrasts in temperature gradients. Summer storms have the lowest values for gdens, tdens, int, and lysis. Summer storms, however, last longer than winter ones. This is in accordance with Zhang et al. (2004) and Mesquita et al. (2008). This result is expanded here to show that they last longer than any of the other seasons. The track density (tdens) results by decade (Fig. 4.9) underscore the strong interannual and interdecadal variability that work against isolation of a trend signal.

Maxima spatial patterns exhibited a north/south shift. The main factor responsible for the maintenance of this zonal variation of baroclinicity is the distribution of heating (Frisius et al. 1998; Hoskins; Valdes 1990). A rotation "point" in the central Aleutian Islands, as for example in the gdens, tdens and mspd cases, was also apparent. This dogleg in the storm tracks observed here is caused in part by the temperature gradient changes in the North Pacific but is also suspected to be a by-product of a mid- 
tropospheric $(700 \mathrm{mb})$ moisture pattern in which the Bering Sea region is dryer than the Gulf of Alaska in all seasons except summer (analysis not shown).

Of the three aggregate regions considered in this paper, AIY has higher annual gdens compared to the other regions. GOA has higher number of storms (tdens) and higher ldens. BeS shows storms with higher lifetime, intensity and speed. When considering storms with intensities exceeding $12 \times 10^{-5} \mathrm{~s}^{-1}$, however, BeS was found to consistently possess more of these high intensity storms than the GOA (Fig. 4.10). The Eady growth rate analysis indicated a zone of high Eady potential south of Russia's Kamchatka Peninsula, which can serve to re-energize older systems as they move into BeS. It is hypothesized here that, by the time systems enter GOA, the increasing shear regime coupled with greater downstream distance from the Kamchatka Eady max precludes stronger development in most cases.

In general, most of the storm parameters trends observed in this study were not very robust. Most of the statistically significant trends at the $99 \%$ s.l. are related to the gdens, for the AIY region and gdens and ldens for the BeS. The fact that different trends are found for the periods before and after 1979 reflect an underlying factor in this case: the influence of large modes of climatic variability, which may play an important role in such trends. The issue of fleeting statistical significance speaks to both a few degrees of freedom issue caused by the relatively short time series available to work with and the large interannual variability observed in these variables. A possible additional source of pre- and post-1979 variability could be due to the introduction of large quantities of satellite data into the reanalysis after 1979 and which has progressively increased with 
time (Bengtsson et al. 2004; Bromwich et al. 2007). There are a variety of points to make about the trends results, however.

When considering the trends for the three regions and the three different periods of time - the entire 1948-2008 timeseries and pre- and post 1979 - we observe that most of the trends that are statistically significant (at the $95 \%$ and $99 \%$ significance levels) occur in the GOA region, generally for genesis density and lysis density. An interesting point is that Latif and Barnett (1996) showed strong positive SST trends for GOA. Positive SST trends and SST gradients are also observed in the NCEP data (not shown). Despite this, strong positive trends are not noted for those storm parameters that would be influenced by SST forcing, namely genesis density - gdens is decreasing wherever its trend is significant. Hence, SST does not appear to be a key storm-forcing agent in the Gulf of Alaska. Some studies have related the sea ice cover in the Sea of Okhotsk to the location and intensity of the Aleutian Low - which could play an important role in the GOA trends. For example, Honda et al. (1999) suggest a significant influence of ice cover on the downstream pressure field. Few strong, significant trends were noted for the Alaska interior. As for the Bering Sea, not many trends were found there.

Two variables are of particular importance for Alaska when it concerns trends: the number of storms (tdens or enter) and their intensity (int). None of the three regions considered show trends for these variables. Other studies have suggested "storms are increasing". For example, McCabe et al. (2001), using Serreze's tracking algorithm (Serreze 1995), found a considerable increase in cyclone frequency in high latitudes (60$90 \mathrm{~N})$ and a pronounced decrease in midlatitudes $(30-60 \mathrm{~N})$. The results of McCabe et al. 
represented broadly integrated swaths. Our results, however, indicate that, for the regions considered here, this is not really the case: there is no particular trend in storm activity in the AIY, BeS, or GOA regions.

As for the intensity, this result also appears to contrast with the paper by McCabe et al. (2001) who found that the storm intensity has increased both in mid and high latitudes. Graham and Diaz (2001) found an intensification of NP winter cyclones which resulted from the increasing upper-tropospheric zonal winds between $25 \mathrm{~N}$ and $40 \mathrm{~N}$ associated with ENSO. It is not simple to compare trends in intensity with McCabe et al. (2001) and Graham and Diaz (2001) because of the different approaches to define and calculate the intensity variable. This could also be due to the use of pressure, which can be influenced by the large-scale background. And if intensity is measured as the pressure minima, as in Graham and Diaz (2001), it would be influenced by any large scale changes. However, the lack of intensity trends found in our study is consistent with the fact that these regions are a little north of the main path/track of the Pacific maximum, except the GOA, which is the region with high lysis density.

An interesting result is a clear indication that SST does not appear to be a strong storm control for the marine regions explicitly considered: Gulf of Alaska and Bering Sea. Instead, local shear and topography conditions appear to exert the greater control. This is important in light of potential future increasing trends in SST if sea ice continues to retreat, i.e., that storm activity will not follow suit because of SST alone. It is important to note that, overall, Bering Sea trends are difficult to identify in part because of high inter-annual variability. The relative lack of influence of SST also shows up in 
the lysis values that remain relatively consistent all along the west coast, even in the presence of considerable SST gradients. 


\section{Acknowledgments}

This research was funded by the National Oceanic and Atmospheric Administration (NOAA) under the project title: "Social Vulnerability to Climate Change in the Alaskan Coastal Zone" (grant NA06OAR4600179). We are grateful for NCAR for providing their data sets in the public domain. We would like to thank Dr. John Walsh, from the International Arctic Research Center at the University of Alaska Fairbanks, for the invaluable comments on the paper. We also thank Dr. Asgeir Sorteberg, from the University of Bergen (Norway), and Christine Radermacher, from the University of Bonn (Germany) for the initial data set up discussions. We appreciate comments made by the anonymous reviewers. This is publication no. A 248 from the Bjerknes Centre for Climate Research. 


\section{References}

Atkinson, D. E., 2005: Observed Storminess Patterns and Trends in the Circum-Arctic Coastal Regime. Geo-Mar. Lett., 25 (2-3), 98-109.

Barry, R. G., and A. M. Carleton, 2001: Synoptic and Dynamic Climatology. Routledge, $620 \mathrm{pp}$.

Bell, G. D., and L. F. Bosart, 1989: A 15-Year Climatology of Northern Hemisphere 500 mb Closed Cyclone and Anticyclone Centers. Mon. Wea. Rev., 117, 2142-2164.

Bengtsson, L., S. Hagemann, and K. I. Hodges, 2004: Can climate trends be calculated from reanalysis data? J. Geophys. Res., 109, D11111.

Bengtsson, L., K. I. Hodges, and N. Keenlyside, 2009: Will Extratropical Storms Intensify in a Warmer Climate? J. Climate, 22, 2276-2301.

Black, R. X., and R. M. Dole, 2000: Storm Tracks and Barotropic Deformation in Climate Models. J. Climate, 13, 2712-2728.

Bromwich, D. H., R. L. Fogt, K. I. Hodges, and J. E. Walsh, 2007: A tropospheric assessment of the ERA-40, NCEP, and JRA-25 global reanalyses in the polar regions. $J$. Geophys. Res., 112, D10111.

Chang, C. B., D. J. Perkey, and C. W. Kreitzberg, 1982: A Numerical Case Study of the Effects of Latent Heating on a Developing Wave Cyclone. J. Atmos. Sci., 39, 1555-1570.

Frisius, T., F. Lunkeit, K. Fraedrich, and I. N. James, 1998: Storm-track organization and variability in a simplified atmospheric global circulation model. Quart. J. Roy. Meteor. Soc., 124, 1019-1043.

Graham, N. E., and H. F. Diaz, 2001: Evidence for Intensification of North Pacific Winter Cyclones since 1948. Bull. Amer. Meteor. Soc., 82, 1869-1893.

Hodges, K. I., 1994: A General Method for Tracking Analysis and Its Application to Meteorological Data. Mon. Wea. Rev., 122, 2573-2586.

_ 1995: Feature Tracking on the Unit Sphere. Mon. Wea. Rev., 123, 3458-3465. 
- 1996: Spherical Nonparametric Estimators Applied to the UGAMP Model Integration for AMIP. Mon. Wea. Rev., 124, 2914-2932.

- 1999: Adaptive Constraints for Feature Tracking. Mon. Wea. Rev., 127, 13621373.

Hodges, K. I., B. J. Hoskins, J. Boyle, and C. Thorncroft, 2003: A Comparison of Recent Re-analysis Data Sets Using Objective Feature Tracking: Storm Tracks and Tropical Easterly Waves. Mon. Wea. Rev., 131, 2012-2037.

Holton, J. R., 2004: An Introduction to Dynamic Meteorology. 4 ed. Elsevier Academic Press.

Honda, M., K. Yamazaki, H. Nakamura, and K. Takeuchi, 1999: Dynamic and Thermodynamic Characteristics of Atmospheric Response to Anomalous Sea-Ice Extent in the Sea of Okhotsk. J. Climate, 12, 3347-3358.

Hoskins, B. J., and P. J. Valdes, 1990: On the Existence of Storm-Tracks. J. Atmos. Sci., 47, 1854-1864.

Hoskins, B. J., and K. I. Hodges, 2002: New Perspectives on the Northern Hemisphere Winter Storm Tracks. J. Atmos. Sci., 59, 1041-1061.

Kalnay, E., and Coauthors, 1996: The NCEP-NCAR 40-Year Reanalysis Project. Bull. Amer. Meteor. Soc., 77, 437-471.

Keegan, T. J., 1958: Arctic Synoptic Activity in Winter. J. Atmos. Sci., 15, 513-521.

Key, J. R., and A. C. K. Chan, 1999: Multidecadal Global and Regional Trends in 1000 $\mathrm{mb}$ and $500 \mathrm{mb}$ Cyclone Frequencies. Geophys. Res. Lett., 26, 2053-2056.

Kistler, R., and Coauthors, 2001: The NCEP-NCAR 50-year Reanalysis: monthly means CD-ROM and Documentation. B. Am. Meteorol. Soc., 82, 247-267.

Latif, M., and T. P. Barnett, 1996: Decadal Climate Variability over the North Pacific and North America: Dynamics and Predictability. J. Climate, 9, 2407-2423.

Lau, N.-C., 1988: Variability of the Observed Midlatitude Storm Tracks in Relation to Low-Frequency Changes in the Circulation Pattern. J. Atmos. Sci., 45, 2718-2743. 
McCabe, G. J., M. P. Clark, and M. C. Serreze, 2001: Trends in Northern Hemisphere Surface Cyclone Frequency and Intensity. J. Climate, 14, 2763-2768.

Mesquita, M. D. S., N. G. Kvamstø, A. Sorteberg, and D. E. Atkinson, 2008: Climatological properties of summertime extra-tropical storm tracks in the Northern Hemisphere. Tellus $A, 60$.

Murray, R., and I. Simmonds, 1991: A Numerical Scheme for Tracking Cyclone Centres from Digital Data. Part I: Development and Operation of the Scheme. Aust. Met. Mag., 39, 155-166.

Nakamura, H., 1992: Midwinter Suppression of Baroclinic Wave Activity in the Pacific. J. Atmos. Sci., 49, 1629-1642.

Norris, J. R., 2000: Interannual and Interdecadal Variability in the Storm Track, Cloudiness, and Sea Surface Temperature over the Summertime North Pacific. $J$. Climate, 13, 422-430.

Pickart, R. S., G. W. K. Moore, A. M. Macdonald, I. A. Renfrew, J. E. Walsh, and W. S. Kessler, 2008: Seasonal evolution of Aleutian low-pressure systems: Implications for the North Pacific sub-polar circulation. J. Phys. Oceanogr., preprint, 0000-0000.

Posselt, D. J., G. L. Stephens, and M. Miller, 2008: CLOUDSAT: Adding a New Dimension to a Classical View of Extratropical Cyclones. Bull. Amer. Meteor. Soc., 89, 599-609.

Qiu, B., 2000: Interannual Variability of the Kuroshio Extension System and Its Impact on the Wintertime SST Field. J. Phys. Oceanogr., 30, 1486-1502.

Rachold, V., F. E. Are, D. E. Atkinson, G. Cherkashov, and S. M. Solomon, 2005: Arctic Coastal Dynamics - An Introduction. Geo-Mar. Lett., 25 (2-3), 63-68.

Serreze, M. C., 1995: Climatological Aspects of Cyclone Development and Decay in the Arctic. Atmos-Ocean, 33, 1-23.

Serreze, M. C., A. H. Lynch, and M. P. Clark, 2001: The Arctic Frontal Zone as Seen in the NCEP-NCAR Reanalysis. J. Climate, 14, 1550-1567. 
Serreze, M. C., F. Carse, R. G. Barry, and J. C. Rogers, 1997: Icelandic Low Cyclone Activity: Climatological Features, Linkages with the NAO, and Relationships with Recent Changes in the Northern Hemisphere Circulation. J. Climate, 10, 453-464.

Simmonds, I., and K. Keay, 2002: Surface Fluxes of Momentum and Mechanical Energy Over the North Pacific and North Atlantic Oceans. Meteor. Atmos. Phys., 80, 1-18.

Simmons, A. J., and B. J. Hoskins, 1978: The Life Cycles of Some Nonlinear Baroclinic Waves. J. Atmos. Sci., 35, 414-432.

Sinclair, M. R., 1994: An objective cyclone climatology for the Southern Hemisphere. Mon. Wea. Rev., 122, 2239-2256.

Sorteberg, A., and J. E. Walsh, 2008: Seasonal cyclone variability at $70 \mathrm{~N}$ and its impact on moisture transport into the Arctic. Tellus A, 60, 570-586.

Twitchel, P. F., E. A. Rasmussen, and K. L. Davidson, 1989: Polar and Arctic Lows. A. Deepak publishing, $421 \mathrm{pp}$.

Whitaker, J. S., and R. M. Dole, 1995: Organization of Storm Tracks in Zonally Varying Flows. J. Atmos. Sci., 52, 1178-1191.

White, G. H., 1982: An Observational Study of the Northern Hemisphere Extratropical Summertime General Circulation. J. Atmos. Sci., 39, 24-40.

Whittaker, L., and L. Horn, 1984: Northern Hemisphere Extratropical Cyclone Activity for Four Mid-season Months. J. Climatol., 4, 297 - 310.

Zhang, X., J. E. Walsh, J. Zhang, U. S. Bhatt, and M. Ikeda, 2004: Climatology and Interannual Variability of Arctic Cyclone Activity: 1948-2002. J. Climate, 17, 23002317. 


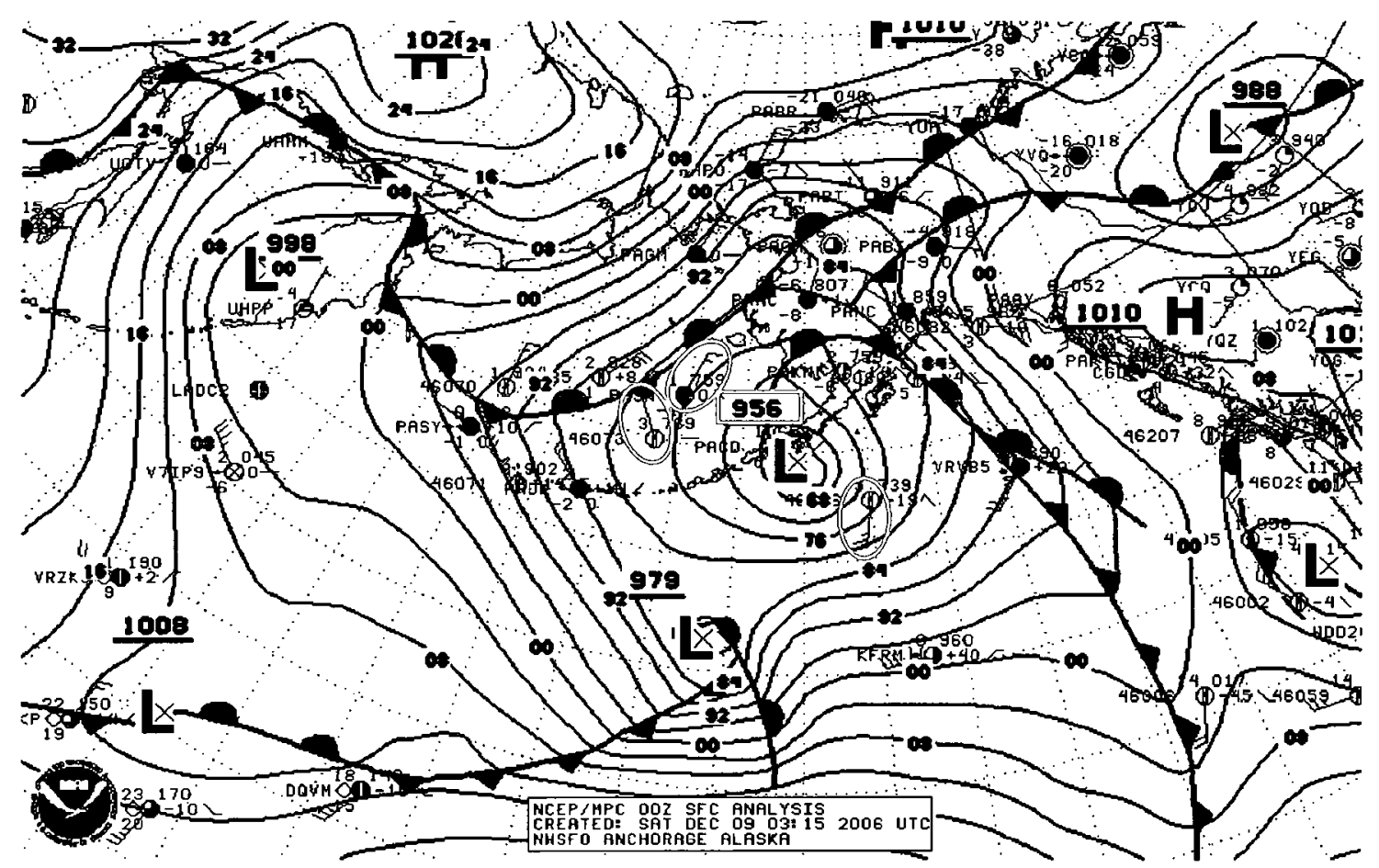

Figure 4.1 - Low over the Aleutian Islands

Mean Sea Level Pressure plot showing a low pressure system over the Aleutian Islands on 9 December, 2006. The central pressure is $956 \mathrm{mb}$ (green box). The surface winds are weak (red ellipsis), on the order of 15 knots. Figure from the NCEP/MPC 00 UTC surface analysis, provided by the NWSFO, Anchorage, Alaska. 


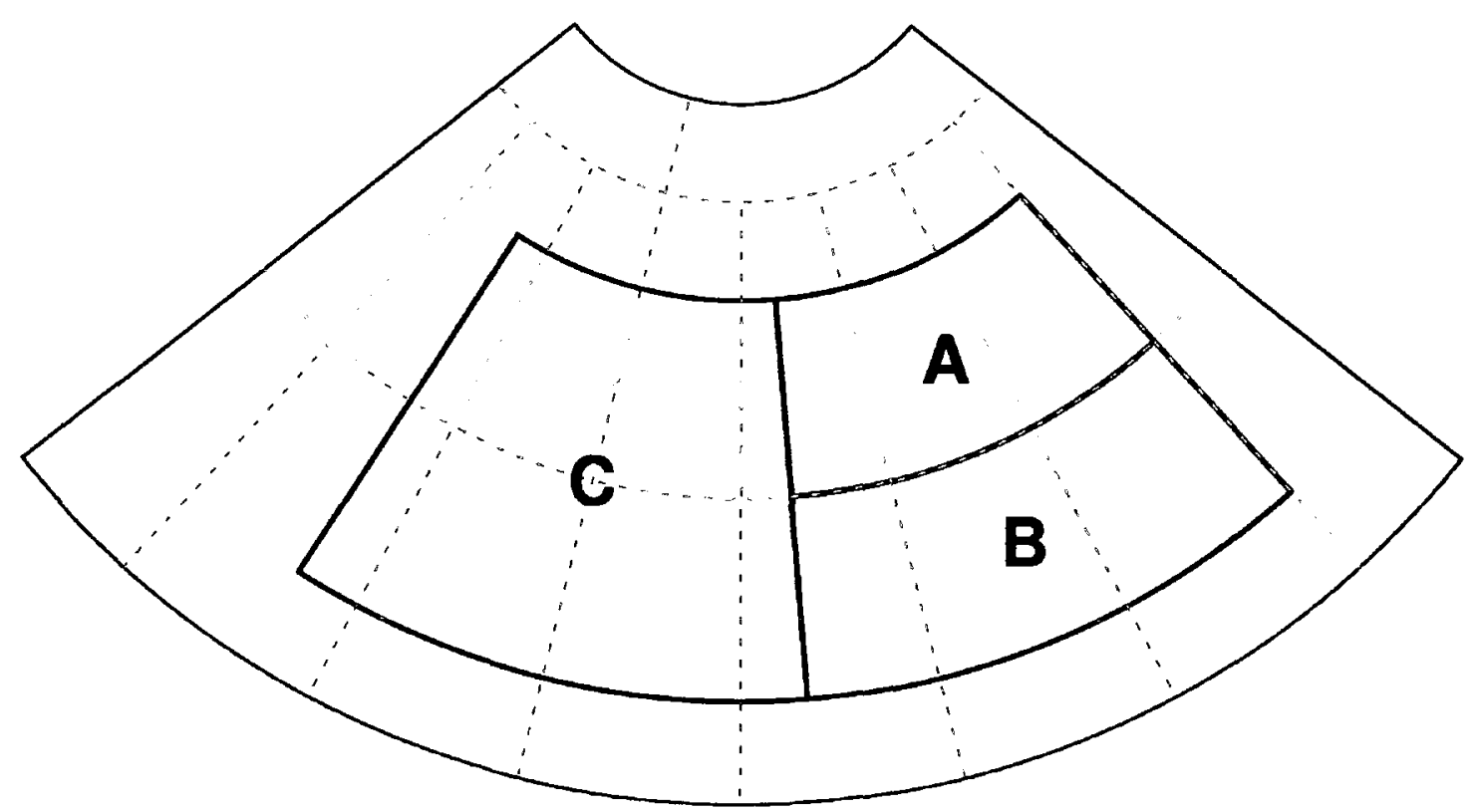

Figure 4.2 - Map of the three regions in Alaska

Three regions in Alaska studied in this paper: A - Alaska Interior/Yukon $(60 \mathrm{~N}-70 \mathrm{~N}$; $160 \mathrm{~W}-120 \mathrm{~W}) ; \mathrm{B}-$ Gulf of Alaska $(50 \mathrm{~N}-60 \mathrm{~N} ; 160 \mathrm{~W}-120 \mathrm{~W})$ and $\mathrm{C}-$ Bering Sea $(50 \mathrm{~N}-70 \mathrm{~N} ; 160 \mathrm{E}-160 \mathrm{~W})$. 


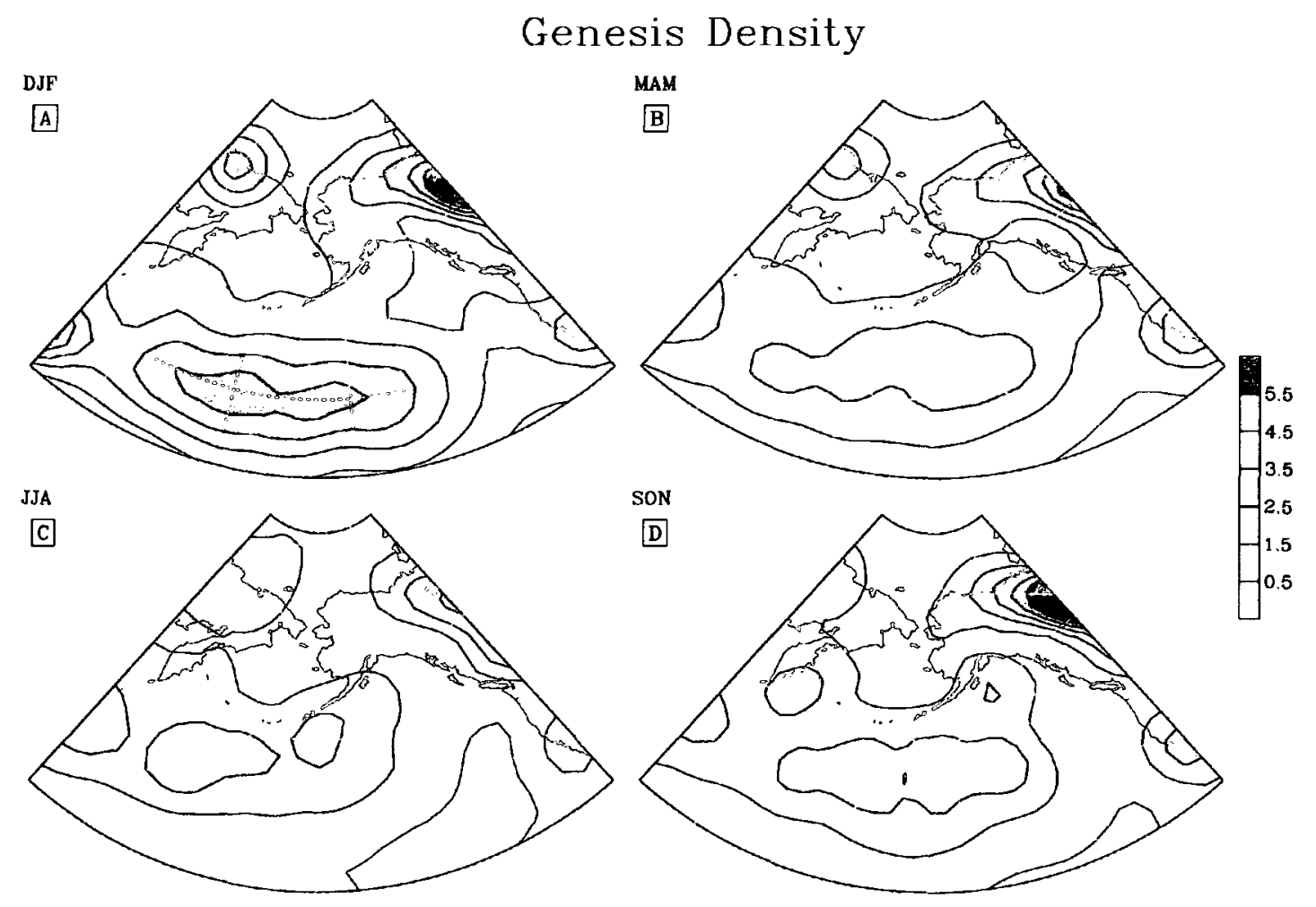

Figure 4.3 - Genesis density climatology

Genesis density climatology in the North Pacific from 1948(49) to 2008: (a) Winter (DJF); (b) Spring (MAM); (c) Summer (JJA); (d) Autumn (SON). Units: density of starting points $\left(10^{6} \mathrm{~km}^{2} \text { season }\right)^{-1}$. 


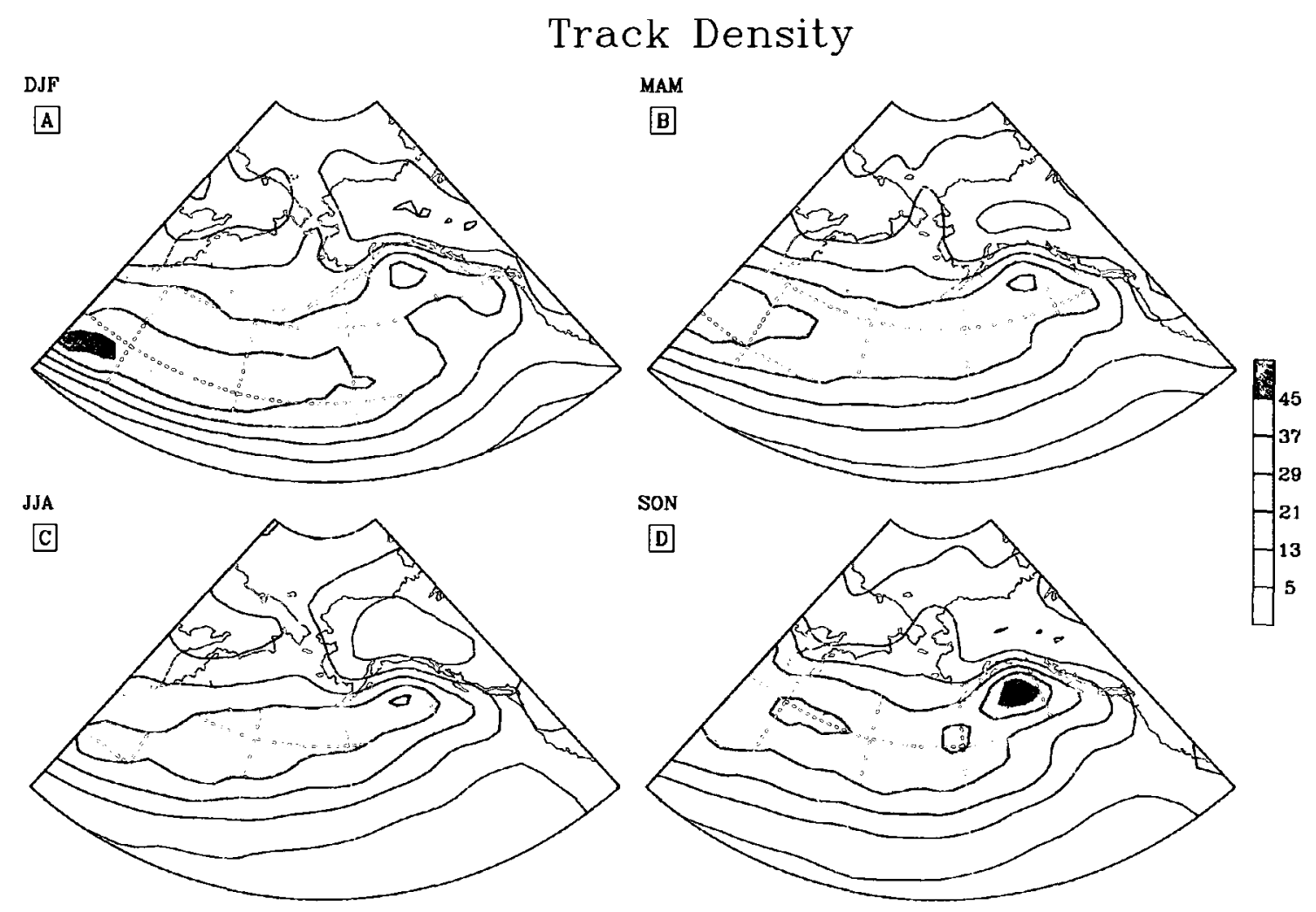

Figure 4.4 - Track density climatology

Track density climatology in the North Pacific from 1948(49) to 2008: (a) Winter (DJF); (b) Spring (MAM); (c) Summer (JJA); (d) Autumn (SON). Units: storms $\left(10^{6} \mathrm{~km}^{2}\right.$ season $)^{-1}$. 


\section{Lysis Density}
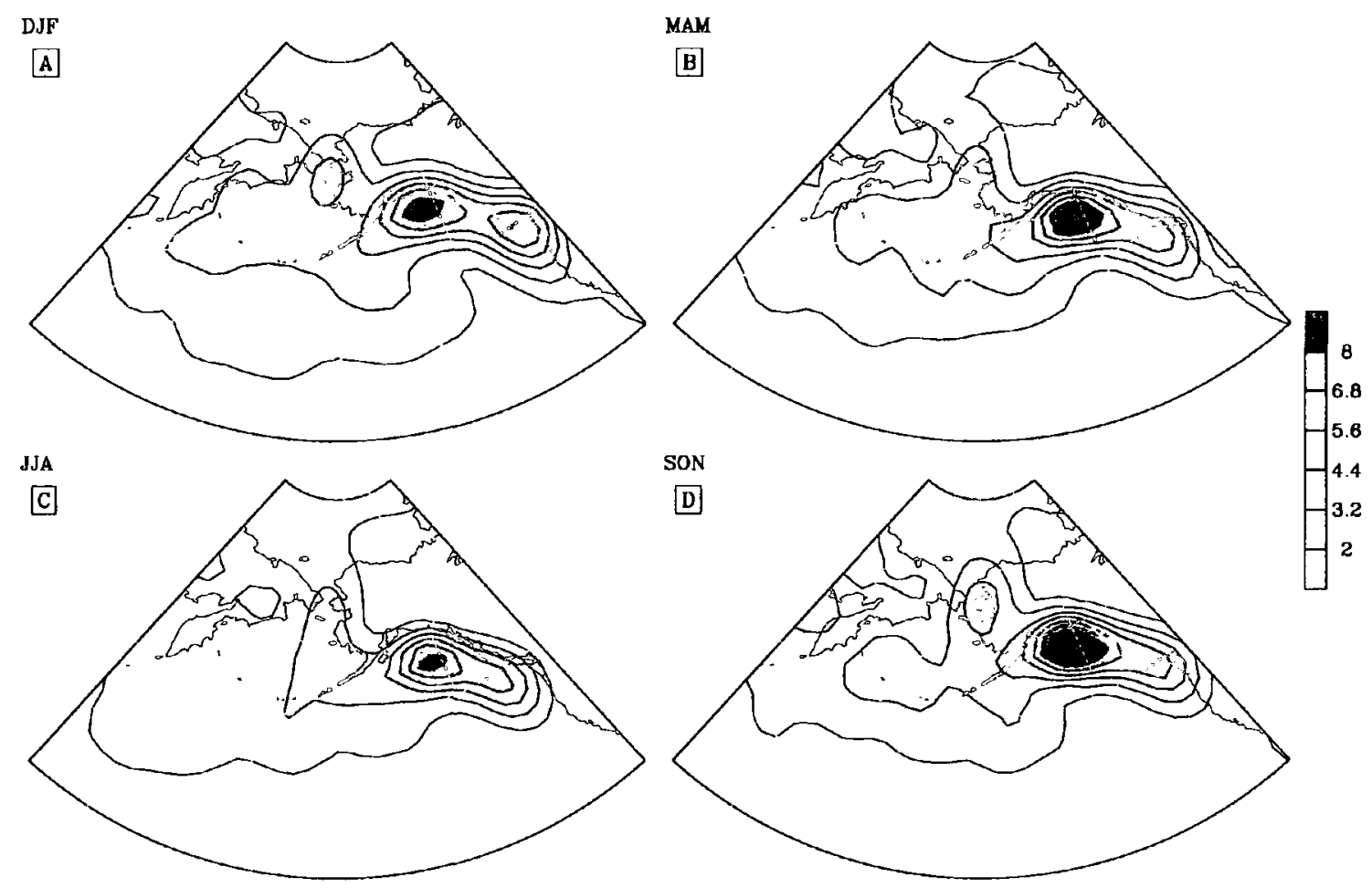

Figure 4.5 - Lysis density climatology

Lysis density climatology in the North Pacific from 1948(49) to 2008: (a) Winter (DJF); (b) Spring (MAM); (c) Summer (JJA); (d) Autumn (SON). Units: density of ending points $\left(10^{6} \mathrm{~km}^{2} \text { season }\right)^{-1}$. 


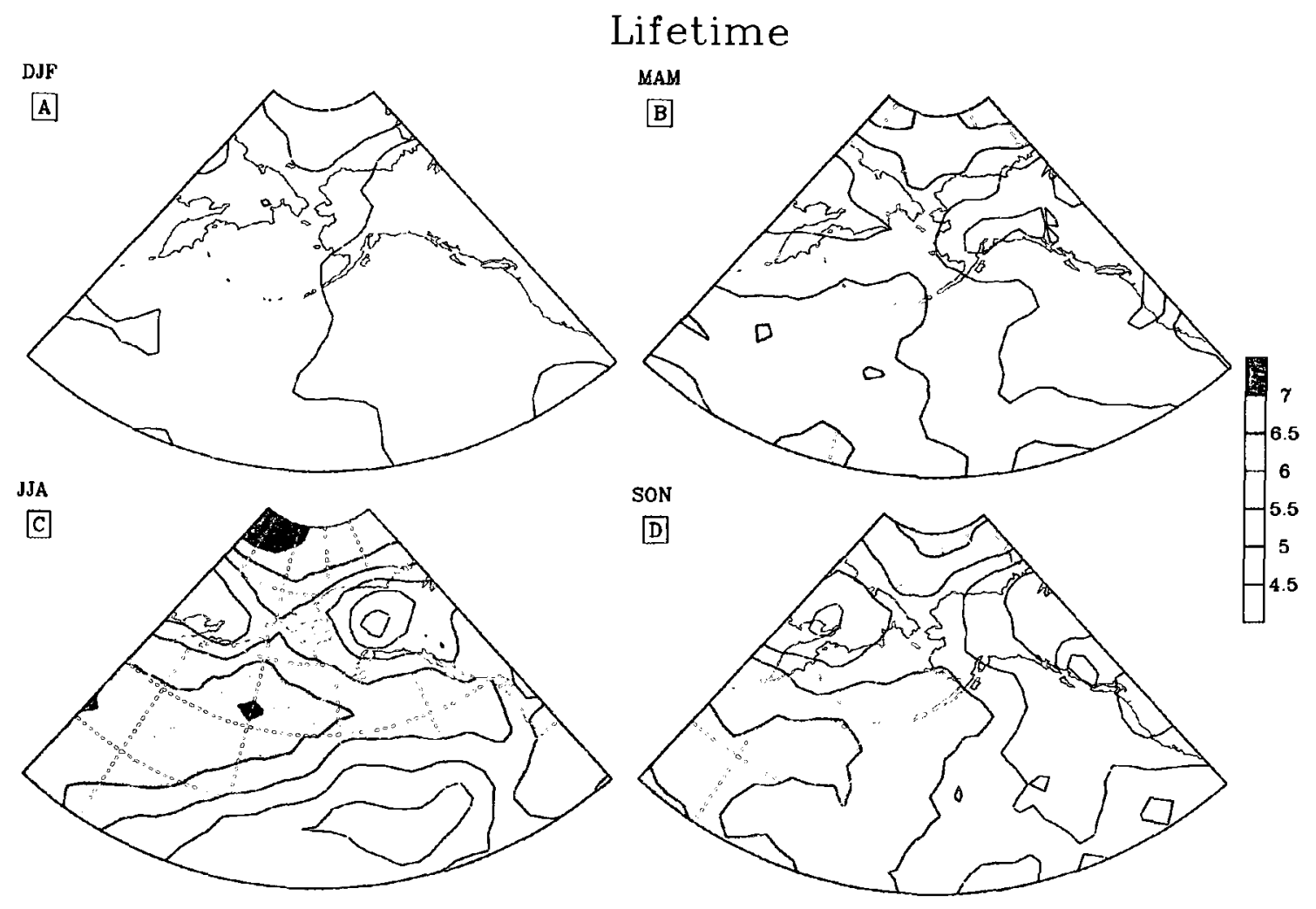

Figure 4.6 - Track lifetime climatology

Track lifetime climatology in the North Pacific from 1948(49) to 2008: (a) Winter (DJF); (b) Spring (MAM); (c) Summer (JJA); (d) Autumn (SON). Units: days. 


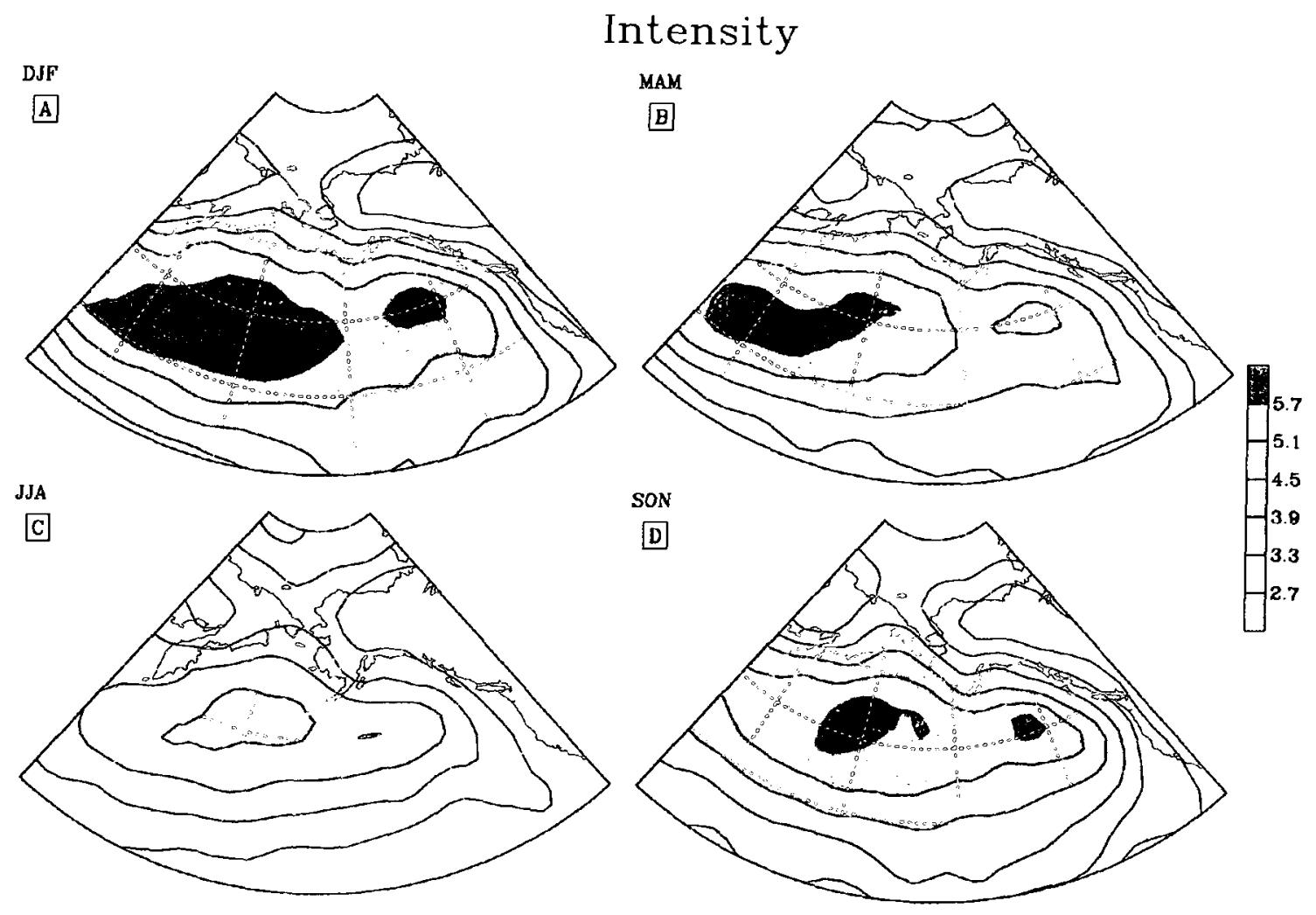

Figure 4.7 - Track intensity climatology

Track intensity climatology in the North Pacific from 1948(49) to 2008: (a) Winter (DJF); (b) Spring (MAM); (c) Summer (JJA); (d) Autumn (SON). Units: $10^{-5} \mathrm{~s}^{-1}$. 


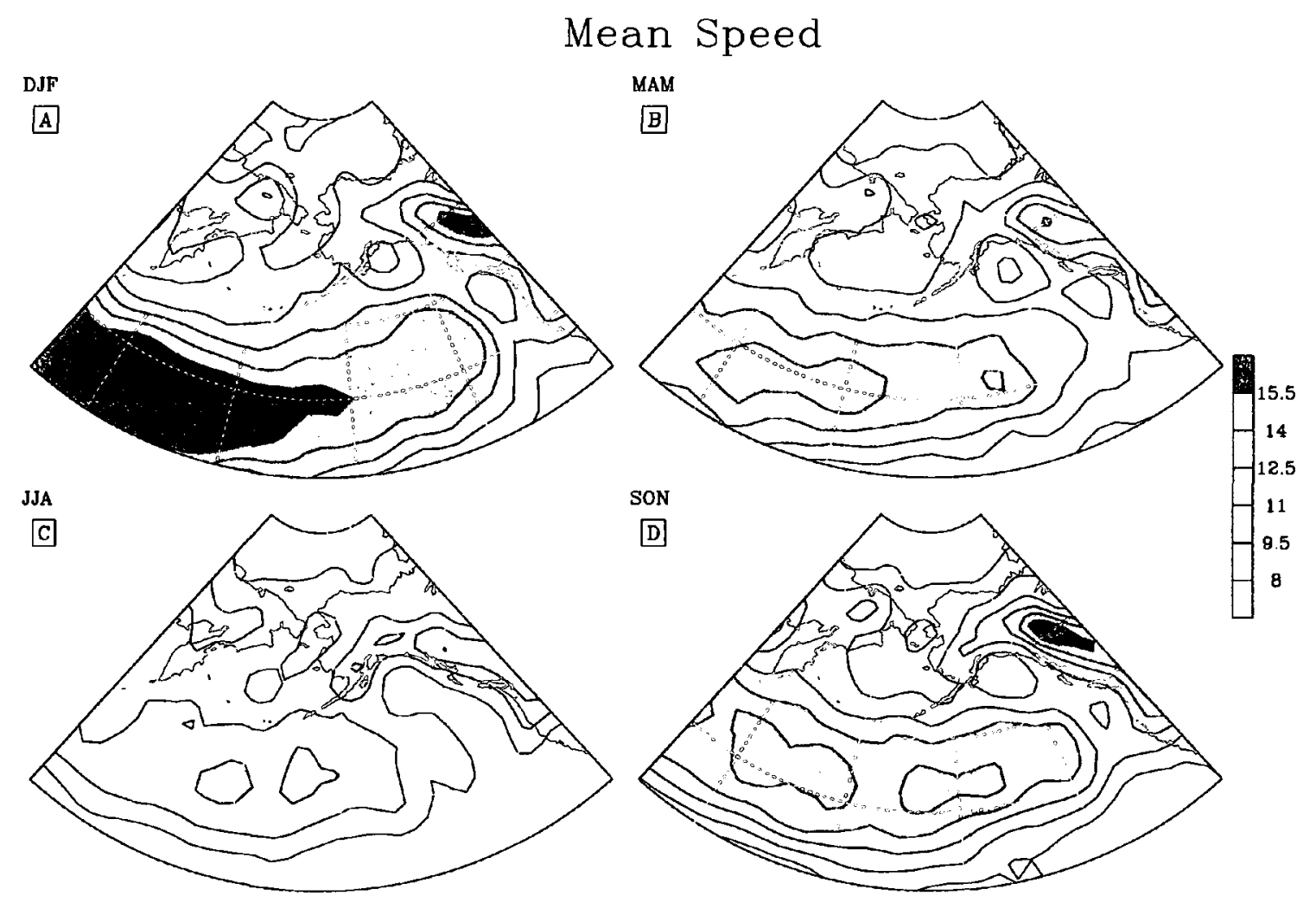

Figure 4.8 - Track mean speed climatology

Track mean speed climatology in the North Pacific from 1948(49) to 2008: (a) Winter (DJF); (b) Spring (MAM); (c) Summer (JJA); (d) Autumn (SON). Units: $\mathrm{m} \mathrm{s}^{-1}$. 


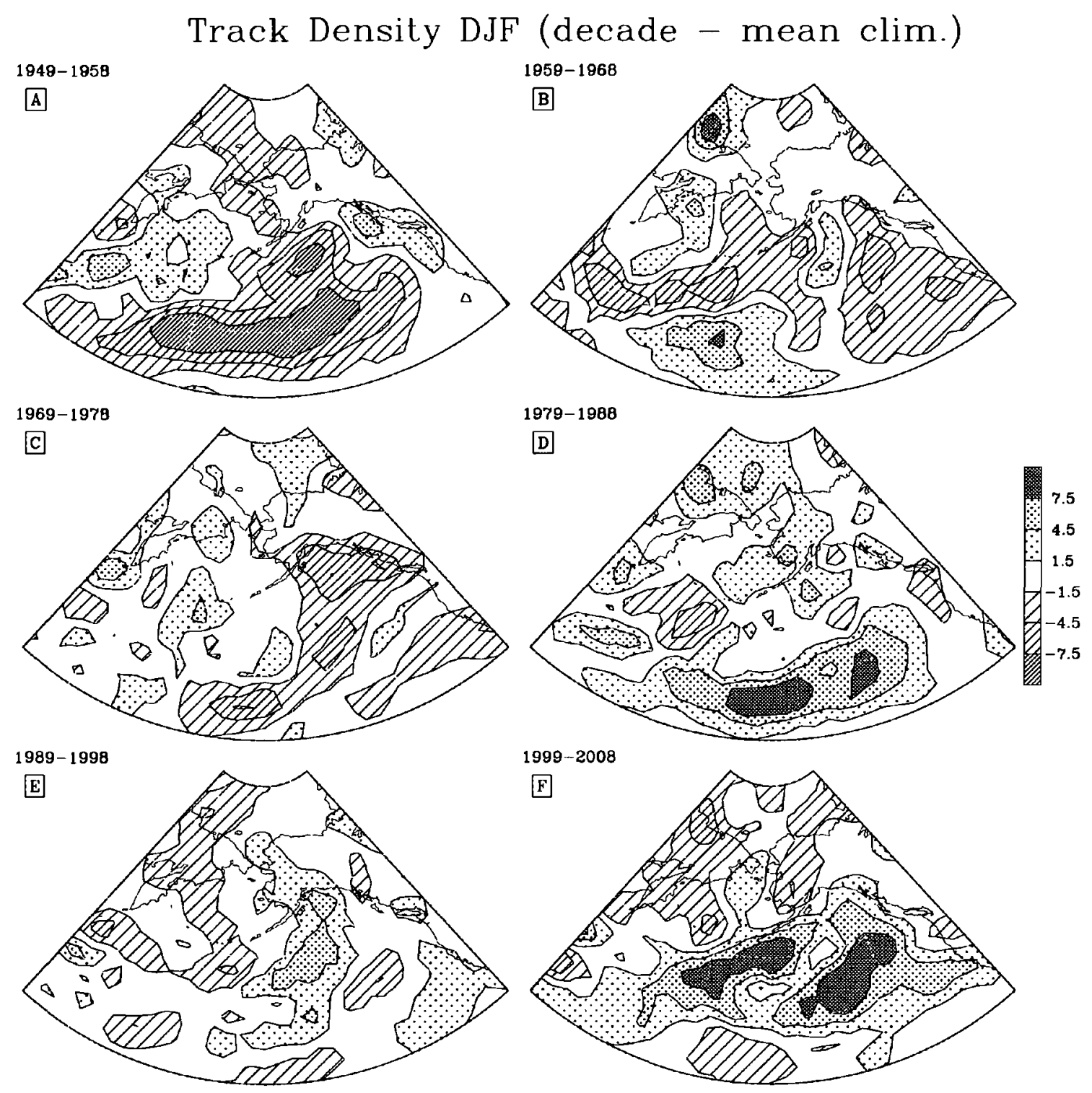

Figure 4.9 - Track density winter trend

Track density winter decadal trend represented as the decadal tdens value minus the mean climatology: (a) 1949-1958; (b) 1959-1968; (c) 1969-1978; (d) 1979-1988; (e) 1989-1998 and (f) 1999-2008. 

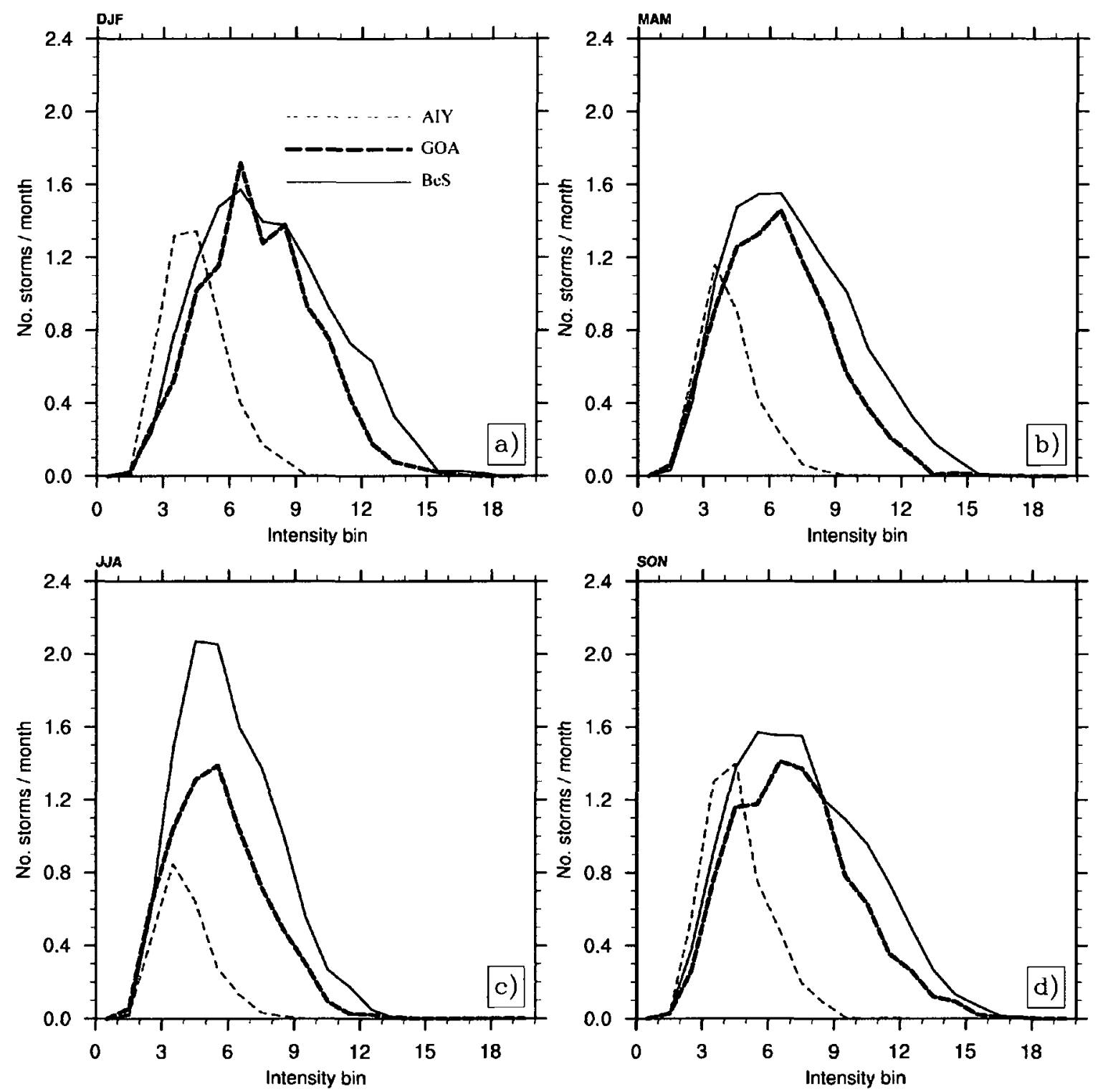

Figure 4.10 - Intensity distributions

Intensity distributions calculated from the full resolution data for AIY, GOA and BeS from 1948(9) to 2008: (a) DJF; (b) MAM; (c) JJA and (d) SON. Intensity is in units of $10^{-5} \mathrm{~s}^{-1}$; bin width is $1 \times 10^{-5} \mathrm{~s}^{-1}$. 

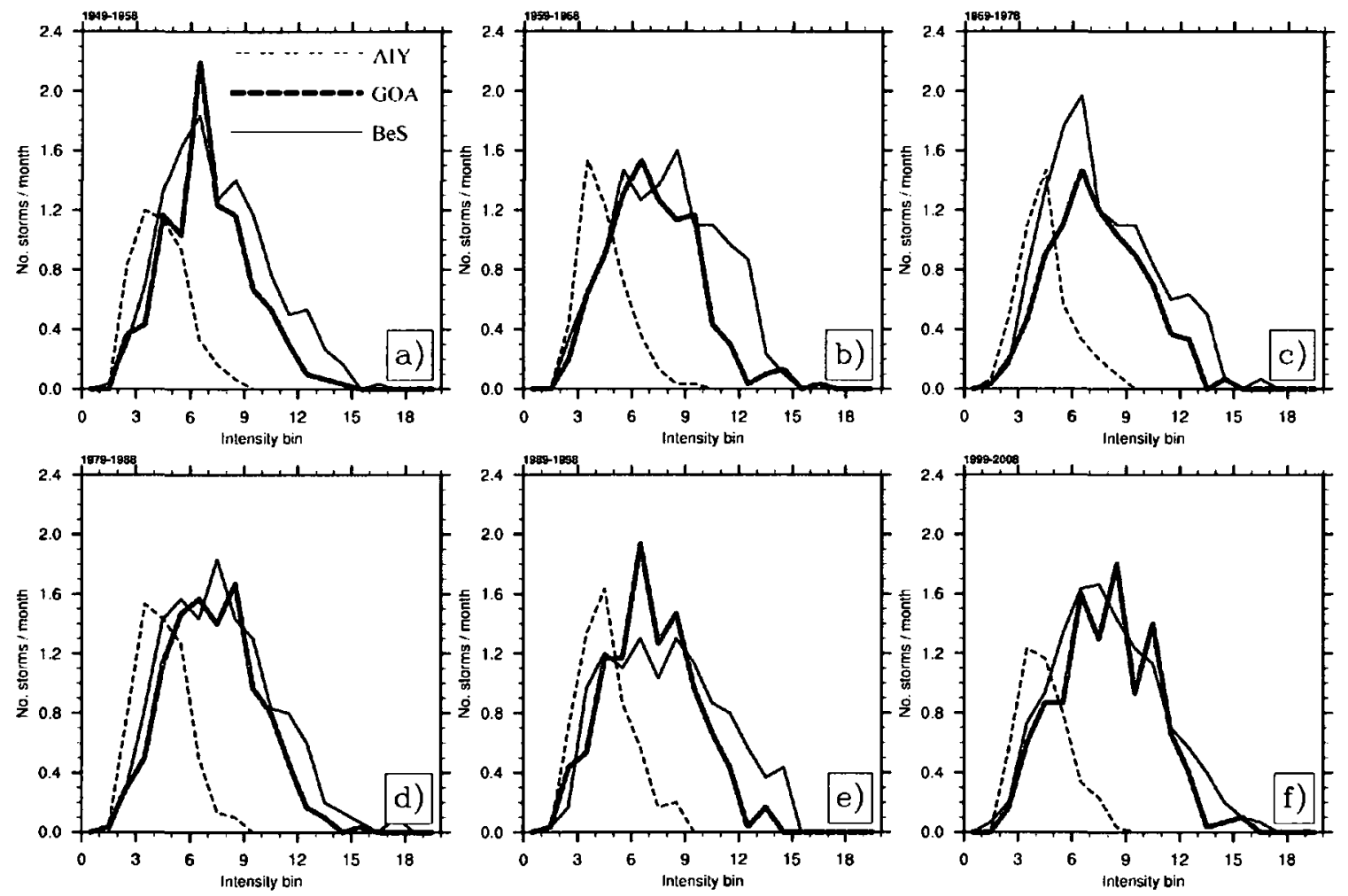

Figure 4.11 - Decadal DJF intensity distributions

The decadal DJF intensity distributions calculated from the full resolution data for AIY, GOA and BeS: (a) 1949-1958; (b) 1959-1968; (c) 1969-1978; (d) 1979-1988; (e) 19891998 and (f) 1999-2008. Intensity is in units of $10^{-5} \mathrm{~s}^{-1}$; bin width is $1 \times 10^{-5} \mathrm{~s}^{-1}$. 


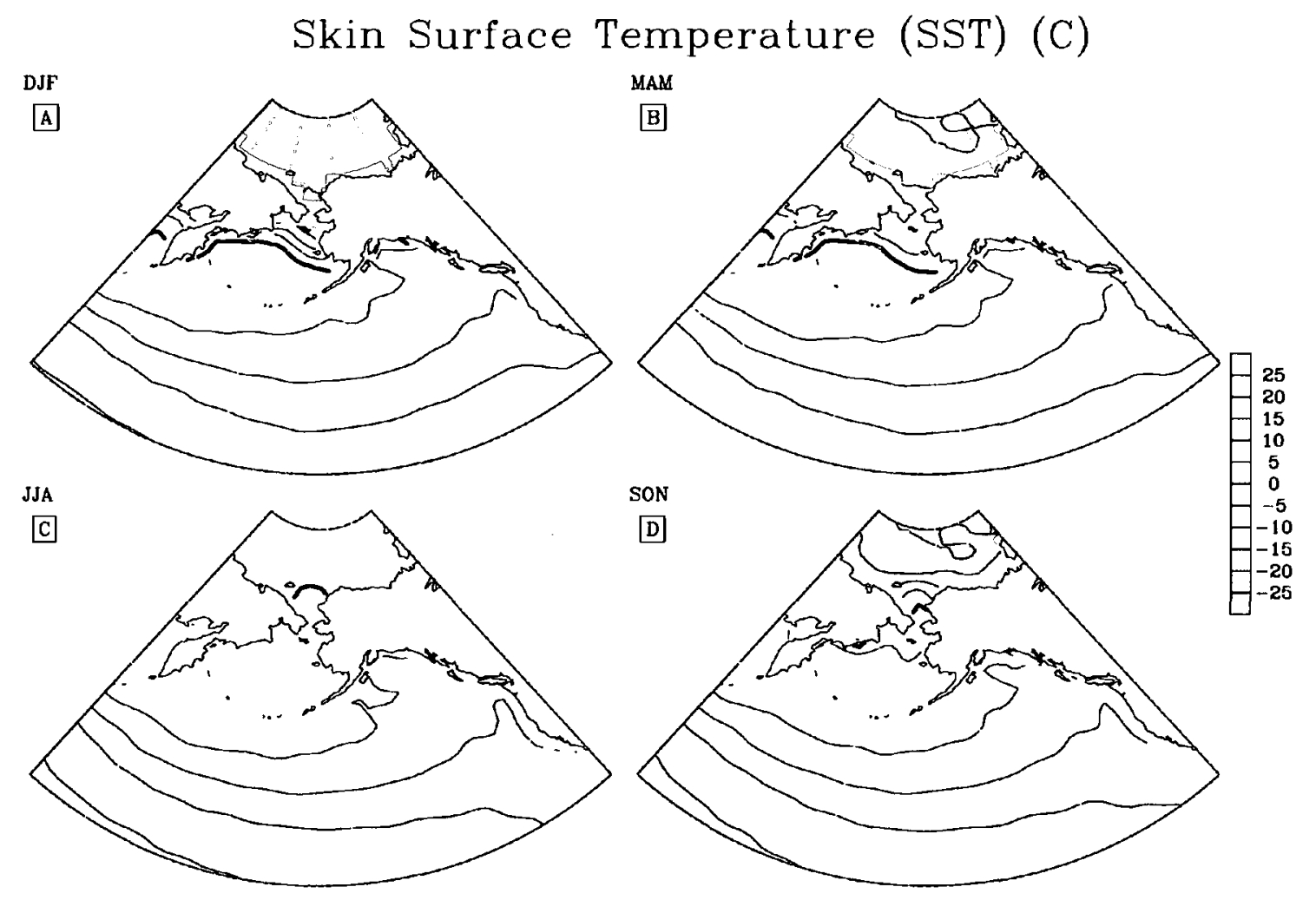

Figure 4.12 - Mean skin surface temperature in the North Pacific

Mean skin surface temperature in the North Pacific from 1948(49) to 2008 from NCEP/NCAR Reanalysis: (a) Winter (DJF); (b) Spring (MAM); (c) Summer (JJA); (d) Autumn (SON). The thick line represents the zero-degree isotherm, which provides a functional indication of the average sea-ice extent. Units: ${ }^{\circ} \mathrm{C}$. 
Genesis Density (GOA)
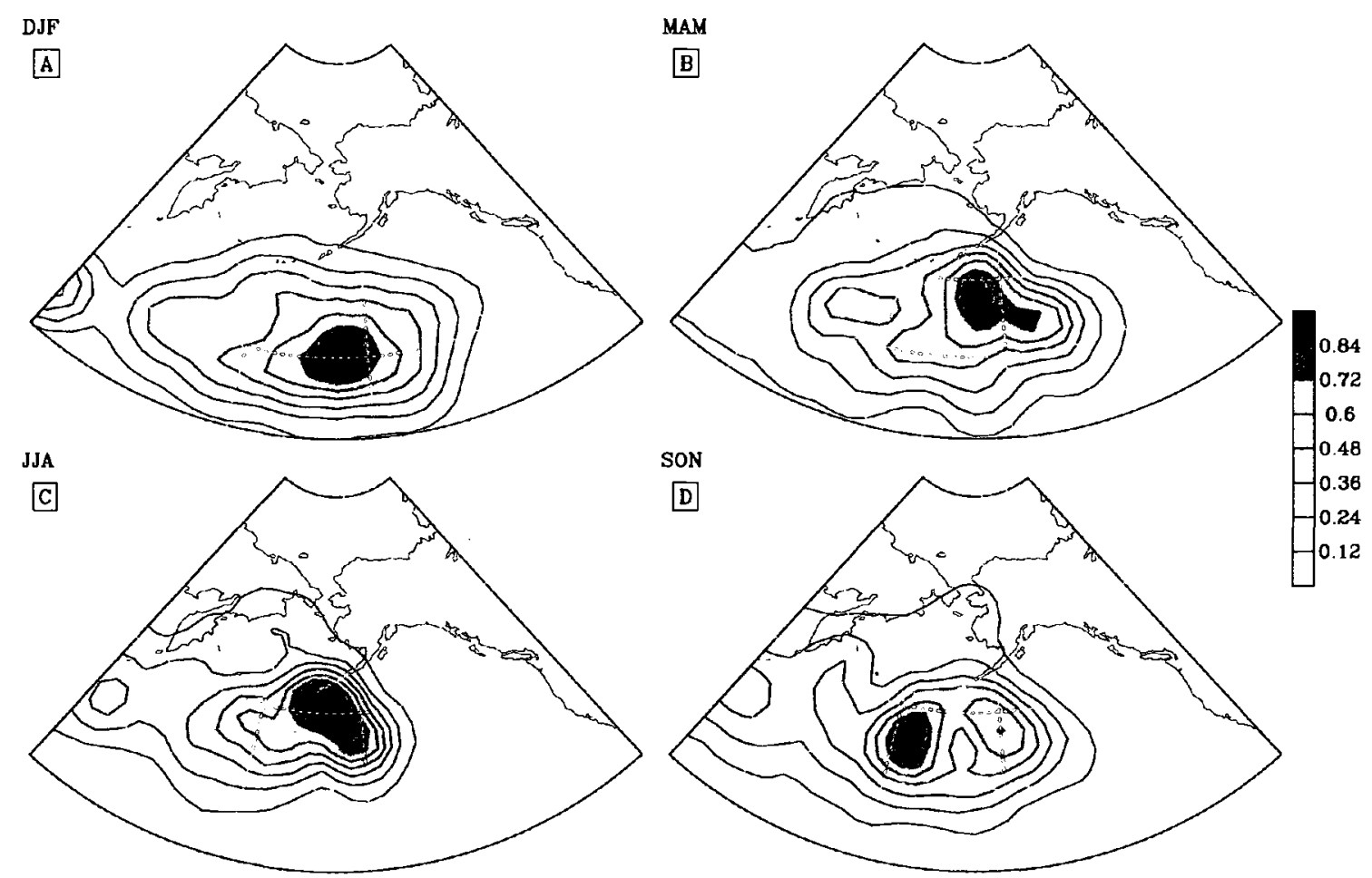

Figure 4.13 - Genesis density for end-points in GOA

Contour plots of the genesis density variable for all storms possessing end-points in the Gulf of Alaska study zone; Sampling region within a $5^{\circ}$ spherical cap and centered at $56 \mathrm{~N}$ and $147 \mathrm{~W}$; Timeframe is the entire 1948(9) to 2008 study period; plots are broken down seasonally: (a) DJF; (b) MAM; (c) JJA and (d) SON. Units: density of starting points $\left(10^{6} \mathrm{~km}^{2} \text { season }\right)^{-1}$. 


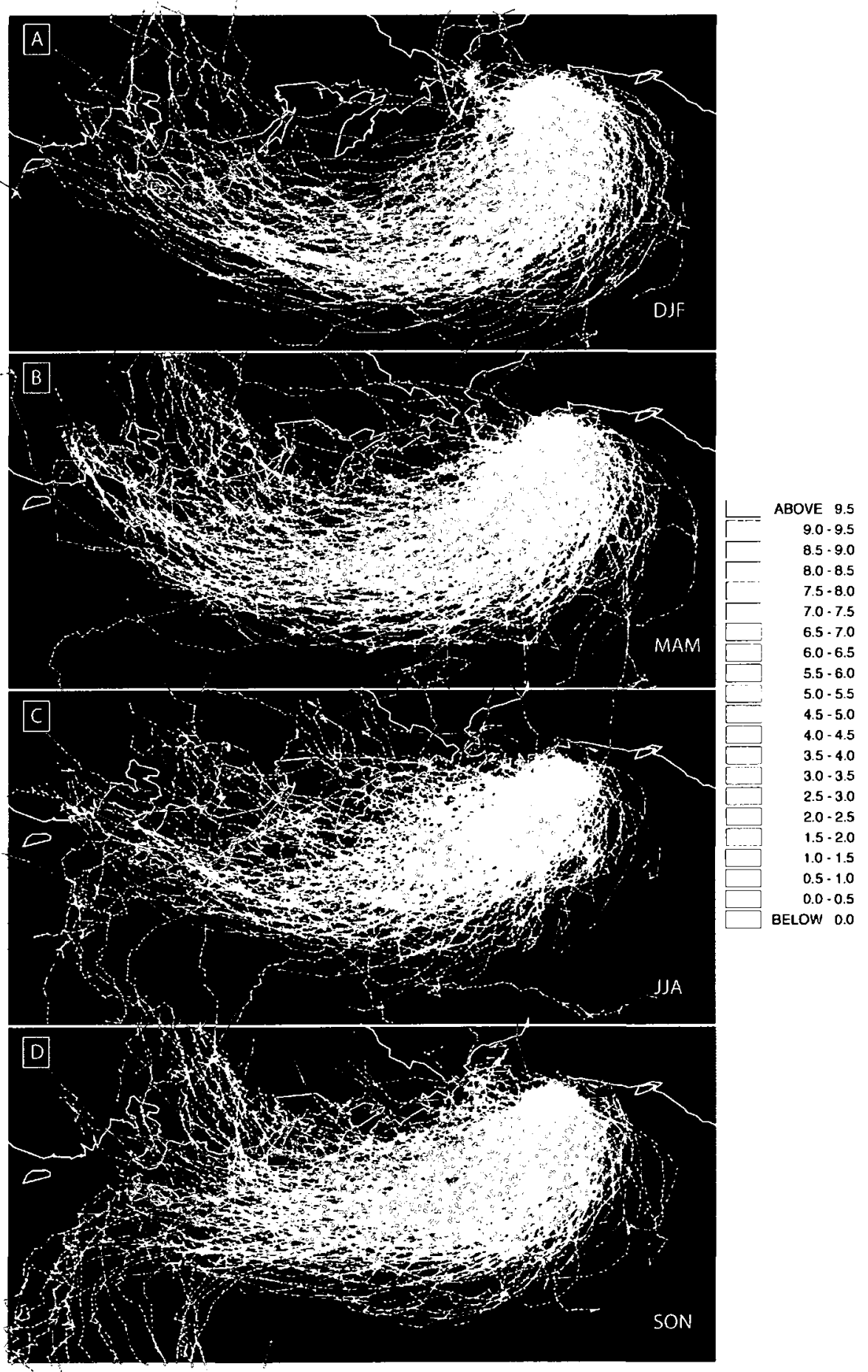

Figure 4.14 - Storms with end-points in GOA

Plots of all storms possessing end-points in the Gulf of Alaska study zone. Timeframe is the entire 1948(9) to 2008 study period; plots are broken down seasonally: (a) DJF; (b) MAM; (c) JJA and (d) SON. The color scheme represents the T42 intensities with background removed - units of $10^{-5} \mathrm{~s}^{-1}$. 
Table 4.1 - Storm track trends

Trends of two variables used in this paper: genesis density (gdens), lysis density (ldens) plus an additional metric, the number of storms entering into a region (enter). These trends are divided in three different periods: from 1948(49) to 2008; from 1948(49) to 1978 and from 1979 to 2008, and the three study regions: Alaska Interior, Gulf of Alaska and Bering Sea. Units are in $\%$ per year. Statistically significant values at the $90 \%$ s.1. are italicized, at the $95 \%$ s. 1 . are underlined and italicized, and values at the $99 \%$ level are represented in bold. Other values are not statistically significant.

\begin{tabular}{|c|c|c|c|c|c|c|c|c|c|c|c|c|}
\hline & \multicolumn{4}{|c|}{$1948(49)-2008$} & \multicolumn{4}{|c|}{$1948(49)-1978$} & \multicolumn{4}{|c|}{$1979-2008$} \\
\hline & $\mathrm{DJF}$ & MAM & JJA & SON & DJF & MAM & JJA & SON & DJF & MAM & JJA & SON \\
\hline \multicolumn{13}{|c|}{ Alaska Interior } \\
\hline gdens & 0.09 & $\underline{0.93}$ & 0.46 & 0.54 & -0.46 & 0.28 & -0.27 & 0.19 & -1.55 & 0.49 & -1.54 & -1.18 \\
\hline ldens & 0.23 & 0.49 & 0.61 & -0.01 & 0.66 & 1.47 & 1.09 & 0.69 & -1.28 & -1.92 & -1.13 & -1.34 \\
\hline enter & $\underline{-0.44}$ & 0.23 & -0.17 & 0.19 & -0.18 & -0.12 & -0.20 & 1.01 & -0.71 & 1.00 & -1.13 & -3.43 \\
\hline \multicolumn{13}{|c|}{ Gulf of Alaska } \\
\hline gdens & -0.45 & -0.14 & $\underline{-0.40}$ & -0.27 & $\underline{-1.15}$ & -0.40 & -2.09 & -1.09 & -0.86 & 0.31 & -1.98 & -1.13 \\
\hline ldens & 0.55 & $\underline{0.59}$ & 0.32 & 0.22 & 0.03 & 1.09 & $\underline{1.08}$ & -0.14 & 0.63 & -0.33 & 0.95 & 0.64 \\
\hline enter & 0.13 & 0.08 & 0.03 & 0.00 & -0.36 & -0.47 & -0.39 & -0.40 & 0.63 & 5.34 & -0.01 & 2.91 \\
\hline \multicolumn{13}{|c|}{ Bering Sea } \\
\hline gdens & 0.00 & -0.34 & 0.05 & -0.01 & -0.56 & 0.22 & 0.10 & -0.49 & 0.30 & 0.16 & 0.19 & -0.24 \\
\hline ldens & -0.08 & -0.29 & -0.09 & 0.06 & 0.10 & 0.01 & 0.05 & -0.01 & -0.04 & 0.22 & $\underline{-0.76}$ & $\underline{0.79}$ \\
\hline enter & -0.10 & -0.24 & -0.16 & -0.11 & 0.00 & -0.10 & -0.20 & -0.45 & 0.28 & -0.17 & -0.61 & -0.10 \\
\hline
\end{tabular}




\title{
Chapter 5 Sea-ice Anomalies in the Sea of Okhotsk and the Relationship with Storm Tracks in the Northern Hemisphere ${ }^{2}$
}

\begin{abstract}
Previous studies have shown that sea-ice in the Sea of Okhotsk can be affected by local storms; in turn, the resultant sea-ice changes can affect the downstream development of storm tracks in the Pacific and possibly provide an NAO damping effect in the Atlantic. In this paper, we explore these effects by tracking individual storms using the algorithm of Hodges applied to the 6-hourly horizontal winds from the NCEP reanalysis data from 1978(9) to 2007 and output from the atmospheric general circulation model (AGCM) ECHAM5 forced by sea-ice anomalies in the Sea of Okhtosk. The life cycle response of storms to sea-ice anomalies is investigated using various aspects of storm activity cyclone genesis, lysis, speed, intensity, and track density. Results show that, for positive sea-ice concentrations in the Sea of Okhotsk, there is a decrease in secondary cyclogenesis, a westward shift in cyclolysis and changes in the subtropical jet are seen in the North Pacific. In the Atlantic, a negative NAO-like pattern is observed. This pattern is confirmed by the AGCM ECHAM5 experiments driven with above normal sea-ice anomalies in the Sea of Okhotsk.
\end{abstract}

\footnotetext{
${ }^{2}$ Mesquita, M.d.S., K.I. Hodges, D.E. Atkinson, J. Bader, 2009: Sea-ice anomalies in the Sea of Okhotsk and the relationship with storm tracks in the Northern Hemisphere. Tellus $A$, submitted.
} 


\subsection{Introduction}

The relationship between extra-tropical cyclone activity and the large-scale flow has been considered in several papers (Key and Chan, 1999; Graham and Diaz, 2001; Trenberth and Hurrell, 1994). Quadrelli and Wallace (2004) showed that different largescale patterns may be described as a combination of two main modes of variability: the North Atlantic Oscillation and the Pacific North/American Index. Such patterns are termed 'teleconnections': causally linked, simultaneous or lagged correlations between temporal fluctuations in meteorological parameters at widely separated points on earth (Wallace and Gutzler, 1981). These correlations between remote points are also associated with local forcing of Rossby waves propagated downstream (Trenberth and Hurrell, 1994). These modifications of the large-scale flow can in turn be responsible for changes in storm tracks and vice-versa; for example, the influence of the NAO on storms in Europe (Ulbrich and Christoph, 1999), or the Southern Oscillation on storms in the Pacific Northwest (Allan and Komar, 2002) are well-known examples.

Franzke and Feldstein (2005) propose two alternative interpretations of lowfrequency variability: 1) that all members of the continuum can be represented by the linear combination of a small number of real physical modes or 2) that most, or perhaps all, low-frequency patterns within the continuum are real physical patterns, each with its own spatial structure and each with its own frequency of occurrence. The idea of linearly combining different teleconnections patterns was also proposed in Seierstad et al. (2007), where the authors show the linear relationship between teleconnections patterns and storminess. The authors found that the dominant factor in the North Pacific is the Pacific 
North American (PNA) pattern. In the Euro-Atlantic region, the North Atlantic Oscillation (NAO), the Scandinavian pattern, the East Atlantic pattern (EA), the EastAtlantic/Western-Russia pattern and the Polar/Eurasian pattern were significant teleconnections for that region.

Yamomoto et al. (2006) briefly summarize the "chicken and egg" discussions that now surround the linkage between the atmosphere and sea ice - what drives what. The first order view suggests that it is the atmosphere that drives the sea-ice state, as discussed by, for example, Rogers and van Loon (1979) or Partington et al. (2003). In the last 20 years, with the aid of increasingly sophisticated climate models, studies have reliably indicated that sea-ice does not passively respond to atmospheric drivers but can in fact influence the atmosphere, e.g. Honda et al. (1999). Alexander et al. (2004) explore this theme in more detail, reviewing previous work that demonstrates how the sea-ice impacts the low-levels of the local atmosphere on a fairly short-term basis, on the order of weeks, but also higher levels of the atmosphere with varying temporal lags. They also point to various hypotheses that take the temporal lag process as far as linking sea ice to decadal oscillations.

Sea ice concentration has also been shown by various scientists to have an effect on the atmosphere. For example, using a series of General Circulation Model (GCM) experiments in which southern hemisphere sea ice concentration was progressively altered from $5 \%$ to $100 \%$ open lead, Simmonds and Budd (1991) were able to demonstrate local and far field influences on a series of primary and secondary atmospheric parameters, including roughness length and upper-level winds. They go as 
far as to suggest that Antarctic sea ice influences the Southern Oscillation. An interesting point in their results is that the atmospheric response is not related linearly to the forcing of the leads - some of the largest responses occur at $80 \%$ open water, and not $100 \%$. In the Northern Hemisphere, Murray and Simmonds (1995), using perpetual January simulations to study atmospheric changes induced by reduction in Arctic sea-ice, showed a significant decrease in the speeds and intensities of cyclonic systems north of $45^{\circ} \mathrm{N}$ but little change in areal densities or in the arrangement of the major storm tracks, due to a reduction in sea ice. Johnson (1980) also studied atmospheric changes in the Northern Hemisphere due to sea ice decrease using observational data, indicating a weakened Pacific circulation and a stronger north Atlantic circulation.

A principal mechanism by which the sea-ice and the atmospheric response are linked is via the action of transient cyclones/storms. The activity of storms can have a demonstrable and almost immediate effect on sea-ice concentration and extent. Overland and Pease (1982) in fact suggest that sea-ice extent is heavily dependent on the action of storms: primarily, via their preferred pathway in a given season, and less so, via the total number of storms. The mechanism they present invokes advection as the means by which storms exert their influence: heavy ice years in the Bering Sea result from a more southerly position of the storm tracks in the Bering Sea, advecting cold air from the Alaskan and northwest North American interior regions; light ice years occur when a larger number of storms track along the west side of the Bering Sea, advecting warm air northward. 
Murray and Simmonds (1995) consider the influence of sea ice on the Northern Hemisphere cyclonic tracks and other cyclone life-cycle parameters. They found that the primary effect of sea ice removal was a monotonic but nonlinear increase in surface fluxes and lower-level temperatures in the sea ice zone. This resulted in the raising of the geopotential surfaces and a winding down of the polar vortex at $500 \mathrm{hPa}$. The authors also observed a reduction in cyclone speed and intensities due to a reduction in sea ice. They suggest that the reduction in cyclone speeds is related to a direct effect of changes in the steering flow, whereas the intensity reduction would be an indirect effect of reduced midtropospheric wind speeds via decreased baroclinicity.

The Sea of Okhotsk ('SOK' hereafter) is the southern-most marginal sea that exhibits an annual ice cover of sufficient size to exert an influence on the atmosphere. This ice cover can and is influenced by the action of storm systems, e.g. in the manner of Overland and Pease (1982), but the resultant anomalies in turn can affect the downstream development of storm tracks (Honda et al., 1999). This two-way interaction process alters local albedo and fluxes of heat and temperature, with demonstrable broader implications for Northern Hemisphere circulation.

A few studies have considered the influence of sea-ice changes over the SOK (Honda et al., 1999; Alexander et al., 2004; Yamamoto et al., 2006), either exclusively or as part of a broader study of the Northern Hemisphere, but mostly from a modeling perspective (the Honda et al. (1999) focus is on the North Pacific); Yamamoto et al. (2006) suggested that changes in sea-ice over the SOK and the Bering Sea could explain the NAO damping in later winter. 
Previous studies have shown the impact of changing sea-ice in the Northern Hemisphere polar cap in terms of the atmospheric response (Johnson, 1980; Overland and Pease, 1982; Murray and Simmonds, 1995; Deser, Walsh et al., 2000; Deser et al., 2004; Magnusdottir et al., 2004). Other studies have considered the SOK region, for example, Johnson (1980) divided the polar cap into different $30^{\circ}$ sections to understand the impact of individual zones (including the Sea of Okhotsk) - but the dataset was pre1980 and only five heavy and five light sea-ice conditions were available. Yamamoto et al. (2006) in a set of experiments in which only SOK ice anomalies were imposed showed that a damping of the NAO signal was not identified.

Murray and Simmonds (1995) have shown that the SOK region has high latent and sensible heat fluxes for different modeled sea ice conditions, however, the SOK region was not considered on its own. Murray and Simmonds (1995) also conducted a perpetual January run using a 21-wave-rhomboidally-truncated GCM to study the effects of five different open water fractions in the Arctic. This was followed by storm tracking. They found a monotonic increase in surface fluxes and lower-level temperatures in the sea-ice zone due to sea ice removal. They also found a reduction of speeds and intensity of storms. They emphasize that the response in the SOK is a local phenomenon. Their study was an important step to understanding more about the Arctic sea-ice changes and the atmospheric response, but at the time, there were some limitations to this particular study. The study of Honda et al. (1999) considered the impact of sea-ice extent anomalies in the SOK on the large-scale atmospheric circulation using an Atmosphere only GCM (AGCM) model with a 21-triangular-wave truncation and prescribed sea-ice for 
December, January and February. The authors found a stationary Rossby-wave response emanating downstream from the Okhotsk area. The findings by Honda et al. (1999) were also an important step towards showing the importance of the SOK to the large-scale flow, but again, there were limitations to the study.

Using a combination of observed SOK sea ice extent and concentrations, data from reanalyses and AGCM integrations with idealized SOK sea ice concentration, this study aims to build upon previous studies to explore, in greater detail, the following points:

a) the impact of sea-ice anomalies in the SOK on the atmospheric response to these anomalies using the observations and reanalyses.

b) establish the changes in storm tracks related to sea-ice anomalies in the SOK based on an index of sea-ice anomalies and using objective feature tracking of extra-tropical cyclones both in the reanalysis data and idealized AGCM integrations.

c) determine how the SOK anomalies are related to other teleconnections patterns based on the reanalysis and cyclone tracking.

d) determine the NAO damping signal based on a 'persistent' sea-ice concentration in the SOK region using AGCM simulations.

In this paper the word 'persistence' (as in point d) refers to similar sea-ice conditions during a season.

The next section will discuss the methodology and datasets used. In section three, the results will be discussed with respect to the storm track analysis, baroclinicity, linear 
combination of teleconnections patterns, and the ECHAM5 simulations. Section four will conclude the paper.

\subsection{Methodology and Data Sets}

Monthly sea-ice extent and concentration data for the SOK (in $\mathrm{km}^{2}$ ) were obtained from the National Snow and Ice Data Center (NSIDC) for the period 1978 to 2007. This dataset is generated from brightness temperatures derived from satellites, with radiances resolved at a grid cell size of 25 by $25 \mathrm{~km}$ (Cavalieri et al., 2008). A monthly index was created from the standardized extent and concentration datasets for December, January and February. We call these indices 'SOKe' hereafter ('Sea of Okhotsk Extent' index) and 'SOKc' ('Sea of Okhotsk Concentration' index). These will be used to produce composites of cyclone activity and other diagnostics to explore the response of the atmosphere to SOKc and SOKe anomalies.

Storms were tracked using the algorithm developed by Hodges (1994; 1995; 1996; 1999), which has yielded useful results in previous studies (e.g., Hoskins and Hodges, 2002). The tracking variables used in the algorithm were $850-\mathrm{hPa}$ relative vorticity ('RV850' hereafter) and 250-hPa ('RV250'). These fields are chosen because they are less influenced by the background flow. They also focus on the smaller-spatialscale end of the synoptic range so that many more systems are generally identified, as compared to MSLP, which focuses on the large-scale end (Hodges et al., 2003). RV850/RV250 are also related to wind speed because the relative vorticity is the vertical component of the curl of the relative velocity. RV250 was chosen to understand the 
storm track activity at higher levels. Once cyclones are identified as maxima in the relative vorticity fields the correspondence between the feature points in consecutive time steps is determined by the minimization of a cost function (See Hodges (1995) for more details). The resultant storm data was filtered to retain only those systems that last longer than 2 days and travel further than $1000 \mathrm{~km}$.

The relevant fields for the tracking were obtained from the NCEP/NCAR global reanalysis (R1) (Kalnay et al., 1996; Kistler et al., 2001) from 1978/79-2007 provided by NOAA/NWS/NCEP (NCEP Reanalysis data provided by the NOAA/OAR/ESRL PSD, Boulder, Colorado, USA, from their Web site at http://www.cdc.noaa.gov/). The tracking requires 6 hourly vorticity which is obtained from the horizontal winds. Monthly Mean Sea Level Pressure (MSLP) data are also used for regression analysis (see below). The period considered for the tracking analysis applied to the reanalysis is chosen to correspond with the SOK indices.

\subsubsection{Teleconnection Regression}

In order to assess the impact of large-scale atmospheric variability the SOKe and SOKc indices were regressed against various linear combinations of different regionallyrelevant climate variability indices. The indices consisted of: Pacific/North American (PNA), North Atlantic Oscillation (NAO), East Atlantic (EA), West Pacific (WP), North Pacific (NP), Polar/Eurasia pattern (POL) and the Northern Annular Mode (NAM). Similar linear combinations were also performed by Seierstad et al. (2007) for the relationship between teleconnections patterns and storminess. The NAM dataset was 
obtained from JISAO (Joint Institute for the Study of the Atmosphere and Ocean) on their website at: http://jisao.washington.edu/analyses0302/slpanompc.ascii. The NP index data was downloaded from UCAR (University Corporation for Atmospheric Research) at: http://www.cgd.ucar.edu/cas/jhurrell/indices.data.html\#npmon. The other indices were obtained from the NOAA Climate Prediction Center from their website at: $\mathrm{ftp} / / / \mathrm{ftp} . c p c . n c e p . n o a a . g o v / w d 52 \mathrm{dg} / \mathrm{data} /$ indices/tele_index.nh. The data used were from 1979 to 2007 . The reader is referred to the aforementioned websites for more details on the indices.

The combinations were also tested with and without the intercept in the multiple regression equations, which may improve the significance of the combinations. A linear trend term was also tested, representing the years, as in Seierstad et al. (2007). The predictive skill of each regression model was quantified using Akaike's Information Criterion (AIC), which is a measure of the goodness of fit of a statistical model (Akaike, 1974); AIC is here used to select the 'best' predictive regression models. The three models with best AIC predictive skills were retained for further analysis. The smallest value of AIC is preferred.

\subsubsection{Model and Experiments}

The atmospheric model used in this study is the AGCM ECHAM5 (Roeckner et al., 2006), described in full detail in a technical report (Roeckner et al., 2003). This model has previously been used for sea-ice experiments (see e.g., Seierstad and Bader (2008)). 
The atmospheric model is run at $\mathrm{T} 63\left(\sim 1.875^{\circ} \times 1.85^{\circ}\right)$ horizontal resolution with 31 vertical levels. Two sets of ensembles, each consisting of 30 experiments, were conducted. Each experimental member only differs in the initial conditions, which were chosen from a separate control integration.

For computational economy, each experiment was run for 4 months starting from first of December until the end of March. The ECHAM5 model is forced by the climatological annual cycle of global sea surface temperatures and sea ice concentrations except in the SOK. In the SOK (see Fig. 5.9) the sea ice concentrations have been set to zero for one ensemble and to $100 \%$ for the other. All other external forcings (e.g.: greenhouse gases, solar radiation) are the same for all integrations. The only difference between the two ensembles is the sea-ice concentration in the SOK. Figure 5.10 shows the fluxes of the sensible plus latent heat anomalies.

\subsubsection{The Compositing Method}

The analysis in this paper use the compositing method described in Bengtsson et al. (2006). The benefit of using the weighting in the composite method is that more of the data can be used as opposed to applying a straightforward threshold, this can be important if the data period is relatively short.

The compositing works by constructing a set of weights based on some montonic function, in this case the tanh function. These weights are incorporated into the kernel estimators - they weight each track point according to what time period it lies (i.e. month). Kernel estimators associate a kernel function (probability density function) to 
each data point. This spreads the influence of a data point over some region called the support region (Hodges, 1996). These estimators are applied on the sphere to prevent the kind of biases one gets with histogram methods on a projection (Hodges, 1996). Performing this for positive and negative indices separately produces statistics for the positive and negative phases.

The estimators used for the composite statistics are (Bengtsson et al., 2006):

$$
\begin{aligned}
\hat{f}(\mathbf{X})=\frac{\sum_{i=1}^{n} w_{i} K\left(\mathbf{X}_{i} \cdot \mathbf{X}\right)}{\sum_{i=1}^{n} w_{i}} \\
\hat{\mathbf{Y}}(\mathbf{X})=\frac{\sum_{i=1}^{n} w_{i} \mathbf{Y}_{i} K\left(\mathbf{X}_{i} \cdot \mathbf{X}\right)}{\sum_{i=1}^{n} w_{i} K\left(\mathbf{X}_{i} \cdot \mathbf{X}\right)}
\end{aligned}
$$


Equations 5.1 and 5.2 represent the density estimation and the mean attribute estimation, respectively. $\mathbf{X}_{\mathrm{i}}$ is the unit vector to data point $i$ on the unit sphere. $\mathbf{Y}_{\mathbf{i}}$ represents the data point $i$ value (scalar or vector). The effective number of data points is given by:

$$
N_{e}=\frac{\sum_{i=1}^{n} w_{i}}{\sum_{I=1}^{M} w_{I}}
$$

where the additional weights are chosen as $w_{i}(I)=\tanh \left( \pm 1.5 W_{l}\right)$, if $\pm 1.5 W_{l}>0 ; 0$ otherwise. The weight is represented by $w_{i}$, dependent on the year and month for data point $i$. $W_{I}$ represents the monthly index value for month $I . K(\cdot)$ is the spherical kernel, $n$ is the number of data points, and $M$ is the total number of months.

As in Bengtsson et al. (2006), the tanh function, which has values between -1 and +1 , is used. The scaling of the tanh argument is chosen to give weights close to 1 at 1 standard deviation for both positive and negative indices. So for the positive phase the weights for negative indices are set to zero and the positive indices are set to the tanh value, this is reversed for the negative phase.

Once the positive and negative phase statistics have been computed they are differenced to highlight the changes between the two phases. 


\subsubsection{The Significance Tests}

The methodology used to provide an indication of the significance for the difference plots are computed using a permutation Monte Carlo approach. Hodges (2008) describes the methodology in detail. The advantage of working with the Hodges' approach is that it "...improves the efficiency with which statistical estimates of the distribution and mean attributes of dynamical weather systems, such as extratropical cyclones and tropical easterly waves, are derived from ensembles of the system trajectories using spherical kernel estimators", as described in Hodges (2008) page 1758.

In this methodology, Monte Carlo methods are used to resample the data repeatedly to produce new statistical estimates for each of the new samples - the sampling distribution can then be determined from these. The method uses a permutation approach by pooling two datasets into one and then resampling without replacement. This produces two new-paired samples of the same size as the original data samples. Hodges (2008) also describes that for track datasets, the resampling samples the tracks as "complete entitites". This is repeated a large number of times - and from these new paired samples, the track statistics and their differences are then computed.

The p-values are obtained by determining the probability that a more extreme value of the test statistic (difference) is possible than that obtained form the original pair of samples. A two-tailed test is used. No assumptions about the sampling distribution are made and since the sampling distribution may be asymmetric, a p-value estimate given by Davidson and MacKinnon (2003) and described in Hodges (2008) is used. This 
approach has been applied in other storm track studies (Bengtsson et al., 2006). The pvalues are plotted for values below $5 \%$.

\subsection{Results}

\subsubsection{The SOK Indices}

In Figure 5.1a the mean SOK sea-ice concentration is shown and the SOKc anomaly index timeseries in Figure 5.1b. The sea-ice extent and concentration in the SOK are highly correlated (not shown), and even though both indices were tested in this study, only results using SOKc were retained for further analysis. On average, the SOKc index was positive from 1979 to 1987 (except 1981 and 1984), from 1993 to 1995 and from about 1999 to 2003 (except 2000) and it was negative otherwise. In spite of this and the slight decline in the early 1980s, no particular long-term trend is evident when considering the entire timeseries. The year 1996 was the record minimum sea-ice extent (not shown) in the Sea of Okthosk for the months of January and December, in agreement with Toyota et al. (1999) page 119, which observed that 'Since the data became available in 1971, the sea ice extent in the Okhotsk Sea in 1996 was recordbreakingly small...'

\subsubsection{Extra-tropical Storm Tracks and the SOKc Index}

The statistics of the storm track patterns (Figure 5.2) during the positive and negative phases of SOK were determined based on the compositing method in Bengtsson et al. (2006). These tracking statistics are calculated using spherical kernel estimators, 
described in Hodges (1996) and the influence of the SOKc index is introduced into the statistics by introducing additional weights computed from the index as described by Bengtsson et al. (2006). The response to SOK was calculated by producing composite statistics for the positive and negative phases using the SOKc index for the DJF period from the NCEP reanalysis data for 1978-2007. Figure 5.2 shows the difference between the positive and negative (SOKc+ minus SOKc-) composites of SOK for four track variables: a) genesis density (gdens) - density of track starting points per $\left(10^{6} \mathrm{~km}^{2}\right.$ season $)^{-1}$; b) track density $($ tdens $)$ - number of storm tracks through a region per $\left(10^{6} \mathrm{~km}^{2}\right.$ season $)^{-1}$; c) lysis density (ldens) - density of ending track points $\left(10^{6} \mathrm{~km}^{2} \text { season }\right)^{-1}$; and d) mean intensity (mint) - mean strength of the vorticies in units of $10^{-5} \mathrm{~s}^{-1}$. Here, results will be focused mainly on the Pacific and Atlantic changes. The thick black lines show regions where the p-values are less than $5 \%$.

The gdens plot (Figure 5.2a) shows more storms being formed in parts of the Bering Sea, Kamchatka and southern Alaska when SOKc is positive, consistent with Overland and Pease (1982). They could represent generation of storms due to local baroclinicity, since higher sea-ice in the Sea of Okhtosk could lead to higher north-south gradient of temperature in the Kamchatka area. In the Atlantic, more storms are observed to generate over the western approaches of the United Kingdom, these are also most likely due to secondary generation. More storms are also seen to generate east of the United States.

Negative values are found over the SOK, indicating that more sea-ice in that region helps to cut off the latent heat source for storms to be generated there. Another 
striking feature is the reduction in the storms over the main track in the Pacific, the region of secondary cyclogenesis (Hoskins and Hodges, 2002). Changes in gdens over the Southern Rockies are also indicative of changes in the subtropical jet: a decrease of gdens in the southern Rockies and an increase to the North. The gdens decrease in the Atlantic is mostly along the east coast of North America (Nova Scotia) and through the Icelandic region towards Scandinavia. The reduction over Nova Scotia is mirrored by an increase further south over Cape Hatteras which together with the increased secondary cyclogenesis downstream may indicate a more southerly storm track. There is also an enhancement through the Mediterranean. The Atlantic results have some similarity with a negative NAO distribution (see later).

The tdens plot (Figure 5.2b) show results that reflect the differences in gdens, except for a stronger negative signal in the Gulf of Alaska and western Pacific - a suppression of storms in that area. This looks like an equatorward shift at the end of the Pacific storm track, though this is less apparent in the regression of the $500-\mathrm{hPa}$ geopotential (Figure 5.3b, d, f) and MSLP (Figure 5.3a, c, e).

In the Atlantic, there seems to be an equatorward shift in the storm track with more storms into the UK and through the Mediterranean (similar to a weak NAO). This agrees with the regressed 500-hPa geopotential (Figure 5.3b, d, f) and MSLP (Figure 5.3a, c, e). This is also confirmed by the Eady growth rate composite plots (Figure 5.4). Figure 5.4a shows the 850-hPa Eady growth rate for $\mathrm{SOKc}+$ (red) minus SOKc- (blue). The contour lines represent TDENS for SOKc+ (solid) minus SOKc- (stipled). For comparison, Figure 5.4b shows the 500-hPa geopotential height of SOKc + minus SOKc-. 
The pattern observed in the figures has some similarities with the negative NAO pattern. These negative-NAO-like changes could be related to the NAO damping due to changes in the sea-ice concentration over the SOK, as suggested by Yamamoto et al. (2006). The authors showed that changes in the North Pacific and North America are linked to changes in the sea-ice variability of the SOK through the formation of a stationary Rossby wave train. They also suggested that a positive phase of the Northern Hemisphere ice seesaw (sea ice variability pattern with one polarity in the Bering and Labrador Seas and the other in the Okhotsk and Greenland-Barents Seas) - dominant in midwinter - appears as a response to a positive NAO and anticyclonic anomalies over the North Pacific in early winter. In turn, the midwinter positive sea-ice seesaw damps the positive NAO signal. In late winter, a negative phase of the NAO is observed - damped by the sea-ice seesaw in the North Pacific: reflected as a stationary Rossby wave excited over the SOK.

A striking difference is seen in the ldens plot (Figure 5.2c) in the Pacific. The west Pacific shows more storms dying there when SOKc is positive. It is important to emphasize that SOKc could also be a response to cold temperatures and sea-ice conditions over Eurasia and Siberia which could also be affecting the North Pacific (Orsolini and Kvamstø, 2009). Hence, cold temperatures as seen in the positive phase of SOKc could suppress the storm tracks, making them die earlier, before reaching the Gulf of Alaska ('GOA' hereafter). GOA is known to be the region where most storms die in the North Pacific (Simmonds and Keay, 2002, Hoskins and Hodges, 2002) and trends over GOA are not explained by the local changes of SST (Mesquita et al., 2009). For 
positive SOKc, storms die before reaching GOA: either in the west Pacific or over the Aleutians. Storms that are generated over the Aleutians/Bering Sea end up dying over the Yukon/British Columbia area.

In the Atlantic, we see a coherent pattern in the Idens case: storms die more frequently over the UK and central Europe and less in the region between southern Greenland, Scandinavia and eastern Europe - patterns very similar to the negative NAO pattern.

The mean intensity (Figure 5.2d) pattern shows less intense storms over and south of the Kamchatka area, with more intense systems found close to the Bering Strait and over the Aleutians. In the Atlantic, a shift in the pattern is observed; in this case more intense systems in the south and less intense in the North.

\subsubsection{Baroclinicity}

As pointed out by Weber and Névir (2007), a number of diagnostic parameters can be used for analyzing atmospheric structures (e.g.: Ertel's potential vorticity, Eady growth rate, the Dynamic State Index). In order to study the baroclinicity for positive and negative years of SOKc, weighted composite analysis were computed (Figure 5.4). The measure of baroclinicity used was the Eady growth rate:

$$
\sigma_{B I}=0.31 \frac{f}{N}\left|\frac{\partial \vec{v}}{\partial z}\right|
$$


where $f$ is the Coriolis parameter, $N$ is the Brunt-Väisällä frequency, $z$ is the vertical distance and $v$ is the horizontal wind vector. The Eady growth rate is a means to achieve a better understanding of the process of baroclinicity. It can be used to localize baroclinic zones: this is important since regions of high baroclinicity over the mean Northern Hemisphere winter conditions correlate well with regions of high eddy activity (Chang and Orlanski, 1993).

The calculation was based on the monthly NCEP/NCAR Reanalysis data (temperature, geopotential height and horizontal winds) at the $850-\mathrm{hPa}$ level from 1978(9) to 2007(8). Even though monthly values were used, the Eady growth rate calculation was conducted only to provide an indication and conclusions are not based on it. Figure 5.4a shows the weighted composite of the positive (in red) minus negative (in blue) phases threshold of SOKc. Overlayed to the plot is the positive (solid lines) minus negative (stipled lines) TDENS (as in Fig. 5.2). The figure shows good agreement between the Eady growth rate and TDENS differences especially in the main regions considered in this paper: the Atlantic and the Pacific. This also means that there is a strong association for the positive and negative phases of SOKc during winter with the baroclinic zones indicated by the Eady growth rate.

Even though the agreement is not for all regions, it is important to note that Chang and Orlanski (1993) argue that in spite of the fact the Eady growth rate can localize baroclinic zones, it does not relate to the location of the eddies, which are found downstream of the source baroclinic region. Chang and Orlanski (1993) have also shown that the ageostrophic geopotential fluxes by an upstream eddy acts as a trigger for the 
development of downstream cyclones. Thus, the ageostrophic fluxes contribute to the growth and decay rates in the nonlinear life cycles of individual cyclones, since they act as a source of energy for the growth of the next downstream wave (Orlanski and Katzfey, 1991).

The geopotential height at the $500 \mathrm{hPa}$ level was also plotted using the same weighted composite technique. It is shown in Figure 5.4b. The composites are also in good agreement with Figure 5.4a, especially over the Atlantic.

\subsubsection{Linear Combination with Teleconnection Patterns}

The linear regression of the SOK index onto the MSLP and HGT500 is shown in Figure 5.3. We have easterly winds from the Kamchatka/Russia regions towards the SOK (mainly in December and January), indicating the relationship between the atmospheric circulation and sea-ice, in this case, favoring the sea-ice extent. The overall pattern resembles mostly the East Atlantic pattern (EA), the North Atlantic Oscillation and the West Pacific pattern (Figure 5.8a-c), or a hybrid pattern between them (See Franzke et al. (2005)): MSLP decrease over the North Pacific and North Atlantic leading to an increase in the number of storm tracks in these regions. The similarity of the regression patterns to well known teleconnections patterns motivated the examination of linear combinations of different teleconnection patterns in this study, as it has been previously been used (Seierstad et al., 2007).

Table 5.1 shows the results of the linear combinations. The teleconnection patterns we identify (PNA, NAO, EA and WP) are in accordance with Franzke and 
Feldstein (2005) and Seierstad et al. (2007). Figure 5.5 shows the composite of the SOKc index constructed using the best regression equation for each month, indicated in Table 5.1. The patterns in this figure are in agreement with the ones in Figure 5.2.

\subsubsection{Seeding}

One mechanism in which the changes in the Atlantic that are observed could be explained is through the idea of 'seeding' (Hoskins and Hodges, 2002): at higher levels, storm tracks travel long distances and they may seed other storms, that is, they may provide the background for new storm development and growth in the Atlantic sector. This is the case of the Pacific storms seeding storms in the Atlantic. Figure 5.6 shows that there are more storms over the Atlantic during positive phases of the SOKc - thus indicating more seeding, through the Pacific storms. The seeding could very well change the eddy driven jet, as shown in Figure 5.7.

A definite change in the downstream part of the Atlantic and over the sub-tropical jet region is also noted, with a suggestion that the polar jet is more southerly (see Fig. 5.7). In fact the most coherent signal is in the Atlantic. 


\subsubsection{ECHAM5 Simulations}

In this study we hypothesize that the NAO damping suggested by Yamamoto et al. (2006) is most effective when sea-ice concentration anomalies of consistent sign throughout the winter (from December through February/March) are defined to possess high persistence. The persistence classification matches the sign of the anomaly; that is, positive anomalies Dec-Feb result in positive persistence. This is essentially describing an anomalous season, the impact of which on the atmosphere would exceed that of any one month alone.

Two sets of simulations were conducted to understand more about the influence of the sea-ice in the SOK on the atmospheric circulation and the idea of persistence. The details of the simulations were described in the methodology. Figure 5.9 shows the positive simulation (EPOS) with full sea-ice in the SOK. The negative simulation (ENEG) considered no sea-ice. Figure 5.10 shows the sensible plus the latent heat anomalies in the SOK region.

Figure 5.11 shows the response (full sea-ice minus no sea-ice) in DJF geopotential in $500 \mathrm{hPa}$ and $1000 \mathrm{hPa}$. The shading shows only significant changes at the 95\% significance-level. There is a weak NAO-like response in the Atlantic sectorsignificant in the centers of the response. The response appears to be equivalent barotropic in the Atlantic sector. Reduced sea-ice produces a stronger NAO and viceversa.

Figure 5.12 shows the genesis density, track density, lysis density and mean intensity composites based on the ECHAM5 dataset. It is interesting to note that the 
storm track statistics of February minus December using the NCEP dataset (not shown) has similarities with Figure 5.12. This indicates that the simulation for ECHAM5 positive could be related to the February conditions, more sea-ice. And the ECHAM5 negative simulation could be related to the December conditions, less sea-ice on average.

\subsection{Conclusion}

The relationship between extra-tropical storm tracks in the Northern Hemisphere have been studied based on the changes in sea-ice area over the SOK. There are different mechanisms at play in that region: sea-ice cover over the SOK is influenced by changes in the storm tracks associated with the large-scale circulation (Fang and Wallace, 1994; Honda et al., 1999); this influence can be both ways (Honda et al., 1999); they are also associated with anomalous Aleutian low and Siberian high (Honda et al., 2001); and the presence of sea ice reduces heat and moisture fluxes from the sea surface - cooling the air above (Honda et al., 1999).

Our results show that the sea-ice cover over the Sea of Okhotsk may affect most storm track variables, not only in the North Pacific, but also in the Atlantic. The Atlantic changes confirm the NAO damping suggested by Yamamoto et al. (2006). It is important to note, however, that Yamamoto et al. (2006) pointed out that when they changed only anomalies in the SOK in a modeling simulation, the damping of the NAO signal was not confirmed. This could have been due to their model setup, which was not specified in their paper, but only mentioned. By using the idea of 'persistence', we were able to confirm the NAO damping. 
Changes in SOK could also help explain some of the variability in the GOA region. If local SST is not influencing GOA (Mesquita et al., 2009), then a plausible explanation would be that the large-scale atmospheric changes influence on the sea-ice cover in the SOK and other regions (e.g.: Eurasia) could be at play.

The teleconnection patterns we identify (PNA, NAO, EA and WP) are in accordance with Franzke and Feldstein (2005). And the seeding from the Pacific storms into the Atlantic help change the Atlantic eddy-driven jet, thus altering the transient eddy vorticity fluxes (as shown by Franzke and Feldstein (2005), most low-frequency patterns in the North Atlantic are driven by transient eddy vorticity fluxes) - and it is the lowfrequency vorticity flux and divergence that contribute toward the NAO decay.

The changes in storm tracks related to SOK observed in this study were not only limited to the North Pacific, but it also included important changes in the entire Northern Hemisphere, again linked to the excitation of a stationary Rossby waves over the SOK results echoed in Yamamoto et al. (2006).

With the ECHAM5 simulations we were able to demonstrate the idea of persistence. The SOK damping effects in the NAO are strongest when we have persistence. We also showed that the SOK has a significant effect on the NAO. Sea ice acts as a lid affecting the flux of mass, heat and momentum. It reduces the solar absorption at the surface and it serves as an insulator. Thus changes of sea-ice in the SOK have an impact, since the heat flux is inversely dependent on the thickness. Hence the SOK has an impact in the atmosphere - confirmed by the ECHAM5 experiments. 


\section{Acknowledgements}

We are grateful for NCAR and NSIDC for providing their data sets in the public domain. We would also like to thank the University of Reading and the Bjerknes Centre for Climate Research for the computational resources provided during this study. This work was made possible by the NOAA Grant NA06OAR4600179 "Social Vulnerability to Climate Change in the Alaskan Coastal Zone". Dr. Jürgen Bader's work was supported by the COMPAS Project funded by the Research Council of Norway. The model runs

have been performed at the Norwegian Metacenter for Computational Science (NOTUR). 


\section{References}

Akaike, H. 1974. A new look at the statistical model identification. Automatic Control, IEEE Transactions on 19, 716-723.

Alexander, M. A., Bhatt, U. S., Walsh, J. E., Timlin, M. S., Miller, J. S. and Scott, J. D. 2004. The Atmospheric Response to Realistic Arctic Sea Ice Anomalies in an AGCM during Winter. Journal of Climate 17, 890-905.

Allan, J. C. and Komar, P. D. 2002. Extreme storms on the Pacific Northwest Coast during the 1997-98 El Nino and 1998-99 La Nina. J. Coastal Res. 18, 175-193.

Bengtsson, L., Hodges, K. I. and Roeckner, E. 2006. Storm Tracks and Climate Change. Journal of Climate 19, 3518-3543.

Cavalieri, D., Parkinson, C., Gloersen, P. and Zwally, H. J. 2008. Sea ice concentrations from Nimbus-7 SMMR and DMSP SSM/I passive microwave data. Accessed : 29 June 2009. Boulder, Colorado, USA : National Snow and Ice Data Center. Digital media.

Chang, E. K. M. and Orlanski, I. 1993. On the Dynamics of a Storm Track. Journal of the Atmospheric Sciences 50, 999-1015.

Davidson, R. and MacKinnon, J. G. 2003. Econometric Theory and Methods. Oxford University Press, $768 \mathrm{pp}$.

Deser, C., Walsh, J. E. and Timlin, M. S. 2000. Arctic Sea Ice Variability in the Context of Recent Atmospheric Circulation Trends. J. Climate 13, 617-633.

Deser, C., Magnusdottir, G., Saravanan, R. and Phillips, A. 2004. The Effects of North Atlantic SST and Sea Ice Anomalies on the Winter Circulation in CCM3. Part II : Direct and Indirect Components of the Response. Journal of Climate 17, 877889.

Fang, Z. and Wallace, J. M. 1994. Arctic sea ice variability on a timescale of weeks and its relation to atmospheric forcing. J. Climate 7, 1897-1914. 
Franzke, C. and Feldstein, S. B. 2005. The continuum and dynamics of Northern Hemisphere teleconnections patterns. J. Atmos. Sci. 62, 3250-3267.

Graham, N. E. and Diaz, H. F. 2001. Evidence for Intensification of North Pacific Winter Cyclones since 1948. Bull. Amer. Meteor. Soc. 82, 1869-1893.

Hodges, K. I. 1994. A General Method for Tracking Analysis and Its Application to Meteorological Data. Mon. Wea. Rev. 122, 2573-2586.

Hodges, K. I. 1995. Feature Tracking on the Unit Sphere. Mon. Wea. Rev. 123, 34583465 .

Hodges, K. I. 1996. Spherical Nonparametric Estimators Applied to the UGAMP Model Integration for AMIP. Mon. Wea. Rev. 124, 2914-2932.

Hodges, K. I. 1999. Adaptive Constraints for Feature Tracking. Mon. Wea. Rev. 127, $1362-1373$.

Hodges, K. I., Hoskins, B. J., Boyle, J. and Thorncroft, C. 2003. A Comparison of Recent Re-analysis Data Sets Using Objective Feature Tracking : Storm Tracks and Tropical Easterly Waves. Mon. Wea. Rev. 131, 2012-2037.

Hodges, K. I. 2008. Confidence intervals and significance tests for spherical data derived from feature tracking. Mon. Wea. Rev. 136, 1758-1777.

Honda, M., Nakamura, H., Ukita, J., Kousaka, I. and Takeuchi, K. 2001. Interannual seesaw between the Aleutian and Icelandic lows. Part I : seasonal dependence and life cycle. J. Climate 14, 1029-1042.

Honda, M., Yamazaki, K., Nakamura, H. and Takeuchi, K. 1999. Dynamic and Thermodynamic Characteristics of Atmospheric Response to Anomalous Sea-Ice Extent in the Sea of Okhotsk. J. Climate 12, 3347-3358.

Hoskins, B. J. and Hodges, K. I. 2002. New Perspectives on the Northern Hemisphere Winter Storm Tracks. J. Atmos. Sci. 59, 1041-1061. 
Johnson, C. M. 1980. Wintertime Arctic Sea Ice Extremes and the Simultaneous Atmospheric Circulation. Monthly Weather Review 108, 1782-1791.

Kalnay, E., Kanamitsu, M., Kistler, R., Collins, W., Deaven, D., Gandin, L. et al. 1996. The NCEP-NCAR 40-Year Reanalysis Project. Bull. Amer. Meteor. Soc. 77, 437471.

Key, J. R. and Chan, A. C. K. 1999. Multidecadal Global and Regional Trends in 1000 $\mathrm{mb}$ and $500 \mathrm{mb}$ Cyclone Frequencies. Geophys. Res. Lett. 26, 2053-2056.

Kistler, R., Kalnay, E., Collins, W., Saha, S., White, G., Woollen, J. et al. 2001. The NCEP-NCAR 50-year Reanalysis: monthly means CD-ROM and Documentation. B. Am. Meteorol. Soc. 82, 247-267.

Magnusdottir, G., Deser, C. and Saravanan, R. 2004. The Effects of North Atlantic SST and Sea Ice Anomalies on the Winter Circulation in CCM3. Part I : Main Features and Storm Track Characteristics of the Response. Journal of Climate 17, 857-876.

Mesquita, M. d. S., Atkinson, D. E. and Hodges, K. I. 2009. Characteristics and Variability of Storm tracks in the North Pacific, Bering Sea and Alaska. $J$. Climate In press.

Murray, R. J. and Simmonds, I. 1995. Responses of climate and cyclones to reductions in Arctic winter sea ice. J. Geophys. Res. 100, 4791-4806.

Orlanski, I. and Katzfey, J. 1991. The life cycle of a cyclone wave in the southern hemisphere. Part I : Eddy energy budget. J. Atmos. Sci. 48, 1972-1988.

Orsolini, Y. J. and Kvamstø, N. G. 2009. The role of the Eurasian snow cover upon the wintertime circulation : decadal simulations forced with satellite observations. $J$. Geophys. Res. Accepted.

Overland, J. E. and Pease, C. H. 1982. Cyclone Climatology of the Bering Sea and Its Relation to Sea Ice Extent. Monthly Weather Review 110, 5-13. 
Partington, K., Flynn, T., Lamb, D., Bertoia, C. and Dedrick, K. 2003. Late twentieth century Northern Hemisphere sea-ice record from U.S. National Ice Center ice charts. J. Geophys. Res. 108, 3343, doi : 3310.1029/2002JC001623.

Quadrelli, R. and Wallace, J. M. 2004. A Simplified Linear Framework for Interpreting Patterns of Northern Hemisphere Wintertime Climate Variability. Journal of Climate 17, 3728-3744.

Roeckner, E., Bäuml, G., Bonaventura, L., Brokopf, R., Esch, M., Giorgetta, M. et al. 2003. The atmospheric general circulation model ECHAM 5. In : MPI Report 349 .

Roeckner, E., Brokopf, R., Esch, M., Giorgetta, M., Hagemann, S., Kornblueh, L. et al. 2006. Sensitivity of simulated climate to horizontal and vertical resolution in the ECHAM5 atmosphere model. J. Climate 19, 3771-3791.

Rogers, J. C. and van Loon, H. 1979. The seesaw in winter temperatures between Greenland and Northern Europe. Part II : Some oceanic and atmospheric effects in middle and high latitudes. Mon. Wea. Rev. 107, 509-519.

Seierstad, I. A. and Bader, J. 2008. Impact of a projected future Arctic sea ice reduction on extratropical storminess and the NAO. Clim. Dyn., doi : 10.1007/s00382-008$0431-5$.

Seierstad, I. A., Stephenson, D. B. and Kvamstø, N. G. 2007. How useful are teleconnection patterns for explaining variability in extratropical storminess? Tellus A 59, 170-181.

Simmonds, I. and Budd, W. F. 1991. Sensitivity of the sourthern hemisphere circulation to leads in the Antarctic pack ice. Quart. J. Roy. Meteor. Soc. 117, 1003-1024.

Simmonds, I. and Keay, K. 2002. Surface Fluxes of Momentum and Mechanical Energy Over the North Pacific and North Atlantic Oceans. Meteor. Atmos. Phys. 80, 118. 
Toyota, T., Ukita, J., Ohshima, K. I., Wakatsuchi, M. and Muramoto, K. 1999. A measurement of sea ice albedo over the southwestern Okhotsk Sea. J. Meteorol. Soc. Jpn. 77, 117-133.

Trenberth, K. E. and Hurrell, J. W. 1994. Decadal atmosphere-ocean variations in the Pacific. Clim. Dyn. 9, 303-319.

Ulbrich, U. and Christoph, M. 1999. A shift of the NAO and increasing storm track activity over Europe due to anthropogenic greenhouse gas forcing. Clim. Dyn., 15, 551-559.

Wallace, J. M. and Gutzler, D. S. 1981. Teleconnections in the Geopotential Height Field during the Northern Hemisphere Winter. Monthly Weather Review 109, 784-812.

Weber, T. and Névir, P. 2007. Storm tracks and cyclone development using the theoretical concept of the Dynamic State Index (DSI). Tellus A 60, 1-10.

Yamamoto, K., Tachibana, Y., Honda, M. and Ukita, J. 2006. Intra-seasonal relationship between the Northern Hemisphere sea ice variability and the North Atlantic Oscillation. Geophys. Res. Lett. 33, L14711. 


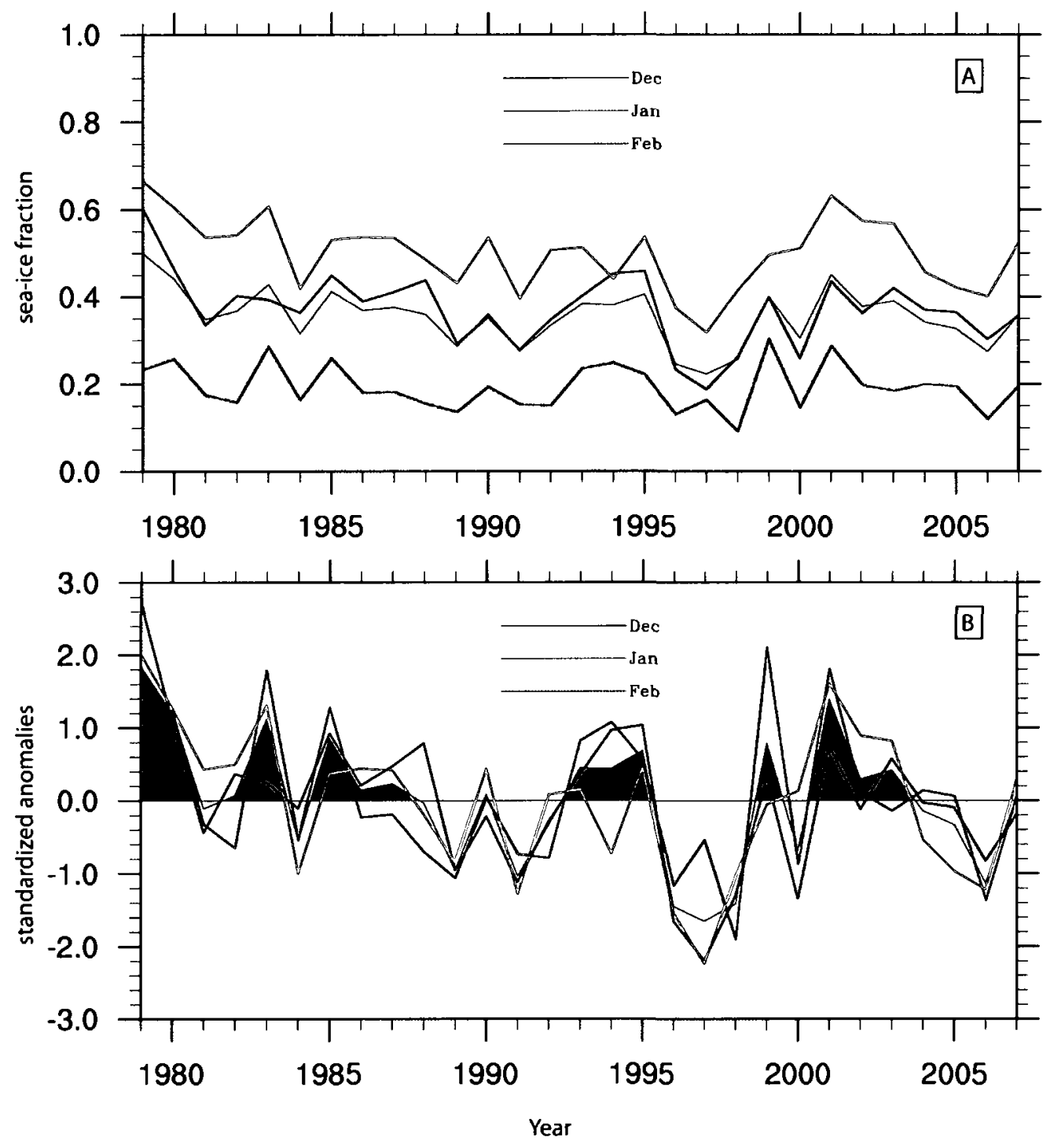

Figure 5.1 - Sea of Okhotsk sea ice concentration

Sea of Okhotsk sea ice concentration: A) sea-ice fraction in the Sea of Okhotsk for December, January and February from 1979 to 2007. 2007. Shading in the background represents the average DJF sea-ice concentration. B) SOKc standardized anomalies for December, January and February. Shading represents the average DJF SOKc. 

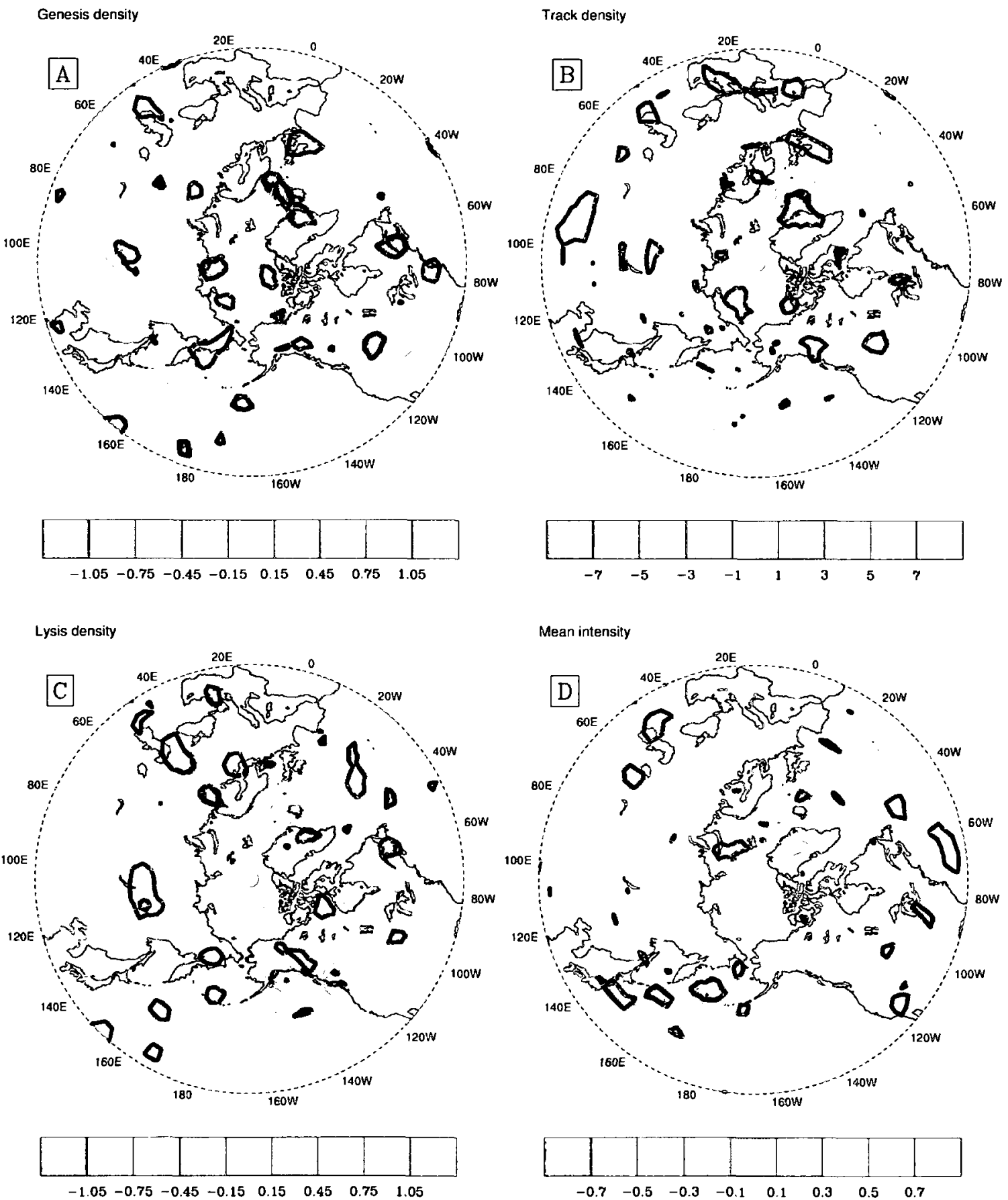

Figure 5.2 - Epoch analysis showing enhanced positive ice anomalies in SOK Difference for SOKc+ minus SOKc- composites of SOKc (1978(9) to 2007). A) genesis density; B) track density; C) lysis density; D) mean track intensity. The thick black lines indicate regions where the $\mathrm{p}$-values are less than $5 \%$. 

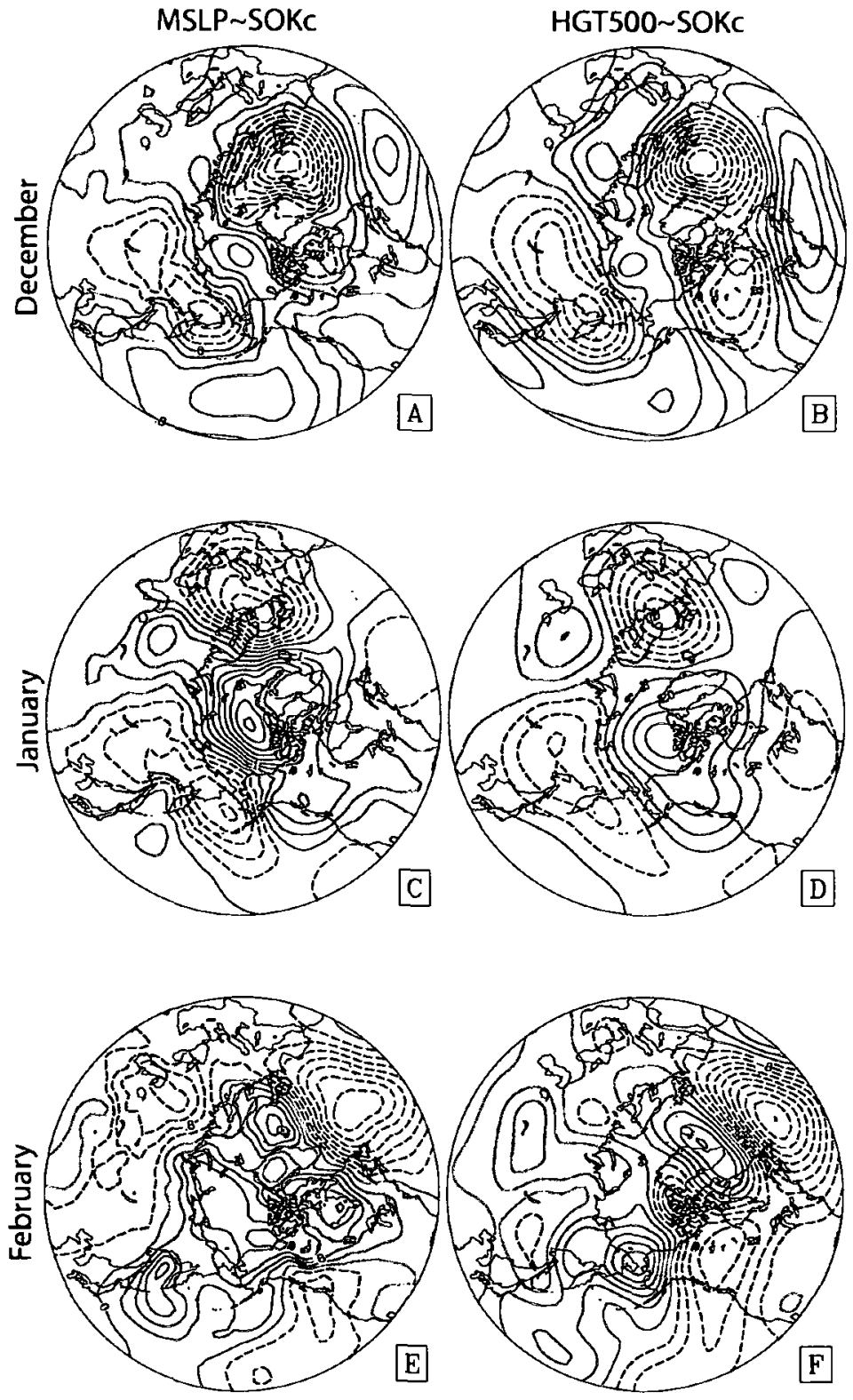

Figure 5.3 - Atmospheric circulation anomalies

SOKc regression plots. Left - monthly MSLP regressed against the monthlystandardized SOKc index for (A) December, (C) January and (E) February, in mb (contour interval: 0.4 ). Right - monthly $500 \mathrm{hPa}$ geopotential height regressed against the monthly-standardized SOKc index for (B) December, (D) January and (F) February, in m (contour interval: 5). Blue, red and magenta lines represent negative, positive and zero values, respectively. 

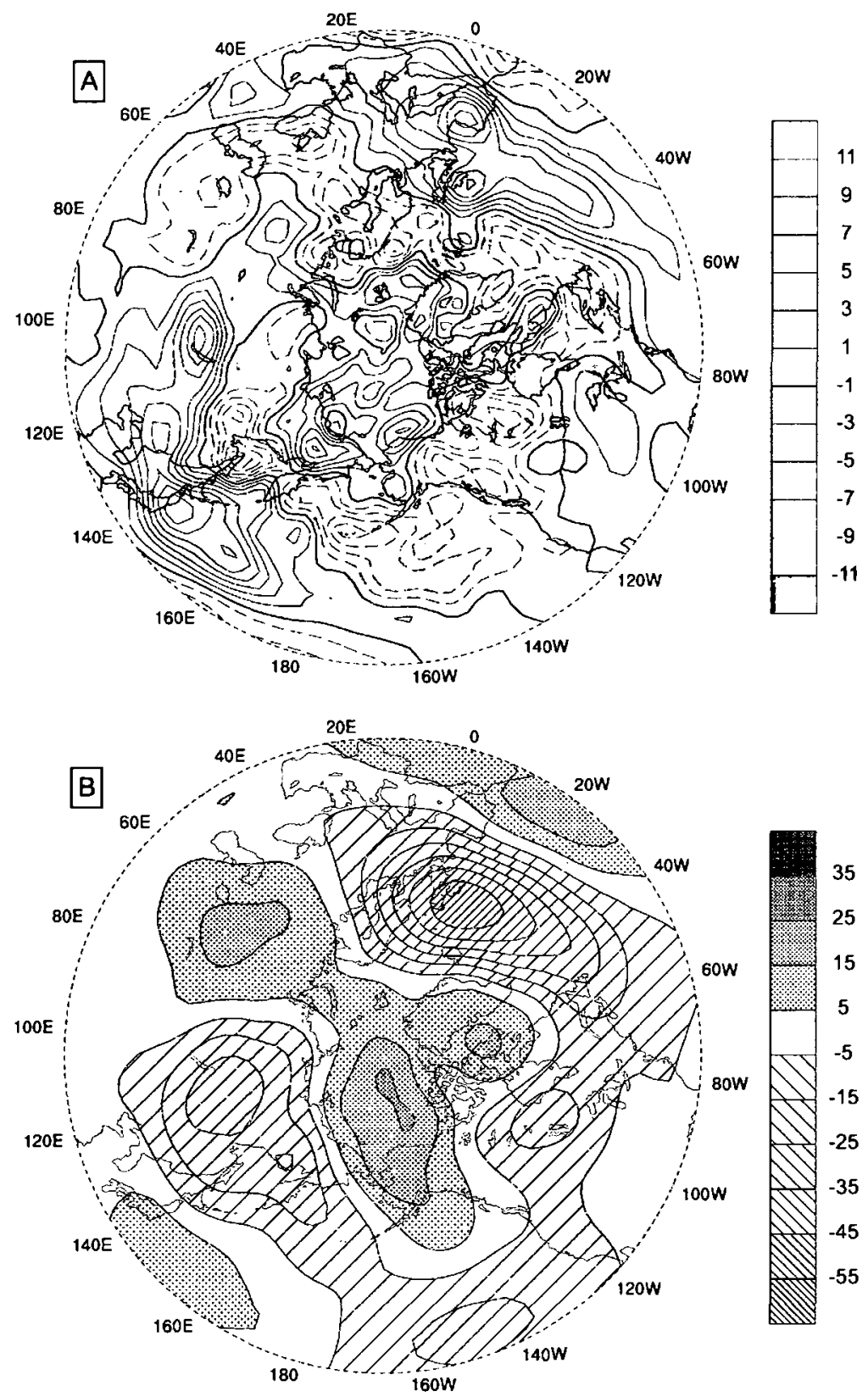

Figure 5.4 - SOKc composites

DJF SOKc weighted composites for 1978(9) to 2007(8): (A) 850-hPa Eady growth rate for the positive (red) minus negative (blue) phases of SOKc. Contour lines represent TDENS for the positive (solid) minus negative (stipled) phases of SOKc. Zero line shown as thick solid line, (B) 500-hPa geopotential height of the positive minus negative phases of SOKc. 

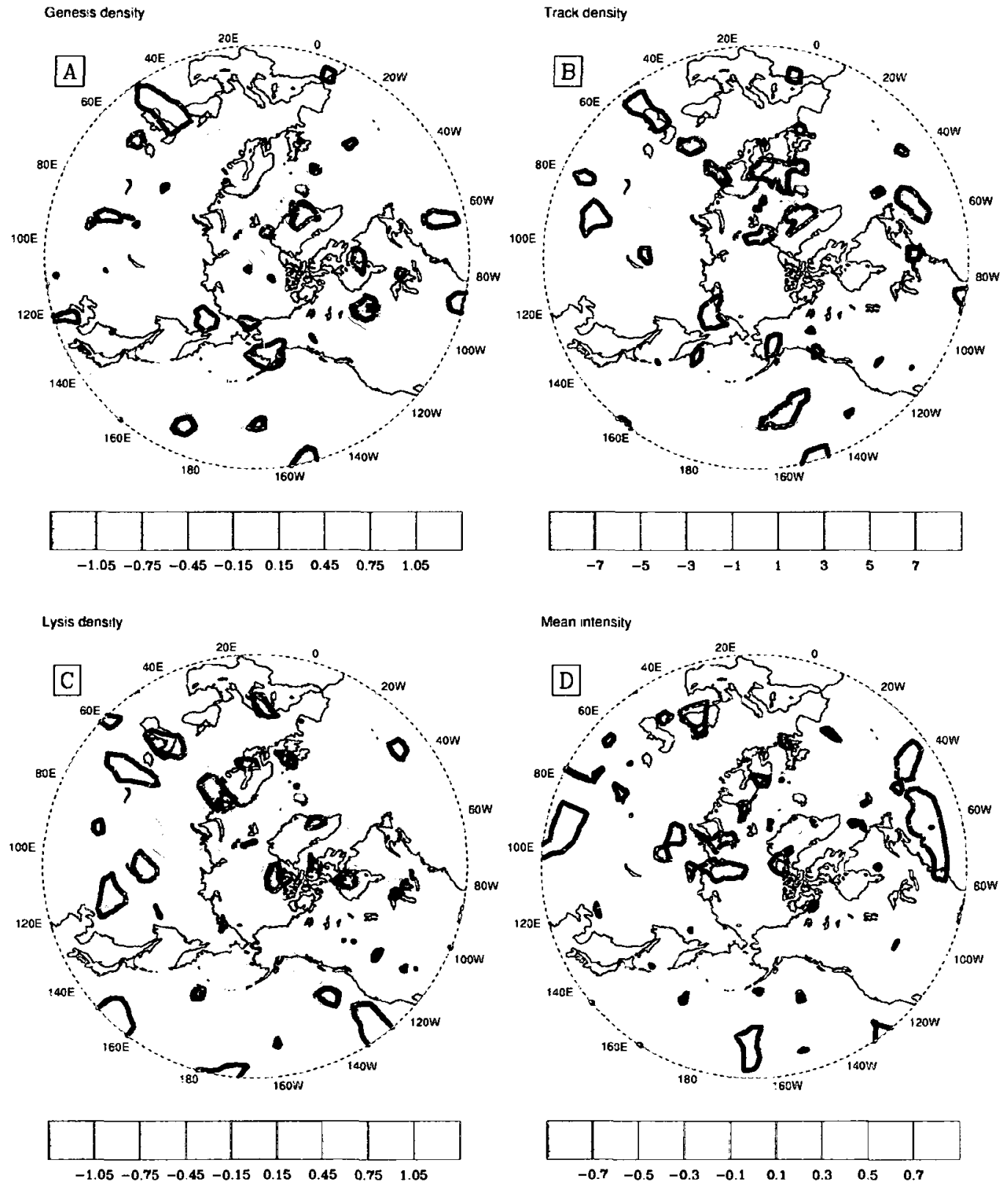

Figure 5.5 - Epoch analysis based on regression equations

Difference for $\mathrm{SOKc}+$ minus SOKc- composites of SOKc constructed from the regression equations (1978(9) to 2007) - See text for details. A) genesis density; B) track density; C) lysis density; D) mean track intensity. The thick black lines indicate regions where the p-values are less than $5 \%$. 

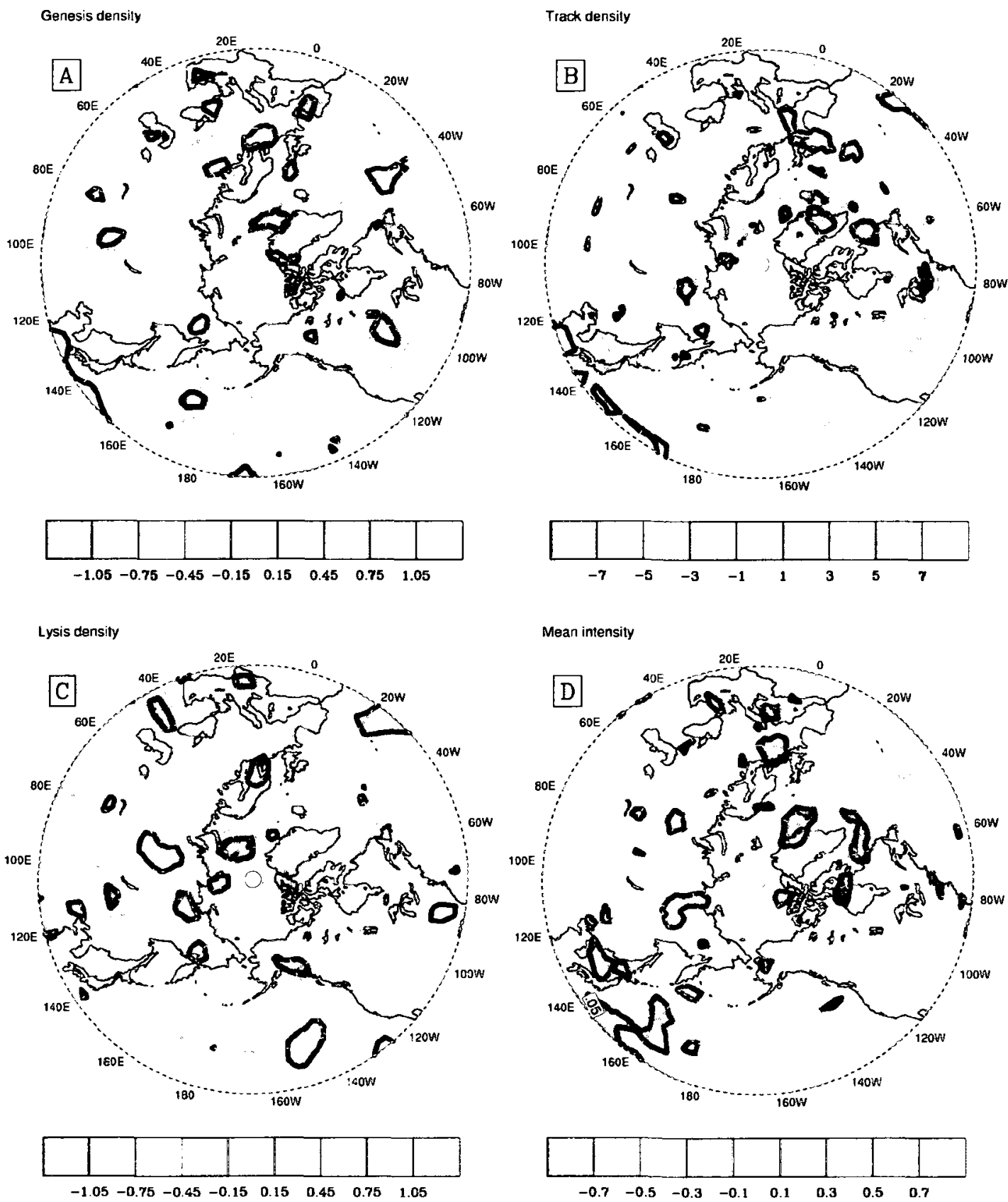

Figure 5.6 - SOKc+ minus SOKc- 250hPa composites

Difference for SOKc+ minus SOKc- composites of SOKc (1978(9) to 2007), regressed at the 250-hPa level. A) genesis density; B) track density; C) lysis density; D) mean track intensity. The thick black lines indicate regions where the p-values are less than $5 \%$. 

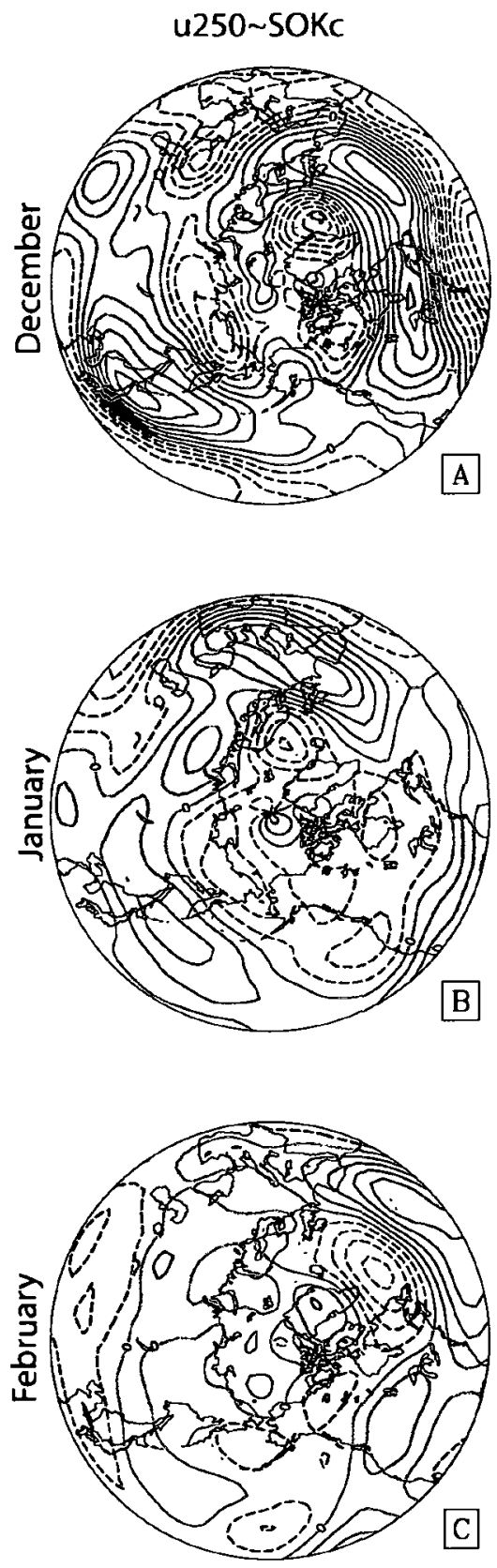

Figure 5.7 - u250 regressed against SOKc

Monthly u250 regressed against the monthly-standardized SOKc index for (A) December, (B) February and (C) March, in $\mathrm{m} / \mathrm{s}$. Red, blue and magenta lines represent positive, negative and zero values, respectively. 

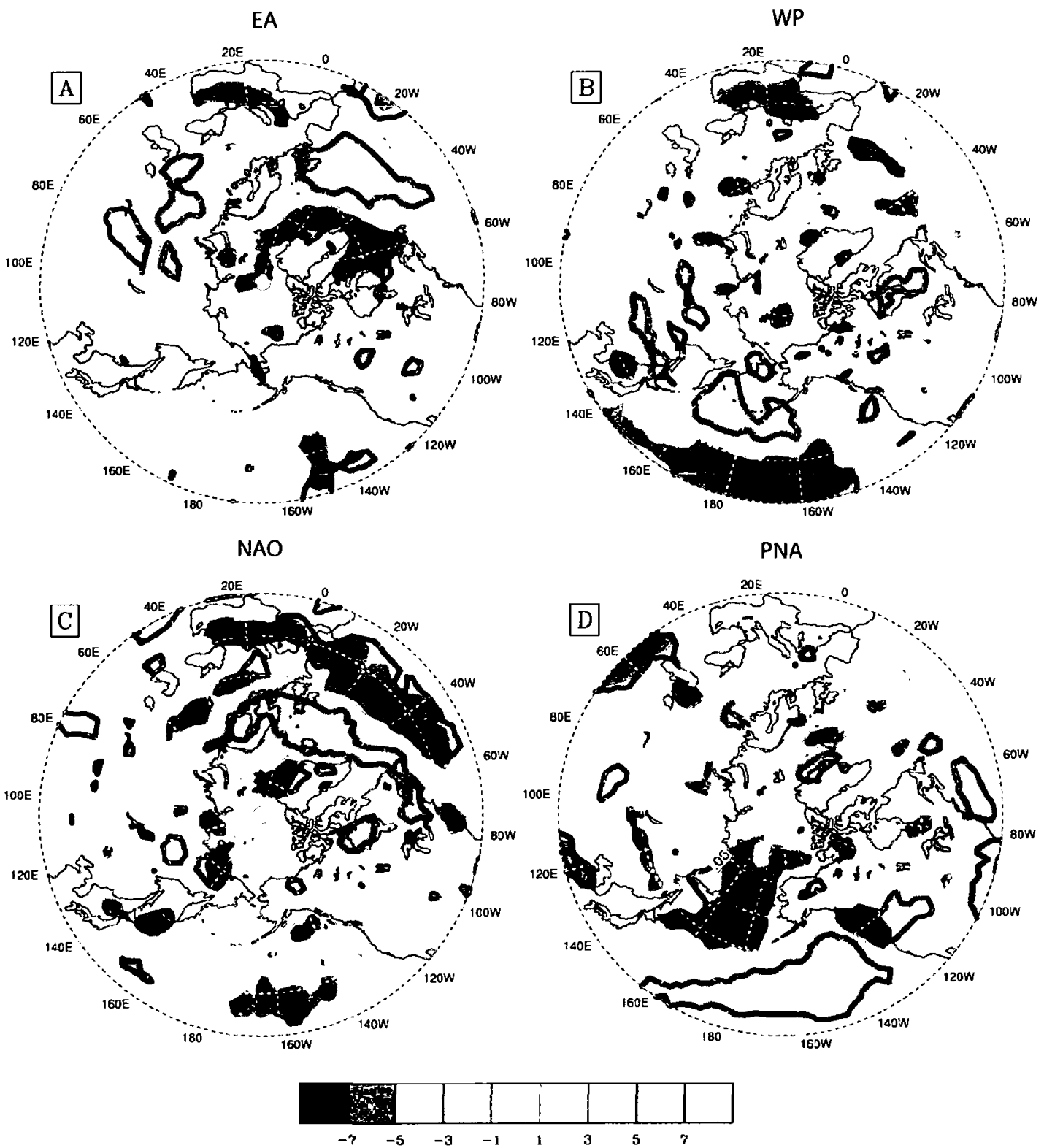

Figure 5.8 - Track density composites

Track density difference composites (positive - negative) for different teleconnections patterns. A) East Atlantic pattern (EA); B) West Pacific pattern (WP); C) North Atlantic oscillation (NAO); d) Pacific/North American pattern (PNA). The thick black lines indicate regions where the p-values are less than $5 \%$. 


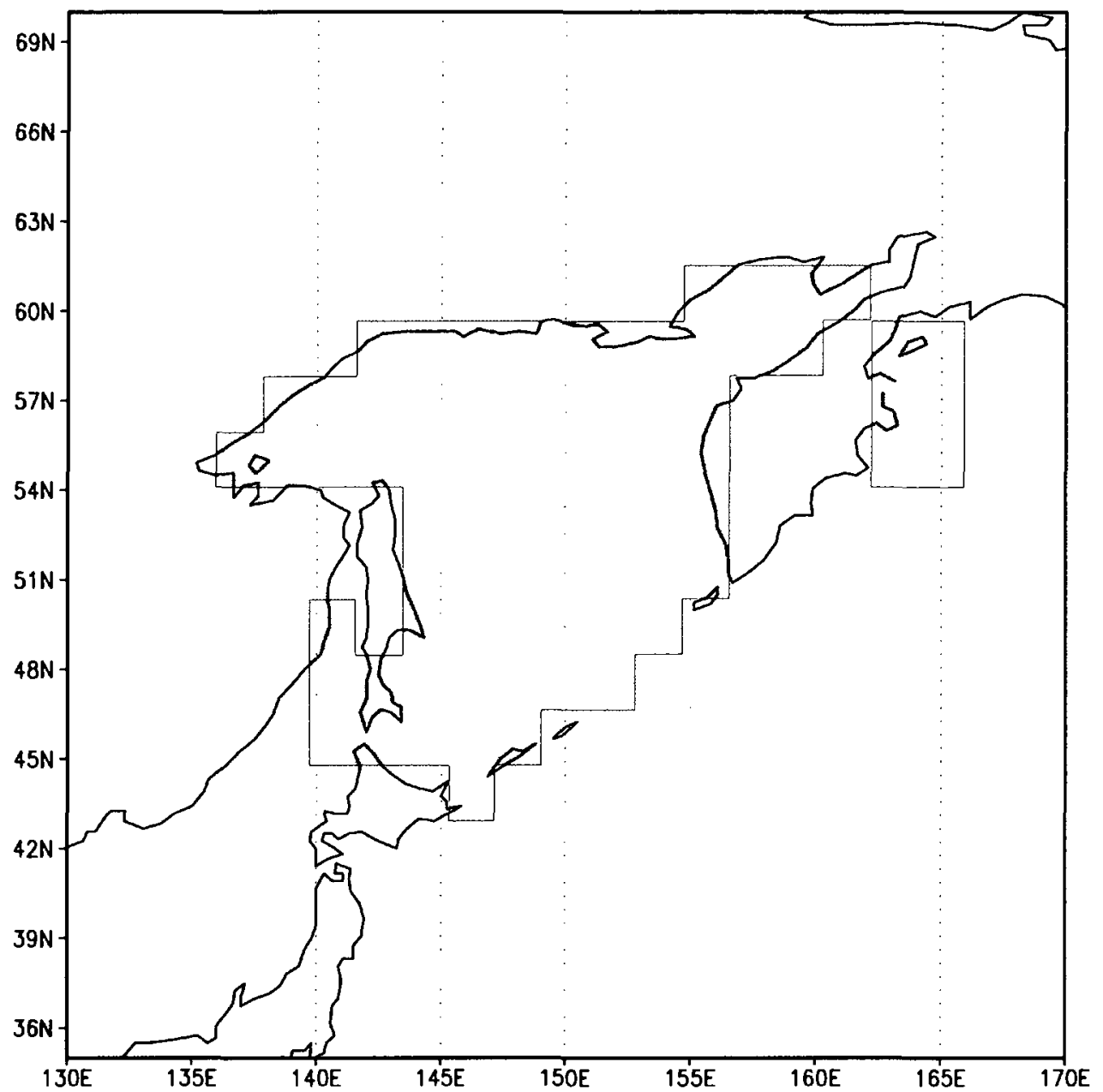

Figure 5.9 - ECHAM5 sea-ice simulation area The sea-ice concentration area used for the ECHAM experiment. Shading represents the sea-ice concentration. The ECHAM negative experiment used a domain with no sea-ice. 


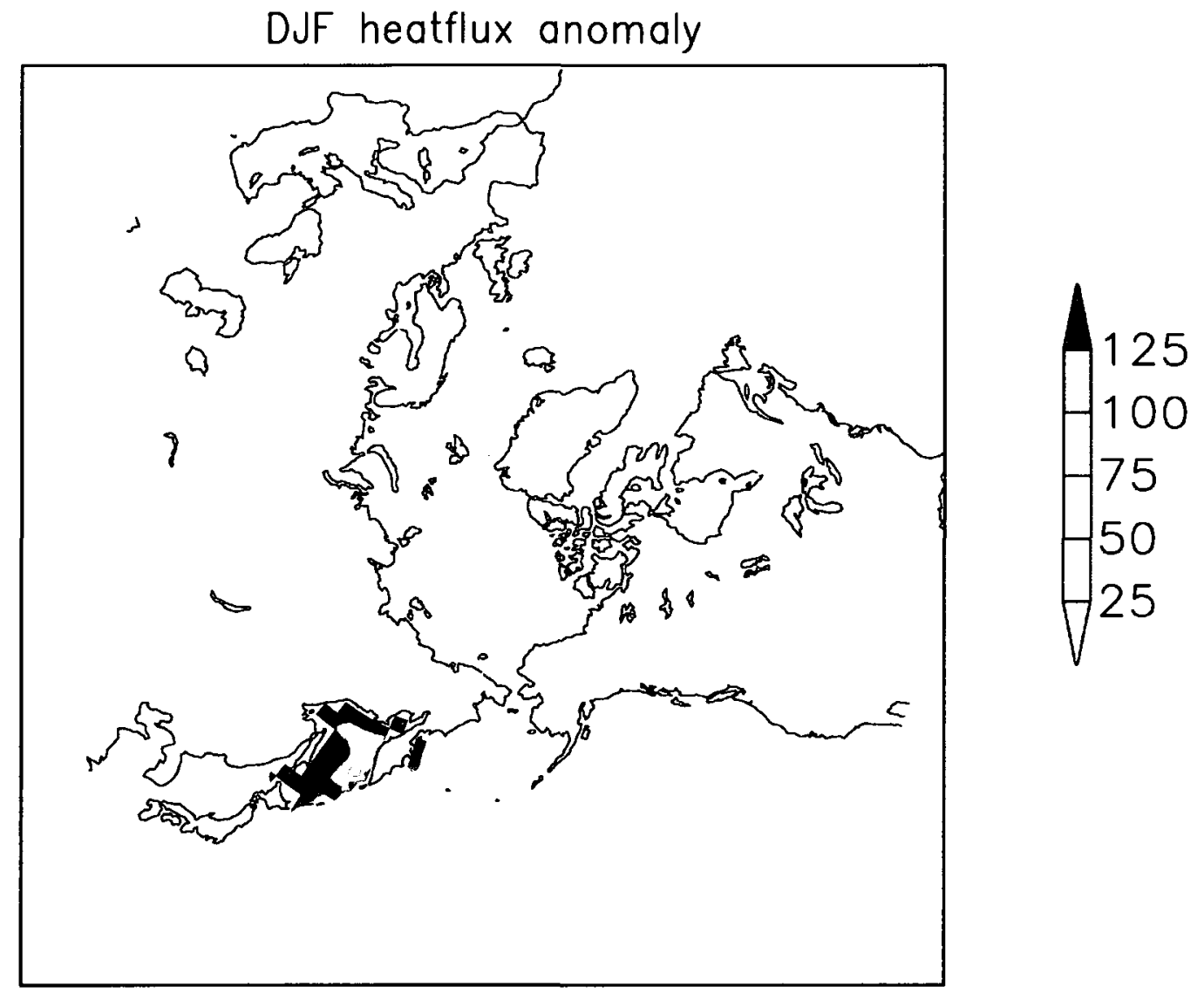

Figure 5.10 - Sensible plus latent heat flux anomalies

The sensible plus the latent heat flux anomalies of the $0 \%$ SIC minus $100 \%$ SIC for the DJF (December, January and February) season. Units in: W/m2. Only positive values are shown. Positive means from the ocean to the atmosphere. 

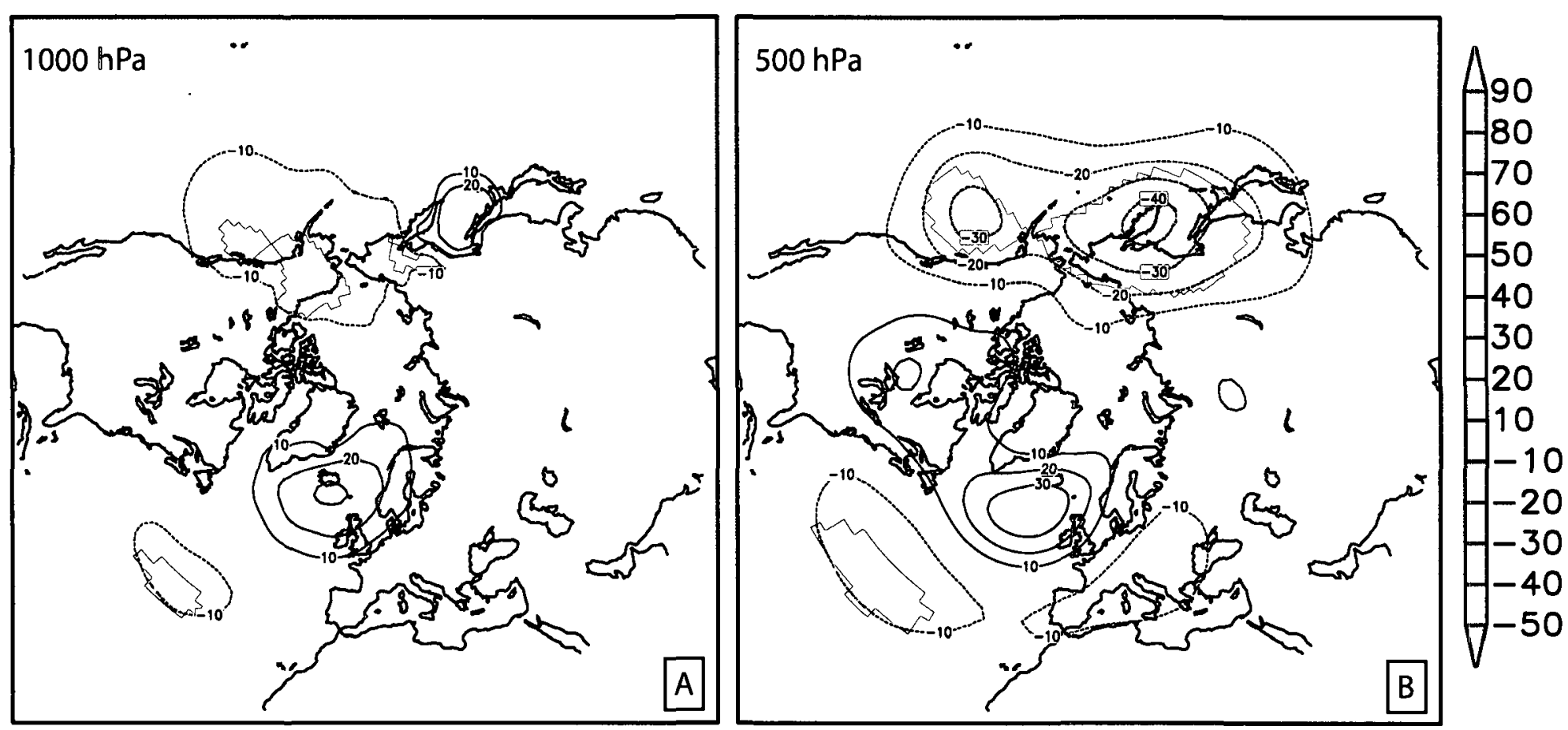

Figure 5.11 - Geopotential height response from ECHAM5

The contours show the simulated mean winter (DJF) geopotential height response in (A) $1000 \mathrm{hPa}$ and (B) $500 \mathrm{hPa}$, between the ECHAM5 runs with a full sea-ice cover in the Sea of Okhotsk and no sea ice in that region. Areas statistically significant at the $5 \%$ level are colored. Unit: gpm. 

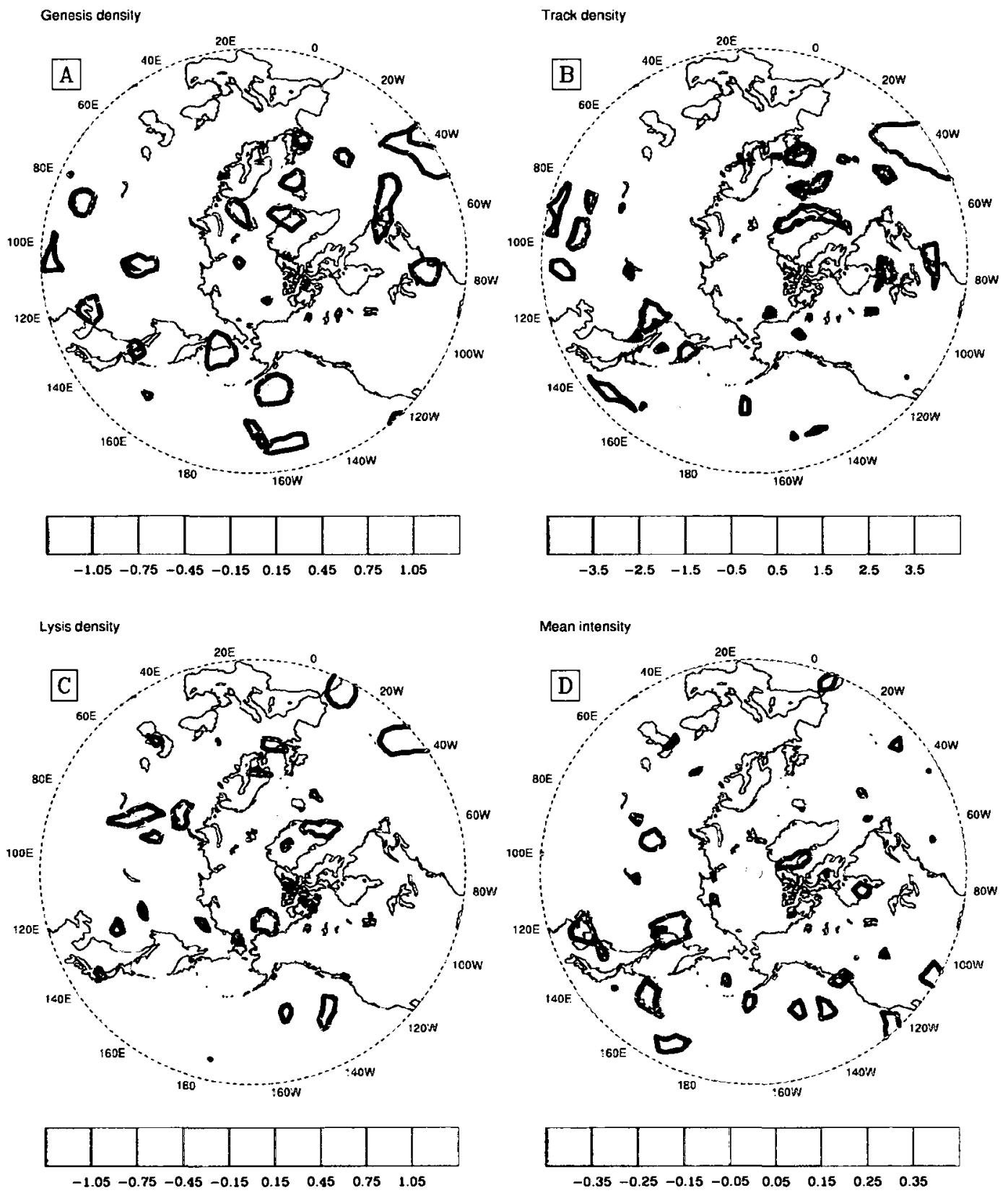

Figure 5.12 - Epoch analysis from ECHAM5 simulations Difference for SOKc+ minus SOKc- composites of SOKc (1978(9) to 2007) tracked using the ECHAM5 relative vorticity dataset. A) genesis density; B) track density; C) lysis density; D) mean track intensity. The thick black lines indicate regions where the pvalues are less than $5 \%$. 
Table 5.1 - SOKc regression equations

SOKc regressed against different teleconnections patterns. Equations are shown for December, January and February and they refer to the period between 1979 to 2008 . The rank represents the three best models among all possible combinations between the teleconnections, trend (year) and no intercept (\#). These models were chosen based on their AIC values.

\begin{tabular}{|c|c|c|c|c|}
\hline & Rank & Equation & p-value & $\mathrm{R}^{2}(\%)$ \\
\hline \multirow{3}{*}{ 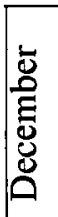 } & 1 & SOKc $\sim \#+9.35 \times 10^{-5} *$ year $-1.87 \times 10^{-2} *$ PNA $+1.88 \times 10^{-2} * \mathbf{N A O}+1.98 \times 10^{-2} * \mathbf{E A}$ & $<0.001$ & 94 \\
\hline & 2 & SOKc $\sim 0.19-1.82 \times 10^{-2} * \mathbf{P N A}+1.87 \times 10^{-2} * \mathbf{N A O}+1.96 \times 10^{-2} * \mathbf{E A}$ & 0.1100 & 12 \\
\hline & 3 & SOKc $\sim 0.19+1.55 \times 10^{-2} * \mathbf{N A O}$ & 0.1065 & 6 \\
\hline \multirow{3}{*}{ 吾 } & 1 & SOKc $\sim 6.80-3.23 \times 10^{-3} *$ year $+3.90 \times 10^{-2} *$ WP $-1.85 \times 10^{-2} *$ NAM & 0.009 & 29 \\
\hline & 2 & SOKc $\sim 6.56-3.11 \times 10^{-3} *$ year $+3.98 \times 10^{-2} * \mathbf{W P}+4.61 \times 10^{-3} *$ POL $-1.92 \times 10^{-2}$ & 0.02 & 26 \\
\hline & 3 & SOKc $\sim 6.71-3.18 \times 10^{-2} *$ year $-9.96 \times 10^{-4} \mathrm{NP}+3.96 \times 10^{-2} * \mathbf{W P}-1.77 \times 10^{-2} * \mathbf{N A M}$ & 0.02 & 26 \\
\hline \multirow{3}{*}{ 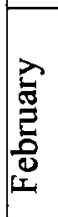 } & 1 & $\mathrm{SOKc} \sim 6.78-3.16 \times 10^{-3} *$ year $-2.25 \times 10^{-2} *$ NAO & 0.097 & 10 \\
\hline & 2 & SOKc $\sim 6.58-3.05 \times 10^{-3} *$ year $-1.04 \times 10^{-2} *$ NAM & 0.078 & 13 \\
\hline & 3 & SOKc $\sim 6.99-3.25 \times 10^{-3} *$ year $-2.51 \times 10^{-2} * \mathbf{N A O}+1.31 \times 10^{-2} * \mathbf{E A}$ & 0.1500 & 9 \\
\hline
\end{tabular}


Chapter 6 New perspectives on the synoptic development of the severe October 1992 Nome storm ${ }^{3}$

\begin{abstract}
Understanding the characteristics of storms that impact the Alaska region is of importance to emergency planning. The 5-7 October 1992 storm was a severe event which cost Nome, a town in Alaska, $\$ 6$ million dollars. We will explore its characteristics with the aid of two established cyclone tracking schemes: the NOAA CPC current operational algorithm and the University of Melbourne algorithm. Manual tracking was performed as a control. The essential features are captured by both algorithms, but they differ in the genesis and lysis location. The NOAA algorithm broke the storm into two separate events. Synoptic development of the storm was influenced by a blocking high that affected how the tracking algorithms handled the event. A synoptic re-examination of this storm is presented in terms of the depth, Laplacian and radius of the system. These new results present a fresh perspective on the intensity and longevity of this dramatic storm.
\end{abstract}

\footnotetext{
${ }^{3}$ Mesquita, M.d.S., D.E. Atkinson, I. Simmonds, K. Keay and J. Gottschalck, 2009: New perspectives on the synoptic development of the severe October 1992 Nome storm. Geophys. Res. Lett., 36, L13808, doi:10.1029/2009GL038824
} 


\subsection{Introduction}

One of the predicted effects of climate change is an increase in storm activity (frequency and intensity [Simmonds et al., 2008]) in the North Pacific, Bering Sea and Arctic Ocean [Graham and Diaz, 2001]. Strong storms are an annual occurrence in the Bering Sea and Gulf of Alaska, with peak activity in the fall/winter season [Fathauer, 1978]. Relatively few of these storms form locally; instead they consist of "old" systems that have moved into the region from the North Pacific and which have undergone a process of re-energization. They represent a serious hazard to users of these marine regions, subsistence and commercial alike, as well as to coastal communities. Nome, a community on coastal western Alaska (Fig. 6.1), is frequently affected: storm-surge accounts from newspapers from 1899 to 1993 indicate severe storms occur most often in October-November, with frequency peaks of catastrophic and property-damaging events occurring in 1900, 1913, 1945-46, 1974 and 1992 [Mason et al. 1996].

For this paper the powerful event of 1992 is examined: it registered average winds of $29 \mathrm{mph}(47 \mathrm{~km} / \mathrm{h})$ and maximum winds of $59 \mathrm{mph}(95 \mathrm{~km} / \mathrm{h})$, with a central pressure minimum of $962 \mathrm{hPa}$ ( $12 \mathrm{Z} 06$ October). The rapidity of its re-energization is indicated by a minimum pressure of $974 \mathrm{hPa}$ only 12 hours earlier. This storm event caused one of the most significant flooding episodes in Nome since that of November 1974 [Fathauer, 1975], and represents the highest storm surge since the Nome water level gauge data became available in 1992 [Blier et al., 1997]. This surge placed the peak water level only $1.8 \mathrm{~m}$ below the top of the town seawall, which is about $8.3 \mathrm{~m}$ high $(7.9 \mathrm{~m}$ in the business district). 
Storms research has focused on tracking individual events to identify typical trajectories and intensities in what is termed a "quasi-Lagrangian" approach [Simmonds et al., 2008]. They identify local minima/maxima of storm diagnostic parameters, such as sea level pressure, within blocks of grid points [Murray and Simmonds, 1991; Serreze et al., 1993; Sinclair, 1994; Zhang et al., 2004]. Arguably the most commonly applied algorithm for the Alaska region is that operated at NOAA/CPC [Gottschalck, 2008], which uses the method of Serreze et al. [1993] and Serreze [1995] ("GS" hereafter).

An alternative approach was developed at The University of Melbourne [Murray and Simmonds, 1991; Simmonds et al., 1999; Simmonds and Keay, 2000], hereafter the "Melbourne" algorithm. This approach, like GS, analyses MSLP. However, open lows are incorporated into the storm lifecycle which prevent possibly inappropriate timeseries breaks if temporary weakening to an open-low state occurs. It has been applied to studies in both hemispheres [Murray and Simmonds, 1991; Simmonds and Keay, 2000; Pinto et al., 2005; Simmonds et al., 2008] but not specifically for Alaska.

The primary goal of the present paper is to study the evolution of this dangerous storm from the perspective of two robust storm-tracking algorithms. Selection of a stormtracking algorithm that can handle a broad range of storm life cycle phases is an important first step to better serve Alaskans in this time of potentially increasing storm activity [Mesquita et al., 2008]. 


\subsection{Methodology and Data Sets}

The two tracking algorithms, GS and Melbourne, are contrasted using a specific case study, the event of September-October 1992. Both algorithms were run using 6hourly mean sea level pressure data from the NCEP/NCAR reanalysis (R1) [Kistler et al., 2001] for 1992 .

The GS algorithm tracks storms by finding the center of a "closed low" pressure system, i.e., a grid point is surrounded by points with higher values and appears as a closed line on a mean sea level pressure map. A threshold of $1 \mathrm{mb}$ was selected as the minimum difference between the central point and the surrounding ones. A choice of threshold below $2 \mathrm{mb}$ is ideal [Eichler and Higgins, 2006]. There are two possible weaknesses with the 'GS' method: mean storm count can be overestimated because it does not account for the entire storm life-cycle [Pinto et al., 2005], and that it only considers the average value of MSLP between 9 grid points with thresholds [Gulev et al., 2001]. GS does not track the system when the storm center is no longer closed. This can be problematic because "open-low" systems, i.e., a low pressure at a weakening stage when a grid point is not completely surrounded by higher value points, may continue to cause damage and they may also reenergize into closed systems [Bergeron, 1950]. In the latter case, GS could return erroneously high storm counts. Thus an algorithm that accounts for open low systems should return storm climatology results that better approximate reality.

The Melbourne tracking algorithm provides information about system type open/closed - and relative strength - weak/strong. An open/closed system is defined by 
comparing the Laplacian of pressure at each grid point with its neighbors [Murray and Simmonds, 1991; Simmonds et al., 2008]. When a possible low is found, the location of the pressure minimum is interpolated by iterative approximation to the center of an ellipsoid of best fit to the pressure surface. An open system is searched for when a closed center cannot be determined by the ellipsoid. A system is classified as "weak/strong" based on the "concavity criterion" [Simmonds et al., 2008]: when the average value of the Laplacian exceeds $0.2 \mathrm{hPa}\left({ }^{\circ} \text { lat }\right)^{-2}$ over a radius of $2^{\circ}$ lat, the storm is included in the count. If the Laplacian value is between 0.2 and $0.7 \mathrm{hPa}\left({ }^{\circ} \text { lat }\right)^{-2}$, the storm is considered "weak", and higher than $0.7 \mathrm{hPa}\left({ }^{\circ} \text { lat }\right)^{-2}$, "strong". The depth (in $\mathrm{hPa}$ ) and the Laplacian of the pressure [in $\mathrm{hPa}\left({ }^{\circ} \text { lat }\right)^{-2}$ where $1^{\circ}$ lat (degree latitude) is approximately $111 \mathrm{~km}$ ] will be shown for the Melbourne algorithm in order to describe the lifetime of this storm. Finally, manual tracking was also performed to act as a control for this event. 


\subsection{Results}

\subsubsection{The Storm of 5-7 October 1992}

The low formed initially over eastern China while a large blocking high was sitting over the North Pacific (01 October 1992 at 12UTC, not shown). Between 01 October and 04 October the storm strengthened and moved towards the Sea of Okhotsk and the blocking high became larger, developing a ridge extending from the North Pacific up through the state of Alaska (Figure 6.1a,b: 04 October 1992 at 18UTC). The surface low was accompanied at the $500 \mathrm{hPa}$ level by a ridge extending north into the Bering Sea (Figure 6.1b). Between 04 October and 06 October the low came under the influence of the high and was diverted northward, over far eastern Russia (Fig 6.2). By 06 October the blocking system has weakened and the 500hPa ridge displaced eastward, allowing the storm to strengthen and move into the north Bering Sea (Figure 6.1c,d: 06 October 1992 at 06UTC). Eighteen hours later the storm has moved into the south Chukchi Sea (Figure 6.1: 07 October 1992 at 00UTC) with a gradient presentation favourable for development of large surge at Nome.

This event was tracked using three different methods (Fig. 6.2). The GS track starts twelve hours later and finishes (one) two days earlier than the (manual) Melbourne track. All three tracks exhibited similar features and positions throughout their lifecycles. The retrograde shift of 02-03 October was well represented by manual and Melbourne, less so by GS.

By 09 October at $18 \mathrm{Z}$ the storm was still a strong closed system, transitioning to a weak closed system by 10 October at $00 \mathrm{Z}$. From there on until 11 October at $00 \mathrm{Z}$, it 
became a weak open system until lysis. The last time steps of the Melbourne track show the most discrepancy when compared to manual, largely because use of the Laplacian of the pressure allows the algorithm to track more accurately the position of the storm center as compared to the manual tracking of the system, which was based on visual assessment of contours.

The most apparent difference amongst the tracks is the break identified by GS on 05 October (Fig. 6.2). The tracking break, situated over the Chukotka region in Russia at $18 \mathrm{Z} 05$ October, was likely triggered in the GS algorithm by a temporary weakening of the system to a strong open system caused by interaction with orography as described in section 6.4 .

Figure 6.3 shows the life history of the storm track. The pressure time series (dashed line) shows that the storm started with a central pressure of $1003 \mathrm{hPa}$ and then it had a slight increase of $6 \mathrm{hPa}$ in 42 hours. After that, the central pressure dropped to its minimum value of $968 \mathrm{hPa}$ on 06 October at $18 \mathrm{Z}$. This represented a drop of $41 \mathrm{hPa}$ from the maximum value within 102 hours. During the period of weakening it increased again to $998 \mathrm{hPa}$ by 10 October at $06 \mathrm{Z}$. After a brief drop to $996 \mathrm{hPa}$ the system dissipated.

The depth and the Laplacian parameters (Figure 6.3) represent a measure of storm intensity [Simmonds et al., 2008]. From the beginning of the storm until 05 October at $06 \mathrm{Z}$, the intensity oscillated without showing an overall trend. It is from that point on that both the depth and the Laplacian rapidly increased over 24 hours. The maximum depth value $(23.21 \mathrm{hPa})$ occurred on 06 October at $18 \mathrm{Z} ; 36$ hours earlier the minimum value 
was $4 \mathrm{hPa}$. The maximum Laplacian value occurred on 07 October at $06 \mathrm{Z}(2.77 \mathrm{hPa}$ $\left.\left({ }^{(} l a t\right)^{-2}\right)$. Both the depth and the Laplacian dropped in value until close to the end of the storm lifetime.

The propagation speed (Figure 6.3) shows a similar pattern to the intensity parameters. As the storm moved from sea to land between 05 October and 06 October, the speed decreased and increased afterwards, covariant with the Laplacian and depth parameters.

\subsubsection{The Role of the Blocking High}

The main reason the storm weakened to an open system was due to the influence of a high-pressure system located along Alaska, the Aleutian Islands and part of the North Pacific Ocean in a SW-NE axis (Figure 6.1a). Continued progression of the storm northeastward along its maritime track was interrupted as it was diverted by the blocking high system until 05 October at $12 \mathrm{Z}$. The high and its ridge, however, then set the stage for the initial phase of the rapid storm strengthening. As the low moved up against the high a strong gradient was established along the west side of the high/ridge in which a strong southerly flow developed (see arrow, figure 6.1a) that brought destabilizing warmth and water vapour to the storm at lower levels. 


\subsection{Discussion and Conclusion}

The fact that the blocking high system helped shape the Nome storm of 1992 is in agreement with Blier et al. [1997]. This seems to be the case for storms that cause

flooding in Nome and other areas of Alaska [Fathauer, 1975, 1978; Blier et al., 1997]. Other authors confirm that the North Pacific storms 'frequently' move into the Chukchi Sea as blocking ridges develop over the Aleutian Islands [Wilson and Overland, 1987; Salmon, 1992; Mason et al., 1996]. This cyclone-anticyclone configuration is similar to the 'classic' type of events associated with cold outbreaks south of Australia [Simmonds and Rashid, 2001]. Thus in the North Pacific context it would appear that a blocking high with ridge serves to a) interact with an approaching low to develop a relatively steep pressure gradient along the ridge west side, b) entrain warmth and moisture in the ensuing strong southerly flow and advect it into the low, and to c) deflect these now revitalized storms to the northeast, towards the Aleutian Islands and Bering Sea. Sea surface temperature plots for 1992 (not shown) suggested anomalous SST warmth in the September-October period in the mid-North Pacific. On 05 October at $12 Z$, the storm weakened and became an open system. The weakening was caused by its trajectory over land and interaction with orography.

The strengthening of the storm during 05-06 October displaced the surface ridge to the east. In addition to the mechanisms described above it is possible that storm reenergization was also facilitated by a drawing of kinetic energy from the background flow associated with the ridge break up process. Storm re-energization may have been further aided by increased local availability of water vapour as it traversed several water 
bodies: the Sea of Okhotsk and the Gulf of Anadyrsk. As the system progressed towards the Bering Sea the southerly moisture and heat flow dissipated with the disruption of the ridge but favourable conditions developed aloft, specifically, a short-wave trough with vorticity and divergence maxima to the east, positioned over the center of the surface low.

GS and Melbourne captured the main trajectory features of the 1992 Nome storm. They differ in the genesis and lysis location, which is not unusual when using different algorithms [Raible et al., 2008]. The fact that GS split the storm into two is not necessarily in error and may point to an alternate explanation of event evolution; that is, that there were in fact two separate systems. As the storm moved from the Sea of Okhotsk, it died as it reached the cold land temperatures over Russia. A separate system started over the Bering Sea moving towards the Chukchi Sea - a region of high baroclinicity due to the gradient of temperature in that region, akin to processes along the US eastern seaboard.

Blier et al. [1997] show the event as one system and not two (their Figure 4). In the present case study it is felt that the Melbourne algorithm provides additional insights into the storm evolution, in terms of its genesis location and longevity, via the description of open and closed centers, as well as variables like Laplacian and depth.

The concept of what a "storm" really is represents an important issue when considering automated classification methods. The way a tracking algorithm defines a "storm" clearly sets the context for the tracking results. In this paper, we do not advocate one system as being better than the other but instead echo Leonard et al. [1999, p. 180]: 
"In carrying out an intercomparison of depression tracking schemes great care must be taken not to draw misleading conclusions about the merits and value of a particular scheme, since different users of the software will have different requirements". Hence, a scheme should be chosen according to the user's needs and type of research. 


\section{Acknowledgements}

We are grateful for NCAR for providing their data sets in the public domain. This work was made possible by grants from the Center for Global Change \& Arctic System Research and NOAA Grant NA06OAR4600179 "Social Vulnerability to Climate Change in the Alaskan Coastal Zone". We appreciate comments made on a first draft by two anonymous reviewers, which strengthened the paper. We would also like to thank Mr. Ted Fathauer, NOAA's National Weather Service, for useful discussions. 


\section{References}

Bergeron, T. (1950), De Tropiska Orkanernas Problem, Kungl. Boktr. P. A. Norstedt \& Soener, Stockolm.

Blier, W., et al. (1997), Storm surges in the region of western Alaska, Mon. Wea. Rev., 125, 3094-3108.

Eichler, T., and W. Higgins (2006), Climatology and ENSO-related variability of North American extratropical cyclone activity, J. Clim., 19, 2076-2093.

Fathauer, T. F. (1975), The great Bering Sea storms of 9-12 November 1974, Weatherwise, 28, 76-83.

Fathauer, T. F. (1978), A forecast procedure for coastal floods in Alaska, National Oceanic and Atmospheric Administration, U.S. Department of Commerce, NOAA Tech. Memo NWS AR-23, 27.

Gottschalck, J. (2008), NOAA storm track webpage: http://www.cpc.ncep.noaa.gov/products/precip/CWlink/stormtracks/mstrack.shtml.

Graham, N. E., and H. F. Diaz (2001), Evidence for Intensification of North Pacific Winter Cyclones since 1948, Bull. Amer. Meteor. Soc., 82, 1869-1893.

Gulev, S. K., et al. (2001), Extratropical cyclone variability in the Northern Hemisphere winter from the NCEP/NCAR reanalysis data, Climate Dynamics, 17, 795-809.

Kistler, R., et al. (2001), The NCEP-NCAR 50-year Reanalysis: monthly means CDROM and Documentation, B. Am. Meteorol. Soc., 82, 247-267.

Leonard, S. R., et al. (1999), An assessment of three automatic depression tracking schemes, Meteorol. Appl., 6, 173-183.

Mason, O. K., et al. (1996), The periodicity of storm surges in the Bering Sea from 1898 to 1993, based on newspaper accounts, Climatic Change, 34, 109-123. 
Mesquita, M. d. S., et al. (2008), Climatological properties of summertime extra-tropical storm tracks in the Northern Hemisphere, Tellus A, 60, 557-569.

Murray, R., and I. Simmonds (1991), A Numerical Scheme for Tracking Cyclone Centres from Digital Data. Part I: Development and Operation of the Scheme, Aust. Met. Mag., 39, 155-166.

Pinto, J. G., et al. (2005), Sensitivities of a cyclone detection and tracking algorithm: individual tracks and climatology, Meteor. Z., 14, 823-838(816).

Raible, C. C., et al. (2008), Northern Hemisphere extratropical cyclones: a comparison of detection and tracking methods and different reanalyses, Mon. Wea. Rev., 136, 880-897.

Salmon, D. K. (1992), On interannual variability and climatic change in the North Pacific, Unpublished Ph.D. dissertation, Institute of Marine Science, University of Alaska, Fairbanks.

Serreze, M. C., et al. (1993), Characteristics of Arctic Synoptic Activity, Met. Atmos. Phys., 51, 147-164.

Serreze, M. C. (1995), Climatological Aspects of Cyclone Development and Decay in the Arctic, Atmos-Ocean, 33, 1-23.

Simmonds, I., et al. (1999), A refinement of cyclone tracking methods with data from FROST, Aust. Met. Mag., Spec. Ed., 35-49.

Simmonds, I., and K. Keay (2000), Mean southern hemisphere extratropical cyclone behaviour in the 40-year NCEP-NCAR reanalysis, J. Clim., 13, 873-885.

Simmonds, I., and H. A. Rashid (2001), An investigation of a dramatic cold outbreak over southeast Australia, Aust. Met. Mag., 50, 249-261.

Simmonds, I., et al. (2008), Arctic climate change as manifest in cyclone behavior, $J$. Clim., 21, 5777-5796. 
Sinclair, M. R. (1994), An objective cyclone climatology for the Southern Hemisphere, Mon. Wea. Rev., 122, 2239-2256.

Wilson, J. G., and J. E. Overland (1987), Meteorology, in The Gulf of Alaska: Physical Environment and Biological Resources, edited by D. W. Hood and S. T. Zimmerman, pp. 31-56, National Oceanic and Atmospheric Administration, Minerals Management Study OCS 86-0095.

Zhang, X., et al. (2004), Climatology and Interannual Variability of Arctic Cyclone Activity: 1948-2002, J. Climate, 17, 2300-2317. 


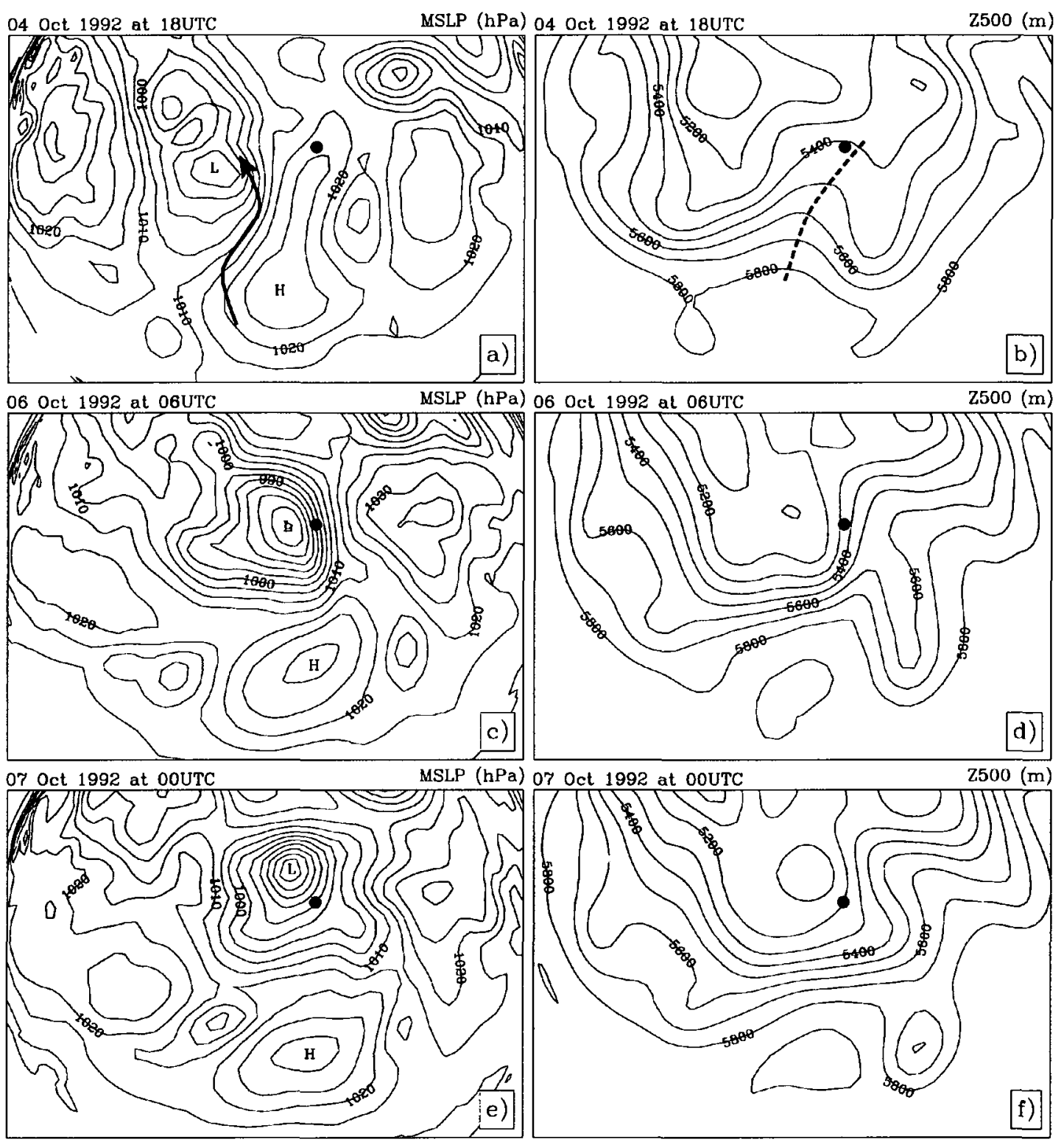

Figure 6.1 - Nome storm MSLP and 500hPa geopotential height plots

NCEP/NCAR mean sea level pressure and $500 \mathrm{hPa}$ geopotential height at three times in the life of the Nome storm: 04 October 1992 at 18UTC (a, b); 06 October 1992 at 06UTC (c, d) and 07 October 1992 at 00UTC (e, f). Contour interval: 5hPa for MSLP and $100 \mathrm{~m}$ for $\mathrm{Z} 500$. Only the case-study storm and the stationary high are labelled in the plot: ' $L$ ' and ' $H$ ' respectively. Heavy arrow shows moisture flow trajectory. Heavy dashed line shows 500 ridge axis. Nome is indicated with the dot. 


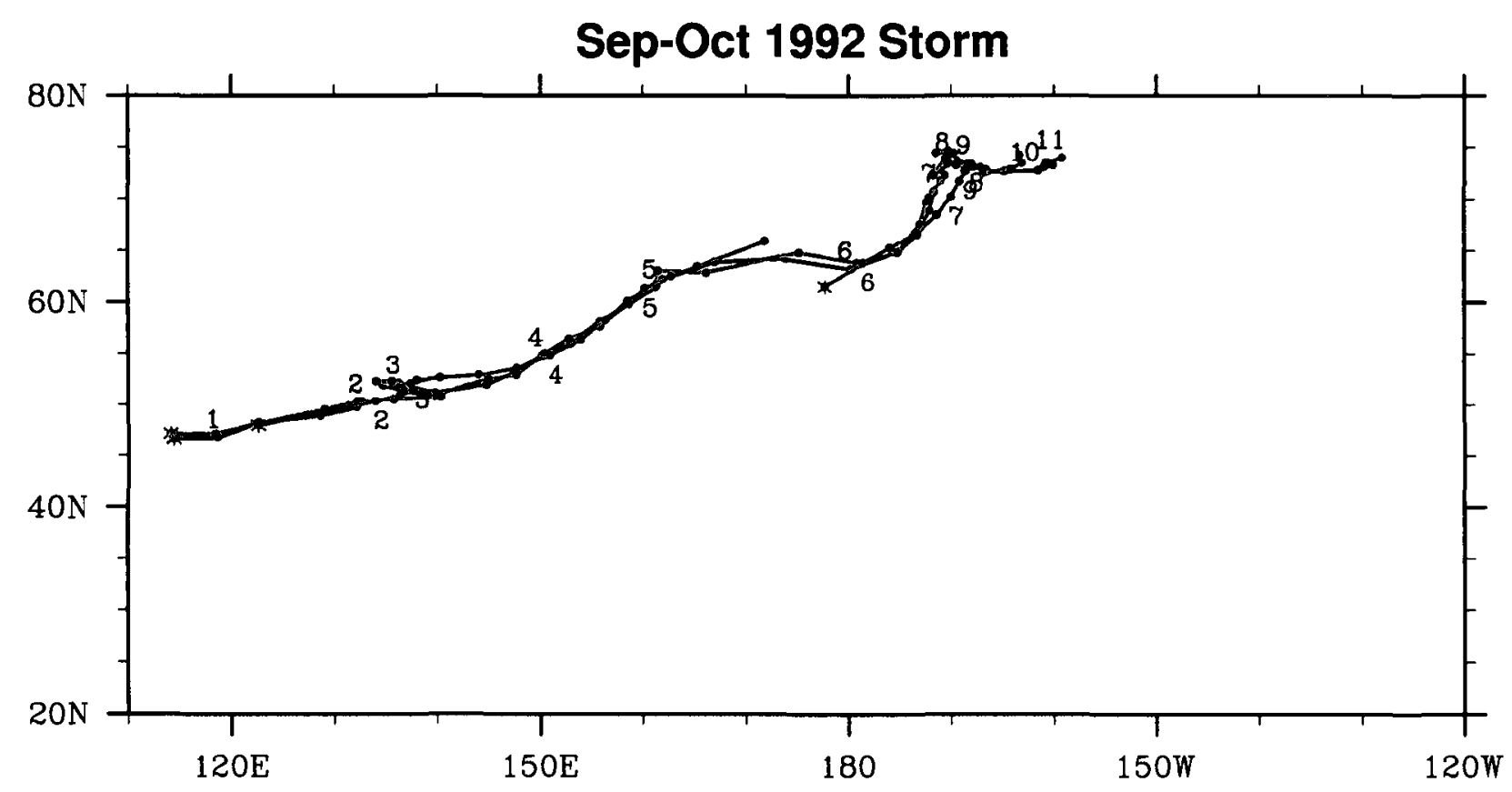

Figure 6.2 - Nome storm from three tracking methods

The September-October 1992 storm represented by three tracking techniques. The manually tracked system (red line) persists from 30 September 1992 at $18 \mathrm{Z}$ through 10 October 1992 18Z. The Melbourne algorithm (blue line) shows the storm persisting from 30 September 1992 at $18 Z$ through 11 October 1992 at 00Z. The GS algorithm (green line) shows two separate systems: the first starting $06 Z 01$ Oct. 1992 through $18 \mathrm{Z} 05$ Oct. 1992, and the second starting at $18 \mathrm{Z} 05$ Oct 1992 through $12 \mathrm{Z} 09$ Oct. 1992. The stars indicate the start point of the storms, the dots represent $6 \mathrm{~h}$-interval time steps and the numbers represent the days in October 1992 at 00Z. 


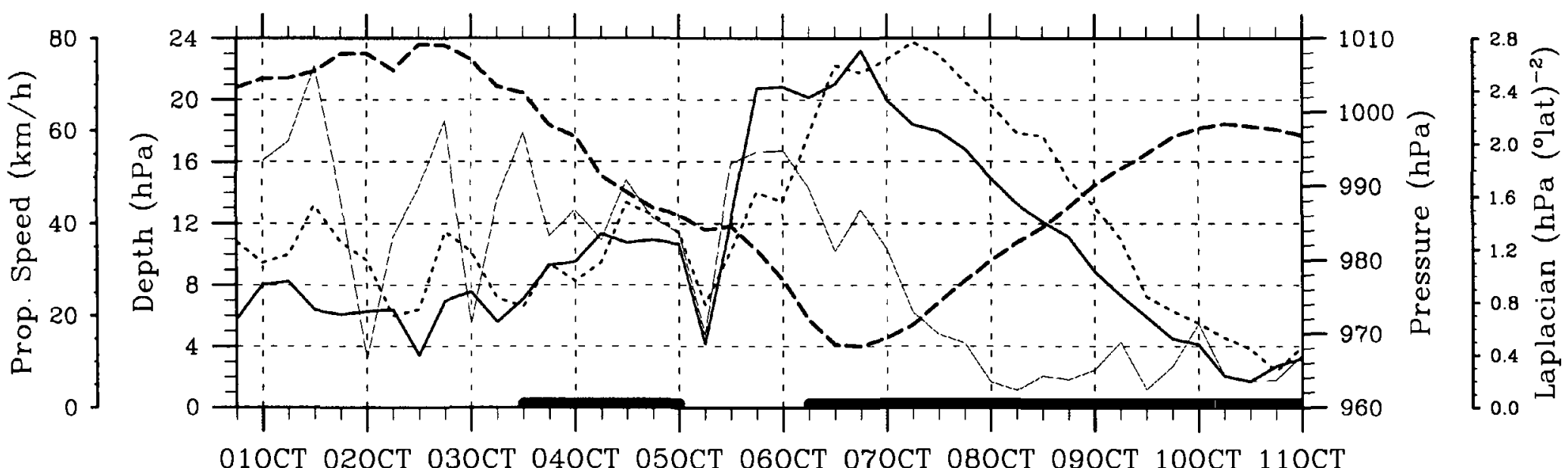

Figure 6.3 - Life history of the Nome storm

The life history of the September-October 1992 storm calculated using the Melbourne algorithm. The storm started on 30 September 1992 at $18 \mathrm{Z}$ through 11 October 1992 at 00Z. The solid line represents the depth of the storm in hPa. The thin solid line is the propagation speed in $\mathrm{km} / \mathrm{h}$. The dashed lines indicate the central pressure of the storm in $\mathrm{hPa}$. The dotted line shows the Laplacian of the pressure field given in $\mathrm{hPa}\left({ }^{\circ} \mathrm{lat}\right)^{-2}$. The spacing in the $\mathrm{x}$-axis is for every 6 hours, starting at $00 \mathrm{Z}$ for each day. The thick lines on the $\mathrm{x}$-axis represent the periods during which the storm center was over the sea. 


\section{Chapter 7 Conclusion}

The overarching topic for the work presented in this dissertation is extratropical storm activity in the regions of the North Pacific and Alaska. Specific focus has been directed towards aspects within this topic that include: improving our knowledge and understanding of climatological aspects of aggregate storm activity, how storm activity is influenced by major climatic drivers, and the performance of specific tools used to give us information about storms. In conducting this work three major storm tracking algorithms (Ch 3) were employed, data from the NCEP/NCAR Reanalysis was utilized, and dedicated runs on the ECHAM 5 model were performed. Table 7.1 summarizes the main dissertation results.

The work undertaken herein was guided by an underpinning research hypothesis: Storm activity in the North Pacific, Bering Sea, and Alaska regions has undergone demonstrable change throughout the past decades, activity that is in part attributable to certain features of the physical environment, such as sea-ice variability, and which also can be a function of the tools utilized in the analysis of storms - the tracking algorithms. Conducting this work required that additional baseline information about storms be established for this region, that storm response to major forcers be examined, and that storm tracking tools be assessed. Thus to test this hypothesis, three specific questions were formulated and addressed, listed next. 


\subsection{Research Question 1}

What are there characteristics and variability of storm tracks in the North Pacific region and how have they changed?

The study presented in Chapter 4 has used the tracking algorithm by Hodges (1995) and the NCEP/NCAR Reanalysis dataset (Kalnay et al. 1996; Kistler et al. 2001) to study the characteristics and variability of storm tracks in the North Pacific. The study region was subdivided into three zones (Gulf of Alaska, Bering Sea, Alaska); for each area a full analysis was conducted for all four seasons, here defined as: winter (December, January and February), spring (March, April and May), summer (June, July and August) and autumn (September, October and November).

Winter emerged as the most active season for most of the storm parameters, including genesis density and track density. Winter storm tracks are also located farther south, a result consistent with other studies (Simmonds; Keay 2002).

The lysis density analysis results reinforce the concept that the Gulf of Alaska is the "graveyard" of storms in the North Pacific as pointed out, for example, by Simmonds and Keay (2002). The track density results suggest that most of the storm systems that end up in the Gulf of Alaska do not form locally. A targeted assessment verified this fact, which indicates that most storms are not forming locally. An exception to this was the summer season, for which a source region in the Aleutian Islands was indicated. There are indications of tropical cyclones undergoing extratropical transitions and reaching the Gulf of Alaska, especially during summer and autumn, as these systems may have enough energy to move to far distances. Once in the Gulf of Alaska, there are several mechanisms at work that contribute to the large observed lysis values: barotropic processes and the regional topography. The regional 
topography in Alaska may act to block the low-level moisture of a storm system (Pickart et al. 2009) and inhibiting further progression of the system, in this case dying in the GOA region.

As for the lifetime, summer storms last longer than any other season. This result expands upon two previous studies (Mesquita et al. 2008; Zhang et al. 2004) that confined comparisons of lifetime to summer and winter. Summer storms are also the least intense, with winter, spring and autumn intensities being greater and similar to one another. The mean speed of the storms is higher in winter. In summary, summer storms last longer, move more slowly and are less intense. One important feature of the climatology analysis is that the transitional seasons, spring and autumn, tended to have similar values to each other for most of the storm parameters considered.

Of the three aggregate regions considered in this paper, the Alaska Interior/Yukon has higher annual genesis density compared to the other regions. The Gulf of Alaska has higher number of storms (track density) and higher lysis density. The Bering Sea shows storms with higher lifetime, intensity and speed.

In general, most of the storm parameter trends observed in this study were not very robust. Most of the statistically significant trends at the $99 \%$ s.l. are related to the genesis density for the Alaska Interior/Yukon region, and genesis density and lysis density for the Bering Sea.

When considering the trends for the three regions and the three different periods of time - the entire 1948-2008 timeseries and pre- and post 1979 - we observe that most of the trends that are statistically significant (at the $95 \%$ and $99 \%$ significance levels) occur in the Gulf of Alaska region, generally for genesis density 
and lysis density. Two variables are of particular importance for Alaska when it concerns trends are: the number of storms (track density or storms entering a region) and their intensity - none of the three regions considered show trends for these variables. An interesting result is a clear indication that SST does not appear to be a strong storm control for the marine regions explicitly considered: Gulf of Alaska and Bering Sea. Instead, local shear and topography conditions appear to exert the greater control, by extracting energy from a storm system leading to its decay.

\subsection{Research Question 2}

What is the role played by the Sea of Okhotsk sea-ice anomalies on storm activity?

The relationship between Northern Hemisphere extra-tropical storm activity and sea-ice cover over the Sea of Okhotsk were examined in Chapter 5. An index based on sea-ice concentration in the Sea of Okhotsk region was created, called the SOKc index (See Table 7.2). There are different mechanisms at play in that region: sea-ice cover over the Sea of Okhotsk is influenced by changes in the storm tracks associated with the large-scale circulation, and in turn, the changes in sea-ice cover can impact the large-scale circulation (Honda et al. 1999).

Results show how much the sea-ice cover over the Sea of Okhotsk may affect most storm track variables, not only in the North Pacific, but also in the Atlantic. A mechanism for that could be the seeding of the Pacific storms in the Atlantic. The Atlantic changes confirm the NAO damping suggested by Yamamoto et al. (2006), although it must be pointed out that, in a modeling study they performed using an AGCM, when they limited anomaly changes to the Sea of Okhotsk, the damping of the North Atlantic Oscillation (NAO) signal was not confirmed. This could have been 
a byproduct of their model simulation. Our modeling simulations using the idea of 'persistence' confirmed the NAO damping.

Changes in SOKc also help explain some of the variability in the Gulf of Alaska region. If local SST is not influencing the Gulf of Alaska storm activity (Mesquita et al. 2009), then a plausible explanation would be that the influences of large-scale atmospheric variability influence on the sea-ice cover in the Sea of Okhtosk, which in turn influence regional storm activity.

ECHAM5 runs show that sea-ice persistence (winters with similar sea-ice concentration in the Sea of Okhotsk) may play an important role in the damping of the NAO. Appendix D shows more details about the ECHAM5 model.

\subsection{Research Question 3}

How important is the choice of a tracking algorithm and what are some of the synoptic/dynamic influences on a typical strong storm event in the North Pacific?

The choice of a tracking algorithm that may account for more accurate cyclogenesis and cyclolysis estimates is very important. The third paper in this dissertation (Chapter 6) showed that a system undergoing mid-life cycle reenergization was counted as two systems by the NOAA "GS" algorithm (Serreze 1995). However, the same system was then counted as one system by the Melbourne algorithm (Murray; Simmonds 1991). The Melbourne algorithm thus reduces the chances of overcounting. The cyclogenesis and cyclolysis locations for this storm were also tracked more accurately by the Melbourne algorithm.

The tracking gap, situated over the Chukotka region in Russia at $18 \mathrm{Z} 05$ Oct. 1992, was likely triggered in the GS algorithm by a temporary weakening of the 
system. By looking at the Melbourne algorithm data around that time (Table 7.3), we observe that the storm track became a strong open system on 05 October at $12 \mathrm{Z}$, and then it closed again at $18 \mathrm{Z}$. The main reason the storm weakened to an open system was due to the influence of a high-pressure system located along Alaska, the Aleutian Islands and part of the North Pacific Ocean in a SW-NE axis. The low-pressure system was "guided" by this blocking high system until 05 October at $12 \mathrm{Z}$ when this large blocking high was split into two along the Aleutian Islands. This caused the low-pressure system over Russia to become spatially larger, to strengthen and to start opening. From 05 October at $00 \mathrm{Z}$ to 06 October at $00 \mathrm{Z}$, the radius of the pressure system had increased by $108 \%$ (Table 7.3 ).

These results have also shown that the concept of a "storm" and how this concept is treated in tracking algorithms may affect their tracking results. It should be noted that an algorithm should be applied according the users' needs and each tracking algorithm has its strengths and weaknesses, so great care must be taken not to draw misleading conclusions (Leonard et al. 1999). For example, if one desires only a count of tracks passing a given location, then concerns about double counting issues with cyclogenesis and cyclolysis locations issues might not be important.

This study has also shown the important synoptic/dynamic role played by the presence of a blocking high system over the Gulf of Alaska. It helped shape the Nome storm of 1992 and it seems to be an important constituent for storms that cause flooding in Nome (Blier et al. 1997; Fathauer 1978). In the North Pacific, this blocking high serves to: a) interact with an approaching low to develop a relatively steep pressure gradient along the ridge west side; b) entrain warmth and moisture in 
the ensuing strong southerly flow and advect it into the low; and to c) deflect these now revitalized storms to the northeast, towards the Aleutian Islands and Bering Sea.

In addition to these synoptic factors, the re-energization of the 1992 Nome storm could also be explained by broader dynamic factors, including a transfer of kinetic energy from the background flow associated with the ridge break up process, and the increased local availability of water vapour as it traversed the Sea of Okhotsk and the Gulf of Anadyrsk.

The energetics of the Nome storm were also studied in a follow-up paper (See Appendix C).

\subsection{Final Discussion}

Most of the results found in our studies show that storm variables have exhibited change since 1979 in the North Pacific. However, one exception is the intensity variable, which has not shown much change in the Bering Sea, Gulf of Alaska and Interior Alaska/Yukon. The blocking high has also been shown to be of great importance in extreme events in Alaska, when storm systems are deviated towards Kamchatka. These blocking systems have also been recently associated with influencing a reduction in upwelling in the Alaskan Beaufort Sea (Pickart et al. 2009).

Sea-ice over the Sea of Okhotsk was also shown to influence the storm track activity in the North Pacific and thus influencing the Atlantic as well. These changes may point to the fact that, under a global warming scenario where less sea-ice could be found in the Sea of Okhotsk, changes to the storm tracks in the North Pacific could be expected as well. The linear combination of the storm tracks and different 
teleconnections patterns has also pointed out the importance of patterns such as the North Atlantic Oscillation and the West Pacific.

It should be noted that one of the caveats of the dissertation is related to the methodology chosen. Pickart et al. (2009) used manual tracking in his study because of the different interactions between neighboring storms in the Alaskan region, but the authors also point out that in future work will address the fidelity by which an automated procedure can reproduce their results. This dissertation has shown that certain algorithms may be close to manual tracking.

Cyclone statistics from a Lagrangian perspective may depend on the tracking scheme. Similarly, results from an Eulerian analysis will depend on the method used, e.g. band pass filtered variance can be performed with a Lanczos filter or a Fourier transform filter and different researchers choose different bands.

However, the cyclone statistics probably depend more on what choice of field is used to identify the cyclones. This issue is discussed in Bengtsson et al. (2006), who argue that this is the case, especially when using Mean Sea Level Pressure (MSLP). MSLP may reflect changes in the large-scale background pressure field, so that identification of cyclones in the raw MSLP may actually reflect these changes rather than changes in the storm intensities. Bengtsson et al. (2006) also mention, on their page 19 , that this "is likely to be less prevalent in methods that use the Laplacian of MSLP to identify the cyclones or that use of a completely different field that is less influenced by the large-scale background, such as relative vorticity".

In the dissertation results, storm track trends were not robust in the North Pacific. As mentioned before, this could be a result of the choice of field used. Bengtsson et al. (2006) also used the tracking algorithm by Hodges to study storms in 
a climate change context. They found an invariant property of storms - no indication of an increase in intensity, nor in the number of extratropical storms. Their study and ours contrast to previous studies that indicated an increase in intensity or number of storms. These previous studies relied mostly on tracking storms based on MSLP or surface geopotential height, where an increase in storm intensity is observed in global domains (Bengtsson et al. 2006). Thus the observed trends in previous studies may be a reflection of changes in the large-scale background pressure filed, and not in the individual storm systems per se. 
Table 7.1 - Main dissertation results

The five main dissertation results from each of the research papers.

\section{Research question 1 - Journal of Climate paper}

$1 \quad$ Storm track inter-seasonal variability is not large in spring and autumn

2 North-south shift of the main track maxima throughout the seasons with a rotation to the northeast

3 Barotropic processes and blocking influence of the coastal orography of northwest North America helped shape the downstream end of the storm tracks in the North Pacific. Storms that die in the Gulf of Alaska do not form locally and there are indications of tropical systems undergoing extra-tropical transition before reaching the Gulf of Alaska

$4 \quad$ Summer storms are longest in duration compared to the other seasons

5 Temporal trends tended to be weak over the study area. And the sea surface temperature did not emerge as a major cyclogenesis control in the Gulf of Alaska.

\section{Research question 2 - Tellus A paper - submitted}

1 Sea ice cover over the Sea of Okhotsk affects storm track variables, not only in the North Pacific, but also in the Atlantic

2 The Atlantic changes confirm the NAO damping

3 Seeding from the Pacific storms into the Atlantic help change the Atlantic eddy-driven jet, thus altering the transient eddy vorticity fluxes - and it is the low-frequency vorticity flux and divergence that contribute toward the NAO decay The ECHAM5 simulations demonstrated the idea of persistence, where similar sea-ice conditions in the Sea of Okhotsk region could provide a significant effect on the $N A O$

5 Changes of sea ice in the Sea of Okhotsk affect the intensity of the storms, especially on the west side of the North Pacific towards the Bering Sea where systems traveling in that direction are known to cause flooding in Alaska (e.g.: the Nome storm of October 1992)

\section{Research question 3-Geophysical Research Letters paper}

$1 \quad$ Both the NOAA and the Melbourne algorithms captured the essential features of the Nome storm, but they differ in the genesis and lysis location. The NOAA algorithm broke the storm into two separate events affected how the tracking algorithms handled the event

3 The blocking high serves to interact with an approaching low to develop a steep pressure gradient, entrain warmth and moisture into the low and to deflect the revitalized storms to the northeast The storm reenergization may have been facilitated by a drawing of kinetic energy from the background flow associated with the ridge break up process The Melbourne algorithm provides additional insights into the storm evolution, in terms of genesis location and longevity, via the description of open and closed centers, as well as variables like Laplacian and depth 
Table 7.2 - SOKc index

The monthly standardized Sea of Okhotsk Index from 1978(9) to 2007.

\begin{tabular}{c|rrr} 
Year & DEC & \multicolumn{1}{c}{ JAN } & FEB \\
\hline 1979 & 0.7883 & 2.7296 & 2.0079 \\
1980 & 1.2443 & 1.0988 & 1.2715 \\
1981 & -0.3217 & -0.4378 & 0.4322 \\
1982 & -0.6474 & 0.3667 & 0.4989 \\
1983 & 1.7910 & 0.2551 & 1.3086 \\
1984 & -0.5361 & -0.1055 & -1.0010 \\
1985 & 1.2809 & 0.9222 & 0.3690 \\
1986 & -0.2257 & 0.2107 & 0.4419 \\
1987 & -0.1868 & 0.4697 & 0.4190 \\
1988 & -0.7021 & 0.7884 & -0.1886 \\
1989 & -1.0605 & -0.9507 & -0.8517 \\
1990 & 0.0360 & -0.2173 & 0.4370 \\
1991 & -0.7301 & -1.1175 & -1.2817 \\
1992 & -0.7881 & -0.2893 & 0.0834 \\
1993 & 0.8234 & 0.3578 & 0.1537 \\
1994 & 1.0760 & 0.9659 & -0.7266 \\
1995 & 0.5626 & 1.0335 & 0.4480 \\
1996 & -1.1602 & -1.6536 & -1.5420 \\
1997 & -0.5408 & -2.1913 & -2.2323 \\
1998 & -1.8970 & -1.3074 & -1.0402 \\
1999 & 2.1034 & 0.3236 & -0.0520 \\
2000 & -0.8656 & -1.3431 & 0.1340 \\
2001 & 1.8065 & 0.7536 & 1.6108 \\
2002 & 0.1112 & -0.1158 & 0.8957 \\
2003 & -0.1381 & 0.5763 & 0.8205 \\
2004 & 0.1403 & -0.0269 & -0.5378 \\
2005 & 0.0642 & -0.0919 & -0.9676 \\
2006 & -1.3663 & -0.8259 & -1.2191 \\
2007 & 0.0847 & -0.1780 & 0.3086
\end{tabular}


Table 7.3 - Storm parameters from the Melbourne algorithm

Storm parameters of the September-October 1992 storm given by the Melbourne algorithm. The data indicated here is only for the period between 05 September 1992 at $00 Z$ through 07 October 1992 at 18Z. IOP represents the 'index of pressure', that is, the type of low: CS for strong closed low, OS for strong open low, OW for weak open low and CW for weak closed low.

\begin{tabular}{|c|l|c|c|c|c|c|}
\hline Date & \multicolumn{1}{|c|}{ Time } & IOP & $\begin{array}{c}\text { Pressure } \\
\mathbf{( h P a})\end{array}$ & $\begin{array}{c}\text { Laplacian } \\
\mathbf{h P a} \text { ('lat) }\end{array}$ & $\begin{array}{c}\text { Depth } \\
\text { (hPa) }\end{array}$ & $\begin{array}{c}\text { Radius } \\
\text { ('lat) }\end{array}$ \\
\hline $05 / 10 / 92$ & $00 Z$ & CS & 986.19 & 1.33 & 10.69 & 6.99 \\
\hline & $06 Z$ & CS & 984.17 & 0.79 & 4.16 & 4.34 \\
\hline & $12 Z$ & OS & 984.63 & 1.20 & 12.20 & 6.38 \\
\hline & $18 Z$ & CS & 981.37 & 1.63 & 20.74 & 7.90 \\
\hline $06 / 10 / 92$ & $00 Z$ & CS & 977.38 & 1.57 & 20.83 & 9.03 \\
\hline & $06 Z$ & CS & 971.93 & 2.08 & 20.15 & 7.76 \\
\hline & $12 Z$ & CS & 968.54 & 2.60 & 21.02 & 7.20 \\
\hline & $18 Z$ & CS & 968.35 & 2.54 & 23.21 & 7.10 \\
\hline $07 / 10 / 92$ & $00 Z$ & CS & 969.46 & 2.64 & 19.96 & 6.72 \\
\hline & $06 Z$ & CS & 971.25 & 2.77 & 18.39 & 6.61 \\
\hline & $12 Z$ & CS & 974.22 & 2.68 & 17.95 & 6.47 \\
\hline & $18 Z$ & CS & 977.25 & 2.47 & 16.81 & 6.97 \\
\hline
\end{tabular}




\section{References}

Bengtsson, L., K.I. Hodges, and E. Roeckner, 2006: Storm tracks and climate change. J. Clim., 19, 3518-3543.

Blier, W., S. Keefe, W. A. Shaffer, and S. C. Kim, 1997: Storm Surges in the Region of Western Alaska. Monthly Weather Review, 125, 3094-3108.

Fathauer, T. F., 1978: A forecast procedure for coastal floods in Alaska. NOAA Tech. Memo., NWS AR-23, 27 pp.

Hodges, K. I., 1995: Feature Tracking on the Unit Sphere. Mon. Wea. Rev., 123, 3458-3465.

Honda, M., K. Yamazaki, H. Nakamura, and K. Takeuchi, 1999: Dynamic and Thermodynamic Characteristics of Atmospheric Response to Anomalous Sea-Ice Extent in the Sea of Okhotsk. J. Climate, 12, 3347-3358.

Kalnay, E., and Coauthors, 1996: The NCEP-NCAR 40-Year Reanalysis Project. Bull. Amer. Meteor. Soc., 77, 437-471.

Kistler, R., and Coauthors, 2001: The NCEP-NCAR 50-year Reanalysis: monthly means CD-ROM and Documentation. B. Am. Meteorol. Soc., 82, 247-267.

Leonard, S. R., J. Turner, and A. V. D. Wal, 1999: An assessment of three automatic depression tracking schemes. Meteorological Applications, 6, 173-183.

Mesquita, M. d. S., D. E. Atkinson, and K. I. Hodges, 2009: Characteristics and Variability of Storm tracks in the North Pacific, Bering Sea and Alaska. J. Climate, In press.

Mesquita, M. D. S., N. G. Kvamstø, A. Sorteberg, and D. E. Atkinson, 2008: Climatological properties of summertime extra-tropical storm tracks in the Northern Hemisphere. Tellus $A, 60$.

Murray, R., and I. Simmonds, 1991: A Numerical Scheme for Tracking Cyclone Centres from Digital Data. Part I: Development and Operation of the Scheme. Aust. Met. Mag., 39, 155-166. 
Pickart, R. S., G. W. K. Moore, D. J. Torres, P. S. Fratantoni, R. A. Goldsmith, and J. Yang, 2009: Upwelling on the continental slope of the Alaskan Beaufort Sea: Storms, ice, and oceanographic response. J. Geophys. Res., 114.

Serreze, M. C., 1995: Climatological Aspects of Cyclone Development and Decay in the Arctic. Atmos-Ocean, 33, 1-23.

Simmonds, I., and K. Keay, 2002: Surface Fluxes of Momentum and Mechanical Energy Over the North Pacific and North Atlantic Oceans. Meteor. Atmos. Phys., 80, $1-18$.

Yamamoto, K., Y. Tachibana, M. Honda, and J. Ukita, 2006: Intra-seasonal relationship between the Northern Hemisphere sea ice variability and the North Atlantic Oscillation. Geophys. Res. Lett., 33, L14711.

Zhang, X., J. E. Walsh, J. Zhang, U. S. Bhatt, and M. Ikeda, 2004: Climatology and Interannual Variability of Arctic Cyclone Activity: 1948-2002. J. Climate, 17, 23002317. 


\section{Appendix A}

\section{Appendix A - Contributions to the Dissertation Chapters}

\section{A.1 Chapter 4}

The tracking algorithm and the input files were provided by Dr. Kevin I. Hodges. The literature search, writing, installation of the tracking algorithm, running the tracking algorithm, preparation of figures and the work of corresponding author were conducted by Michel d. S. Mesquita. Text re-editing and guidance were provided by Dr. David E. Atkinson.

\section{A.2 Chapter 5}

The tracking algorithm, input files, guidance and physical interpretations were provided by Dr. Kevin I. Hodges. The literature search, writing, running the tracking algorithm and the preparation of figures were conducted by Michel d. S. Mesquita. Dr. Jüergen Bader made the ECHAM5 runs and Figure 5.10. Dr. Atkinson provided the text re-editing and guidance.

\section{A.3 Chapter 6}

Dr. Ian Simmonds and Kevin Keay provided the Melbourne tracking algorithm and guidance. They also provided the facilities at the University of Melbourne (Australia) during my visit to their department in January 2009. The literature search, writing, running the tracking algorithm, preparation of figures, preparation of the manual tracking software (Appendix B) and the work of corresponding author were conducted by Michel d. S. Mesquita. Michel d. S. Mesquita also wrote the proposal 
submitted to the proposal competition organized by the Center for Global Change at UAF. The proposal was one of the selected winners and the funding allowed Mesquita to visit the Earth Sciences Department at the University of Melbourne. Dr. David E. Atkinson provided the text re-editing and guidance. The data from Serreze's algorithm were provided by Dr. David E. Atkinson and Jon Gottschalck. 


\section{Appendix B}

\section{Appendix B - Manual Storm Tracking}

\section{B.1 File Description}

The following GRADS program was written by Mesquita to aid the manual tracking of a storm. Any variable may be used. The program is interactive: as the user clicks on a point on the map (e.g.: a storm center), the program records the latitude and longitude of the point and advances one timestep. After the tracking is finished, it plots the entire track and a file is produced with the exact latitude and longitude values.

\section{B.2 File: storm_traj.gs}

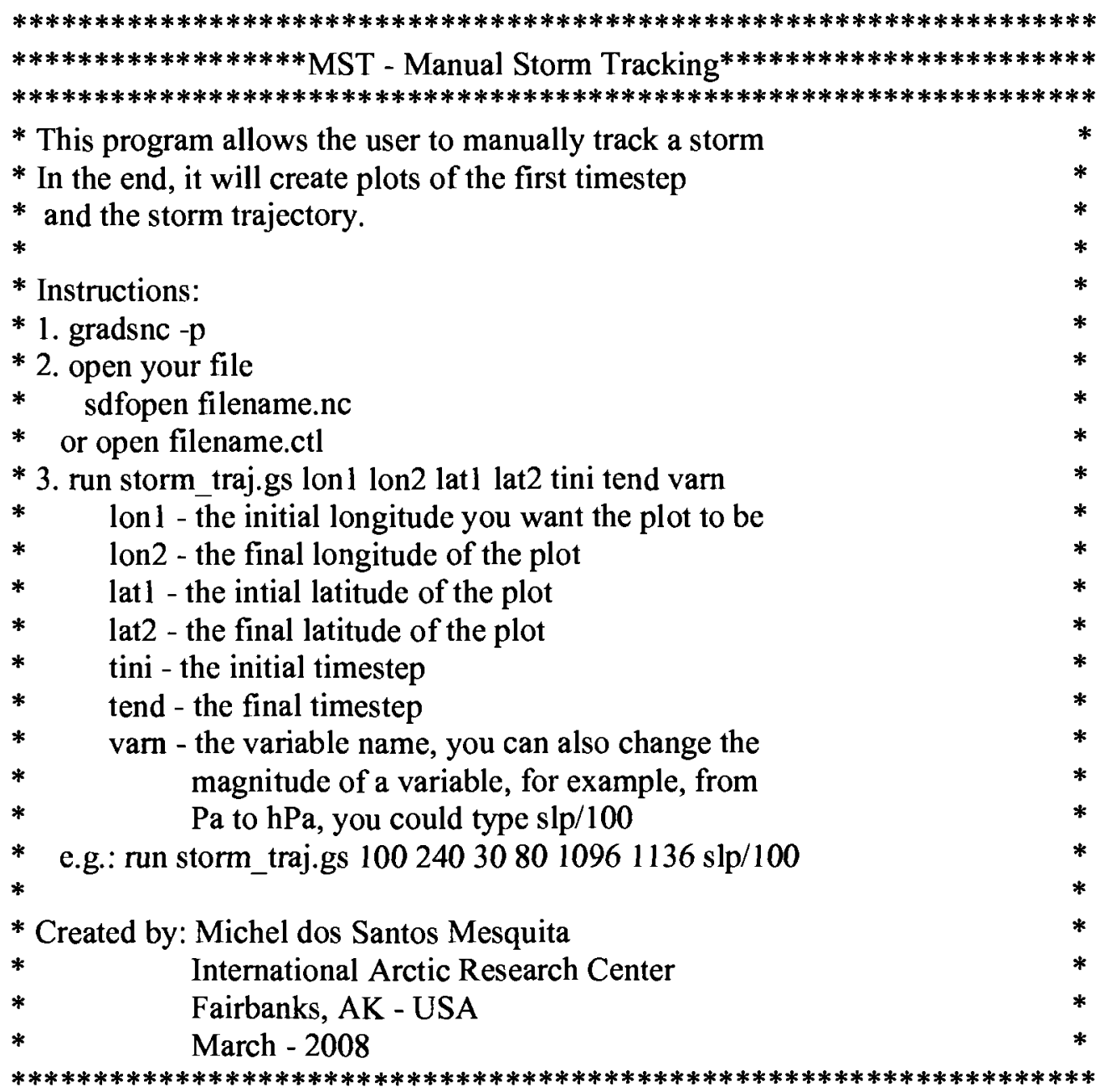


function main(arg)

lol $=$ subwrd $(\arg , 1)$

lo2=subwrd(arg,2)

la $1=$ subwrd(arg,3)

la2=subwrd $(\arg , 4)$

tini $=$ subwrd $(\arg , 5)$

tend=subwrd(arg,6)

$\operatorname{varn}=$ subwrd $(\arg , 7)$

* Set the lat and lon based on the arguments passed

'set lon ' lo1 ' ' lo2

'set lat ' la1 ' ' la2

* Define the thickness and color of the track line

'set line 216 '

'set grads off

* Number of timesteps

tdiff $=$ tend-tini

tempo $=0$

$\mathrm{t} l=$ tini

while (tempo $<$ tdiff)

* Initial time step

'set $t$ 'tini

* Contour spacing to make it easier to track the center of the low

* change this to suit your case study

'set cint 1 '

* Display variable

'd 'varn

*'run click.gs 'tempo' 1'

itempo=tempo

flag=1

say itempo

say flag

if (flag=1)

say 'Click on the map, for example, the center of a low...'

say ' ' 
* This command allows the user to click on the GrADS screen 'q pos'

* Get the first (x) and second (y) coordinates of the point $\mathrm{x}=$ subwrd(result,3)

$\mathrm{y}=$ subwrd(result, 4 )

* Convert the $\mathrm{x}$ and $\mathrm{y}$ to lon and lat coordinates 'q $x y 2 w$ ' $x$ ' 'y

* Get the results from the query and assign to variables

* This is only if you are interested in the LAT and LON

* Position of where you are clicking

LONG=subwrd(result,3)

LATI=subwrd(result,6)

say "Longitude: "LONG

say "Latitude: "LATI

* Define the variables in GrADS ( $x$ and $y$ )

'define $x$ 'itempo' $=$ ' $x$

'define y'itempo'='y

'q define'

endif

'c'

tini $=$ tinit 1

tempo $=$ tempo +1

endwhile

* Display the first timestep

* If you want, change this part to show the trajectory

* with the desired timestep

'set $t$ ' $t$ 1

'set grads off'

'd 'varn

'enable print storm_traj.gm'

* The following lines are due to the fact that the command

* draw line does not accept variables for numbers

tempo $=1$

while $($ tempo $<=($ tdiff-1) $)$ 
'd x'tempo-1

$\mathrm{xxl}=$ subwrd(result,4)

say "xxl is: " $\mathrm{xx} 1$

'd y'tempo-1

yyl=subwrd(result,4)

'd x'tempo

$\mathrm{x} \times 2=$ subwrd(result, 4$)$

'd y'tempo

yy $2=$ subwrd(result,4)

* write to a file, without appending

'set grads off'

write(result.gs,"'draw line " xx1 " " yy1 " " xx2 " " yy2 "'")

close(result.gs)

* run the file to plot the line segment

'run ./result.gs'

*COMMENT: draw line MUST take numbers not variables for numbers, so the following line won't work.

*'draw line $x^{\prime}$ tempo-1' $y$ 'tempo- l' $x$ 'tempo' $y^{\prime}$ tempo

tempo $=$ tempo +1

endwhile

'print'

'disable print'

'!echo"**************MAKING FIGURE******************************"'

"echo "Converting and creating figures: "'

'!gxeps -ci storm_traj.gm -o storm_traj.eps'

'gxeps -i storm_traj.gm -o storm_traj_bw.eps'

"echo "Deleting storm_traj.gm"'

'!rm storm_traj.gm'

"!echo "Converting to PNG"

"'convert -density 300 storm_traj.eps storm_traj.png'

'!convert -density 300 storm_traj_bw.eps storm_traj_bw.png'

"echo "Four figures were saved: EPS and PNG format (color and BW)"'

"echo "Thank you for using MST: Michel Mesquita - mmeclimate@gmail.com"'

"echo

" *************************END 


\section{Appendix C}

\section{Appendix C - Nome Storm Follow-up: Paper Collaboration}

\section{C.1 Work Description}

The following paper was a follow-up work to the Nome paper presented in Chapter 6 . The idea was to analyze the storm energetics of that system. This was a collaboration work that started while I was visiting the University of Melbourne in January 2009. It involves the groups from the University of Melbourne in Australia (Dr. Alexandre Pezza, Dr. Ian Simmonds and Kevin Keay), from the Amazonas State University in Brazil (Dr. José Augusto Paixão Veiga) and myself (Michel dos Santos Mesquita). 


\title{
C.2 Environmental Energetics of an Exceptional High-latitude Storm ${ }^{4}$
}

\begin{abstract}
This study presents the Lorenz energetics of an exceptional baroclinic storm propagating to Nome (Alaska) in October 1992, where it caused severe flooding. The storm track was greatly influenced by the interaction with a blocking high a week before the cyclone was formed. The energetics gives new insights into this dramatic storm, suggesting that the large scale environment was responsible for its long trajectory and intensification. The blocking high also provided the dynamic steering that facilitated the wave propagation and baroclinic growth. Our results show that the environmental energy transfers can be used as an informative metric for severe storms.
\end{abstract}

\footnotetext{
${ }^{4}$ Pezza, A.B., J.A.P. Veiga, I. Simmonds, K. Keay and M.d.S. Mesquita, 2009: Environmental energetics of an exceptional high-latitude storm. Atmos. Sci. Lett., accepted.
} 


\section{C.2.1. Introduction}

High latitude severe storms have been increasingly subject to scrutiny as they are an important indicator of changes in the global climate, particularly in the Northern Hemisphere (NH) where large continental air masses lie close to the pole (Eichler and Higgins, 2006). These intense cyclones are influenced by changes in robust modes of circulation (e.g. the annular modes) (Hall and Visbeck, 2002), changes in sea ice (Stroeve et al., 2007; Comiso and Nishio, 2008; Simmonds et al., 2008), meridional heat and momentum transfers (Uotila et al., 2009) and precipitation anomalies (Hurrell, 1995; Serreze et al, 1997; Renwick, 2002). Under realistic scenarios of climate prediction the baroclinic zones of both hemispheres shift poleward, while mid-latitude blocking-like ridges become more prominent (Herweijer and Seager, 2008). As a result of this synergy, many countries may receive less rain whereas others are expected to experience more frequent cyclone development, especially in high latitudes in the $\mathrm{NH}$, posing a threat to life and property.

Extratropical cyclones have been the subject of studies using the Lorenz energetics in the past with emphasis on understanding the importance of heat and momentum transfers in mesoscale simulations (Smith, 1980; Robertson and Smith, 1983; Michaelides, 1992; Wahab et al., 2002; Marquet, 2003; Decker and Martin, 2005). Recently the technique was used to study the tropical transition of the first known South Atlantic hurricane (Pezza and Simmonds, 2005) from a large scale energy-transfer perspective (Veiga et al., 2008). This was the first time that a dynamic transition (from cold to warm core) of a storm was explained entirely based on environmental energetics, opening a new avenue of exploration of extreme 
weather events and their connection with climate. These recent results demonstrated that the baroclinic and barotropic conversions occurring in the large scale environment had a fundamental role in establishing the right conditions for the transition (Veiga et al., 2008). The energy transfer is believed to occur indirectly from the environment to the vortex so that once the vortex has been initiated local processes (e.g. thermodynamics) may become more important, but we note that the eddies can also provide energy to the background. In the subtropics, the presence of a persistent blocking can facilitate a greater rate of growth when the thermodynamics over the ocean is favorable, but very little is known of how these interactions come about at high latitudes.

We here advance further on this topic and explore the energetics of a very intense high latitude NH system observed in October 1992. Mesquita et al. (2009) provide a preliminary documentation of this storm, showing that it initially formed in eastern China and exhibited its greatest rate of intensification as it approached the Bering Sea. Although only light rain and snow were recorded in Nome, the storm caused unprecedented flooding as a result of the optimum wind fetch. The storm had minimum pressure of $962 \mathrm{hPa}$ on $6^{\text {th }}$ October with near hurricane-force winds causing over $\$ 6$ million dollars in damage. This system is of significant interest also because of its marked latitudinal displacement of about $30^{\circ}$ in seven days, following a nearconstant rate of latitude increase facilitated by a blocking system over the northwest Pacific. As blocking frequency and location is expected to experience significant shifts under global warming scenarios there is a strong potential for significant changes in the behavior of high latitude storms. This paper is a first step in elucidating how well the Lorenz energetics can portray fundamental changes in the 
lifecycle of severe storms, opening new avenues for climate change studies of storm behavior. Data and Methodology are discussed in section 2 and the main results are presented in section 3. A follow up discussion is presented in section 4, commenting on new insights and perspectives brought by the results.

\section{C.2.2. Data and Methodology}

A traditional and compact form of presenting energy computations is suggested by Lorenz (1967), where the available potential energy can be portioned into zonal available potential energy (AZ) and eddy available potential energy (AE). By analogy, the kinetic energy can also be portioned into zonal (KZ) and eddy (KE) components. In the energy cycle of Lorenz, $\mathrm{AZ}$ and $\mathrm{AE}$ are produced by generation processes named GZ and GE (generation of $A Z$ and $A E$ ), while energy terms are connected by conversion terms. Following Muench (1965) the energy budget equations for an open atmospheric can be given as:

$$
\begin{aligned}
& \frac{\partial K Z}{\partial t}=C K+C Z+B K Z+B \phi Z-D Z \\
& \frac{\partial K E}{\partial t}=C E+C K+B K E+B \phi E-D E \\
& \frac{\partial A Z}{\partial t}=-C Z-C A+B A Z+R G Z \\
& \frac{\partial A E}{\partial t}=C A-C E+B A E+R G E
\end{aligned}
$$


In an open area the budget is complicated by the inclusion of nonzero boundary transports. To account for these we include four new components representing the transport of $\mathrm{AZ}, \mathrm{AE}, \mathrm{KZ}$ and $\mathrm{KE}(\mathrm{BAZ}, \mathrm{BAE}, \mathrm{BKZ}$ and $\mathrm{BKE}$, respectively). In addition, $\mathrm{KZ}$ and $\mathrm{KE}$ are associated with the work produced at the boundaries (BФZ and $B \Phi E$, respectively). The last terms in C.1 and C.2, namely $D Z$ and DE, represent dissipation processes. Those terms are here combined and computed in the form of residuals (Wahab et al., 2002). In a similar manner, the intrinsic errors resulting from the numerical estimations for the remaining terms in C. 3 and C. 4 are also included as residuals (see Veiga et al. 2008 for further details).

Six hourly data from the NCEP/NCAR reanalysis $\left(2.5^{\circ} \times 2.5^{\circ}\right.$ resolution [Kalnay et al., 1996]) were used for the gridded energy calculations, cyclone trajectory and large scale atmospheric circulation. Anomalies were calculated over the NCEP 30-year reference period of 1968-1996 (Kalnay et al., 1996) based on weekly and daily climatologies. Horizontal integrations were performed over the areas bounded by $120^{\circ} \mathrm{E}$ and $150^{\circ} \mathrm{W}$, and $50^{\circ}$ and $80^{\circ} \mathrm{N}$ ('Nome box' representing the environment), and $120^{\circ} \mathrm{E} / 150^{\circ} \mathrm{W}, 30^{\circ} \mathrm{N} / 50^{\circ} \mathrm{N}$ ('Blocking box' representing the midlatitude block). These domains are large enough to portray the high latitude environment including the Pacific blocking that 'steered' the system's trajectory (Fig. C.1a). These domains form one of the most active high latitude storm genesis regions on the planet, being of immense importance for infrastructure and fisheries development along the countries involved (for instance coastal infrastructure in Japan and salmon in Alaska). Sensitivity tests showed that our results hold if larger areas are used, and that the separation between blocking and the poleward environment is necessary to accurately quantify the associated energy transfers. For simplicity, key 
periods for the analysis of this storm were defined: week-1 as 20-30 September 1992, week 0 as 30 September -10 October and week +1 as $10-20$ October.

\section{C.2.3. Results}

(a) Blocking and large scale

Figure C.la shows the Mean Sea Level Pressure (MSLP) anomaly for week-1. The MSLP anomaly is indicated in shading for areas with magnitude above $2 \mathrm{hPa}$. A number of other features are also displayed, viz., the $850-200 \mathrm{hPa}$ wind shear vector anomaly for week-1 and the $850-500 \mathrm{hPa}$ thickness anomaly (m) averaged for 6 October 1992 (only magnitude greater than $200 \mathrm{~m}$ shown). The trajectory as identified by the Melbourne University automatic tracking scheme (Simmonds et al., 2003) has also been superposed to the map, with some key phases indicated, viz., the beginning and ending points and the point of maximum intensity. The location of the maximum intensity (marked with an ' $x$ ') given by the tracking scheme for the NCEP reanalysis was $968 \mathrm{hPa}$ on October $6^{\text {th }}$ at $18 \mathrm{UTC}$ (figure C.la), being close to the minimum pressure estimated by the Anchorage analysis chart ( $962 \mathrm{hPa}$ at $12 \mathrm{UTC}$ ). The Nome and Blocking boxes are also indicated. This figure gives the large scale panorama from which the storm was formed. The MSLP during week-1 formed a split blocking pattern of which the polar component resembled very closely the trajectory followed by the storm a week later. The Pacific High over midlatitudes can also be seen at about $37^{\circ} \mathrm{N}$ near the dateline. This high is related to the subtropical ridge that is normally found over that area, but it was found to be considerably stronger and further north than normal during week-1. 
The largest positive MSLP anomaly is seen on the poleward side near the Bering Strait. Not only did this area experience intense blocking during week-1 but it also had the largest windshear anomaly to the south which indirectly contributed to steering the storm motion. The thermal wind implication is that the environment was highly baroclinic at mid-levels (the role of environmental baroclinicty is discussed in detail in section C.2.3b). It can be seen that over most of the storm's trajectory the windshear over week-1 closely follows its path, showing that the storm followed the maximum baroclinicity. The period of maximum intensification (average over October $6^{\text {th }}$ ) is given by mid-level thickness anomaly (green contours). This shows a strong response over the storm, corresponding to an environmental warm anomaly of about five degrees at $850 \mathrm{hPa}$.

Figure C.1b shows the MSLP changes from week-1 to week0 plotted for magnitude greater than $4 \mathrm{hPa}$ (shading) and the $500 \mathrm{hPa}$ temperature anomaly (magnitude above $3^{\circ} \mathrm{C}$, contour) for week-1. The fundamental change in the large scale pressure is that the blocking loses its poleward-split component and intensifies further at mid-latitudes. This comes about with pressure changes which have little zonal variability, showing that the changes are not simply a pressure adjustment associated with the vortex. The $500 \mathrm{hPa}$ temperature anomalies show that most of the domain was cold during week-1, including the poleward blocking split discussed in figure C.la. The Pacific High to the south was predominantly barotropic and warmcored (compare Figs. C.la and C.1b). As discussed next, the energy transfers reveal that the blocking projected a strong barotropic mode about five days before the cyclone was formed, and it also provided the dynamic steering that facilitated the wave propagation and baroclinic growth. 
(b) Energetics

Figure C. 2 shows the time series of the (a) energy and (b,c) conversion terms integrated between 925 and $100 \mathrm{hPa}$ for (a) week 0 and (b) week-1 to week +1 for the Nome Box, and (c) week-1 for the blocking box. The different phases of the storm are indicated: cyclogenesis (L1), rapid intensification (L2); maximum intensity (L3) and decay (L4). It is evident from figure C. $2 \mathrm{a}$ that the environment changed from a state of lower energy to a state of higher energy about two days before the cyclone underwent a rapid intensification (between L1 and L2). This transition started with a peak in zonal available potential energy (AZ) followed by peaks in KE, AE and KZ. The overall energy then rapidly declined from the time the cyclone started to lose intensity (L3). Towards the decay phase (L4) the environment reached a state in which $A Z$ was reduced by about $50 \%$ of its initial value. Below we show that the fundamental process that allowed the energy transfer above came about through baroclinic conversion.

Figure C.2b shows that the conversions over a 30 -day period centered in the storm's maximum period of intensification are maximized for the baroclinic terms $\mathrm{CE}$ and CA. The maximum conversion took place on 6th October O0UTC, about 18 hours before the cyclone reached maximum intensity. As discussed above the large scale domain used for the calculations allows for little direct influence from the vortex in the energy conversion terms, similarly to what was noted by Veiga et al. (2008). However, this is not to say that there is no interaction between the environment and the vortex. In fact, our results suggest that the environment played an important role in determining conditions suitable for the vortex to grow. In this sense there is a suggestion that the vortex grew at the expense of the environment 
primarily through baroclinic processes. The peak in baroclinic energy transfer was maximized between 350 and $600 \mathrm{hPa}$ (not shown). This is the ideal elevation for baroclinic growth at high latitudes partially because the static stability tends to be low. As discussed earlier the blocking further south created ideal conditions for the steering of the storm, but it also participated in the energy exchange as seen in figure C.2c. It is evident that a maximum of barotropic conversion occurred on the 25 th September at $18 \mathrm{UTC}$, five days before the cyclone was first identified by the tracking scheme (indicated as PR). Notwithstanding the marked barotropic peak in the blocking box (about $20 \mathrm{Wm}^{-2}$ ) figure C.2c also shows that the other conversion terms were strong during week-1 (magnitude greater than $9 \mathrm{Wm}^{-2}$ ), characterizing an environment favorable for storm development.

The vertically-integrated time-mean energy cycle is illustrated by the Lorenz diagram in figure C.3. Figure C.3a shows the schematic of the Lorenz diagram here adopted, where the arrows indicate the direction of the energy flux (refer to section C.2.2 for further details). Figures C.3b and C.3c show the energy interplays over week-1 and week0 for the Nome box, and figure C.3d shows the energetics for the blocking box over week-1. These results account for an integrated view of what was discussed before, showing that the environment presented strong baroclinic conversions from week-1, intensifying over week0 (CE reaches $4.1 \mathrm{Wm}^{-2}$ ) while the barotropic conversion $\mathrm{CK}$ remains small. However, $\mathrm{CK}$ becomes important for the blocking box (figure C.3c), with similar order of magnitude as the other terms. Compared to the results of Veiga et al. (2008) it is apparent that the energetics of the environment can be confidently used to quantify processes related to extreme 
synoptic development both at the mid and high latitudes, with the blocking being a crucial component of the meridional (north-south) exchanges.

We also note that the generation and boundary fluxes terms are also indicated in the flux diagram. The direct calculation of the boundary pressure work terms pose significant difficulties, particularly due to the magnification of initial errors in the data (Wahab et al., 2002 and references therein). Those terms are here combined with the dissipation terms and estimated as residuals (terms RKZ and RKE indicated in figure C.3a). In a similar manner, the intrinsic errors resulting from the numerical estimations of the other terms in equations C.3 and C.4 are also calculated using the same principle (terms RGZ and RGE indicated in figure C.3a). Figure C.3 reveals that the generation and boundary fluxes are smaller than the energy conversion terms in all cases (panels b-d), indicating that the energetics calculations are robust and above the noise.

\section{C.2.4. Concluding Remarks}

In this paper we analyzed the Lorenz energetics of an extreme high latitude storm calculated over a large scale domain representative of the environment. We found that while a mid-latitude blocking played a fundamental role in the preliminary phases it was the baroclinic energy transfers in the environment that can explain the intense development over the lifecycle. While such an approach does not allow us to directly associate the energy conversions with processes at the scale of the vortex, it conveys key information as to environmental forcing mechanisms, quantitatively demonstrating the environment's participation and the synergy between the blocking, which gave the necessary steering, and the large scale baroclinic growth. Although 
we do not discuss mesoscale details, eddy momentum and heat flux convergence are a likely process in which energy can be transported from the environment into the vortex (Molinari and Vollaro, 1989).

Our results shed new light on two principal fronts: first, the environmental energy transfers can be used as an informative metric for severe storms if calculated over a sufficiently large area; second, the barotropic transfer is important as a precursor, while the baroclinic transfers occurring poleward can explain much of the intensification. These results open new avenues in climate change studies of severe baroclinic storms in that the combined energetics of the blocking and the larger environment have the potential to give an independent measure of storm behavior. This develops further on the recent work of Veiga et al. (2008), suggesting that the Lorenz energetics can be used as a quantitative index of storm activity over high latitudes. In particular, our findings highlight the importance of blocking for downstream cyclone development, suggesting that through the energetics of blocking it is possible to quantify key sensitive information about storm behavior. With high latitude storm occurrence being one of the crucial areas to be affected by climate change it is fundamental that the changes in blocking can also be assessed. While present state-of-the-art climate models do not have enough resolution to resolve all the processes at the vortex level our results strongly suggest that the Lorenz energetics can be used with very good confidence as a proxy for storm activity. This poses the fundamental advantage that the large scale approach intrinsic to this methodology allows it to be easily used with climate model output data. 


\section{Acknowledgements}

ABP and IS would like to thank the Australian Research Council and the Australian Antarctic Science Advisory Committee for funding parts of this work. We are indebted to Petteri Uotila for thoroughly reading an earlier version of this manuscript. 

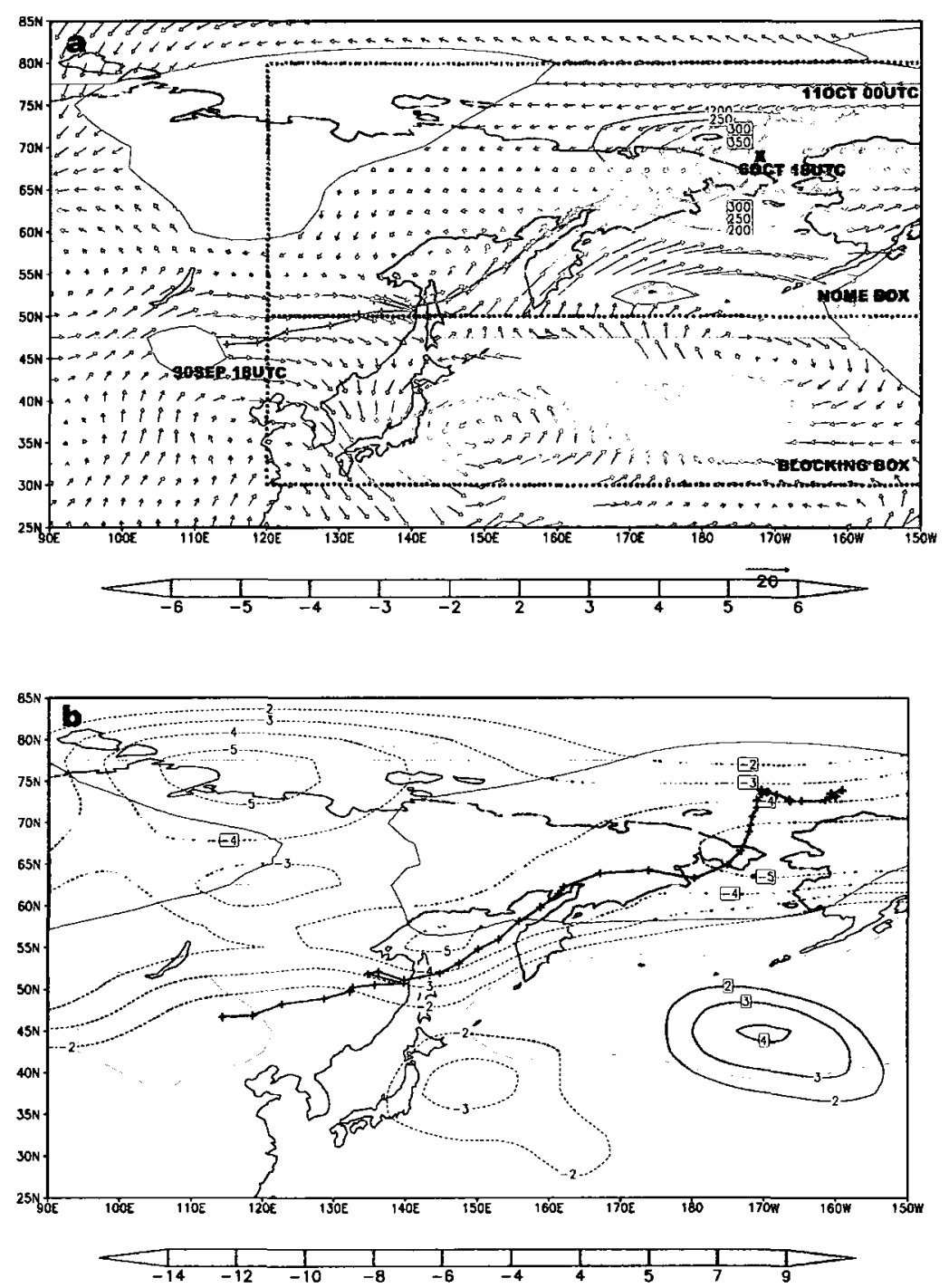

Figure C. 1 - Synoptic plots of the Nome storm

(a) MSLP (magnitude in shading above $2 \mathrm{hPa}$ ) and $850-200 \mathrm{hPa}$ vector windshear anomalies $(\mathrm{m} / \mathrm{s})$ over week-1 and $850-500 \mathrm{hPa}$ thickness anomaly averaged over $6^{\text {th }}$ October (magnitude in contours above $200 \mathrm{~m}$ ); (b) MSLP for week0 minus week-1 (magnitude in shading above $4 \mathrm{hPa}$ ) and $500 \mathrm{hPa}$ temperature anomaly over week-1 (magnitude in contours above $3^{\circ} \mathrm{C}$ ). The cyclone's trajectory is superposed onto $(\mathrm{a} ; \mathrm{b})$ with key dates indicated. The Nome Box and Blocking Box are indicated in (a). Week-1 defined as 20-30 September, Week0 as 30 September - 10 October and Week +1 as $10-20$ October. See text for further details. 

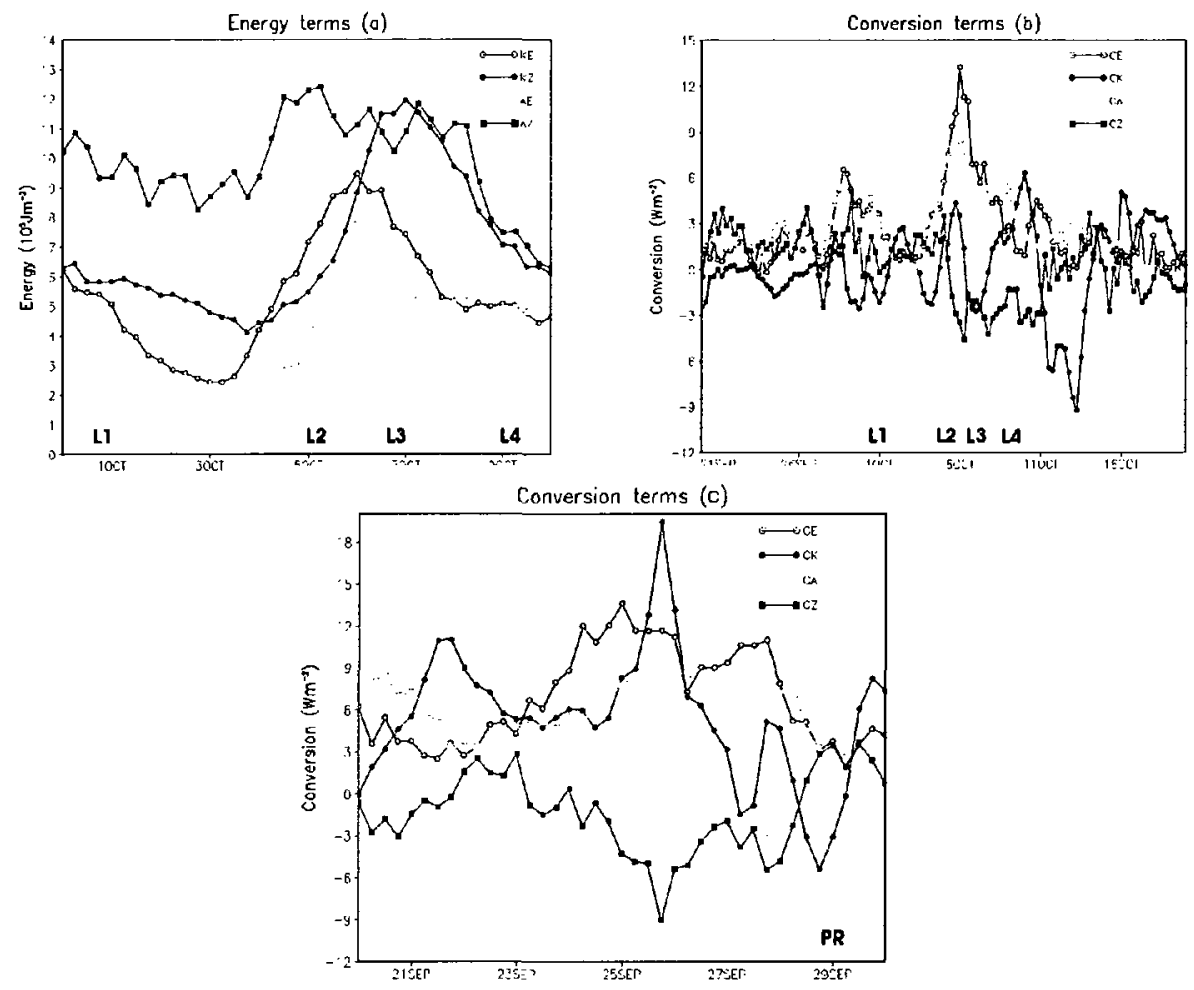

Figure C. 2 - Energy and conversion terms time series

Time series of vertically integrated $(925-100 \mathrm{hPa})$ (a) energy (AE, AZ, KE and KZ) and $(b, c)$ conversion (CA, CE, CK and CZ) terms for (a) week0 for Nome box, (b) from week-1 to week +1 for Nome box and (c) week-1 for Blocking box. The relevant stages during the Nome storm lifecycle are indicated $(\mathrm{Ll}=$ cyclogenesis, $\mathrm{L} 2=$ rapid intensification, $\mathrm{L} 3=$ maximum intensity, $\mathrm{L} 4=$ decay and $\mathrm{PR}=$ preliminary trough). Units are given in (a) $10^{5} \mathrm{~J} / \mathrm{m}^{2}$ and (b,c) W/m . 

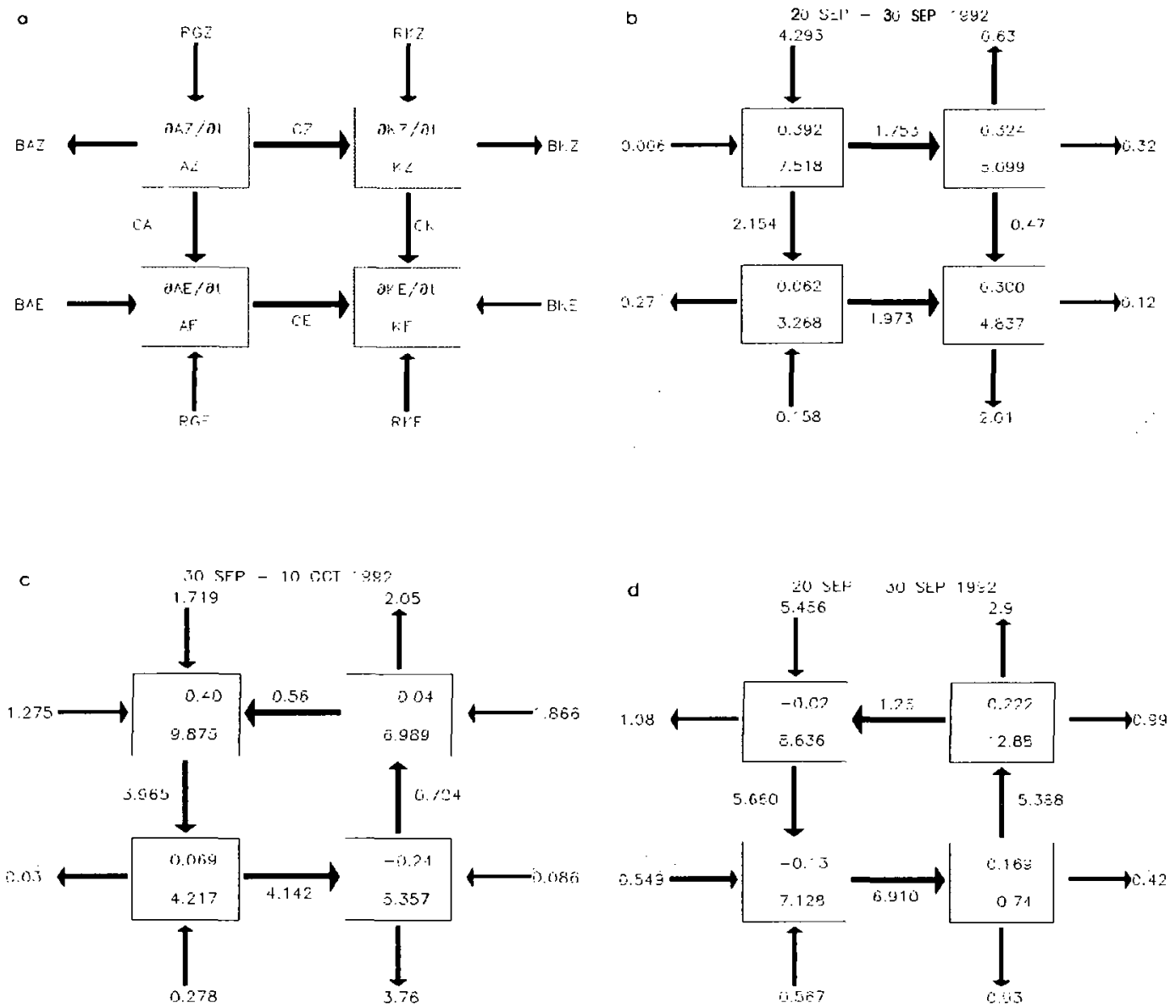

Figure C.3 - Volume integrated energy terms

Volume integrated energy terms (expressed per $\mathrm{m}^{2}$ ) for (b) week- 1 for Nome box, (c) week0 for Nome box and (d) week-1 for Blocking box. Panel (a) shows the energy cycle here adopted as a reference guide. Units are in $10^{5} \mathrm{~J} / \mathrm{m}^{2}$ and $\mathrm{W} / \mathrm{m}^{2}$, respectively, for energy and conversion terms. See text for definition of weeks and further details. 


\section{References}

Comiso, J.C., Nishio F. 2008. Trends in the sea ice cover using enhanced and compatible AMSR-E, SSM/I, and SMMR data, J. Geophys. Res., 113: C02S07, doi: $10.1029 / 2007 \mathrm{C} 004257$.

Decker S. G., Martin J. E. 2005. A local energetic analysis of the life cycle differences between consecutive, explosive deepening, continental cyclones. Mon. Wea. Rev., 133: 295-316.

Eichler T., Higgins W. 2006. Climatology and ENSO-related variability of North American extratropical cyclone activity, J. Clim, 19: 2076-2093.

Hall A., Visbeck M. 2002. Synchronous Variability in the Southern Hemisphere Atmosphere, Sea Ice, and Ocean Resulting from the Annular Mode, Journal of Climate, 15: 3043-3057.

Herweijer C., Seager R. 2008. The global footprint of persistent extra-tropical drought in the instrumental era. International Journal of Climatology, DOI:10.1002/joc.1590, 28: 1761-1774.

Hurrell J. W. 1995. Decadal trends in the North Atlantic Oscillation region temperatures and precipitation, Science, 269: 676-679.

Kalnay E. et al. 1996. The NCEP/NCAR 40-year reanalysis project. Bulletin of the American Meteorological Society, 77: 437-471.

Lorenz E. N. 1967. The Nature and Theory of the General Circulation of the Atmosphere. World Meteorological Organization, $161 \mathrm{pp}$.

Marquet P. 2003. The available-enthalpy cycle. II: Applications to idealized baroclinic waves. Q. J. R. Meteorol. Soc., 129: 2467-2494.

Mesquita M.d.S., Atkinson D.E., Simmonds I., Keay K., Gottschalck J. 2009. New perspectives on the synoptic development of the severe October 1992 Nome storm. Geophys. Res. Lett., 36: L13808, doi:10.1029/2009GL038824.

Michaelides S. C. 1992. A spatial and temporal energetics analysis of a baroclinic disturbance in the Mediterranean. Mon. Wea. Rev., 120: 1224-1243. 
Molinari J., Vollaro D. 1989. External influences on hurricane intensity. Part I: outflow layer eddy angular momentum fluxes, J. Atmos. Sci., 46: 1093-1 105.

Muench H. S. 1965. On the dynamics of the wintertime stratosphere circulation. $J$. Atmos. Sci., 22: 349-360.

Pezza A.B., Simmonds I. 2005. The first South Atlantic hurricane: Unprecedented blocking, low shear and climate change. Geophys. Res. Lett., 32: L15712, doi: 10.1029/2005GL023390.

Renwick J. A. 2002. Southern Hemisphere circulation and relations with sea ice and sea surface temperature, J. Climate, 15: 3058-3068.

Robertson F. R., Smith P. J. 1983. The impact of model moist processes on the energetics of extratropical cyclones. Mon. Wea. Rev., 111: 723-743.

Serreze M.C. et al. 1997. Icelandic Low cyclone activity: Climatological features, linkages with the NAO, and relationships with Recent Changes in the Northern Hemisphere Circulation, J. Climate, 10: 453-464.

Simmonds I., Keay K., Lim E. 2003. Synoptic activity in the seas around Antarctica. Mon. Wea. Rev., 131: 272-288

Simmonds I., Burke C., Keay K. 2008. Arctic climate change as manifest in cyclone behavior, J. Climate, 21: 5777-5796, doi: 10.1175/2008JCLI2366.1.

Smith P. J. 1980. The energetics of extratropical cyclones. Reviews of Geophysics and Space Physics, 18: 378-386.

Stroeve J., Holland M.M., Meier W., Scambos T., Serreze M. 2007. Artic ice decline: Faster than forecast. Geophysical Research Letters, 34: L09501, doi: 10.1029/2007GL029703

Uotila J. P., Pezza A.B., Cassano J.J., Keay K., Lynch A. 2009. A comparison of low pressure system statistics derived from a high resolution NWP output and three reanalysis products over the Southern Ocean. J. Geophys. Res., 114: D17105, doi: 10.1029/2008JD01 1583. 
Veiga J.A.P., Pezza A.B., Simmonds I. Silva Dias P. L. 2008. An analysis of the environmental energetics associated with the transition of the first South Atlantic hurricane. Geophys. Res. Lett., 35: L15806, doi:10.1029/2008GL034511.

Wahab M. A., Basset, H. A., Lasheen A. M. 2002. On the mechanism of wintercyclogenesis in relation to vertical axis tilt. Meteorol. Atmos. Phys., 81: 103127. 


\section{Appendix D}

\section{Appendix D - ECHAM5 Usage Justification}

\section{D.1 Why ECHAM5?}

A major focus for the Sea of Okhotsk (SOK) paper (Chapter 5) was the extent to which sea-ice anomaly persistence affects the North Atlantic Oscillation (NAO) and storms in the North Pacific. "Persistence" in this context is defined to mean a seaice anomaly of similar sign and magnitude that shows up repeatedly in consecutive months over the course of a given season. To conduct this work it was necessary to use an atmosphere model to create an idealized study comprised of a series of scenarios ranging from a fully ice covered SOK to an ice-free SOK. To satisfactorily address these project requirements the atmospheric model had to possess the following criteria:

1) it should be a global model at a cost-efficient resolution with a demonstrated research record for storm track applications

2) it should have been used with the tracking algorithm by Hodges

3) it should represent storm tracks (and their lifecycle) realistically

4) it should have been used to study sea-ice changes

The ECHAM5 model was identified as satisfactorily addressing these requirements. 
The first point is very important in order to have a reasonable computational time and storage space for the runs since the study focus region is the entire Northern Hemisphere. An important point to emphasize is that the objective of the dissertation work was to study extra-tropical storms and not mesoscale systems (smaller storms). Mesoscale systems require higher resolution, but for that type of storms, Regional Climate Models are more suitable. Bengtsson et al. (2006) ('BHR06' hereafter) pointed out that extra-tropical synoptic-scale storms are well resolved and simulated by the ECHAM5.

The second criterion is satisfied because BHR06 used the tracking algorithm by Hodges and the ECHAM5 model. The authors concluded that ECHAM5 produces results that agree well with analyses from the 40-yr ECMWF Re-Analysis (ERA-40; Uppala et al. 2005), including the distribution of storms as a function of maximum intensity. Their storm statistics were gathered via identification and tracking, from genesis to lysis, of each individual storm in a Lagrangian framework - the same as the approach we used in the SOK paper.

The third criterion is very important. This criterion was tested by BHR06 who concluded that the ECHAM5 model provided them with "confidence that the ECHAM5 model can produce a good representation of extratropical storms". They added that there was also good agreement between the AMIP ( "Atmospheric Model Intercomparison Project" as described in Gates et al. (1999)), the ECHAM5 integration, and ERA-40.

As for the fourth criteria, the ECHAM5 model has been used to study sea-ice, as in Seierstad and Bader (2008). It is also important to emphasize that one of authors of that paper, Dr. Juergen Bader, is one of the co-authors of our Sea of Okhotsk 
paper. The sea-ice setup used by Seierstad and Bader (2008) is thus similar to our setup.

The ECHAM5 model was thus selected for the Sea of Okhotsk paper. It is supported by peer-reviewed research results that attest to its utility for this type of work, as indicted in papers by BHR06 and Seierstad and Bader (2008). It was therefore felt that the ECHAM5 model was a logical and satisfactory choice for this project.

\section{D.2 Global Climate Model Performance over Alaska, Greenland and the Extra- Tropics}

The ECHAM5 is not new to the University of Alaska Fairbanks (UAF) and the International Arctic Research Center (IARC). Walsh et al. (2008), from the UAF/IARC group, analyzed the performance of a set of 15 global climate models (including the ECHAM5) for Alaska, Greenland, the pan-Arctic and the Northern Hemisphere extra-tropical domains. One objective of their study was to understand how the different models perform for the regions aforementioned. The 15 models evaluated came from different countries and possessed a typical array of spatial resolutions and physical process handling. The highest resolution models they compared were the National Center for Atmospheric Research (NCAR) Community Climate System Model, version 3.0 (CCSM3.0) at an atmospheric resolution of T85L26, the MPI ECHAM5 model at a resolution of T63L31 and the Commonwealth Scientific and Industrial Research Organisation Mark version 3.0 (CSIRO Mk3.0) at a resolution of T63L18. The other models have lower resolutions, for example, the Canadian Centre for Climate Modelling and Analysis (CCCma) Coupled General 
Circulation Model, version 3.1 (CGCM3.1) at a resolution of T47L31 or the NCAR Parallel Climate Model, version 1 (PCM1) at a resolution of T42L18.

Walsh et al. (2008) ranked the models based on the root-mean-square error (RMSE) and other criteria in order to "identify the models that are most successful at simulating the seasonal cycle of the climates of Alaska and Greenland." The authors concluded that the top-performing models for Alaska are the Geophysical Fluid Dynamics Laboratory Climate Model, version 2.1 (GFDL CM2.1, atmospheric resolution of $\left.2.5^{\circ} \times 2.5^{\circ} \mathrm{L} 24\right)$, the MPI ECHAM5, the Centre National de Recherches Météorologiques Coupled Global Climate Model, version 3 (CNRM-CM3, atmospheric resolution of T42L45), the Met Office (UKMO) third climate configuration of the Unified Model (HADCM3, atmospheric resolution of $3.75^{\circ} \mathrm{x}$ $2.5^{\circ} \mathrm{L}$ ), and the Model for Interdisciplinary Research on Climate 3.2 (MIROC3.2, atmospheric resolution of T42L20). For Greenland, the top-performing models were the GFDL CM2.1, the MIROC3, the CNRM-CM3 and the MPI ECHAM5.

For the larger domains, which encompassed a pan-Arctic region $\left(60^{\circ}-90^{\circ} \mathrm{N}\right.$ and $20^{\circ}-90^{\circ} \mathrm{N}$ ), the two highest-ranking models are the MPI ECHAM5 and GFDL CM2.1 (Walsh et al. 2008). The authors also mention that "there is strong overlap among the top performers for the larger domains (pan-Arctic and Northern Hemisphere) and the subregional domains (Alaska and Greenland)...MPI ECHAM5 and GFDL CM2.1 are clearly the highest-ranking models overall". Walsh et al. (2008) also consider these models “...the logical candidates for driving offline simulations of high-latitude variables such as permafrost, glaciers/ice sheets, and terrestrial or marine ecosystems." 
When it comes to the resolution, it is surprising that the higher resolution model (CCSM3.0) in their 15-model comparison did not rank among the higherranking models. Walsh et al. (2008) comment that there was no systematic relationship to the model resolution and they add that the models with the smallest RMSEs (MPI ECHAM5 and GFDL CM 2.1) "have resolutions that are neither the highest nor lowest of the 15 models." And they also mention that there is no obvious relationship between model performance and the type of sea ice formulation in the models.

Hence, the paper by Walsh et al. (2008) from the UAF/IARC group is another strong reason for the use of the ECHAM5 model when studying the Alaska region and extra-tropical areas as well.

\section{D.3 ECHAM5 and the Hodges Algorithm}

BHR06 emphasized in their paper that any systematic change in the intensity, frequency, or position of storm tracks would have a far-reaching influence on the local climate. Therefore, for their study, it was very important to make a selection of a climate model that would represent the extra-tropical storms accurately. BHR06 also points out that a number of numerical simulations with GCMs at sufficient resolution have shown considerable skill in reproducing these systems (Bengtsson 1991), and that several studies had been performed for climate change scenarios and for particular models: Carnell et al. $(1996 ; 2.5 \times 3.75$, L11), who used the United Kingdom Meteorological Office (UKMO) coupled ocean-atmosphere model at a horizontal resolution of $2.5^{\circ} \times 3.75^{\circ}$ latitude/longitude with 11 vertical levels in the atmosphere and 17 levels in the ocean; Schubert et al. (1998; T42L19), who used the 
ECHAM3 model at a resolution of T42L19; Sinclair and Watterson (1999), who used the Commonwealth Scientific and Industrial Research Organisation nine-level GCM (CSIRO9 GCM) at a resolution of R21L9; Leckebusch and Ulbrich (2004), who used the Hadley Centre global general circulation model (HadCM3) at a resolution of $2.5^{\circ}$ x 3.75 L19; and Geng and Sugi (2003), who used the Japan Meteorological Agency (JMA) forecast model (GSM8911) at a resolution of T106L21.

However, these studies were constrained to using as the basis for storm identification the MSLP field or geostrophic vorticity as derived from MSLP. As well, the data used in most of these studies have been derived from relatively low resolution integrations of the respective GCMs as indicated by the information (atmosphere only), with the exception of Geng and Sugi (2003) who used higherresolution data. Several other studies, as pointed out in BHR06, identified extratropical cyclones using MSLP but did not track them (Lambert 1995; Zhang and Wang 1997; Fyfe 2003).

In the ECHAM5 model (Roeckner et al. 2003) vorticity, divergence, temperature, and the logarithm of surface pressure are represented by a truncated series of spherical harmonics with triangular truncation $\mathrm{T} 63$, whereas the advection of water vapor, cloud liquid water, and cloud ice is treated by a flux-form semiLagrangian scheme. A hybrid sigma/pressure system is used in the vertical direction (31 layers with the top model level at $10 \mathrm{hPa}$ ). The model uses state-of-the-art parameterizations for shortwave and longwave radiation, stratiform clouds, cumulus convection, boundary layer and land surface processes, and gravity wave drag. This is the same configuration used in the SOK paper, that is, using a T63 resolution, which is equivalent to about 1.875 degrees $\times 1.85$ degrees. The same type of configuration 
used in BHR06 for the atmosphere part.

As described in BHR06, the relative vorticity field at moderate to high resolution can be too noisy for tracking studies. Therefore, the analysis of extratropical cyclones is performed at a spectral resolution of T42 on a Gaussian grid for all datasets. This makes it also possible to compare the results with other papers, which used the Hodges' algorithm.

BHR06 contrasted the storm tracks derived from the ERA-40 reanalysis (period: 1979-2003) with those from the three-ensemble AMIP integrations of ECHAM5 available for the period 1979-99 to explore and validate the model response to the prescribed boundary forcing. They point out that the ECHAM5 model reproduces the extratropical storm tracks in "surprisingly good agreement with the analyzed data from ERA-40". ECHAM5 is also capable of reproducing the Northern Hemisphere storm track response to the El Niño Southern Oscillation - ENSO (BHR06). Besides that, ECHAM5 also reproduces "the statistical distribution of the number of storms as a function of their maximum intensity in surprisingly good agreement with ERA-40 on hemispheric regions".

Even though one of the caveats of the paper by BHR06 is that the experiments were restricted to one single coupled model (for future climate change studies), they add that the atmospheric version of the model is capable of reproducing essential features of the life cycle, distribution, and intensity of transient storms in the extratropics. These were all features we were looking for when selecting a model. 


\section{D.4 The Instantaneous Removal of Sea Ice and the Use of Climatological SST}

BHR06 also used three AMIP-style simulations performed for the time period 1979-99, using the atmospheric component of the coupled model. Hurrell et al. (2008) mentions that the AMIP is a collection of integrations where an AGCM is forced with the known global evolution of Sea Surface Temperatures (SST) and Sea Ice Concentrations (SIC) and that the AMIP is an example of an "experimental protocol that has become a standard...". In Chapter 5, we presented the Sea of Okhotsk paper. In one of the experiments we removed sea-ice, and in that case the AMIP II Sea Surface Temperatures (Hurrell et al. 2008) were used.

Hurrell et al. (2008) provides a description of the SST and SIC dataset used in the AMIP project. The dataset was created to provide SST and SIC boundary dataset for uncoupled simulations with present and future versions of CAM - the Community Atmosphere Model (Collins et al. 2006). The CAM is a three-dimensional atmospheric general circulation model, which serves as the atmospheric component of the CCSM model (Community Climate System Model). The Hurrell et al. (2008) dataset is used in the AMIP project as well.

The dataset by Hurrell et al. (2008) is a merged product based on the monthly mean Hadley Centre sea ice and SST version 1 (HadISST1) and the National Oceanic and Atmospheric Administration (NOAA) weekly optimum interpolation (OI) SST analysis version 2 . These datasets were also used to provide ocean surface information to the 40-year European Centre for Medium-Range Weather Forecasts Re-Analysis (ERA-40).

In our "no-ice" experiment in the Sea of Okhotsk paper, we used the climatological SST data from the AMIP II project, and as described before, it is a 
state-of-the-art dataset. The removal of sea-ice and the use of a climatological SST is not a new procedure and has seen use in previous experiments; for example, Schröder and Connolley (2007) using the HadCM3 climate model from the Hadley Centre, removed sea-ice in their experiments. The authors mentioned that they did not modify the temperature of the mixed-layer temperature in some of their experiments, which was close to freezing point during winter.

Magnusdottir et al. (2004) and Deser et al. (2004) used the Community Climate Model (CCM3) to study changes in the atmospheric circulation based on SST and sea ice anomalies. The resolution used was T42L18, lower than the resolution we used in the Sea of Okhotsk paper (Chapter 5). Magnusdottir et al. (2004) describes that: "We use two different boundary forcings, that of SST anomalies and that of sea ice anomalies. When forcing the model with SST anomalies, we hold the sea ice extent constant at climatological values. When forcing with sea ice anomalies, we hold the SST distribution constant at climatological values." In Deser et al. (2007), the CCM3 at T42L18 was used for similar experiments and climatological SSTs were also used when considering the sea-ice anomalies. In Bhatt et al. (2007), the Community Climate Model CCM version 3.6 was used at a resolution of T42L18 with climatological SSTs as well. In Deser et al. (2009), the Community Atmospheric Model CAM3 model was used at resolutions of T85, the climatological SSTs were used in the sea-ice experiment to "isolate the impact of the sea ice changes". Hence, the idea of using climatological values for SST when forcing the model with sea ice anomalies is not new and tried with the CCM3 model. 
Honda et al. (1999) studied the changes in sea-ice in the Sea of Okhotsk, assuming different sea-ice settings ( $0 \%$ or $100 \%$ sea-ice concentration) from December to February. They used an AGCM developed at the Meteorological Research Institute of the Japan Meteorological Agency at a resolution of T21L30 much lower than our Sea of Okhotsk study (Chapter 5). And the authors add that the "distribution of sea surface temperature (SST) and sea-ice cover in the model were prescribed as their climatological means based on the observed monthly mean data."

Hence, the objective of the modeling section of our Sea of Okhotsk paper was to test the idea of persistence from the extremes of total sea-ice cover and no sea-ice in the Sea of Okhotsk. The "no-ice" part was based on an instantaneous removal of sea-ice. The SST used was a climatology from the AMIP II project - and our experiments showed two completely different sea-ice conditions: $100 \%$ sea-ice concentration over a large area in the Sea of Okhotsk and no-sea ice - these two extremes alone provided enough "perturbation" in the atmosphere. The instantaneous removal of sea-ice provided enough fluxes to the atmosphere (Fig. 5.10 in Chapter 5), compared with the $100 \%$ experiment in which the sea-ice acted as a "lid" to the fluxes. Furthermore, the use of climatological SSTs when sea-ice is removed is not new, as mentioned before, and our Sea of Okhotsk study presents a fresh perspective on that kind of analysis at comparatively high spatial resolution for this category of experiment, e.g. as compared with Magnusdottir et al. (2004), Deser et al. (2004) and Honda et al. (1999). 


\section{D.5 ECHAM5 Description}

Following is a description of the Max Planck Institute for Meteorology model ECHAM5 along with an assessment of horizontal and vertical resolution sensitivity.

\section{D.5.1 ECHAM5 General Description}

The Max Planck Institute for Meteorology report number 349 (Roeckner et al., 2003) provides a thorough description of the ECHAM5 model. Here, a summary is provided.

The ECHAM5 was developed at the Max Planck Institute for Meteorology (MPIM); 5.0 is the most recent version. The different ECHAM model versions come originally from the spectral weather prediction model of the European Centre for Medium Range Weather Forecasts (ECMWF). Hence, there is a connection between the MPIM and the ECMWF embedded in ECHAM5.

The dynamical part of the ECHAM5 is formulated in spherical harmonics truncated expansions in terms of spherical harmonics are used for the representation of the dynamical fields. Non-linear terms are evaluated at a set of almost regularly distributed grid points (Gaussian grid). A flexible vertical coordinate is used, so that the model can use either the terrain-following sigma coordinate (Phillips 1957) or a hybrid coordinate: the sigma system at the lowest model level gradually transforms into a pressure system in the lower stratosphere (Simmons and Burridge 1981), which is computationally efficient. The highest computational level is at $10 \mathrm{hPa}$ with a total of 19 or 31 levels in the standard configuration. The derivations of the governing equations (used in ECHAM5) into a spectral representation are also presented in Roeckner et al. (2003). 
The semi-implicit leapfrog time differencing scheme developed by Robert et al. (1972) is used for the divergence, temperature and surface pressure equations. The growth of spurious computational modes is inhibited by the time filter of Asselin (1972). A semi-implicit method is also used for the zonal advection terms in the vorticity equation following Robert $(1981,1982)$.

The horizontal diffusion is based on a numerically convenient form of the scale selective diffusion with coefficients determined empirically to ensure a realistic behavior of the resolved scales. The turbulent flux of a variable at the surface is given by a bulk transfer relation. This relation is connected to a transfer coefficient obtained from Monin-Obukhov similarity theory by integrating the flux-profile relationships over the lowest model layer.

As for the surface processes, the implicit coupling scheme by Schulz et al. (2001) is used which is unconditionally stable and allows the synchronous calculation of the prognostic variables and the surface fluxes. The water budget equations are given for: snow intercepted by the canopy, snow at the surface, rain water intercepted by the canopy and soil water. A simple scheme is used for calculating the water temperature, ice thickness and ice temperature of lakes.

The sea-ice concentration is interpolated in time from monthly observations while sea-ice thickness is prescribed at $2 \mathrm{~m}$ for the Northern Hemisphere, if the seaice fraction is larger than 0.01 , otherwise, the sea-ice fraction and thickness is zero. The sea-ice temperature is calculated similarly to the lake temperature, with the formulation given in Roeckner et al. (2003). The freezing temperature of saline water is set to $-1.77^{\circ} \mathrm{C}$. For most surfaces, the albedo of snow and ice is assumed to be a linear function of surface temperature. Over water surfaces, the albedo is set to a 
constant value of 0.07 and when a grid-cell is partially covered with sea ice, the gridmean albedo is given as a weighted average between the fractional areas of sea and ice. Over snow-free land surfaces, and annual mean background albedo, derived from satellite data is used (Hagemann 2002).

The ECHAM5 model uses the Subgrid Scale Orographic Parameterization (SSOP) (Lott and Miller 1997) to represent the effects of orographic variations on scales smaller than the typical horizontal resolution of a climate model. This methodology takes into account the interaction between the orography and the atmospheric flow through two mechanisms: momentum transfer from the earth to the atmosphere through the orographic gravity waves and the drag exerted by the subgrid scale mountains when the air flow is blocked at low levels.

The mass flux scheme of Tiedtke (1989) is applied for cumulus convection. An ensemble of clouds consisting of updrafts and downdrafts in a steady state represents the contribution of cumulus convection to the large-scale budgets of heat, moisture and momentum. The stratiform clouds (vapor, liquid, and ice phase) are given by the cloud microphysical scheme of Lohmann and Roeckner (1996) and a statistical cloud cover scheme including prognostic equations for the distribution moments by Tompkins (2002).

Because of the complexity of the radiative transfer calculations in a model, some approximations and simplifications are inevitable. Thus the radiative transfer computation is called less frequently than the dynamics and other parameterizations. The radiation time step is thus set to 2 hours. At each 2 -hour timestep, the transfer calculation is executed at all grid points of the Gaussian grid.

As for the atmospheric composition: water vapor and cloud water and ice are 
prognostic variables, whereas cloud cover is diagnostic; ozone is prescribed based on the climatology of Fortuin and Kelder (1998); carbon dioxide, methane, N2O, CFCs are prescribed with constant mixing ratios; aerosol distributions are prescribed based on Tanre et al. (1984).

In ECHAM5, the solar radiation at each timestep depends on the annular mean solar irradiation, the relative variation of the actual sun-Earth distance, and the cosine of the local solar zenith angle. The shortwave radiative transfer is based on Fouquart and Bonnel (1980), which include Rayleigh scattering, absorption of water vapor and ozone. The scheme has four spectral bands, one for the visible and UV range, and three for the near infrared. Aerosols and cloud particles are effective by absorption and scattering. The longwave transfer parameterization follows the scheme of Mlawer et al. (1997) called "Rapid Radiative Transfer Model" (RRTM). A total of 16 spectral bands are used, including line absorption by $\mathrm{H}_{2} \mathrm{O}, \mathrm{CO}_{2}, \mathrm{O}_{3}, \mathrm{CH}_{4}$, $\mathrm{N}_{2} \mathrm{O}, \mathrm{CFC}-11, \mathrm{CFC}-12$ and aerosols.

ECHAM5 also takes into account the Earth's orbital variations on radiation, by using two orbital parameters derived from two groups: the VSOP (Variations Séculaires des Orbites Planétaires) analytical solution by Bretagnon and Francou (1988), which gives precise orbit determination principles to reflect short term variations for today's climate; and an orbit that uses Keplerian laws only, allowing for paleoclimate studies adjustment using the long term series expansions for obliquity, eccentricity, and precession by Laskar and Boudin (1993). The solution for the position of the Earth is based on heliocentric spherical coordinate variables and the reference frame is the mean equinox and ecliptic of date. 


\section{D.5.2 ECHAM5 Sensitivity to Horizontal and Vertical Resolution}

The Max-Planck Institute for Meteorology report number 354 (Roeckner et al., 2004) describes the impact of changes in horizontal and vertical resolution on seasonal mean climate. A summary of the report is provided here. A series of experiments using resolutions that ranged from T21L19 to T159L31 was performed. Briefly, they showed that an increase in horizontal resolution led to a warming of the troposphere at mid-latitudes, and thus a poleward shift and intensification of the midlatitude westerlies. An increase in the vertical resolution alone had the opposite effect (almost independent of the horizontal resolution): the atmosphere is colder at low and middle latitudes, and warmer at high latitudes. In addition to temperature bias, the authors also observed a decrease and equatorward shift of the mid-latitude westerlies.

Roeckner et al. (2004) mention that several studies confirm the reduction of errors in medium-resolution models (e.g.: T42, T63) when compared to lowresolution ones (e.g.: T21). This conclusion applies not only to seasonal mean climate but also to transient features like eddy fluxes, extra-tropical cyclones or blocking events. This comment is very important for the kind of work outlined in Chapter 5 of the dissertation, since the primary focus is extra-tropical cyclones. For higher resolutions (> T63), Roeckner et al. (2004) says that the conclusions differ between models and also depend on the climate statistics investigated. This emphasizes the fact that one needs to be careful since an increase in the model resolution does not mean that better results will be obtained and an increase in horizontal resolution should also follow an increase in vertical resolution - which can be computationally expensive.

In many of the papers outlined in Roeckner et al. (2004), increasing the 
resolution from $\mathrm{T} 42$ to $\mathrm{T} 106$ increased tropospheric temperatures, predominantly at mid-latitudes, resulting in a poleward shift and intensification of the westerly jets (for example, in Boyle 1993, Déqué et al. 1994 and Stendel and Roeckner 1998). The reason for the warming was due to the latent heat release due to an intensification of the hydrological cycle, as outlined in Pope and Stratton (2002). The poleward shift and intensification of the westerlies led to increased systematic errors in high resolution experiments. The systematic errors also point to the fact that the vertical and horizontal resolution should be chosen in a consistent manner to avoid the misrepresentation of horizontal and vertical scales, according to the quasi-geostrophic theory by Lindzen and Fox-Rabinovitz (1989). Roeckner et al. (2004) emphasize that higher resolution is particularly important in the tropics, for example, when studying tropical cyclones, and high vertical resolution seems to be a necessary condition for studying other processes in the tropics such as the Madden-Julian Oscillation.

In order to assess the resolution sensitivity in ECHAM5, a series of AMIPstyle experiments (Gates et al., 1999) were conducted using observed monthly sea surface temperatures and sea-ice cover for the period 1978-1999. Their experiments differed only with respect to the horizontal and vertical resolutions - from T21L19 to T159L31.

As for the L19 experiments, there was little evidence for convergence to a more realistic climate state at horizontal resolutions higher than T42. In the L31 cases, the root-mean-squared error decreased monotonously with increasing horizontal resolution - from T42 to T159. Thus, the L31 are superior to the L19 experiments. Roeckner et al. (2004) also emphasize that the L19 vertical resolution is adequate for T31 and T42, whereas the L31 is adequate for T63 and T85. Similarly, 
higher vertical resolutions would also be required for T106 and T159.

It is important to note that Roeckner et al. (2004) recognize that a more balanced choice of horizontal/vertical resolutions does not only improve the performance of the model, but it is beneficial from an economic point-of-view (costs of the computing hours). They give an example of a comparison between the T106L19 and the T63L31 experiments. The T63L31 is more efficient in terms of CPU time (about $65 \%$ of the T106L19 experiment) and it is also more accurate by more than $10 \%$ in terms of the overall root-mean-square error! They also add that a further gain of $10 \%$ can only be achieved at very high cost (factor of 13 ) for a run at T159L31. But, as they point out, “...the choice of resolution would be more subjective because the lack of accuracy in T63L 31 can be balanced by the advantages of longer simulation lengths and larger ensembles." - that is, greater statistical stability in the final results.

Hence, the selection of the ECHAM5 model as the basis of the sea-ice anomaly persistence experiment in the Sea of Okhotsk paper (Chapter 5 of the dissertation) at a resolution of $\mathrm{T} 63 \mathrm{~L} 31$ is satisfactory from several perspectives -it is more efficient computationally and it produces an accurate result well suited to this work, a result echoed by various researchers including Walsh et al. (2008), who used the same model at the same resolution. 


\section{References}

Asselin, R., 1972: Frequency filter for time integrations. Mon. Wea. Rev., 100, 487490.

Bengtsson, L., 1991: Advances and prospects in numerical weather prediction. Quart. J. Roy. Meteor. Soc., 117, 855-902.

Bengtsson, L., K.I. Hodges, and E. Roeckner, 2006: Storm tracks and climate change. J. Clim., 19, 3518-3543.

Bhatt, U. S., M. A. Alexander, C. Deser, J. E. Walsh, J. S. Miller, M. Timlin, J. D. Scott, and R. Tomas, 2008: The atmospheric response to realistic reduced summer Arctic sea ice anomalies. Arctic Sea Ice Decline: Observations, Projections, Mechanisms, and Implications, Geophys. Monogr. Ser., 180, E. T. DeWeaver, C. M. Bitz, and L. -B. Tremblay, Eds., AGU, 91-110.

Boyle, J. S., 1993: Sensitivity of dynamical quantities to horizontal resolution for a climate simulation using the ECMWF (cycle 33) model. J. Climate, 6, 796-815.

Bretagnon, P. and G. Francou, 1988: Planetary theories in rectangular and spherical variables - VSOP 87 solutions. Astronomy and Astrophysics, 202, 309-315.

Carnell, R.E., C. A. Senior, and J. F. B. Mitchell, 1996: An assessment of measures of storminess: simulated changes in northern hemisphere winter due to increasing CO2. Climate Dyn., 12, 467-476.

Collins, W. D. and Coauthors, 2006: The formulation and atmospheric simulation of the Community Atmosphere Model Version 3 (CAM3). J. Climate, 19, 2144-2161.

Déqué, M., C. Dreverton, A. Braun, and D. Cariolle, 1994: The ARPEGE/IFS atmosphere model: a contribution to the French community climate modeling. Clim. Dyn., 10, 249-266.

Deser, C., R. Tomas, M. Alexander, and D. Lawrence, 2009: The seasonal atmospheric response to projected Arctic sea ice loss in the late 21 st century. $J$. Climate, in press. 
Deser, C., R. A. Tomas, and S. Peng, 2007: The transient atmospheric circulation response to North Atlantic SST and sea ice anomalies. J. Climate, 20, 4751-4767

Deser, C., G. Magnusdottir, R. Saravanan, and A. Phillips, 2004: The Effects of North Atlantic SST and Sea Ice Anomalies on the Winter Circulation in CCM3. Part II: Direct and Indirect Components of the Response. J. Climate, 17, 877-889.

Fortuin, J. P. F. and H. Kelder, 1998: An ozone climatology based on ozonesonde and satellite measurements. J. Geophys. Res., 103, 31709-31734.

Fouquart, Y. and B., Bonnel, 1980: Computations of solar heating of the earth's atmosphere: A new parameterization. Beitr. Phys. Atmos., 53, 35-62.

Fyfe, J. C., 2003: Extratropical Southern Hemisphere cyclones: harbingers of climate change. J. Climate, 16, 2802-2805.

Gates, W. L. and Coauthors, 1999: An overview of the results of the Atmospheric Model Intercomparison Project (AMIP I). Bull. Amer. Met. Soc., 80, 29-55.

Geng, Q., and M. Sugi, 2003: Possible change of extratropical cyclone activity due to enhanced greenhouse gases and sulfate aerosols - study with a high-resolution AGCM. J. Climate, 16, 2262-2274.

Hagemann, S., 2002: An improved land surface parameter dataset for global and regional climate models. Report 336, Max-Planck-Institut für Meteorologie, Hamburg.

Honda, M., K. Yamazaki, H. Nakamura, and K. Takeuchi, 1999: Dynamic and Thermodynamic Characteristics of Atmospheric Response to Anomalous Sea-Ice Extent in the Sea of Okhotsk. J. Climate, 12, 3347-3358.

Hurrell, J.W., J.J. Hack, D. Shea, J.M. Caron, and J. Rosinski, 2008: A New Sea Surface Temperature and Sea Ice Boundary Dataset for the Community Atmosphere Model. J. Climate, 21, 5145-5153

Lambert, S. J., 1995: The effect of enhanced greenhouse warming on winter cyclone frequencies and strengths. J. Climate, 8, 1447-1452.

Laskar, J. and F. Boudin, 1993: Orbital precessional, and insolation quantities for the 
earth from -20 myr to +10 myr. Astronomy and Astrophysics, 270, 522-533.

Leckebusch, G. C., and U. Ulbrich, 2004: On the relationship between cyclones and extreme windstorm events over Europe under climate change. Global Planet. Change, 44, 181-193.

Lindzen, R. S. and M. S. Fox-Rabinovitz, 1989: Consistent vertical and horizontal resolution. Mon. Wea. Rev., 117, 2575-2583.

Lohmann, U. and E. Roeckner, 1996: Design and performance of a new cloud microphysics scheme developed for the ECHAM4 general circulation model. Clim. Dyn., 12, 557-572.

Lott, F. and M. J. Miller, 1997: A new-subgrid-scale orographic drag parameterization: Its formulation and testing. Quart. J. Roy. Meteor. Soc., 123, 101127.

Magnusdottir, G., C. Deser, and R. Saravanan, 2004: The Effects of North Atlantic SST and Sea Ice Anomalies on the Winter Circulation in CCM3. Part I: Main Features and Storm Track Characteristics of the Response. J. Climate, 17, 857-876.

Mlawer, E. J., and Coauthors, 1997: Radiative transfer for inhomogeneous atmospheres: RRTM, a validated correlated-k model for the longwave. J. Geophys. Res., 102, 16663-16682.

Phillips, N. A., 1957: A coordinate system having some special advantages for numerical forecasting. Journal of Meteorology, 14, 184-185.

Pope, V. D. and R. A. Stratton, 2002: The processes governing horizontal resolution sensitivity in a climate model. Clim. Dyn., 19, 211-236.

Robert, A. J., 1981: A stable numerical integration scheme for the primitive meteorological equations. Atmos. Ocean, 19, 35-46.

Robert, A. J., 1982: A semi-Lagrangian and semi-implicit numerical integration scheme for the primitive meteorological equations. J. Met. Soc. Japan, 60, 319-325.

Robert, A. J., J. Henderson and C. Turnbull, 1972: An implicit time integration scheme for baroclinic models in the atmosphere. Mon. Wea. Rev., 100, 329-335. 
Roeckner, E., and Coauthors, 2003: The atmospheric general circulation model ECHAM5. Part I: Model description. MPI Rep. 349, 127 pp.

Roeckner, E., and Coauthors, 2004: The atmospheric general circulation model ECHAM5. Part II: Sensitivity of simulated climate to horizontal and vertical resoution. MPI Rep. 354, 55 pp.

Schröder, D. and W. M. Connolley, 2007: Impact of instantaneous sea ice removal in a coupled general circulation model. Geophys. Res. Lett., 34, L14502, doi: $10.1029 / 2007$ GL030253.

Schubert, M., J. Perlwitz, R. Blender, K. Fraedrich, and F. Lunkeit, 1998: North Atlantic cyclones in $\mathrm{CO} 2$ induced warm climate simulations: frequency, intensity, and tracks. Climate Dyn., 14, 827-837.

Schulz, J.-P., L. Dümenil, and J. Polcher, 2001: On the land surface-atmosphere coupling and its impact in a single-column atmospheric model. J. Appl. Meteorol., 40, 642-663.

Seierstad, I. A. and J. Bader, 2008: Impact of a projected future Arctic sea ice reduction on extratropical storminess and the NAO. Clim. Dyn., doi: 10.1007/s00382008-0463-x.

Simmons, A. J. and D. M. Burridge, 1981: An energy and angular-momentum conserving vertical finite difference scheme and hybrid vertical coordinates. Mon. Wea. Rev., 109, 758-766.

Sinclair, M. R., and I. G. Watterson, 1999: Objective assessment of extratropical weather systems in simulated climates. J. Climate, 12, 3467-3485.

Stendel, M. and E. Roeckner, 1998: Impacts of horizontal resolution on simulated climate statistics in ECHAM4. MPI Rep. 253, 57 pp.

Tanre, D., J.-F. Geleyn and J. M. Slingo, 1984: First results of the introduction of an advanced aerosol-radiation interaction in the ECMWF low resolution global model. In: Aerosols and Their Climatic Effects, edited by Gerber H. and A. Deepak, pp. 133177. A. Deepak, Hampton, Va.

Tiedtke, M., 1989: A comprehensive mass flux scheme for cumulus parameterization in large-scale models. Mon. Wea. Rev., 117, 1779-1800. 
Tompkins, A., 2002: A prognostic parameterization for the subgrid-scale variability of water vapor and clouds in large-scale models and its use to diagnose cloud cover. J. Atmos. Sci., 59, 1917-1942.

Uppala, S. M., and Coauthors, 2005: The ERA40 reanalysis. Quart. J. Roy. Meteor. Soc., 131, 2961-3012.

Walsh, J. E., W. L. Chapman, V. Romanovsky, J. H. Christensen and M. Stendel, 2008: Global climate model performance over Alaska and Greenland. J. Climate, 21, 6156-6174.

Zhang Y., and W.-C. Wang, 1997: Model-simulated northern winter cyclone and anticyclone activity under a greenhouse warming scenario. J. Climate, 10, 1616-1634. 